\title{
Uncertainty Quantification of Calculated Temperatures for the AGR-1 Experiment
}

The INL is a

U.S. Department of Energy

National Laboratory

operated by

Battelle Energy Alliance

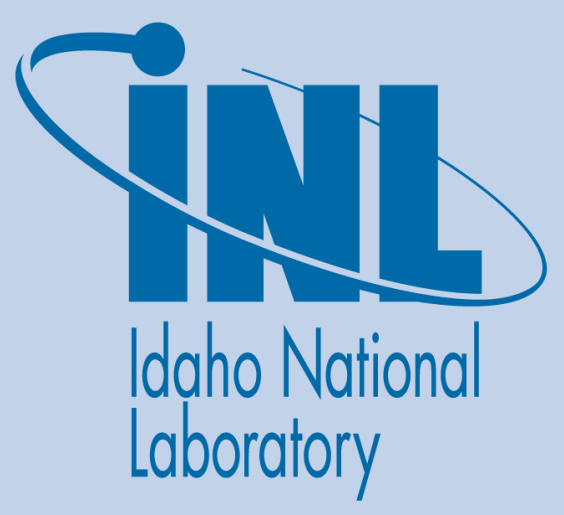

Binh T. Pham Jeffrey J. Einerson Grant L. Hawkes

March 2013

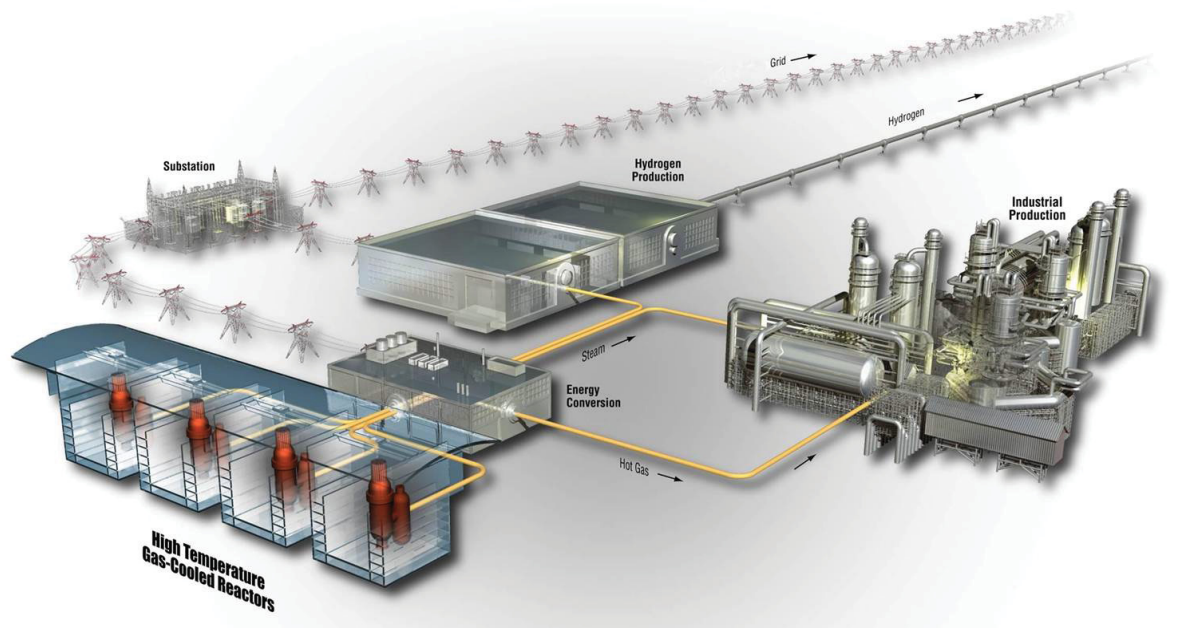




\section{DISCLAIMER}

This information was prepared as an account of work sponsored by an agency of the U.S. Government. Neither the U.S. Government nor any agency thereof, nor any of their employees, makes any warranty, expressed or implied, or assumes any legal liability or responsibility for the accuracy, completeness, or usefulness, of any information, apparatus, product, or process disclosed, or represents that its use would not infringe privately owned rights. References herein to any specific commercial product, process, or service by trade name, trade mark, manufacturer, or otherwise, does not necessarily constitute or imply its endorsement, recommendation, or favoring by the U.S. Government or any agency thereof. The views and opinions of authors expressed herein do not necessarily state or reflect those of the U.S. Government or any agency thereof. 
INL/EXT-12-25169

Revision 1

\title{
Uncertainty Quantification of Calculated Temperatures for the AGR-1 Experiment
}

\author{
Binh T. Pham \\ Jeffrey J. Einerson \\ Grant L. Hawkes
}

March 2013

\begin{abstract}
Idaho National Laboratory
VHTR Program

Idaho Falls, Idaho 83415
\end{abstract}

http://www.inl.gov

Prepared for the

U.S. Department of Energy

Office of Nuclear Energy

Under DOE Idaho Operations Office

Contract DE-AC07-05ID14517 



\section{VHTR Program}

\section{Uncertainty Quantification of Calculated Temperatures for the AGR-1 Experiment}

INL/EXT-12-25169

Revision 1

Approved by:

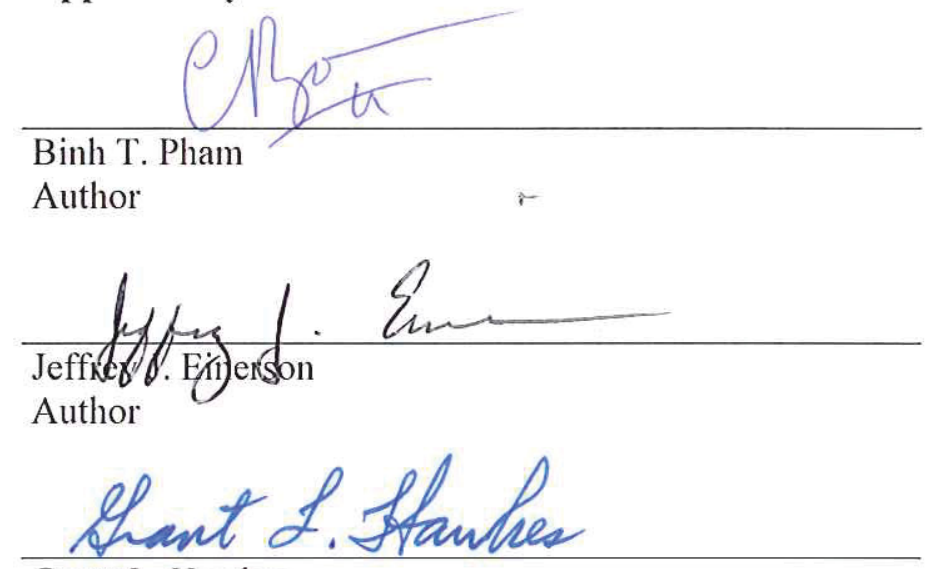

Grant L. Hawkes

Author

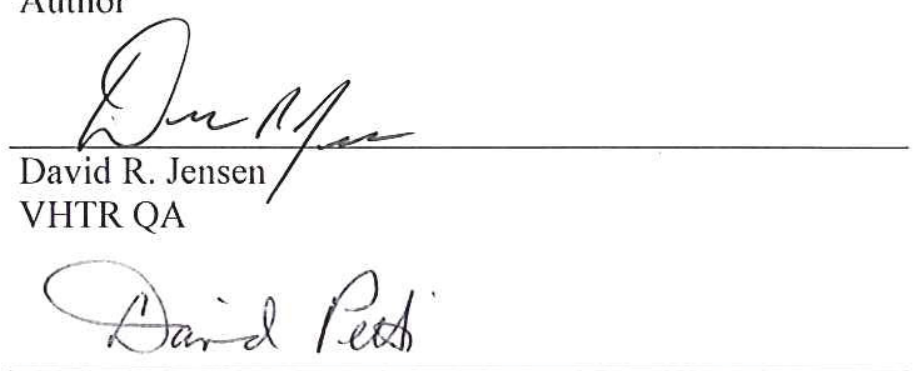

David A. Petti $\frac{3 / 11 / 2013}{\text { Date }}$

VHTR TDO Director
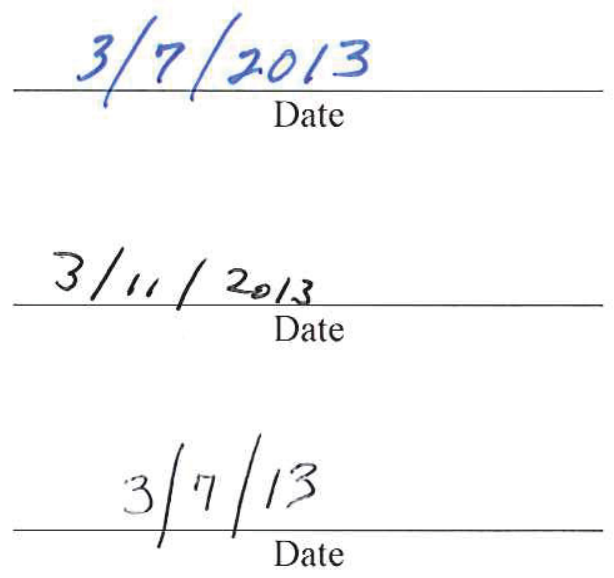



\begin{abstract}
This report documents the quantification of uncertainty of the calculated temperature data for the first Advanced Gas Reactor (AGR-1) fuel irradiation experiment conducted in the Advanced Test Reactor (ATR) at Idaho National Laboratory in support of the Very High Temperature Reactor (VHTR) Research and Development (R\&D) program. Recognizing uncertainties inherent in physics and thermal simulations of the AGR-1 test, the results of the numerical simulations are used in combination with statistical analysis methods to improve qualification of measured data. The temperature simulation data for AGR tests are also used for validation of the fission product transport and fuel performance simulation models. These crucial roles of the calculated fuel temperatures in ensuring achievement of the AGR experimental program objectives require accurate determination of the model temperature uncertainties. To quantify the uncertainty of AGR calculated temperatures, this study identifies and analyzes ABAQUS model parameters of potential importance to the AGR-1 predicted fuel temperatures. Expert judgment is used as the basis to specify the uncertainty range for a set of select parameters, including those with high sensitivity and those with large uncertainty. Propagation of model parameter uncertainty and sensitivity is then used to quantify the overall uncertainty of AGR-1 calculated temperatures.

The sensitivity analysis performed in this work went beyond the traditional local sensitivity. Using experimental design, analysis of pairwise interactions of model parameters was performed to establish sufficiency of the first-order (linear) expansion terms in constructing the response surface. To achieve completeness, uncertainty propagation made use of pairwise noise correlations of model parameters. Furthermore, using an interpolation scheme over the input parameter domain, the analysis obtains time-dependent sensitivity over the test campaign duration. This allows computation of uncertainty for the predicted peak fuel temperatures and the predicted graphite temperatures at TC locations over the whole AGR-1 irradiation period.
\end{abstract}




\section{SUMMARY}

\section{S1. Introduction}

This report documents the quantification of uncertainty in the calculated temperatures for the first Advanced Gas Reactor (AGR-1) fuel irradiation experiment. These experiments, conducted in the Advanced Test Reactor (ATR) at Idaho National Laboratory (INL), are in support of the Very High Temperature Reactor (VHTR) Research and Development (R\&D) program. While not possible to obtain by direct measurements in the tests, crucial fuel conditions (e.g., temperature, neutron fast fluence, and burnup) are calculated using core physics (JMOCUP) and thermal modeling (ABAQUS) codes. Calculated fuel temperatures serve crucial roles in achieving AGR experimental program objectives and require accurate determination of the model temperature uncertainties. One such role is the validation of the fission product transport and fuel performance simulation models.

\section{S2. Approach}

To quantify the uncertainty of AGR calculated temperatures, ABAQUS code's finite element-based thermal model input parameters of potential importance are identified. Identification has two parts: (1) using expert judgment, determine parameters with the largest uncertainties and estimate these uncertainties, and (2) using sensitivity analysis, determine parameters that the modeling is most sensitive to. A set of parameters is selected for predicted temperature uncertainty quantification, including those with high sensitivity and those with large uncertainty. The parameter uncertainties and sensitivity coefficients are combined and propagated to quantify the overall uncertainty using Eq. S1, because the predicted temperature can be assumed to be the weighted summation of input parameters. This assumption is confirmed during sensitivity analysis.

$\sigma_{T}^{2}=\sum_{i}^{n} a_{i}^{2} \sigma_{i}^{2}+\sum_{i}^{n} \sum_{j \neq i}^{n} \rho_{i j} a_{i}^{2} \sigma_{i}^{2} a_{j}^{2} \sigma_{j}^{2}$

where: $\sigma_{T}^{2}$ is the overall uncertainty in terms of variance,

$a_{i}^{2}$ is the square of the sensitivity coefficient for parameter $i$,

$\sigma_{i}^{2}$ is the uncertainty of input parameter $i$ in terms of variance,

$\rho_{i j}$ is the correlation coefficient for input parameters $i$ and $j$.

The identified-as-important model parameters in terms of influence on uncertainty are: control gas gap width, heat rate in fuel compacts, neon fraction, graphite conductivity, and fuel conductivity. These are shown in Figure S1. 


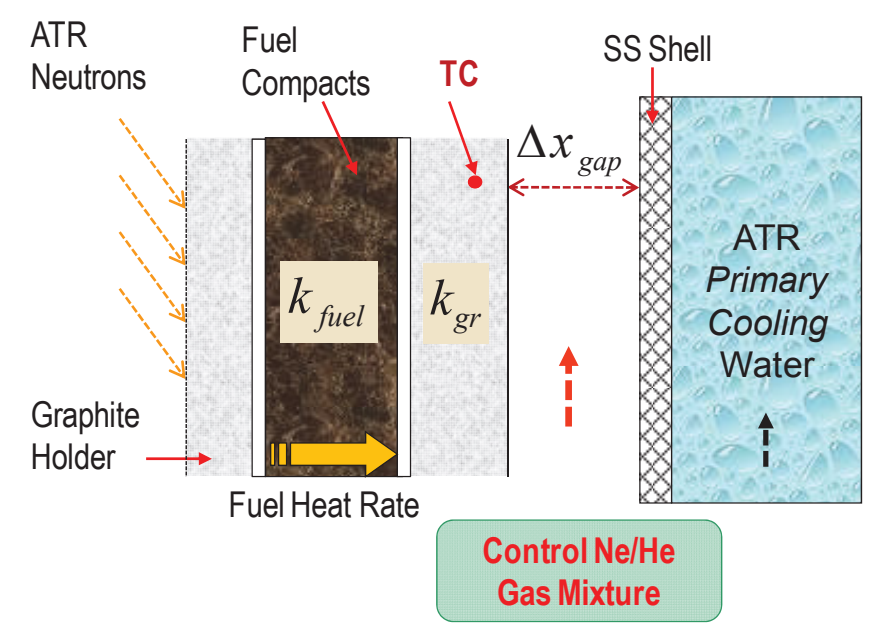

Figure S1. Physical sketch of the axial cut of an AGR-1 capsules.

Early analysis of thermocouple data indicated that they performed reliably during the beginning cycles of irradiation. Therefore, the thermal models for AGR-1 capsules were calibrated by varying the emissivities of surfaces of the graphite holder and stainless-steel retainer to best match temperatures at TC locations with actual TC measurements during this portion of the irradiation. The residuals are the differences between measured and calculated temperatures for operational TCs. Continued monitoring and analysis of residuals beyond the calibration period show a pattern around and near zero for at least one TC in every capsule, except Capsule 6. This suggests negligible model bias and therefore the uncertainty quantification performed here relies solely on the model input parameters. For Capsule 6, a bias is present but is largely compensated for by the $10 \%$ bias identified as a portion of the fuel heat rate uncertainty (Table S1).

Additionally, $\log$ of release-to-birth ratio $(\mathrm{R} / \mathrm{B})$ is a fuel temperature dependent parameter, and an analysis of the relationship between calculated fuel temperature and $\log (\mathrm{R} / \mathrm{B})$ was performed. The excellent fit in trend over the entire range of capsule thermal conditions throughout the AGR-1 irradiation provide confidence that the thermal model has appropriately included all important physical phenomena occurring in the capsule. This supports the assumption of negligible model bias and the decision to base the uncertainty analysis solely on model input parameters.

\section{S3. Input Parameter Uncertainty}

The uncertainties of the input parameters of interest in the thermal model were estimated by VHTR $\mathrm{R} \& \mathrm{D}$ program experts and are presented in Table S-1 along with the basis for the estimates.

Table S1. Uncertainties of the most significant parameters of the AGR-1 thermal model

\begin{tabular}{|c|c|c|c|}
\hline Input parameter & $\begin{array}{c}\text { Random } \\
(\%)\end{array}$ & $\begin{array}{l}\text { Bias } \\
(\%)\end{array}$ & Comments \\
\hline Control gap distance & $\begin{array}{l}3.5- \\
10.7\end{array}$ & up to 25 & $\begin{array}{l}\text { Random: } \sim 1 \text { mil fabricated tolerance. } \\
\text { Bias: time dependent because of shrinking and swelling. } \\
\text { The graphite shrinkage and swelling are proportional to the } \\
\text { reaction rate in the graphite leading to the physics-based } \\
\text { linear gas gap model. The model is justified by significant } \\
\text { correlation between R/B and fuel temperature profiles. }\end{array}$ \\
\hline Neon fraction & 3 & 0 & Random: $\sim 1 \mathrm{sccm}$ flow rate tolerance. \\
\hline Fuel heat rate & 2.5 & +10 for & Random: good fit between predicted and PIE fuel burnup \\
\hline
\end{tabular}




\begin{tabular}{|l|c|c|l|}
\hline Input parameter & $\begin{array}{c}\text { Random } \\
(\%)\end{array}$ & $\begin{array}{c}\text { Bias } \\
(\%)\end{array}$ & \multicolumn{1}{c|}{ Comments } \\
\hline & Capsule 6 & $\begin{array}{l}\text { (less than 10\%) lead to small heat uncertainty. } \\
\text { Bias: ATR axial depletion effects not accounted for in } \\
\text { structure (absorbing) above the core. These affect mostly } \\
\text { Capsule 6. }\end{array}$ \\
\hline Graphite conductivity & 15 & 0 & $\begin{array}{l}\text { Additional conductivity data for the test graphite allows a } \\
\text { lower uncertainty estimate for graphite than for fuel. }\end{array}$ \\
\hline Fuel conductivity & 20 & 0 & $\begin{array}{l}\text { Fuel conductivity values used in the model are based on } \\
\text { historic German data corrected for packing fraction and } \\
\text { matrix density. }\end{array}$ \\
\hline
\end{tabular}

\section{S4. Input Parameter Sensitivity}

The parameter sensitivity analysis of the thermal model is performed to determine the sensitivity coefficients of the most influential variables. Capsule 4 and Capsule 6 data is used for the sensitivity analysis and is assumed to be representative of the other capsules. A sensitivity coefficient describes how the model predicted temperature would be influenced by changes in an input parameter. The overall uncertainty of the model output increases as the absolute sensitivity coefficient of an input parameter increases.

The sensitivity analysis performed here went beyond the traditional local sensitivity. Using experimental design, analysis of pairwise interactions of model parameters was performed to establish sufficiency of the first-order (linear) expansion terms in the uncertainty propagation formula (Eq. S1). An interpolation scheme over the input parameter domain was then used to obtain time-dependent sensitivity over the test campaign duration. This allows computation of uncertainty for the predicted fuel temperatures and the predicted graphite temperatures at TC locations over the whole AGR-1 irradiation period.

The parameter sensitivity coefficients for AGR-1 temperature predictions as function of Effective Full Power Day (EFPD) are presented in Figure S2 and the following conclusions are drawn:

- The sensitivity coefficients of fuel fission heat rate (blue bars in Figure S2) and neon fraction (green bars in Figure S2) are highest (up to 0.7) for all predicted temperatures of interest (volume-average fuel, peak fuel, and TC) for all six capsules.

- The sensitivity coefficients of control gas gap (red bars in Figure S2) ranged from 0.2 to 0.4. These sensitivities reach their highest values for temperatures of the peripheral TCs, especially for the middle capsules (Capsules 3 and 4) which have the smallest gas gaps. The gap sensitivity is lower $(\sim 0.2)$ for fuel temperatures (volume-average and peak) and center TCs.

- The absolute values of the sensitivity coefficients of fuel compact thermal conductivity (orange bars in Figure S2) and graphite holder thermal conductivity (purple bars in Figure S2) ranged from 0.003 to 0.2 . The conductivity sensitivity coefficients are near zero for peripheral TC temperatures (i.e., the peripheral TC temperatures are insensitive to variations of fuel conductivity and graphite holder conductivity), but higher for fuel and center TC temperatures. 


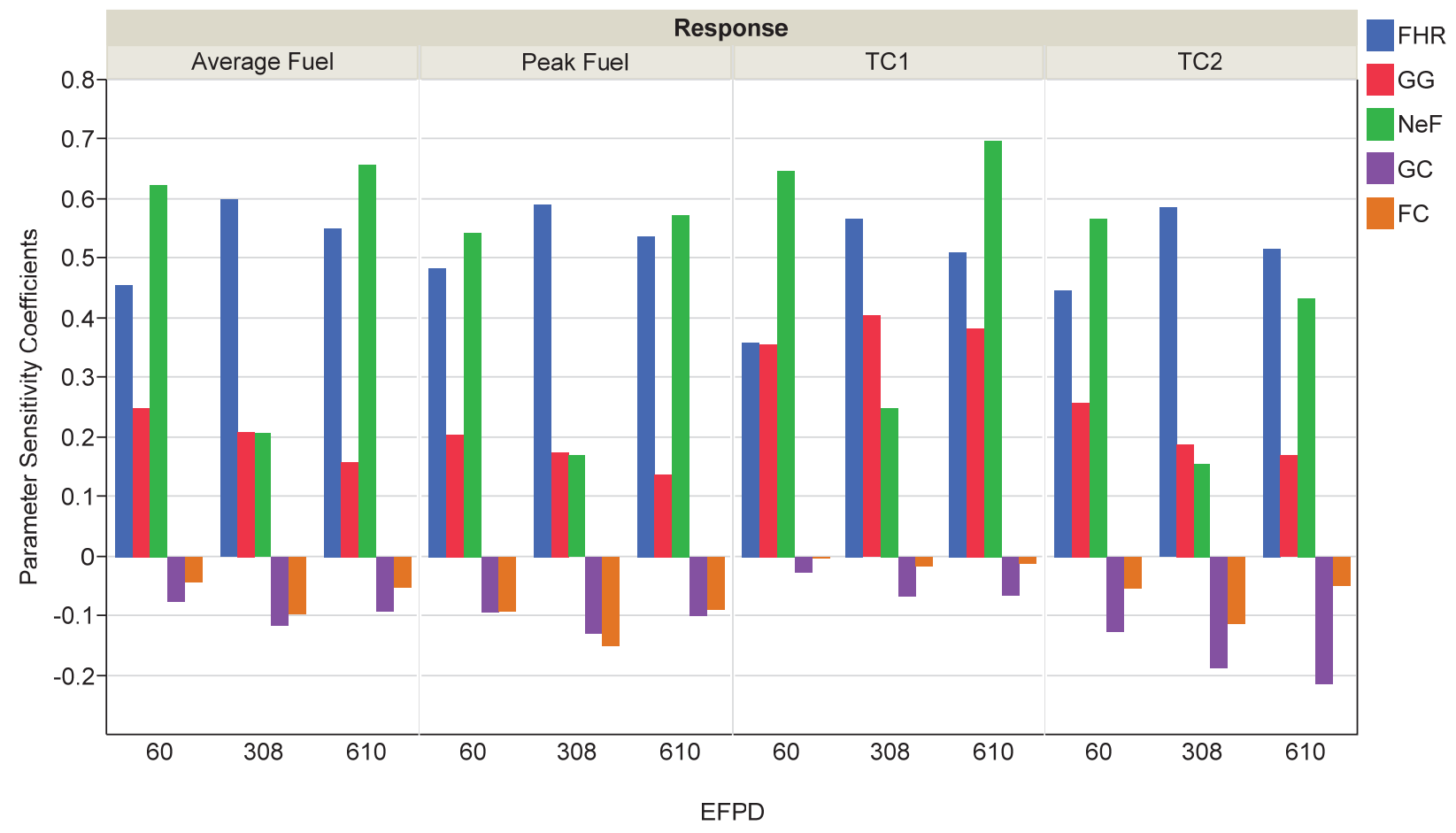

Figure S2. Parameter sensitivity coefficients for fuel and TC temperatures in Capsule 4.

\section{S5. Combining Parameter Sensitivity and Uncertainty}

The overall uncertainty of a calculated temperature in terms of variance is obtained through propagation of model parameter uncertainty as the summation of the parameter variances weighted by the squares of their sensitivity coefficients (Eq. S1). Thus, the effect of a parameter on the model prediction variation is a product of input uncertainty and the sensitivity coefficient. The most significant factors contributing to overall uncertainty of the AGR-1 temperature predictions are shown in Figure S3 for Capsule 6 and Figure S4 for Capsule 4. (The results for Capsules 1, 2, 3, and 5 are similar to the Capsule 4 results.) The following conclusions are drawn:

- For the top capsule, Capsule 6, the positive $10 \%$ bias in the fuel fission heat rate drives the overall uncertainties (as much as $90 \%$ of the overall variance, as shown by brown dots in Figure S3) of all predicted temperatures (TC, volume-average, and peak).
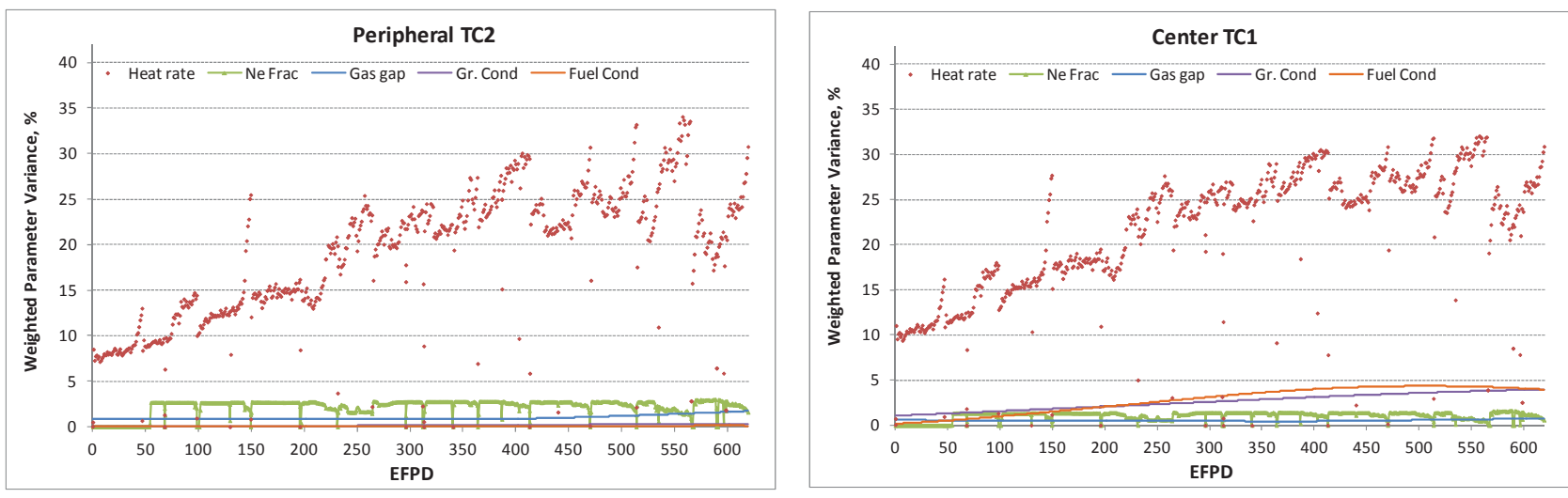

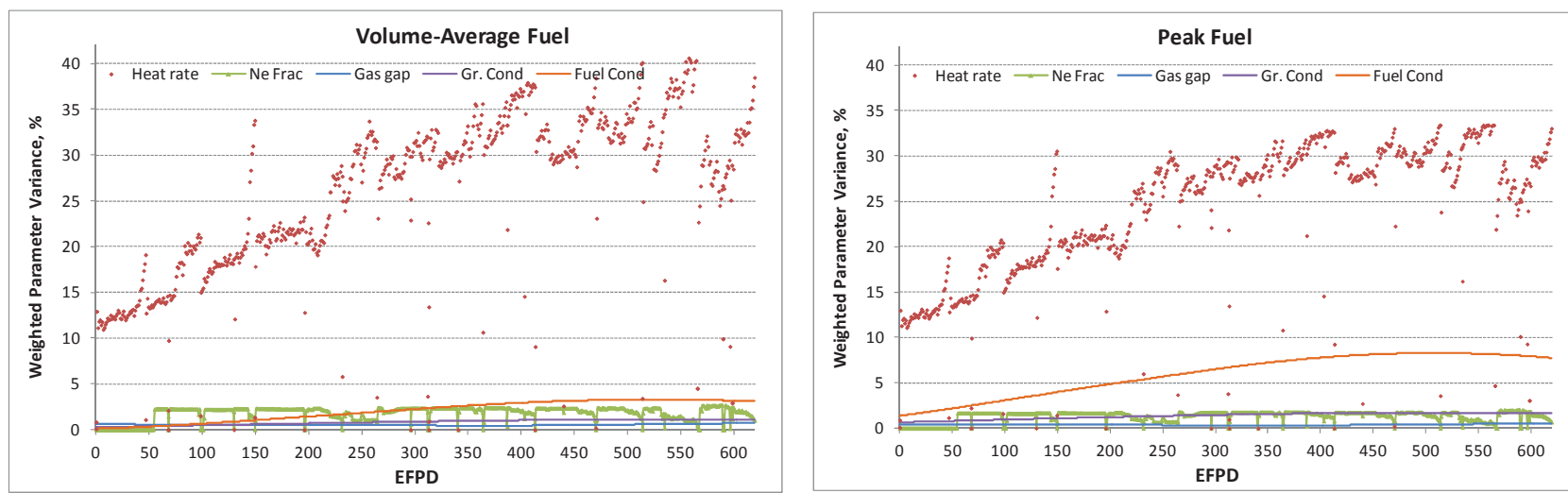

Figure S3. Daily weighted parameter variances for temperatures in the top Capsule 6.

- For the other capsules, the most influential factor depends on the temperature parameter as follows:

- For volume-average fuel temperature and peripheral TC temperature, the control gas gap is the most influential factor on overall uncertainty (as shown by the darker blues lines in the two left graphs of Figure S4), especially at the beginning (due to higher gap sensitivity) and the end (due to higher gap uncertainty).

- For center TC temperature, the graphite holder thermal conductivity, which has the second largest uncertainty of $15 \%$ and sensitivity coefficients as much as -0.2 , is the most influential factor for most of the irradiation (purple line in the top right graph of Figure S4).

- For peak fuel temperature, the most influential factor is the fuel compact thermal conductivity (light blue line on the bottom right graph), which has the largest uncertainty (20\%) and a large sensitivity coefficient (up to -0.15). This is followed by the gas gap (darker blue line) and then the graphite holder thermal conductivity (purple line).
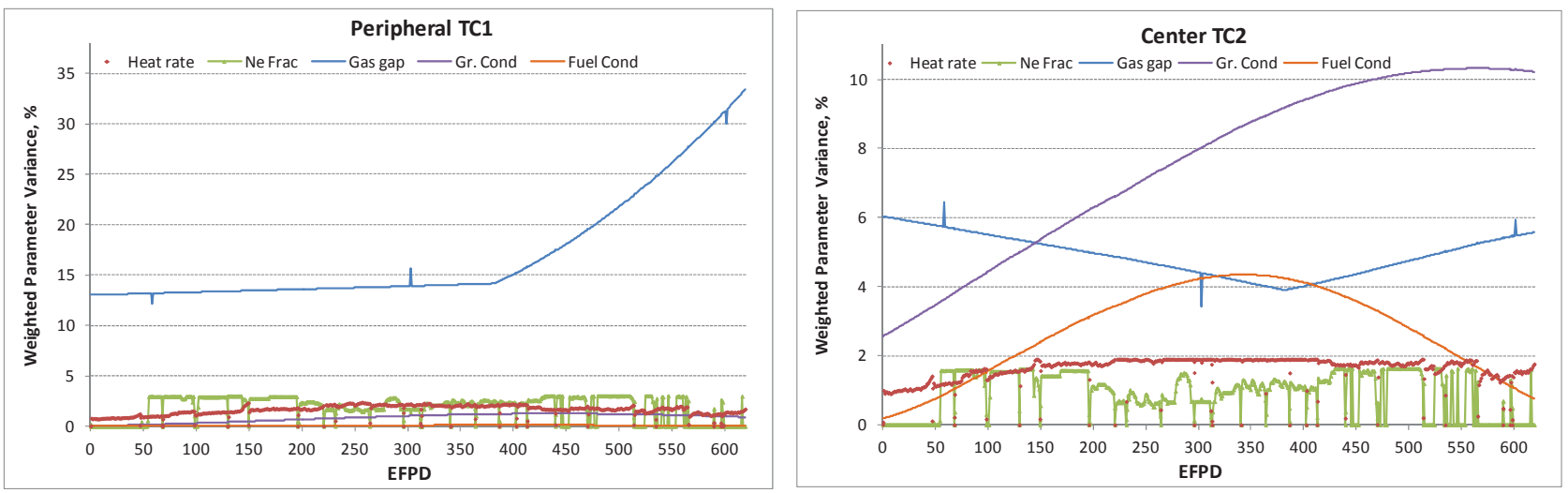

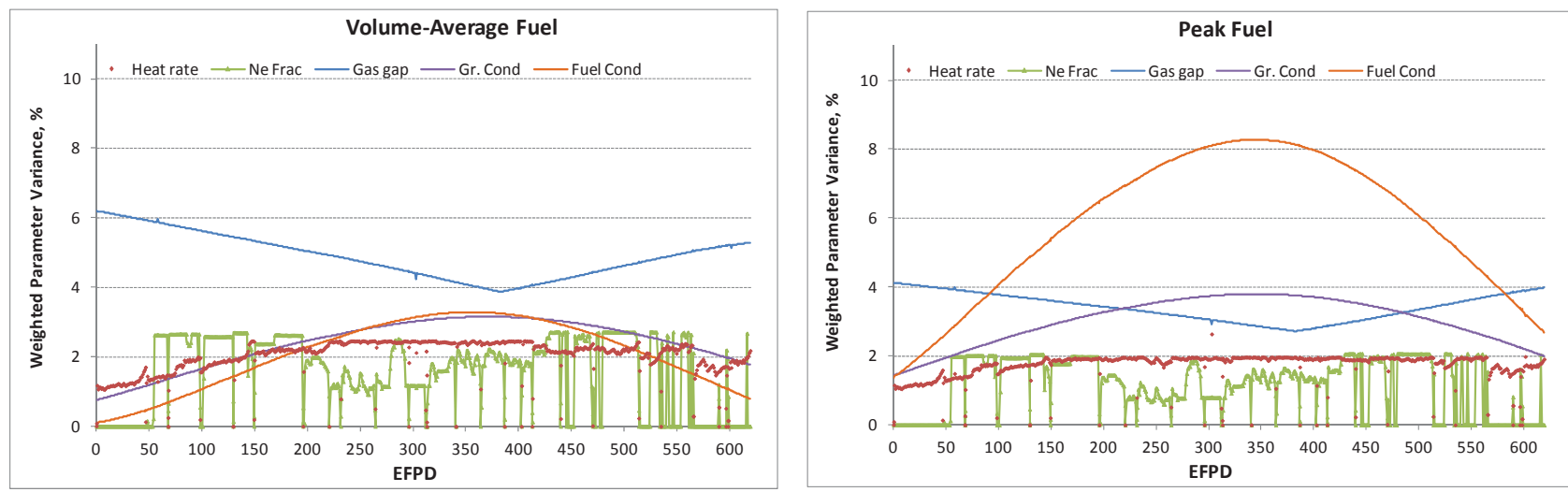

Figure S4. Daily weighted parameter variances for temperatures in the middle Capsule 4.

\section{S6. Overall Uncertainty}

The overall uncertainty in the calculated temperatures for AGR-1 ranged from $2.0 \%$ to $6.5 \%$, depending on irradiation time (thermal conditions), capsule, and the temperature parameter being predicted (peak, volume-average, or TC). Table S2 presents temperatures and their relative and absolute standard deviations for time-average volume-average and time-average peak fuel temperatures at the end of irradiation for all six capsules. The overall uncertainties for instantaneous volume-average and instantaneous peak fuel temperatures in Capsule 4, as a function of EFPD, are presented in Figure S5. The overall uncertainties for time-average volume-average and time-average peak fuel temperatures in Capsule 4 are presented in Figure S5. Result highlights are:

- For temperatures at TCs, the overall uncertainty ranged from $2 \%$ to $6.5 \%$. The highest relative uncertainty at the end of AGR-1 is $6.5 \%\left(\sim 48{ }^{\circ} \mathrm{C}\right)$ for the peripheral TC in Capsule 3, and $6 \%$ $\left(\sim 45^{\circ} \mathrm{C}\right)$ for the peripheral TCs in Capsules 4 and 6 . This uncertainty of predicted temperatures at the peripheral TCs is caused mainly by the increasing uncertainty of the control gas gap distance, especially for the middle capsules at the end of irradiation. The increase of gap uncertainty has more effect on the temperature uncertainty of peripheral TCs than on the uncertainty of the center TC. The adequacy of basing the uncertainty analysis on model input parameters is confirmed because the overall model uncertainty of TC temperature prediction is consistent with TC residual variation that ranged from $19{ }^{\circ} \mathrm{C}$ to $54^{\circ} \mathrm{C}$ for one TC in each capsule.

- For fuel temperatures, the volume-average temperature uncertainties are slightly lower than peak temperature uncertainties such that: (i) the relative uncertainty ranged from $3 \%$ to $4 \%$ for volumeaverage temperatures; and (ii) ranged from $3 \%$ to $5 \%$ for peak temperatures (up to $\sim 65{ }^{\circ} \mathrm{C}$ ). The fuel temperature uncertainty reaches its highest value at the time when the sensitivity coefficients of fuel and graphite thermal conductivity are highest leading to large variation of the calculated fuel temperatures.

- The time-average volume-average fuel temperature uncertainty reaches $3.7 \%\left(\sim 40{ }^{\circ} \mathrm{C}\right)$ and the time-average peak fuel temperature uncertainty reaches $4.4 \%\left(\sim 52^{\circ} \mathrm{C}\right)$ after two thirds of the irradiation. 
Table S2. Temperatures and uncertainty for time-average fuel temperatures at the end of AGR-1.

\begin{tabular}{|l|c|c|c|c|c|c|}
\hline \multirow{2}{*}{ Capsule } & \multicolumn{2}{|c|}{ time-average volume-average fuel } & \multicolumn{3}{|c|}{ time-average peak fuel } \\
\cline { 2 - 7 } & $\mathrm{T},{ }^{\circ} \mathrm{C}$ & $\sigma_{\mathrm{T}}, \%$ & $\sigma_{\mathrm{T}},{ }^{\circ} \mathrm{C}$ & $\mathrm{T},{ }^{\circ} \mathrm{C}$ & $\sigma_{\mathrm{T}}, \%$ & $\sigma_{\mathrm{T}},{ }^{\circ} \mathrm{C}$ \\
\hline Capsule 6 & 1088 & 5.014 & 55 & 1204 & 5.012 & 60 \\
Capsule 5 & 1023 & 3.700 & 38 & 1157 & 4.301 & 50 \\
Capsule 4 & 1070 & 3.743 & 40 & 1202 & 4.327 & 52 \\
Capsule 3 & 1029 & 3.777 & 39 & 1162 & 4.330 & 50 \\
Capsule 2 & 1003 & 3.830 & 38 & 1141 & 4.379 & 50 \\
Capsule 1 & 1055 & 3.165 & 33 & 1178 & 3.776 & 45 \\
\hline
\end{tabular}

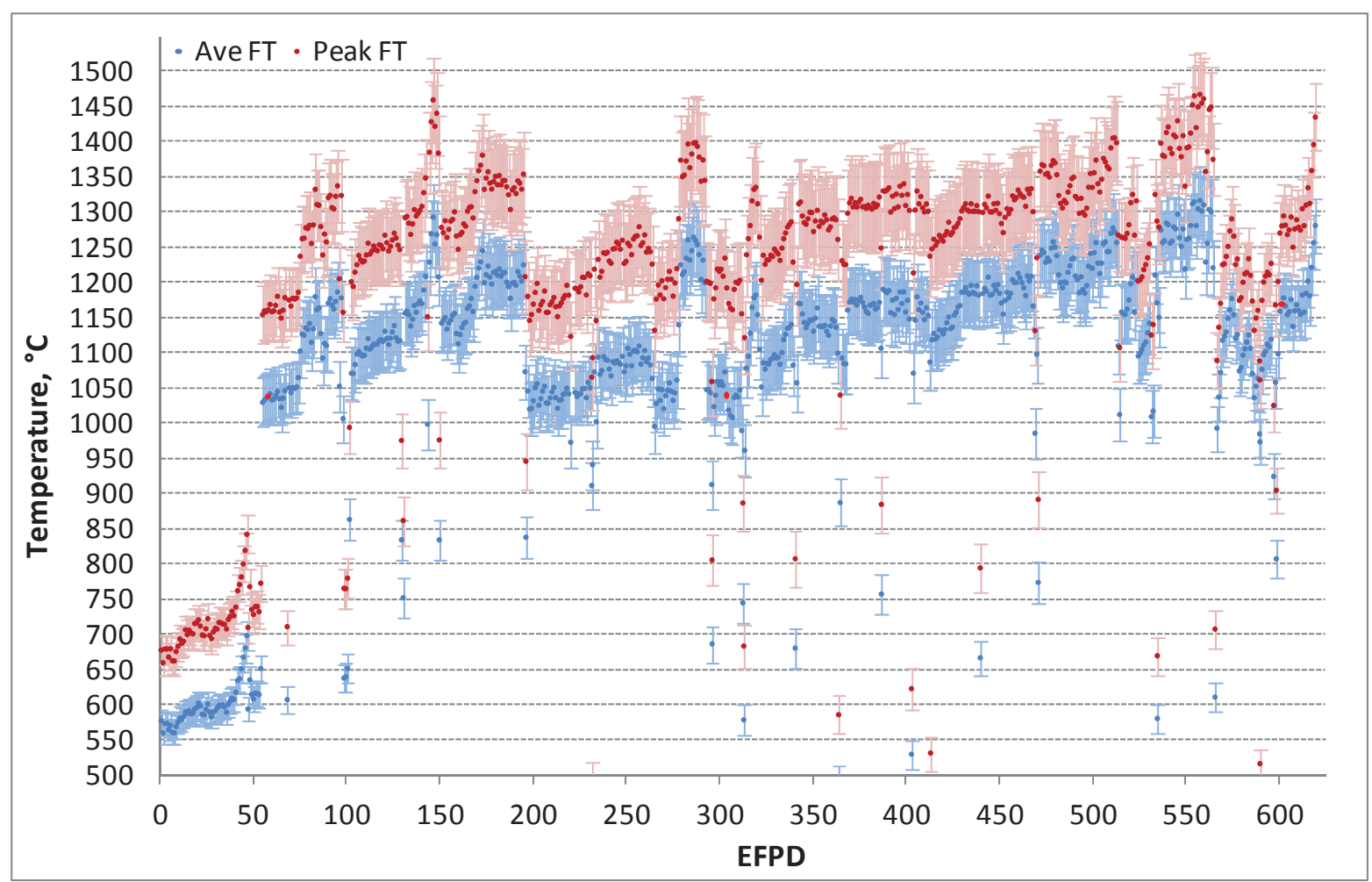

Figure S5. Instantaneous volume-average and peak fuel temperature uncertainties in Capsule 4. 


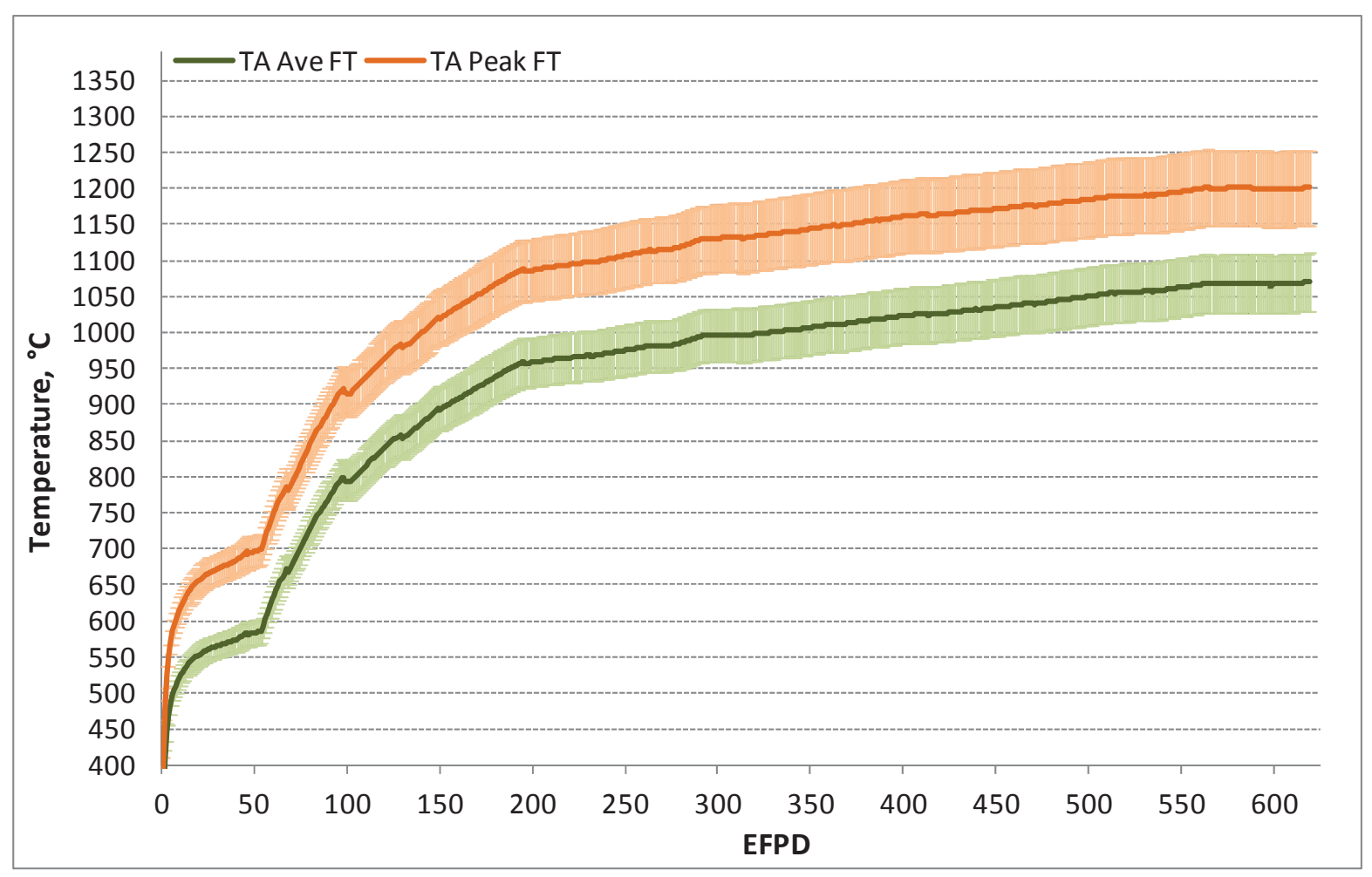

Figure S6. Time-average volume-average and time-average peak fuel temperature uncertainties in Capsule 4.

\section{S7. Report Structure}

The report is organized into four sections and a conclusion:

- Section 1 introduces the AGR Fuel Development and Qualification program and overviews AGR-1 measured data, AGR-1 test configuration and test procedure, and thermal simulation.

- Section 2 describes the estimation of uncertainties and sensitivities for the thermal model input parameters. This includes parameter uncertainties based on expert judgment, sensitivity analysis of input parameters, and the estimation of correlation coefficients for pairs of input parameters.

- Section 3 describes the propagation of uncertainties and sensitivities for estimation of the overall uncertainty for the daily volume-average and peak fuel temperatures, daily average temperatures at TC locations, and time-average volume-average and time-average peak fuel temperatures.

- Section 4 presents the results of the uncertainty analysis for each of the six AGR-1 capsules and discusses the parameters driving the uncertainty.

- The conclusion summarizes important findings of the uncertainty quantification and identifies possible areas of improvement. 


\section{CONTENTS}

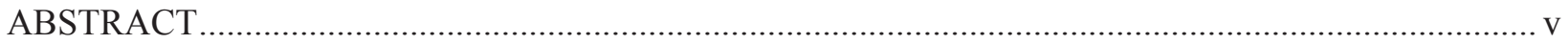

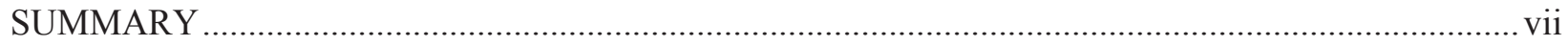

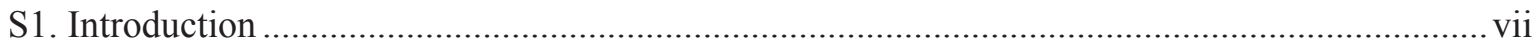

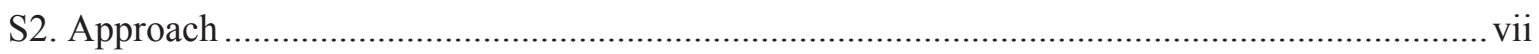

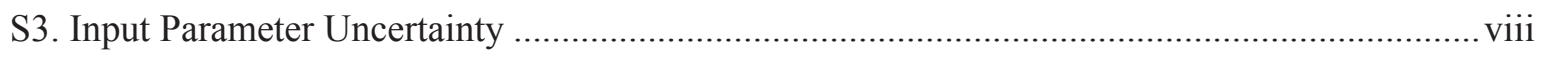

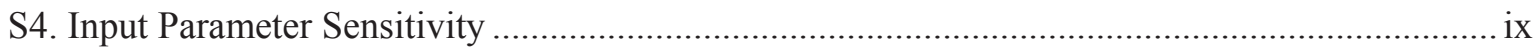

S5. Combining Parameter Sensitivity and Uncertainty …........................................................

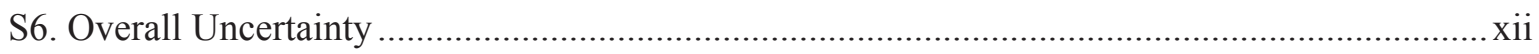

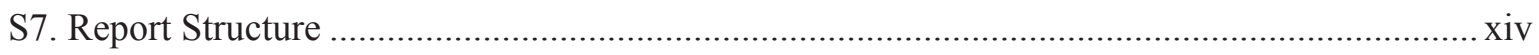

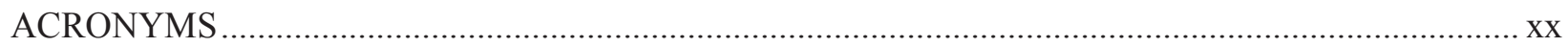

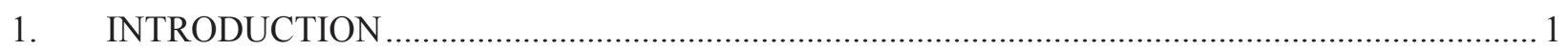

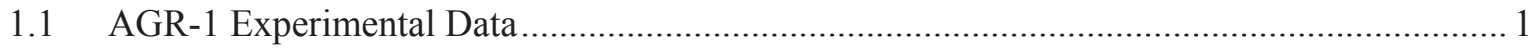

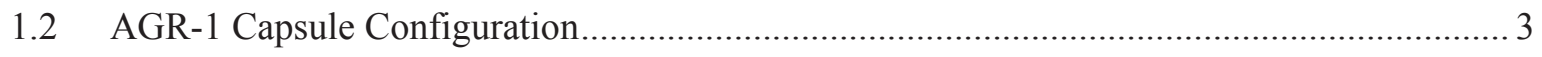

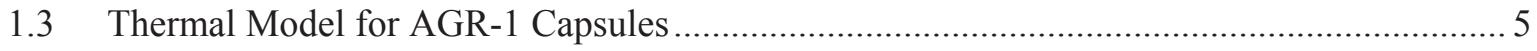

2. ESTIMATION OF INPUT PARAMETER UNCERTAINTIES AND SENSITIVIES …................. 7

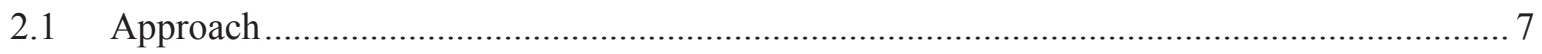

2.2 Rationale for Basis of Uncertainty Analysis ............................................................ 7

2.2.1 Improved Agreement between Calculated and Measured TC Readings..................... 7

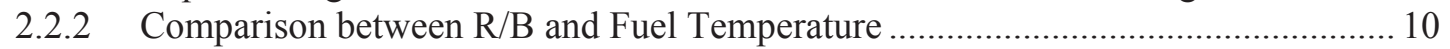

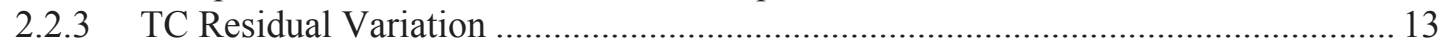

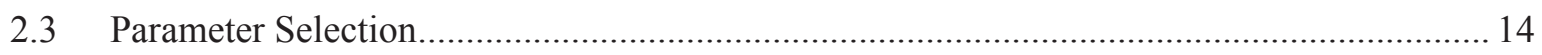

2.4 Input Parameter Uncertainties Based on Expert Judgment............................................... 15

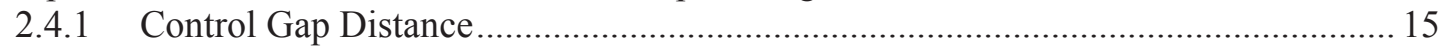

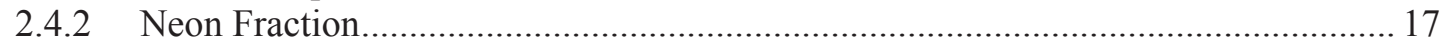

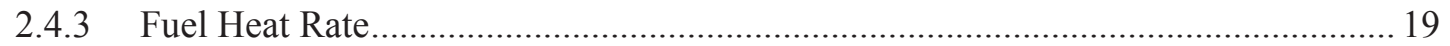

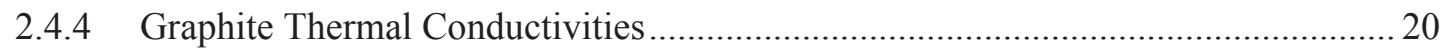

2.4.5 Fuel Compact Thermal Conductivities .................................................................. 21

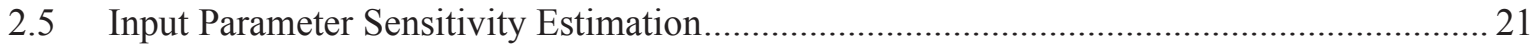

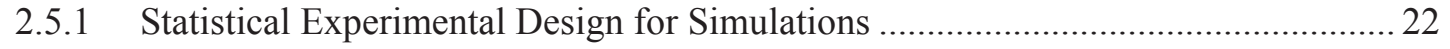

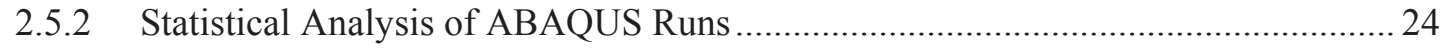

2.5.3 Interpolation of Sensitivity Coefficients for Entire AGR-1 Irradiation ..................... 28

2.6 Correlation Coefficients of Thermal Model Input Parameters............................................. 32

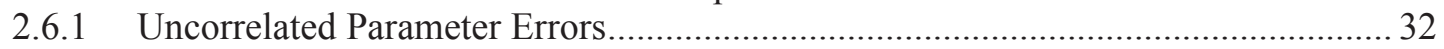

2.6.2 Correlated Parameter Errors............................................................................... 32

3. PROPAGATION OF PARAMETER UNCERTAINTIES AND SENSITIVITIES ........................ 35

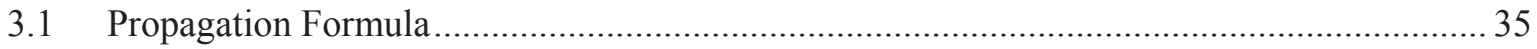

3.2 Uncertainty Propagation for Daily Average Temperatures................................................ 35

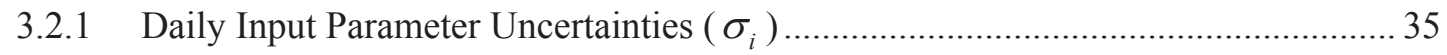




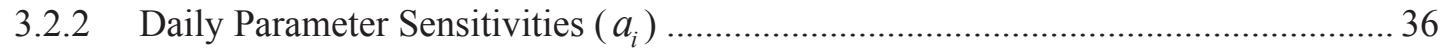

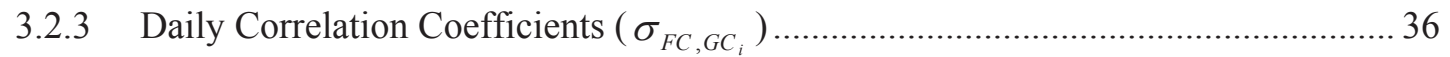

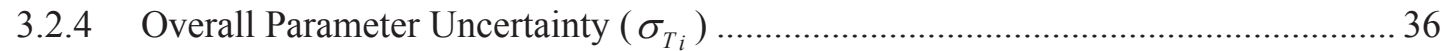

3.3 Uncertainty Quantification for Time Average (TA) Temperatures .................................... 37

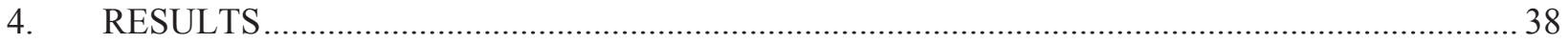

4.1 Temperature Uncertainty Results for Capsule 6 ............................................................. 38

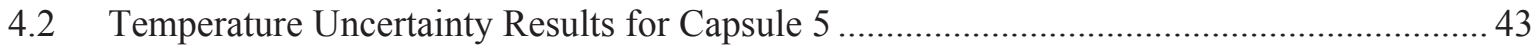

4.3 Temperature Uncertainty Results for Capsule 4 ............................................................... 48

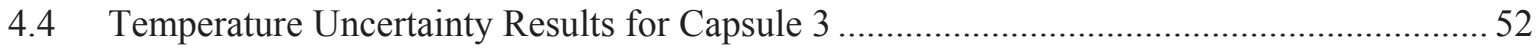

4.5 Temperature Uncertainty Results for Capsule 2 ................................................................ 57

4.6 Temperature Uncertainty Results for Capsule 1 ............................................................. 61

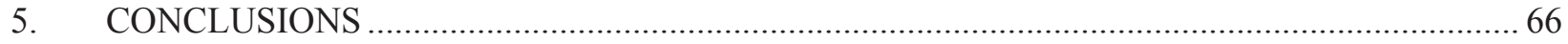

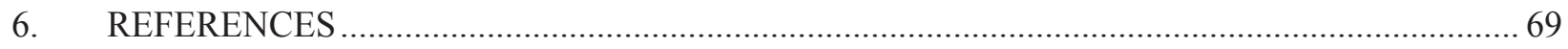

\section{FIGURES}

Figure S2. Parameter sensitivity coefficients for fuel and TC temperatures in Capsule 4........................

Figure S5. Instantaneous volume-average and peak fuel temperature uncertainties in Capsule 4.............xiii

Figure S6. Time-average volume-average and time-average peak fuel temperature uncertainties in

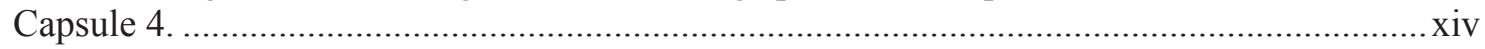

Figure 1. Graphical summary of Capsule 4 data during AGR-1 test. ...................................................... 3

Figure 2. Schematic of a radial cut of an AGR-1 capsule ................................................................... 4

Figure 3. Physical sketch of the axial cut of an AGR-1 capsules. ............................................................ 4

Figure 4. Temperature distribution in cutaway view of three fuel stacks. ............................................... 6

Figure 5. The TC residuals of the original (run 1) and updated (run 2) thermal models for

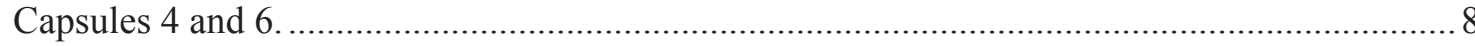

Figure 6. Difference between measured and predicted thermocouple temperatures. ................................9

Figure 7. Capsule 4 normalized volume average fuel temperatures, normalized $\log (R / B)$ and their

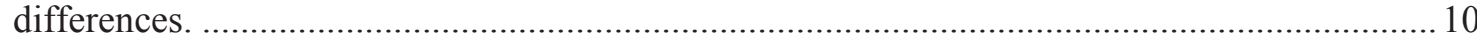

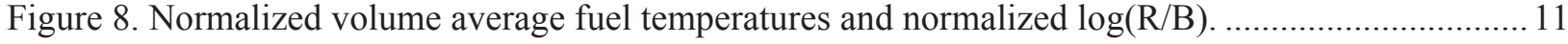

Figure 9. Differences between normalized volume average fuel temperatures and normalized

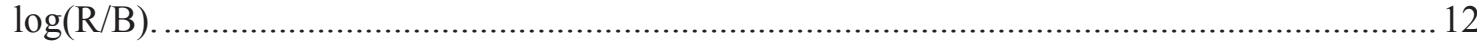

Figure 10. Histograms of temperature residuals of a peripheral TC of six capsules. ............................. 13

Figure 11. Tornado plot of peak fuel temperature sensitivity.............................................................. 14

Figure 12. The initial and end gap distance with an error bar of 1 mil for six AGR-1 capsules. .............. 16

Figure 13. Relative change in outer diameter of graphite holders. ......................................................... 17 
Figure 14. Neon fraction uncertainty and the trend line.

Figure 15. Smooth plots of daily capsule average volumetric heat rates in compacts versus EFPD.

Figure 16. Ratio of irradiated over unirradiated graphite thermal conductivity $\left(\mathrm{k}_{\mathrm{irr}} / \mathrm{k}_{\mathrm{o}}\right)$ varying with temperature and dpa.. 20

Figure 17. Fuel compact thermal conductivity varying with fluence and temperature.............................2

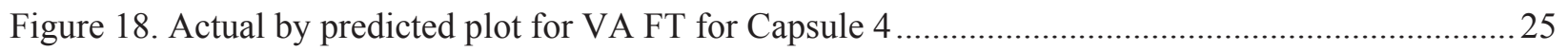

Figure 19. Leverage plots for main effects and the square term of Ne fraction for VA FT.......................26

Figure 20. Prediction profiles of functions for fuel and TC temperatures. ..............................................26

Figure 21. Parameter estimate plots for fuel and TC temperatures in Capsule 4....................................28

Figure 22. Heat rate sensitivity for TCs and fuel temperatures as function of heat rate..........................29

Figure 23. Neon fraction sensitivity for TCs and fuel temperatures as function of neon fraction...............30

Figure 24. Gas gap sensitivity for TCs and fuel temperatures as function of gap distance. ...................... 30

Figure 25. Graphite conductivity sensitivity for TCs and fuel temperatures as function of fluence. ..........31

Figure 26. Fuel conductivity sensitivity for TCs and fuel temperatures as function of fluence.................31

Figure 27. Scatter plot matrix of fuel temperature, $d p a$, fuel and graphite thermal conductivities. ............33

Figure 28. Correlation coefficients between graphite and fuel thermal conductivities varying with

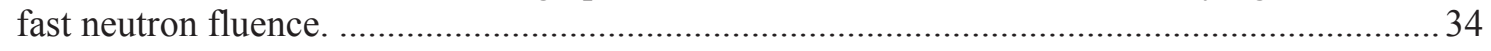

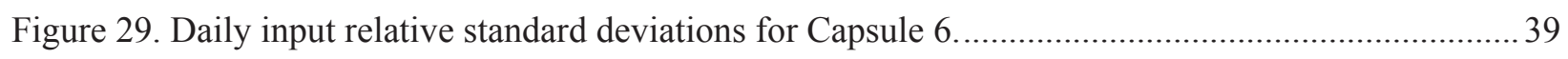

Figure 30. Daily input parameter sensitivities for temperatures in Capsule 6......................................... 40

Figure 31. Model sensitivities of graphite conductivity: Capsule 6 actual data (empty symbols) versus predicted (cross symbol) based on Capsule 4 actual data (solid symbols)..................... 40

Figure 32. Daily relative standard deviations of predicted TC and fuel temperatures in Capsule 6..........41

Figure 33. Daily standard deviations of predicted TC and fuel temperatures in Capsule 6......................41

Figure 34. Model temperature and standard deviation of TCs in Capsule 6........................................... 42

Figure 35. Model temperature and standard deviation of daily averaged fuel temperatures in Capsule 6. 42

Figure 36. Model temperature and standard deviation of time-average fuel temperatures in Capsule 6. 43

Figure 37. Daily input relative standard deviations for Capsule 5 ..................................................... 44

Figure 38. Daily input parameter sensitivities for temperatures in Capsule 5 ........................................ 45

Figure 39. Daily relative standard deviations of predicted TC and fuel temperatures in Capsule 5..........45

Figure 40. Daily standard deviations of predicted TC and fuel temperatures in Capsule 5......................46

Figure 41. Model temperature and standard deviation of TCs in Capsule 5...........................................46

Figure 42. Model temperature and standard deviation of daily fuel temperatures in Capsule 5................47

Figure 43. Model temperature and standard deviation of time-average fuel temperatures in Capsule 5. 47 
Figure 44. Daily input relative standard deviations for Capsule 4 ......................................................... 49

Figure 45. Daily input parameter sensitivities for temperatures in Capsule 4. ......................................... 49

Figure 46. Capsule 4 calculated TC and fuel temperature (FT) relative standard deviations....................50

Figure 47. Capsule 4 calculated TC and fuel temperature (FT) standard deviations...............................50

Figure 48. Model temperature and standard deviation of TCs in Capsule 4 ...........................................51

Figure 49. Model temperature and standard deviation of fuel compacts in Capsule 4............................51

Figure 50. Model temperature and standard deviation of time-average fuel temperatures in

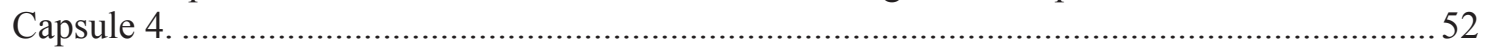

Figure 51. Daily input relative standard deviations for Capsule 3 ..................................................... 53

Figure 52. Daily input parameter sensitivities for temperatures in Capsule 3......................................54

Figure 53. Daily relative standard deviations of predicted TC and fuel temperatures in Capsule 3..........54

Figure 54. Daily standard deviations of predicted TC and fuel temperatures in Capsule 3......................55

Figure 55. Model temperature and standard deviation of TCs in Capsule 3 ...........................................55

Figure 56. Model temperature and standard deviation of daily fuel temperatures in Capsule 3................56

Figure 57. Model temperature and standard deviation of time-average fuel temperatures in

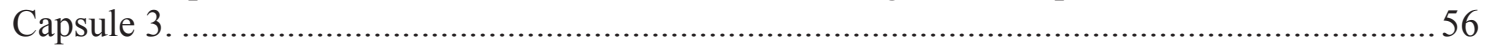

Figure 58. Daily input relative standard deviations for Capsule 2 ....................................................5

Figure 59. Daily input parameter sensitivities for temperatures in Capsule 2. ......................................58

Figure 60. Daily relative standard deviations of predicted TC and fuel temperatures in Capsule 2...........59

Figure 61. Daily standard deviations of predicted TC and fuel temperatures in Capsule 2......................59

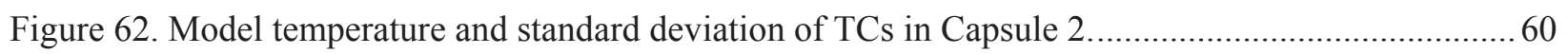

Figure 63. Model temperature and standard deviation of daily fuel temperatures in Capsule 2................60

Figure 64. Model temperature and standard deviation of time-average fuel temperatures in

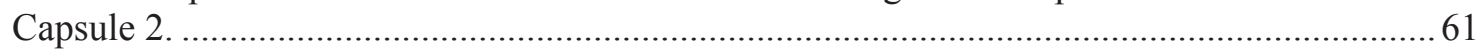

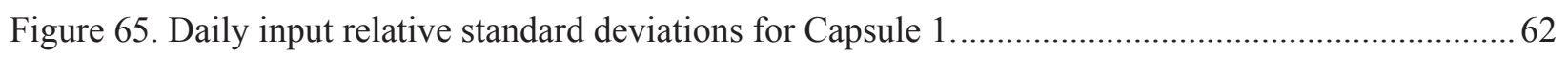

Figure 66. Daily input parameter sensitivities for temperatures in Capsule 1.........................................63

Figure 67. Daily relative standard deviations of predicted TC and fuel temperatures in Capsule 1..........63

Figure 68. Daily standard deviations of predicted TC and fuel temperatures in Capsule 1......................64

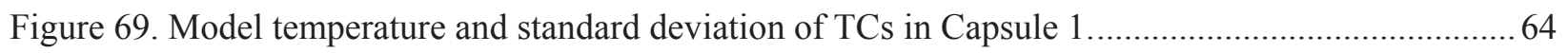

Figure 70. Model temperature and standard deviation of daily fuel temperatures in Capsule 1................65

Figure 71. Model temperature and standard deviation of time-average fuel temperatures in Capsule 1. 


\section{TABLES}

Table 1. Uncertainties of the most significant parameters of the AGR-1 thermal model......................... 15

Table 2. Control gap distance and fabrication uncertainty for AGR-1 capsules...................................... 16

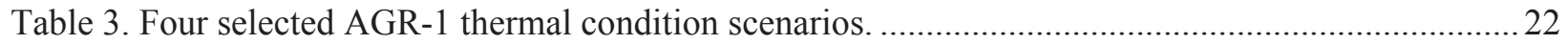

Table 4. Experimental design matrix for AGR-1 thermal model sensitivity analysis. ${ }^{\mathrm{a}}$.............................23

Table 5. Parameter estimates for VA FT, peak FT and TC temperatures................................................27

Table 6. Sensitivity coefficients of fuel heat rate for TCs, VA FT and peak FT....................................28

Table 7. Correlation coefficients between graphite and fuel thermal conductivities................................ 33

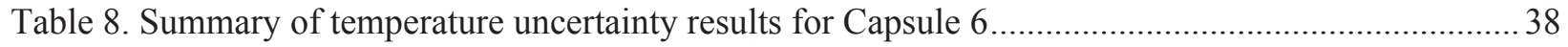

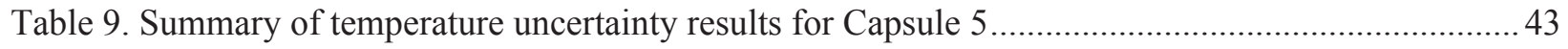

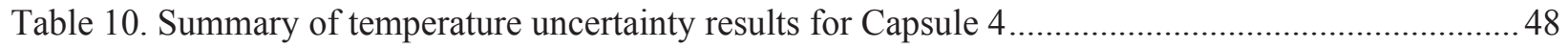

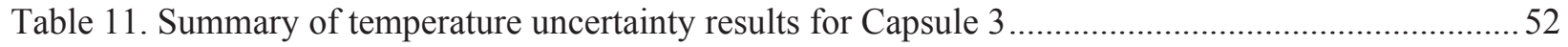

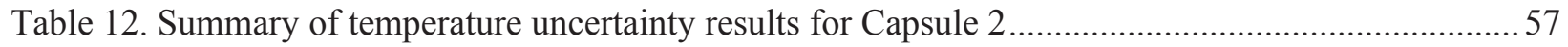

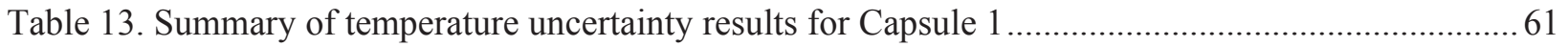




\section{ACRONYMS}

3-D three-dimensional

AGR Advanced Gas Reactor

ATR Advanced Test Reactor

dpa displacements per atom

EFPD effective full-power day

FC fuel compact

FT fuel temperature

GC graphite conductivity

GG gas gap

HR heart rate

INL Idaho National Laboratory

MFC mass flow controller

NDMAS NGNP Data Management and Analysis System

$\mathrm{NeF} \quad$ neon fraction

NGNP Next Generation Nuclear Plant

pdf probability density function

PIE post-irradiation examination

R\&D Research and Development

TA time average

TC thermocouple

TRISO tristructural-isotropic

VA volume average

VHTR Very High Temperature Reactor 


\section{Uncertainty Quantification of Calculated Temperatures for the AGR-1 Experiment}

\section{INTRODUCTION}

A series of Advanced Gas Reactor (AGR) irradiation experiments are being conducted within the VHTR R\&D Program for Next Generation Nuclear Plant (NGNP) fuel performance. The main objectives of the fuel experimental campaign are to provide the necessary data on fuel performance to support fuel process development, qualify a fuel design and fabrication process for normal operation and accident conditions, and support development and validation of fuel performance and fission product transport models and codes (Petti, 2010). The AGR test trains are multi-capsule and instrumented lead experiments. Each capsule employs fuel compacts placed in a graphite cylinder shrouded by a hafnium and stainless steel layer and capsule shell. The tests are instrumented with thermocouples (TCs) embedded in graphite blocks, and the target quantity (fuel/graphite temperature) is regulated by the helium-neon gas mixture that fills the gap volume (Maki, 2009). The AGR-1 test was inserted in the B-10 position in the Advanced Test Reactor (ATR) core at Idaho National Laboratory (INL) in December 2006 and successfully completed irradiation in November 2009, resulting in fuel irradiation for a period of nearly 3 years. Six capsules were used in the AGR-1 test. The AGR-1 data, including the experimental data collected over the course of testing (2007-2009), as well as simulation results, were qualified and stored in the NGNP Data Management and Analysis System (NDMAS) (Abbott, Hull, Pham, \& Plummer, 2010).

While no direct measurements of fuel temperature are available, the physics-based simulation codes were created to calculate ATR neutronics (and subsequently, AGR power) (Sterbentz, 2011b) and thermal conditions of the AGR experiments (Hawkes G. , 2012). The graphite temperature from TCs in AGR tests was used to calibrate and validate computer codes. The AGR simulation results collected in the NDMAS are very useful in aiding experimental data qualification, especially increasing the confidence in delineating failures of the measuring instruments (TCs) from physical mechanisms that may have shifted the system thermal response (Pham \& Einerson, 2011). Given a high rate of TC failure under harsh irradiation and thermal conditions in AGR, it is beneficial to utilize the temperature prediction results from the daily AGR power/thermal simulations to provide early detection of changes (drifting) or failure of TCs and support a more effective test control procedure. Therefore, the NDMAS organizes (links data to appropriate components such as fuel compacts, capsules, etc.) and stores output from physics-based simulation codes. These simulated data are qualified by the modelers through their Engineering Calculations and Analysis Reports.

This report focuses on the uncertainty quantification of the ABAQUS-based thermal model temperature predictions of AGR-1 capsules. The process quantifies the effect that uncertain input variables and code models have on the uncertainty distribution of code outputs. It is noted that the NGNP nuclear fuel performance involves complex physical mechanisms (e.g., fissile depletion, graphite, and fuel shrinkage) and properties (e.g., conductivity and density). Thus, the combined use of test data and simulation results requires a systematic approach to uncertainty quantification of both experimental measurements and model and numerical solutions.

\subsection{AGR-1 Experimental Data}

The AGR test trains employ fuel compacts placed in a graphite cylinder shrouded by a steel capsule. Six capsules are used in the AGR-1 test, numbered consecutively from Capsule 6 at the top to Capsule 1 at the bottom. The AGR-1 test was inserted in the B10 position located on the east side of the ATR core in December 2006 and successfully completed in November 2009, resulting in fuel irradiation for a period of nearly 3 years or 620 effective full power days (EFPD). The AGR-1 data, consisting of irradiation, 
fission product monitoring system, and post-irradiation examination (PIE) data collected over the course of irradiation (2007-2009) and PIE, were scrutinized and stored in the NDMAS. For example, the graphical plots in Figure 1 summarizing important data streams for Capsule 4 are displayed in the external website for test monitoring by NGNP program members from various organizations (INL, Oak Ridge National Laboratory, Department of Energy, etc.). The primary objective of the AGR-1 test is the shakedown of the test train (Maki, 2009). Lessons learned from thorough analysis of extensive data from this test will be important in the modification of subsequent test trains.

The tests are instrumented with TCs embedded in graphite sample holders of each capsule. These measurements will support thermal analyses of the test train, which ultimately determine fuel temperatures and support temperature control of the experiment. The target fuel compact temperature is independently regulated by the helium-neon gas mixture that fills the gap volume for each capsule. Measurements from a designated control TC provide feedback to the automated sweep gas mass flow controller (MFC) system for each capsule, which then adjusts gas blend to maintain reference temperature. The TCs have an installed accuracy of $\pm 2 \%$ of readings as required by the test specification (Maki, 2009). Qualification of TC and gas flow measurement data (Abbott, Hull, Pham, \& Plummer, 2010) is not based on specifications but rather on answers to the questions: are the data reflecting what is expected and a anomalies or trends present that require investigation

There are 17 TCs in six capsules where temperatures in the graphite holder are recorded every 10 minutes for an initial phase of the experiment, then every 5 minutes for the remaining test time. The number of TCs in capsules and the diameter of their wires are limited by space in the test train through tubes. These particular TCs were selected based on the greatest survival probability and least amount of drift in accelerated furnace tests; however, TC failures are still expected because of high temperature and high neutron fluence exposure during extended irradiation far beyond vendor specification.

Unfortunately, there is no calibration for TCs after insertion in the AGR test train, even during PIE examination. Therefore, their reading accuracy could be assessed only by comparison with the readings from other TCs in similar positions or with calculated values. The TC data are rigorously analyzed and qualified in NDMAS (Pham \& Einerson, 2011).

The fission product releases of many radionuclides, which are important criteria for test fuel performance assessment, are separately monitored for each capsule. The fission product R/B for several isotopes (e.g., $\mathrm{Kr}-85 \mathrm{M}, \mathrm{Kr}-88$, and $\mathrm{Xe}-135$ in Panel 6 of Figure 1) are also important in capsule thermal analysis because of their close relationship with temperature. This fission product $\mathrm{R} / \mathrm{B}$ is the ratio between measured radionuclide release rates (in atoms $/ \mathrm{sec}$ ) and calculated radionuclide birthrates. These data are captured every 8 hours and can be used to confirm changes in temperature. Gross radiation monitors continuously measure the sweep gas from each capsule to indicate fuel particle failures. 


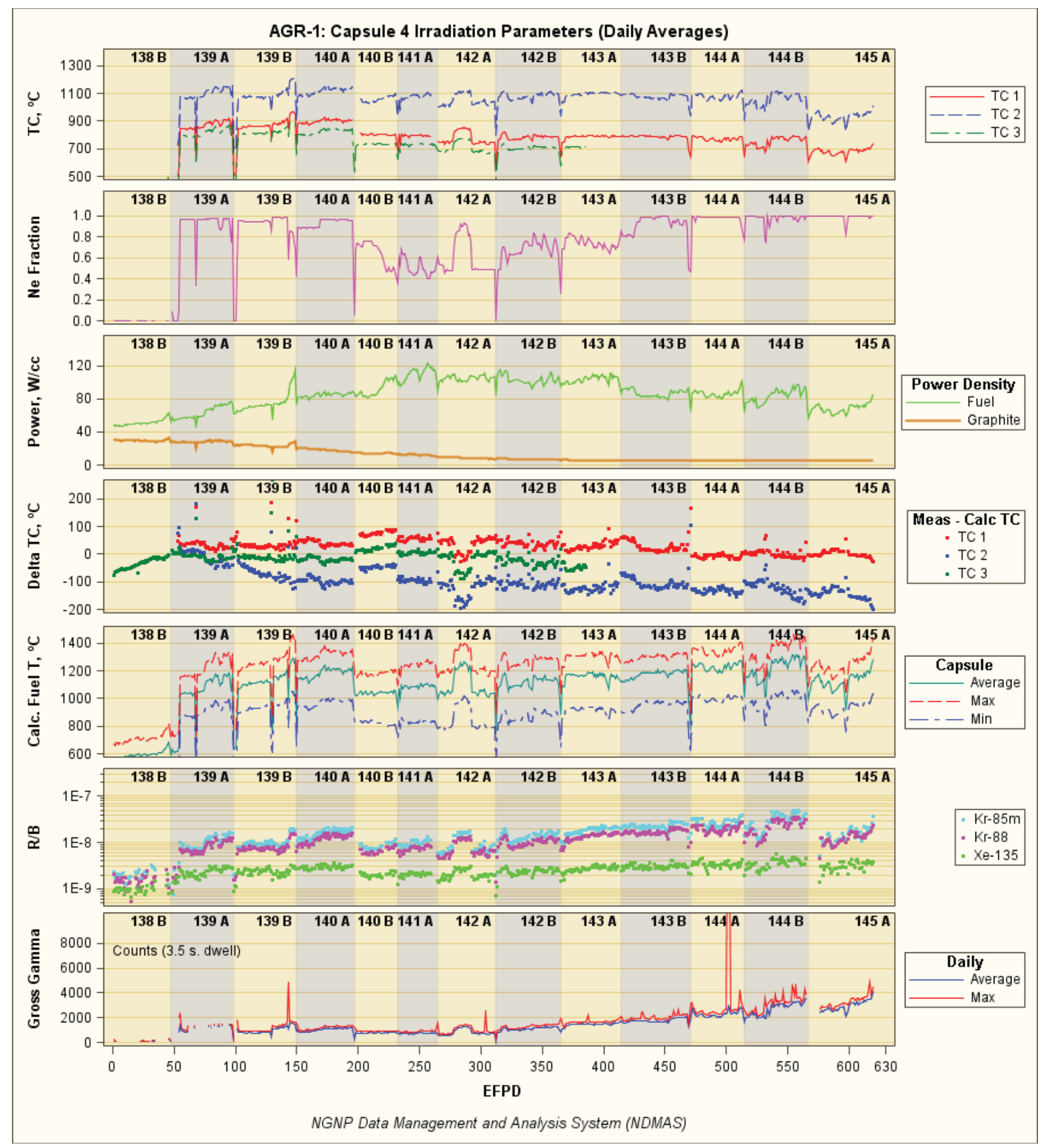

Figure 1. Graphical summary of Capsule 4 data during AGR-1 test.

\subsection{AGR-1 Capsule Configuration}

The six capsules used in the AGR-1 test train are numbered consecutively from Capsule 6 at the top to Capsule 1 at the bottom. Each AGR capsule contains twelve fuel compacts stacked in three vertical columns of four compacts per stack, which are placed in a graphite cylinder shrouded by a hafnium and stainless steel layer and housed in a capsule body (Figure 2). The TC and gas-line locations are also shown in Figure 2. 
Figure 3 is a physical sketch representing the thermal model and parameters of each capsule in AGR1 test train. The ATR primary cooling water is the ultimate heat sink for each capsule. The fission power generated in the fuel compact and graphite sample holder is mainly conducted out to the ATR primary cooling water through the two gas gaps: one between the fuel compact and the graphite holder, and one between the graphite holder and the stainless steel shell. The tests are instrumented with TCs embedded in graphite blocks to measure the lower temperature in the graphite. The independently controlled heliumneon gas mixture flows through the control gas gap (referred to the gap between the graphite holder and the stainless steel shell) to maintain specified TC readings, ensuring that target fuel temperatures are within the specification defined by program management (Maki, 2009).

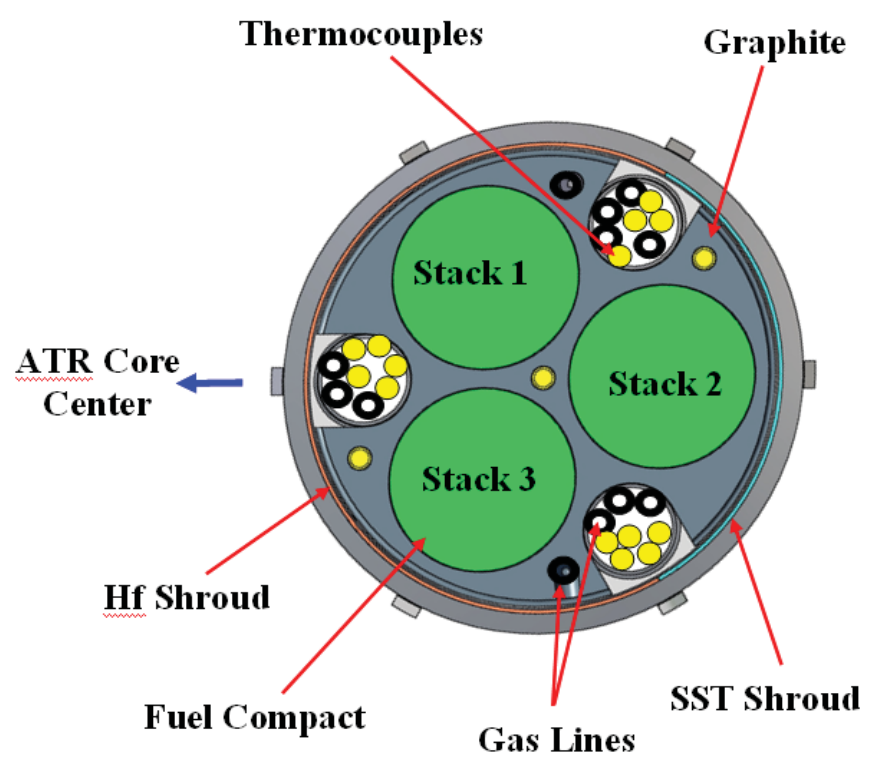

Figure 2. Schematic of a radial cut of an AGR-1 capsule.

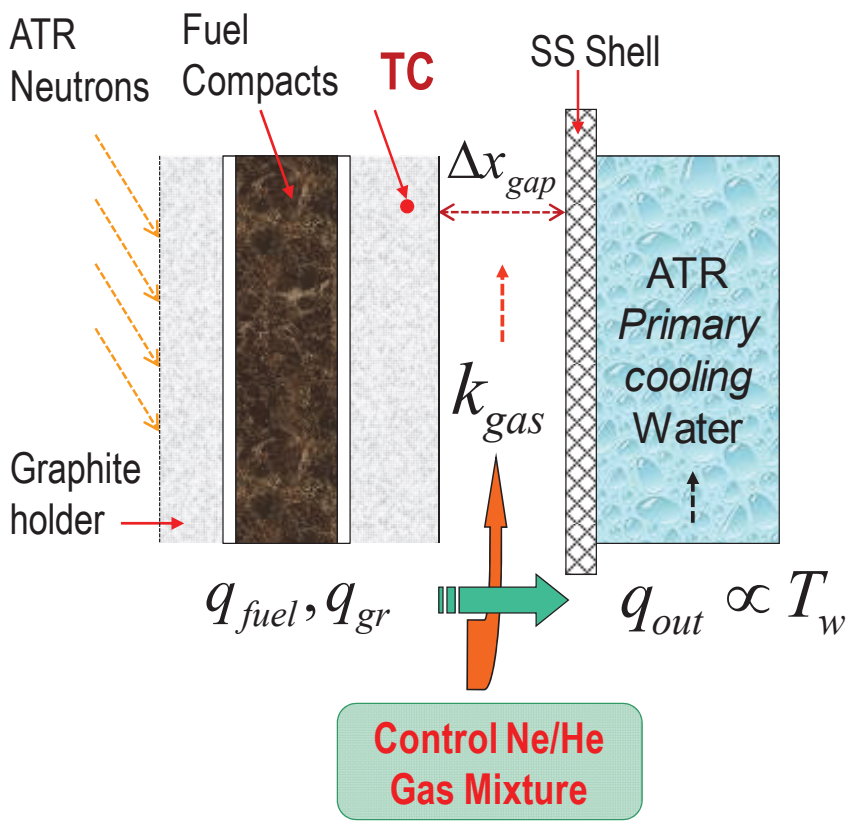

Figure 3. Physical sketch of the axial cut of an AGR-1 capsules. 


\subsection{Thermal Model for AGR-1 Capsules}

ABAQUS-based (Version 6.8-2), finite-element thermal models are created for each capsule of the AGR-1 test to predict daily averages of fuel compact and TC temperatures for the entire irradiation period when the ATR core is at power. The validation of ABAQUS version 6.8-2 was performed and reported in (Hawkes G. , 2012). It comprised of 10 thermal models validating different aspects of ABAQUS' heat transfer abilities. The maximum difference between ABAQUS calculated values and exact theoretical values is just under $2.0 \%$. The governing equation of steady-state conductive heat transfer is expressed as (Hawkes G.L., 2011)

$$
\rho c_{p}\left(u_{x} \frac{\partial T}{\partial x}+u_{y} \frac{\partial T}{\partial y}+u_{z} \frac{\partial T}{\partial z}\right)=\frac{\partial}{\partial x}\left(k(T) \frac{\partial T}{\partial x}\right)+\frac{\partial}{\partial y}\left(k(T) \frac{\partial T}{\partial y}\right)+\frac{\partial}{\partial z}\left(k(T) \frac{\partial T}{\partial z}\right)+Q
$$

where $\rho$ is the density; $c_{p}$ is the specific heat; $u_{x}, u_{y}$, and $u_{z}$ are the three-directional velocities; $T$ is the temperature; $x, y$, and $z$ are direction; $k(T)$ is the thermal conductivity varying with temperature and neutron fluence; and $Q$ is the heat source. The heat transfer through the gas gap is by conduction and radiation only; there is no advection, because of the very low flow rate of gas (30 $\mathrm{cm}^{3} / \mathrm{min}$ ). The governing equation for radiation heat transfer across the control gas gap is

$$
q_{n e t}=\frac{\sigma\left(T_{1}^{4}-T_{2}^{4}\right)}{\frac{\left(1-\varepsilon_{1}\right)}{\varepsilon_{1} A_{1}}+\frac{1}{A_{1} F_{12}}+\frac{\left(1-\varepsilon_{2}\right)}{\varepsilon_{2} A_{2}}}
$$

where $q_{\text {net }}$ is the net heat flux, $\sigma$ is the Stephan Boltzmann constant, $T_{1}$ and $T_{2}$ are the surface temperatures, $\varepsilon_{1}$ and $\varepsilon_{2}$ are the emissivity of Surfaces 1 and 2, $A_{1}$ and $A_{2}$ are the areas of Surfaces 1 and 2 , and $F_{12}$ is the view factor from surface 1 to 2 .

The main time-serried inputs to the model are daily component (namely, fuel compacts and graphite sample holder) heat rates $(Q)$ and neutron fast fluences calculated from the as-run depletion analysis and daily gas compositions of the helium-neon mixture (Ne fraction). The fast neutron fluence is needed for the components' thermal conductivity calculation. The ABAQUS thermal model uses a $\sim 350,000$ eightnode hexahedral brick finite element mesh to estimate capsule temperature profiles as shown in Figure 4 for three fuel stacks. However, uncertainty remains because of a lack of knowledge about phenomena such as heat- and neutron-induced changes in capsule control gas gaps. While no direct measurements of fuel temperature are available, the graphite temperature from TCs in AGR tests was used to validate computer codes.

The PIE metrology data of AGR-1 capsule components indicated that all fuel compacts unevenly shrank and the graphite sample holders unevenly swelled (because of high boron addition to the graphite) for the four middle capsules (2-5), but shrank for Capsules 1 and 6 (with less boron concentration) (Demkowicz, Cole, Ploger, \& Winston, 2011). As a result, the control gap shrank for the four middle capsules and expanded for the top and the bottom capsules; however, the gap between the graphite body and the fuel compact increased, offsetting somewhat the reduction in control gap in calculating fuel temperature. Thus, this behavior is taken into account when calculating the AGR-1 temperatures. The AGR-1 thermal model assumes that the control gap is evenly and linearly reducing for the four middle capsules and slightly increasing for the top and bottom capsules over the entire AGR-1 irradiation from initial actual hot gap size (subtracting thermal expansion of graphite holder) to the estimated end gap. Because there was no fuel particle failure in AGR-1, the R/B from the heavy metal contamination, which is very sensitive to temperature, is thus a measure of the temperature in each capsule. Therefore the end 
gap sizes were estimated by maximizing correlation between the temporal temperature predictions and the temporal $\mathrm{R} / \mathrm{B}$, indicating that the thermal model is capturing all of the important physical phenomena. The reduction of the end gap for the four middle capsules could be as much as $50 \%$ of initial gap.

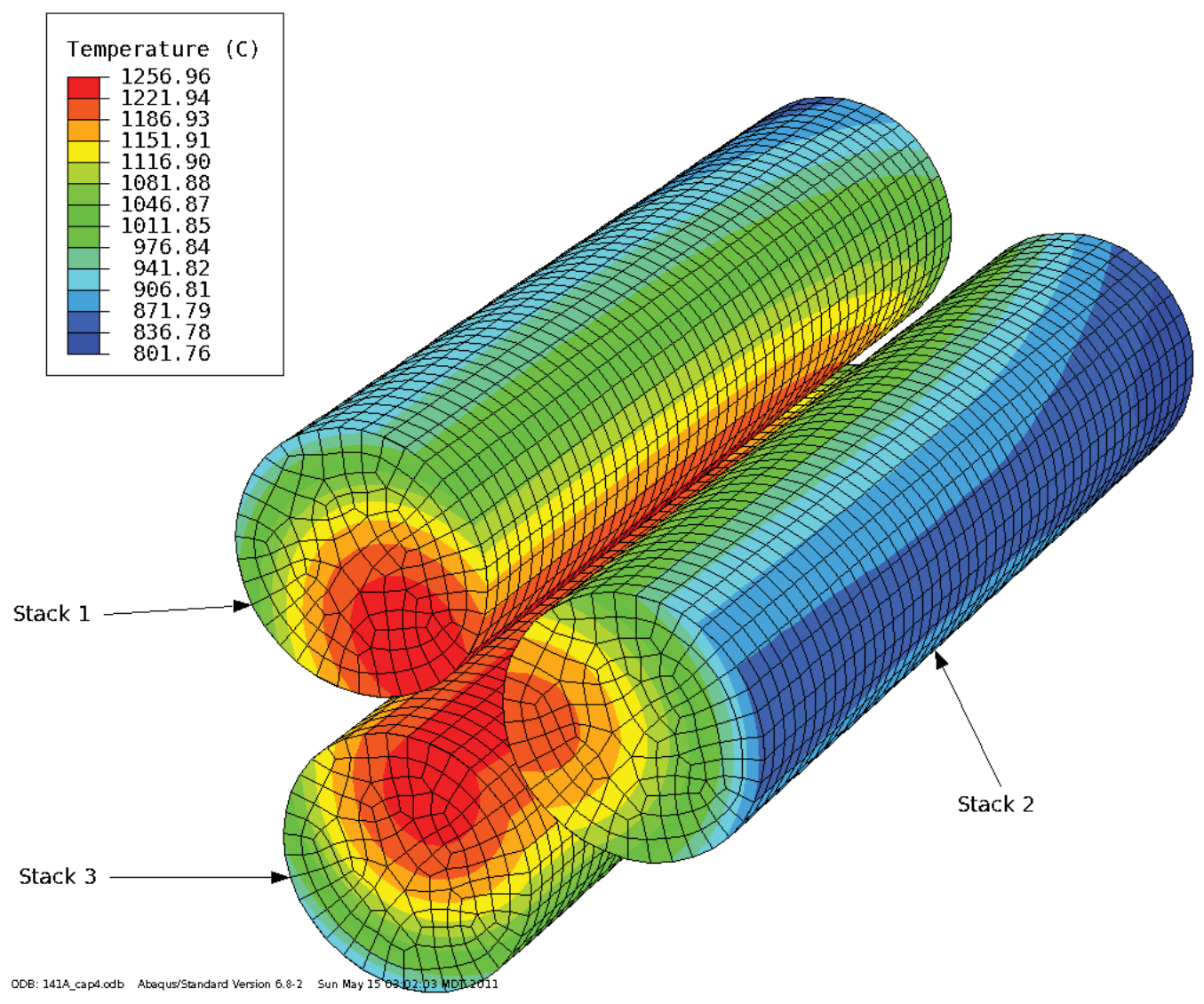

Figure 4. Temperature distribution in cutaway view of three fuel stacks.

Besides the gas gap assumptions, the following factors and model assumptions may also contribute to predicted temperature uncertainties (Hawkes G. , 2012):

1. Heat rates from components (excluding fuel compacts divided into two nodes) and fluences are spatially constant and vary only with time for each capsule.

2. Graphite and compact thermal conductivities varying with fluence and temperature are taken from legacy experiment correlations and scaled for AGR-1 material density.

3. Gas mixture thermal conductivity is determined by kinetic theory of gases using pure gas properties of helium and neon to determine mixture properties.

4. Radiation heat transfer occurs from the graphite holder to the stainless-steel retainer, graphite holder to thru-tubes, and thru-tubes to the stainless-steel retainer. Emissivities of 1.0 and 0.99 were assumed for these surfaces in order to closely match the TC readings during earlier cycles, when TC performance is believed to be more reliable.

5. There is no axial heat conduction from one capsule to the next.

The example of fuel temperature distribution in cutaway view of three fuel stacks presented in Figure 4 , is a typical distribution for all capsules and at other times (Hawkes G. , 2012). Temperatures range from $802^{\circ} \mathrm{C}$ to a maximum of $1256^{\circ} \mathrm{C}$. 


\section{ESTIMATION OF INPUT PARAMETER UNCERTAINTIES AND SENSITIVIES}

\subsection{Approach}

To quantify the uncertainty of AGR calculated temperatures, ABAQUS model input parameters of potential importance are identified. Identification has two parts: (1) using expert judgment, determine parameters with the largest uncertainties and estimate these uncertainties, and (2) using sensitivity analysis, determine parameters that the modeling is most sensitive to, and refine the estimates of these sensitivities.

The parameter uncertainties and sensitivity coefficients are combined and propagated to quantify the overall uncertainty using Eq. 3, because the predicted temperature can be assumed to be the weighted summation of input parameters. This assumption is confirmed during sensitivity analysis.

$$
\sigma_{T}^{2}=\sum_{i}^{n} a_{i}^{2} \sigma_{i}^{2}+\sum_{i}^{n} \sum_{j \neq i}^{n} \rho_{i j} a_{i}^{2} \sigma_{i}^{2} a_{j}^{2} \sigma_{j}^{2}
$$

where: $\sigma_{T}^{2}$ is the overall uncertainty in terms of variance,

$a_{i}^{2}$ is the square of the sensitivity coefficient for parameter $i$,

$\sigma_{i}^{2}$ is the uncertainty of input parameter $i$ in terms of variance,

$\rho_{i j}$ is the correlation coefficient for input parameters $i$ and $j$.

\subsection{Rationale for Basis of Uncertainty Analysis}

The following two sections provide the rationale for basing this uncertainty analysis solely on the propagation of model input parameter uncertainties and sensitivities.

\subsubsection{Improved Agreement between Calculated and Measured TC Readings}

Early analysis of thermocouple data indicated that they performed reliably during the beginning cycles of irradiation. Therefore, the thermal models were calibrated to match the TC readings during this portion of the irradiation. As discussed in Section 1.3 and Section 2.4.1, the updated control gap distance models for all capsules lead to significantly improved fit between calculated and measured TC readings (Pham, Hawkes, \& Einerson, 2012). Figure 5 presents temperature residuals (differences between measured and calculated temperatures) of two peripheral TCs in Capsule 4 (top row) and Capsule 6 (bottom row) for two versions of the AGR thermal model as functions of EFPD. The only difference between the two versions of the thermal model is the gas gap distance model: the first version (run 1) assumes constant gas gap over time and the second version (run 2) assumes linear gap change depending on capsule fluence as shown in eq. 5. The peripheral TCs are used for demonstration of improved fit because temperature at these TCs is most sensitive to variation of the gap distance (subsequently, variation in the gap thermal resistance). In other words, the updated gas gap model has the most effect on predicted temperatures at these TC locations. Capsules 4 and 6 are used because of their stark differences of boron carbide concentration ( $7 \%$ vs. 5.5\%) and location in the test train (middle vs. outer). These differences lead to different directions of the gap change: the gap in Capsule 4 is decreasing significantly (up to $50 \%$ of start gap) because of swelling in the graphite holder while the gap in Capsule 6 is slightly increasing (up to $\sim 10 \%$ ) due to shrinkage in the graphite holder as shown in Figure 12. 
As a result, TC residuals in Capsule 4 (top row in Figure 5) show much improvement in terms of TC fitting for run 2 results (dot symbols show a random pattern around zero for entire AGR-1) relative to run 1 results (triangle symbols show downward trend over time indicating clear departure of TC readings from simulation as the experiment progresses). The continued agreement of these TC readings over the entire AGR-1 suggests negligible model bias of the run 2 results with updated linear gas model. This leads to reduction of overall temperature prediction uncertainty due to elimination of the model bias. The improvement in agreement between measured and calculated TCs in Capsule 6 is much smaller (slightly flatter run $2 \mathrm{TC}$ residuals as shown by the dots in the bottom row in Figure 5) due to much smaller gap change over time.
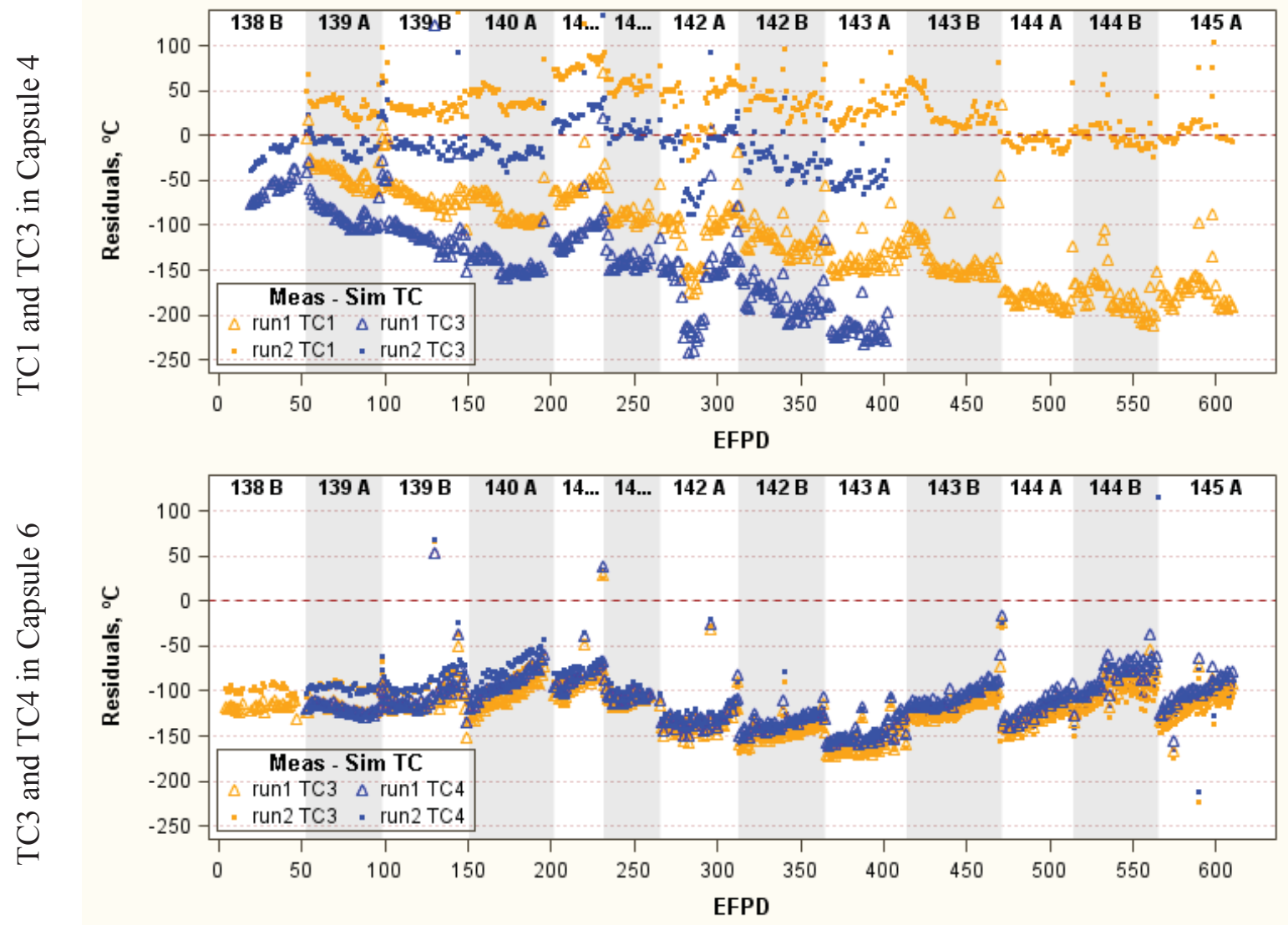

Figure 5. The TC residuals of the original (run 1) and updated (run 2) thermal models for Capsules 4 and 6.

The residuals for operational TCs in the AGR-1 six capsules are plotted in Figure 6. Continued monitoring and analysis of residuals beyond the calibration period show a pattern around and near zero for at least one TC in every capsule except Capsule 6. This suggests negligible model bias and it follows that the uncertainty quantification can be adequately performed relying solely on the model input parameters. For Capsule 6, a bias is present but is largely compensated for by the $10 \%$ bias identified as a portion of the fuel heat rate uncertainty. 


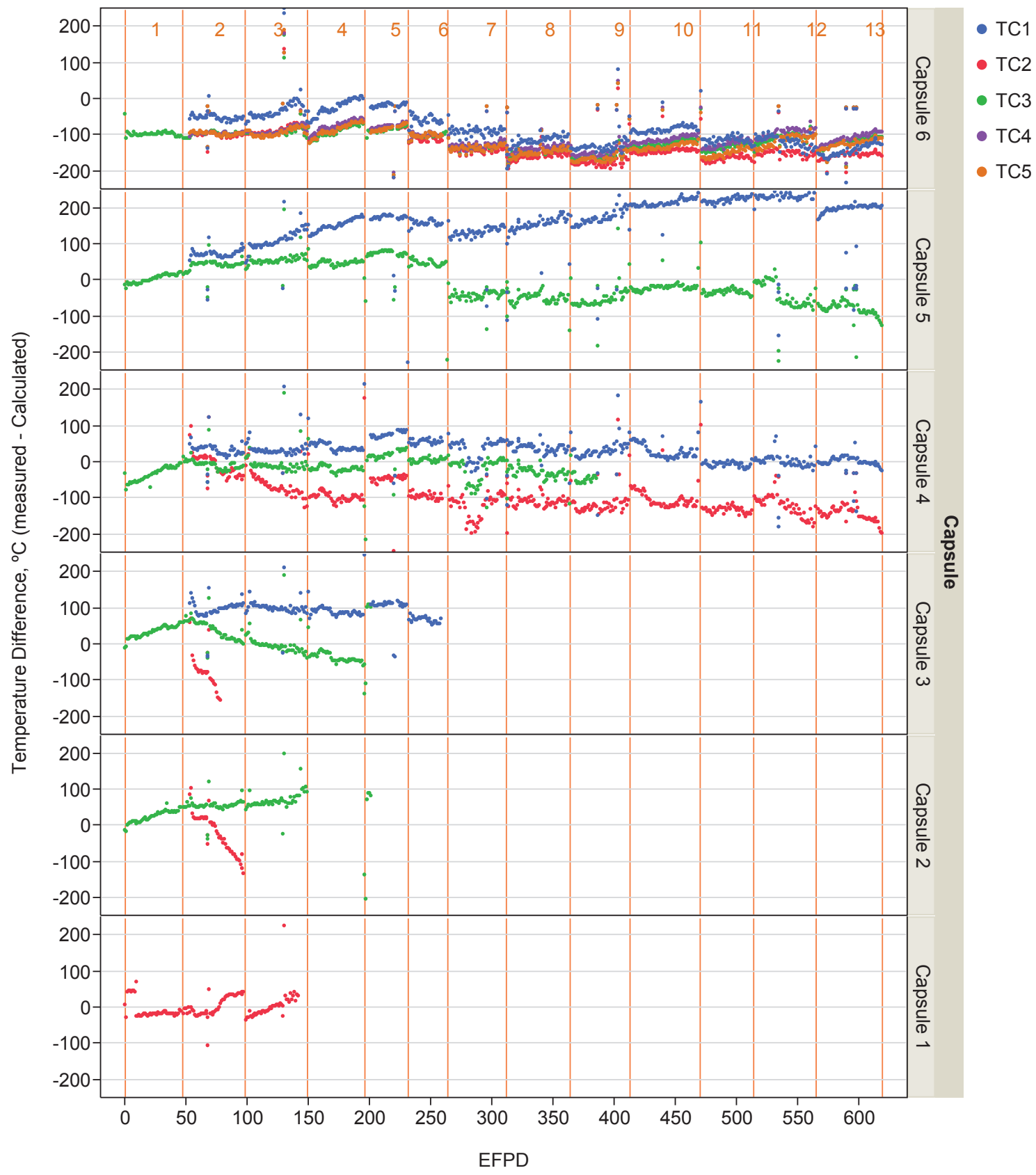

Figure 6. Difference between measured and predicted thermocouple temperatures. 


\subsubsection{Comparison between R/B and Fuel Temperature}

Since there were no particle failures in the six capsules of AGR-1, the fission product R/B ratios are known to be proportionally dependent on fuel temperature. Even though the complex analytical relationship between $\mathrm{R} / \mathrm{B}$ and temperature is not available, the correlation between $\mathrm{R} / \mathrm{B}$ and fuel temperature temporal profiles indicates that the capsule thermal model correctly included important physical phenomena occurring in the capsules. Therefore, comparison between fuel temperature and R/B helps demonstrate that the calculated capsule fuel temperatures correctly reflect the capsule thermal condition. For visual correlation comparison, the capsule daily volume average fuel temperature ( $T_{\text {Nor }_{i}}$ ) and $\log$ of $\mathrm{R} / \mathrm{B}$ for $\mathrm{Kr}-85 \mathrm{~m}$ radionuclide $\left(\log (R / B)_{N_{N o}}\right)$ are linearly normalized as:

$$
T_{\text {Nor }_{i}}=\frac{T_{i}-\left(T_{a v e}-T_{2 \sigma}\right)}{2 T_{2 \sigma}}
$$

where $T_{i}$ is the volume average fuel temperature at time (i), $T_{\text {ave }}$ is the time average volume average fuel temperature at the end of irradiation, and $T_{2 \sigma}$ is two standard deviations. Equation 4 is also used to normalize the $\log (\mathrm{R} / \mathrm{B})$. To increase accuracy of the comparison, the fuel temperature and $\log (\mathrm{R} / \mathrm{B})$ data used include only data during time spans when the ATR core is at full power.

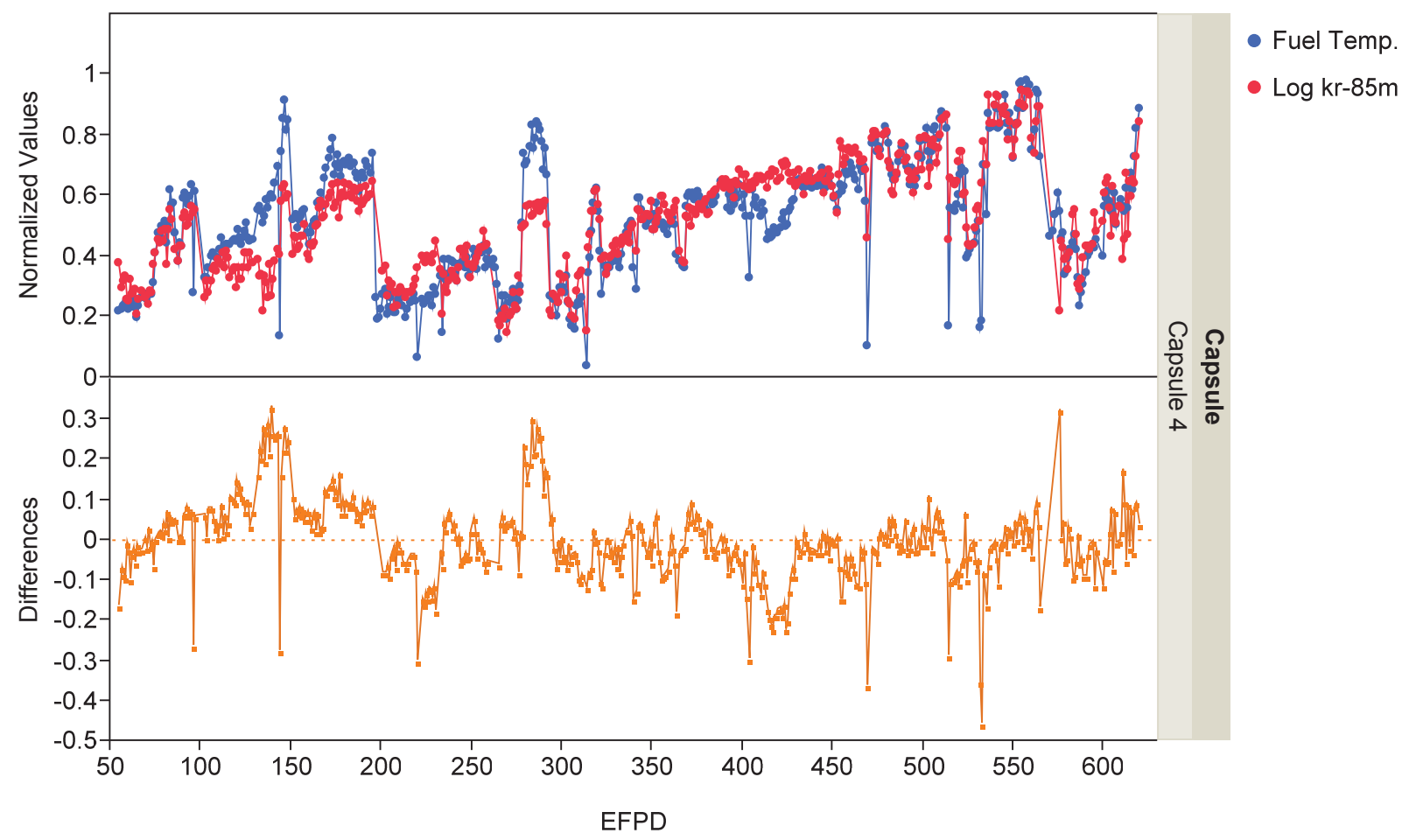

Figure 7. Capsule 4 normalized volume average fuel temperatures, normalized $\log (R / B)$ and their differences.

Figure 8 shows the normalized volume average fuel temperature and normalized fission product $\log (\mathrm{R} / \mathrm{B})$ on the top frame and their differences on the bottom frame as functions of EFPD for Capsule 4. The normalized values of $\log (\mathrm{R} / \mathrm{B})$ (red dots) closely follow the normalized fuel temperatures (blue dots) 
for the entire AGR-1 irradiation. The high pairwise correlation coefficient of 0.86 between normalized fuel temperature and $\log (\mathrm{R} / \mathrm{B})$ also indicates the consistency of predicted and actual fuel temperatures. Additionally, the differences between normalized fuel temperature and $\log (\mathrm{R} / \mathrm{B})$ values are scattered around zero as shown on the bottom frame of Figure 9.

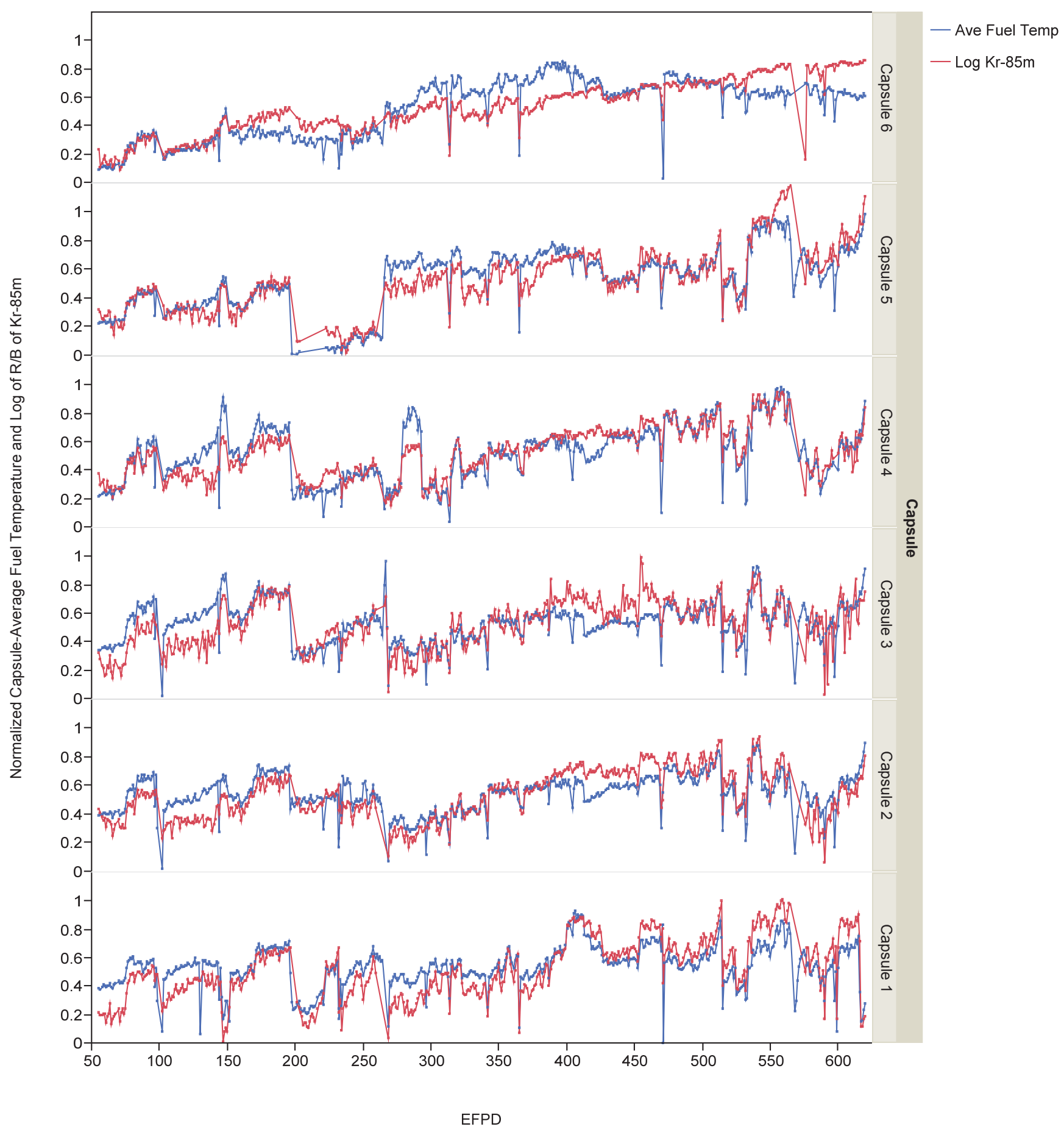

Figure 8. Normalized volume average fuel temperatures and normalized $\log (\mathrm{R} / \mathrm{B})$. 


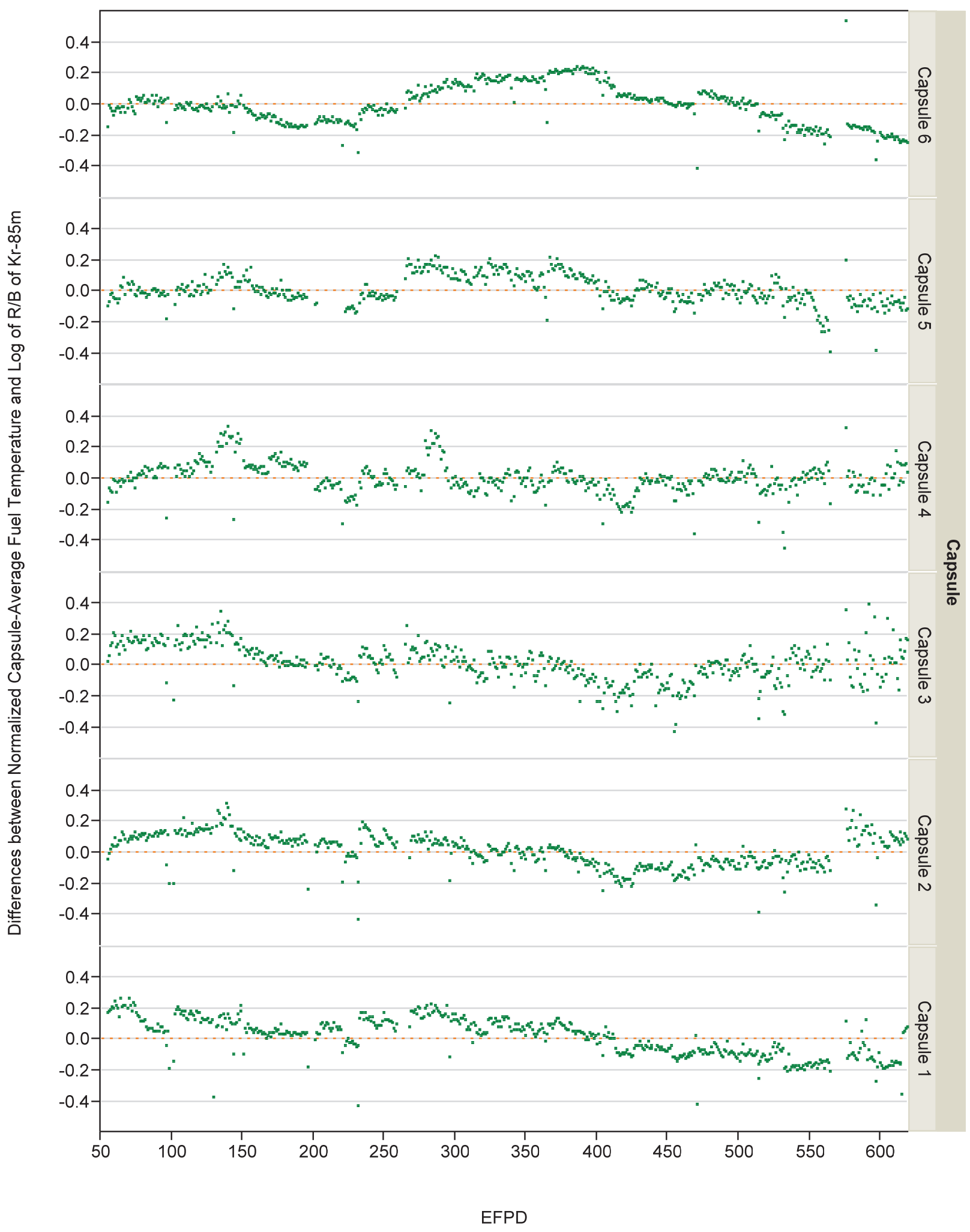

Figure 9. Differences between normalized volume average fuel temperatures and normalized $\log (R / B)$. 
Figure 8 and Figure 9 show the normalized volume average fuel temperatures, normalized $\log (\mathrm{R} / \mathrm{B})$, and their differences for all six capsules. The excellent fit over the entire range of capsule thermal conditions throughout the AGR-1 irradiation for all six capsules provides confidence that the thermal model has appropriately included all important physical phenomena occurring in the capsule. This supports the assumption of negligible model bias and the decision to base the uncertainty analysis solely on model input parameters.

\subsubsection{TC Residual Variation}

The histograms of daily residuals for one operational TC in each capsule, and their statistics (average and standard deviation), are presented in Figure 10. The near zero average in most capsules indicates that the model bias is negligible. The TC residual variation includes variation of both calculated and measured temperatures. The adequacy of basing the uncertainty analysis on model input parameters is confirmed when the TC residual variation is consistent with the model uncertainty. The overall uncertainty results presented in Section 4 show this consistency.

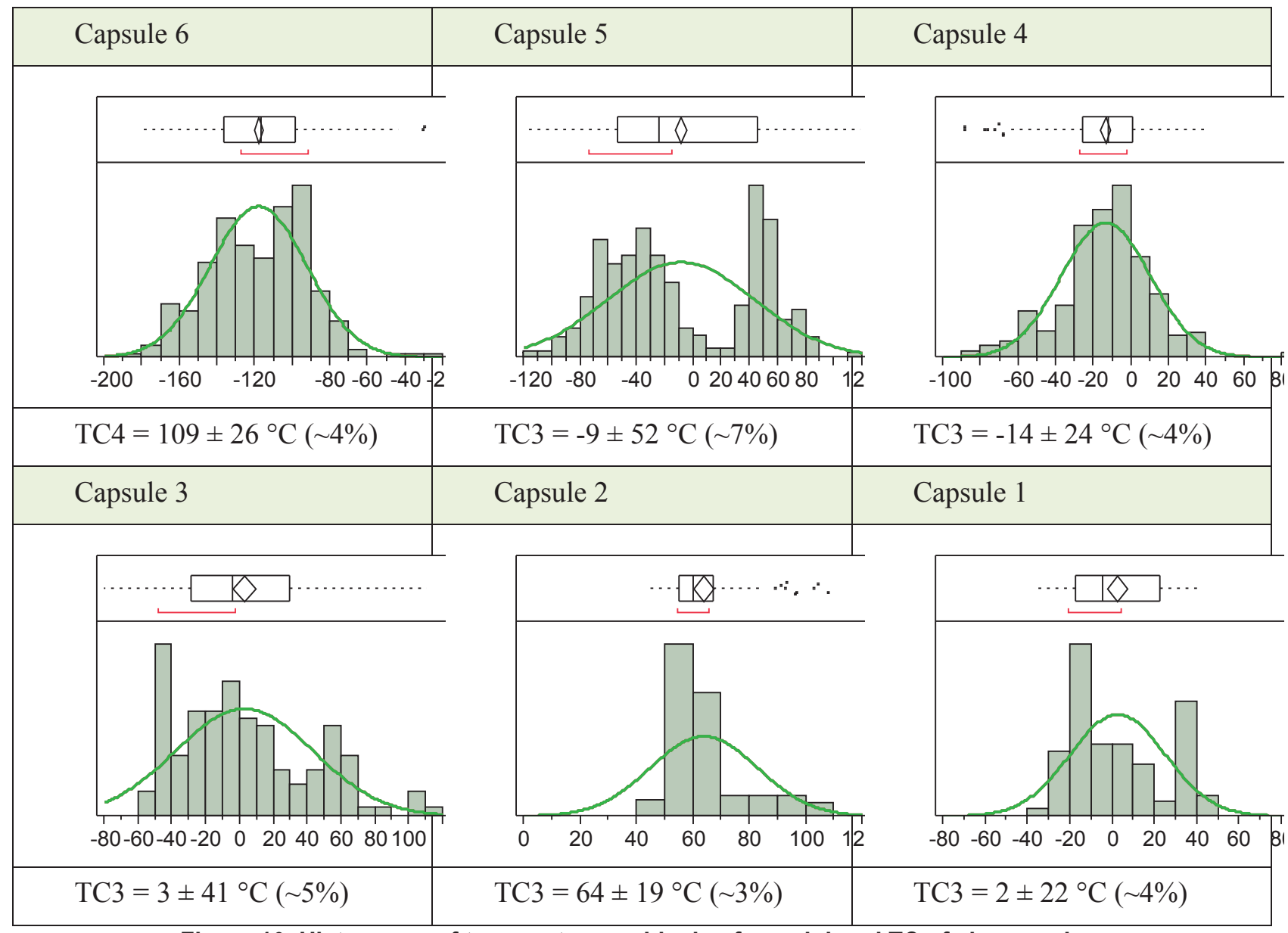

Figure 10. Histograms of temperature residuals of a peripheral TC of six capsules. 


\subsection{Parameter Selection}

The selection of input parameters for uncertainty quantification of the AGR-1 calculated temperatures is based on the ranking of their influences on variation of temperature predictions. The influence ranking is highest for parameters with large uncertainty and/or large sensitivity.

The sensitivity evaluation of the temperature calculations was performed for the AGR-1 fuel experiment on an individual capsule (Capsule 4) by the modeler (Hawkes G.L., 2011). A series of cases was compared to a base case by varying different input parameters to the ABAQUS finite element thermal model. The tornado plot in Figure 11 shows the most sensitive input parameters on peak fuel temperature variations, sorted from largest to smallest, for Capsule 4 during the second AGR-1 cycle (139A) on 3/13/2007. According to this study, the parameters that the model are most sensitive to are control gap distance, heat rate in the fuel, and control gas composition (e.g., neon fraction). The next four are heat rate in the graphite, graphite thermal conductivity, fuel conductivity, and gap conductivity between compact and graphite holder.

Table 1 presents input uncertainties for the five most uncertain input parameters as judged by the NGNP AGR experts (Petti, et al., 2011). Expert judgment takes into account machining tolerances, physics code calculations, mass flow controllers, and engineering experience.

After combining input parameter uncertainties with sensitivities, the five most influential input parameters on AGR-1 calculated temperatures are; control gas gap distance, heat rate in fuel compacts, neon fraction, graphite thermal conductivity, and fuel compact thermal conductivity.

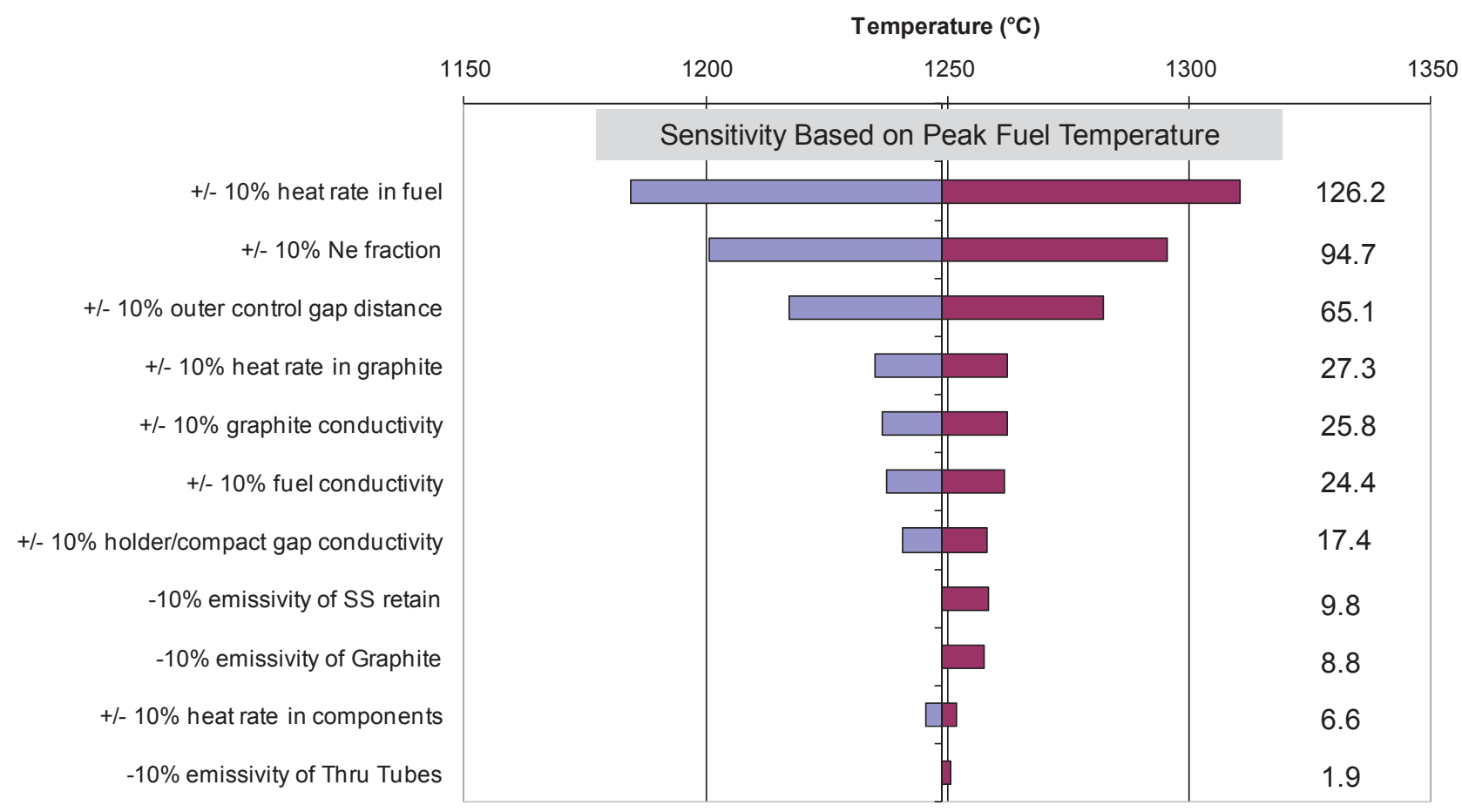

Figure 11. Tornado plot of peak fuel temperature sensitivity. 
Table 1. Uncertainties of the most significant parameters of the AGR-1 thermal model.

\begin{tabular}{|c|c|c|c|}
\hline Input parameter & $\begin{array}{l}\text { Random } \\
(\%)\end{array}$ & $\begin{array}{l}\text { Bias } \\
(\%)\end{array}$ & Comments \\
\hline Control gap distance & $3.5-10.7$ & 25 & $\begin{array}{l}\text { Random: } \sim 1 \text { mil fabricated tolerance. } \\
\text { Bias: time dependent because of shrinking and } \\
\text { swelling. The graphite shrinkage and swelling are } \\
\text { proportional to the reaction rate in the graphite leading } \\
\text { to the physics-based linear gas gap model. The model } \\
\text { is justified by significant correlation between R/B and } \\
\text { fuel temperature profiles. }\end{array}$ \\
\hline Neon fraction & 3 & 0 & Random: $\sim 1 \mathrm{sccm}$ flow rate tolerance. \\
\hline Fuel heat rate & 2.5 & $\begin{array}{c}+10 \text { for } \\
\text { Capsule } 6\end{array}$ & $\begin{array}{l}\text { Random: good fit between predicted and PIE fuel } \\
\text { burnup (less than } 10 \% \text { ) lead to small heat uncertainty. } \\
\text { Bias: ATR axial depletion effects not accounted for in } \\
\text { structure (absorbing) above the core. These affect } \\
\text { mostly Capsule } 6 .\end{array}$ \\
\hline Graphite conductivity & 15 & 0 & $\begin{array}{l}\text { Additional conductivity data for the test graphite } \\
\text { allows a lower uncertainty estimate for graphite than } \\
\text { for fuel. }\end{array}$ \\
\hline Fuel conductivity & 20 & 0 & $\begin{array}{l}\text { Fuel conductivity values used in the model are based } \\
\text { on historic German data corrected for packing fraction } \\
\text { and matrix density. }\end{array}$ \\
\hline
\end{tabular}

\subsection{Input Parameter Uncertainties Based on Expert Judgment}

\subsubsection{Control Gap Distance}

The temperature control gap in a capsule refers to the space between the outer surface of the graphite holder and the inner surface of the hafnium/stainless steel-retainer sleeve.

\subsubsection{Initial Gap Distance}

At the beginning of irradiation the as-fabricated graphite holder outer diameter and capsule retainer sleeve inner diameter are adjusted, taking into account the thermal expansions when capsules are brought up to temperature. The "hot" control gap distance, equal to a half of the difference between the above two adjusted diameters, is used in the ABAQUS model to predict temperatures. They are called initial control gaps and are presented in the second column of Table 2. At this point in time, the uncertainty of control gap distance was based on machining tolerance and engineering experience and assumed to be about onethousandth of an inch (1 mil) for all six capsules. The varied control gap widths lead to different relative uncertainties of the gap as shown in the last column of Table 2 (Hawkes G. , 2011b). The initial (start) gap distances (blue bars) and their error bars of 1 mil presented in Figure 12 for the six AGR-1 capsules show that the top capsule, Capsule 6, has the biggest initial control gas gap followed by the bottom capsule, Capsule 1. The middle capsules, Capsule 3 and 4, have the smallest, therefore they will have the biggest relative initial gap distance uncertainty $(\sim 10 \%)$. 
Table 2. Control gap distance and fabrication uncertainty for AGR-1 capsules.

\begin{tabular}{|ccccc|}
\hline Capsule & Initial Gap (in.) & End Gap (in.) & \% Change & Fabrication Error $(\sigma)$ \\
\hline Capsule 6 & 0.0282 & 0.0308 & $9 \%$ & $3.5 \%$ \\
Capsule 5 & 0.0141 & 0.0061 & $-57 \%$ & $7.1 \%$ \\
Capsule 4 & 0.0100 & 0.0050 & $-50 \%$ & $9.8 \%$ \\
Capsule 3 & 0.0093 & 0.0047 & $-50 \%$ & $10.7 \%$ \\
Capsule 2 & 0.0110 & 0.0040 & $-63 \%$ & $9.1 \%$ \\
Capsule 1 & 0.0180 & 0.0217 & $20 \%$ & $5.5 \%$ \\
\hline
\end{tabular}

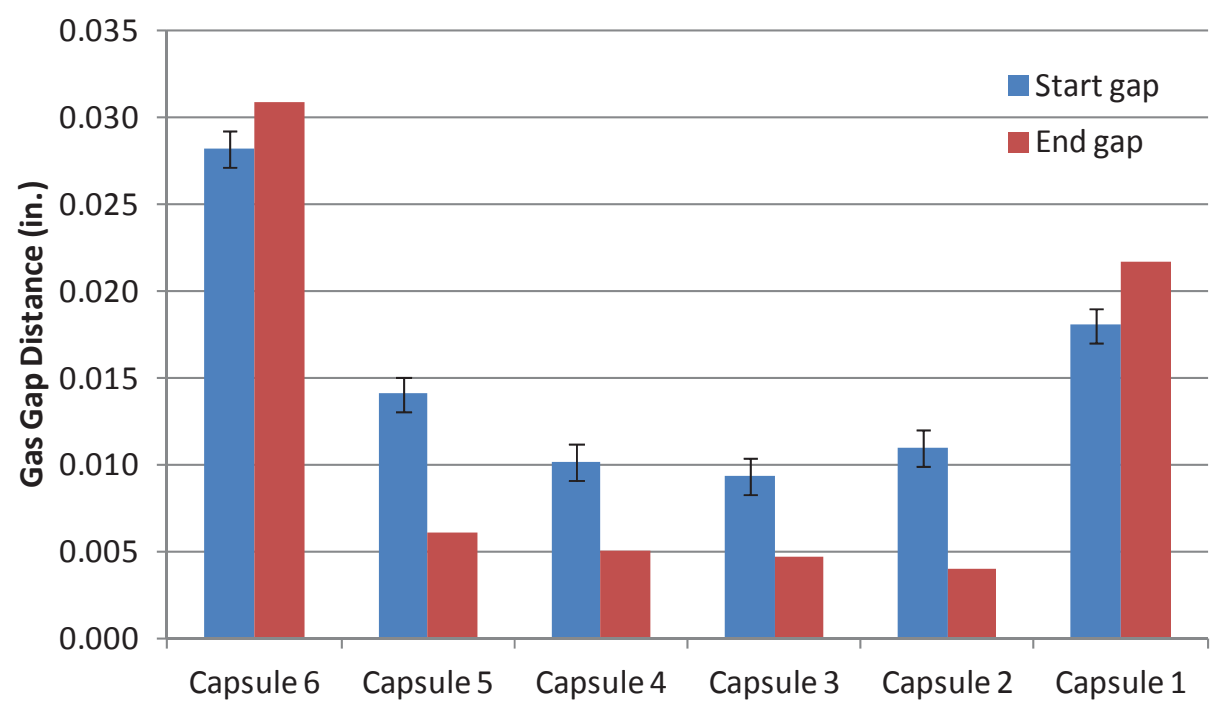

Figure 12. The initial and end gap distance with an error bar of 1 mil for six AGR-1 capsules.

\subsubsection{Gap Distance Changes Over Time}

As the experiment progresses, the material properties of capsule components are changing because of high temperature and neutron fluence. The dimensional measurements of the compacts, graphite holders, and steel capsule shells were performed during PIE and reported in (Demkowicz, Cole, Ploger, \& Winston, 2011). They show significant changes from as-fabricated data. Compact dimensional measurements indicated diametrical shrinkage of 0.9 to $1.4 \%$ and length shrinkage of 0.2 to $1.1 \%$. The amount of shrinkage was somewhat dependent on compact location within each capsule and within the test train. Figure 13 summarizes relative changes in the outer diameter of graphite holders. The diametrical swelling of the holders in Capsules 2-5 (holders initially contained nominally $7.0 \%$ boron carbide as a burnable poison) was 0.7 to $2.1 \%$, and in some cases (particularly in Capsule 3 ) appear to have expanded sufficiently to contact the steel capsule liner in some locations, which complicated extraction from the capsules. By contrast, the graphite holders in Capsules 1 and 6 (containing nominally $5.5 \%$ boron carbide) exhibited diametrical shrinkage of 0.4 to $0.9 \%$. The drastic difference in the dimensional change behavior of the two types of graphite is primarily attributed to the difference in boron carbide $\left(\mathrm{B}_{4} \mathrm{C}\right)$ content and the different location in the test train (outer Capsules 1 and 6 received on average a fast fluence approximately $27 \%$ lower than the other capsules). The Capsule 4 graphite holder was damaged in the disassembly process, so no dimensional data were available for Capsule 4 . As a result, the control gap shrank for the four middle capsules and expanded for the top and bottom capsules. However, the gap between the graphite body and the fuel compacts increases, offsetting somewhat the effect of reduction in the control gap on fuel temperature. 


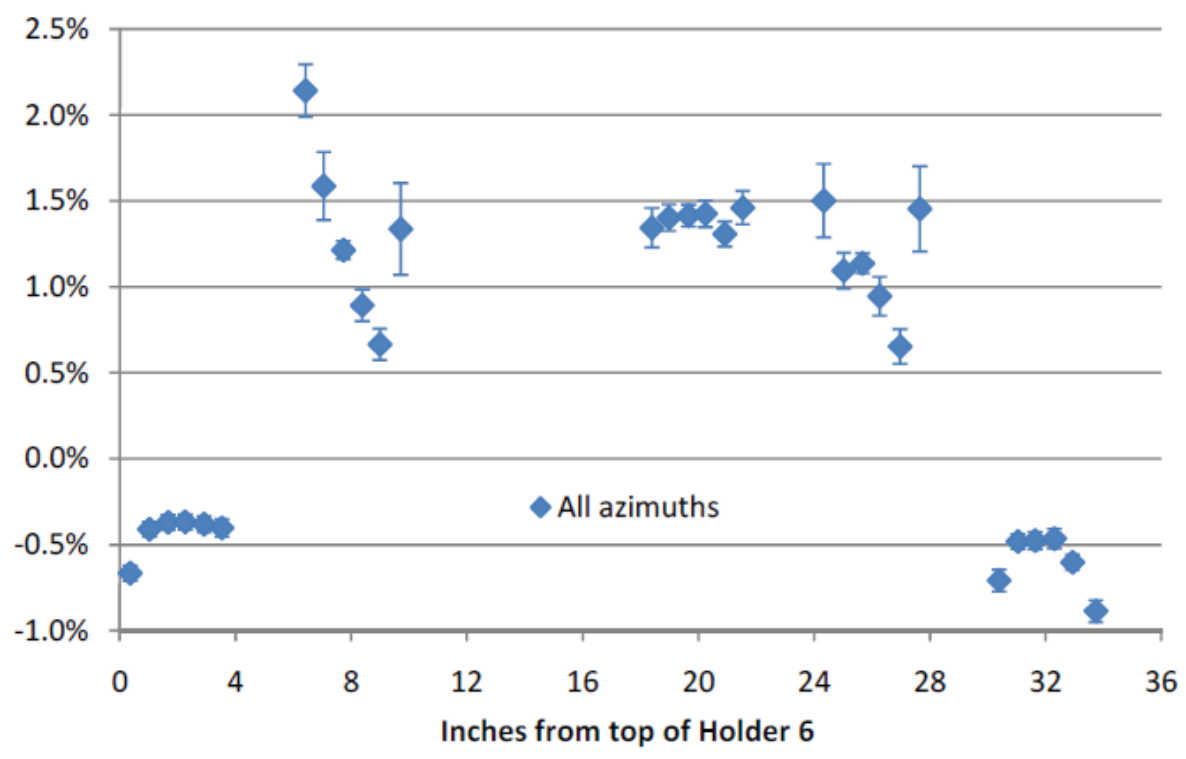

Figure 13. Relative change in outer diameter of graphite holders.

The thermal model for the AGR-1 capsules was modified to account for the control gap changes. First, assumptions are made that the control gap is constant axially and reducing linearly for the middle four capsules, but increasing linearly for the top and bottom capsules over the entire AGR-1 irradiation. This is from the initial gap width ( $\Delta x_{s}$ - Column 2 in Table 2$)$ to the end gap ( $\Delta x_{e}$ - Column 3 in Table 2). Second, the end gap width is estimated by using the TC data and fission product R/B data. This approach is preferred over the use of the PIE measurement data because of the large uncertainty in inferring the actual end gap distance from the cold-state time-delayed PIE of samples apparently contaminated with an unknown substance (gunk) on the capsule shell surface.

Specifically, the end gap width estimation is performed by maximizing the correlation between the predicted temporal fuel temperature and the measured temporal fission product R/Bs (Hawkes G.L., 2011). As a result, the reduction of the end gap for Capsules 2 and 5 is $\sim 60 \%$ of the start gap (Figure 12 and Column 4 in Table 2) and for Capsule 3 is 50\% of the start gap. Since the graphite holder of Capsule 4 was broken during disassembly, the Capsule 4 gap reduction is assumed to be equal to the Capsule 3 reduction of $50 \%$. The gap distance for day 1 is calculated as

$$
\Delta x_{i}=\Delta x_{s}+\left(\Delta x_{e}-\Delta x_{s}\right) * \frac{\text { fluence }_{i}}{\text { fluence }_{A G R-1}}
$$

where $\Delta x_{i}$ and fluence $_{i}$ are gap distance and fluence on day $(i)$, and fluence $e_{A G R-1}$ is the fluence at the end of the AGR-1 test.

The end gap uncertainty is assumed to be equal to 1.5 times the fabrication errors (or initial gap uncertainties) in Table 2.

\subsubsection{Neon Fraction}

Heat produced in the fuel compacts and graphite holder is transferred through the control gas gaps via a gap conductance model using the gap width and the conductivity of the sweep gas (Hawkes G., 2012). The neon fraction of the capsule control gas mixture is, in turn, used in the kinetic theory of gases to determine the gas mixture conductivity. As designed, the capsule gas lines do not cross-talk with each 
other before gas enters an individual capsule and the amount of gas leaking out from a capsule gas line to the leadout is negligible. Therefore, the extra gas leaking in to a capsule is assumed to come only from the leadout flow. As a result, the calculation formula for neon fraction in each capsule, including potential gas leakage both to and from the leadout flow and when the leadout flow contains any amount of helium and neon, is expressed as

$$
F r_{N e}=\frac{Q_{N e}+\max \left(Q_{\text {outlet }}-Q_{N e+H e}, 0\right) * \frac{Q_{\text {Neleadout }}}{Q_{\text {Ne+Heleadout }}}}{\max \left(Q_{\text {outlet }}, Q_{N e+H e}\right)}
$$

where $F r_{N e}$ is the neon fraction and Q is the gas flow in sccm. The neon fraction is 0 for all outage periods. The main source of neon fraction uncertainty is the measurement error in the gas flow meters, which have a $1 \mathrm{sccm}$ tolerance (Table 1) based on engineering assessment. A neon fraction simulation of Eq. 6, with neon and helium flows taken randomly from a normal distribution with the mean value and a standard deviation of $1 \mathrm{sccm}$, was performed for different neon fraction levels. The neon fraction standard deviation is calculated from 100,000 random neon fraction results for each level (mean value). Figure 14 plots the relative neon fraction standard deviations (uncertainty) as a function of the neon fraction values. The power equation option of the trend line feature of the Microsoft Excel platform is used to estimate the function that results in a good fit $\left(\mathrm{R}^{2}=0.9924\right)$. Therefore the relative neon fraction uncertainty can be expressed as a function of neon fraction as

$\sigma_{F r_{n e}}=\frac{2.5487}{F r_{N e}^{1.047}}$

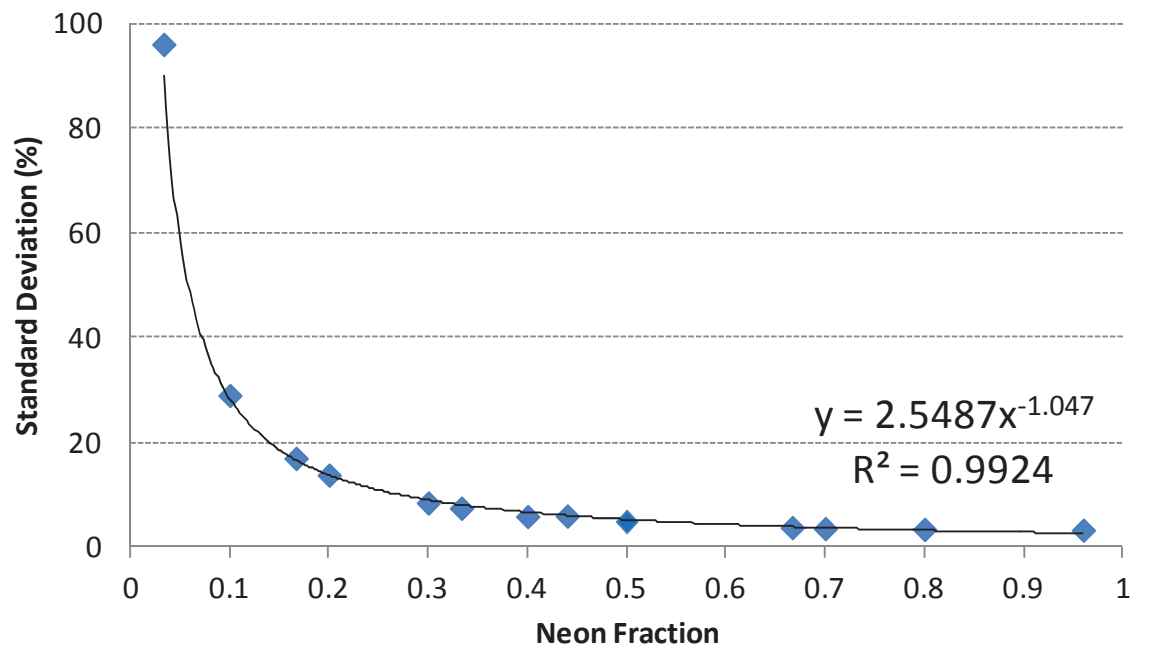

Figure 14. Neon fraction uncertainty and the trend line.

Neon fraction uncertainty is almost the rational function of neon fraction. Thus the relative uncertainty is high at low neon fraction $(\sim 100 \%$ at 0.03 neon fraction) because of high relative uncertainty of the mass flow controllers at lower flow rates. Conversely, the neon fraction uncertainty is zero when the capsule and leadout gas flows are either pure neon $\left(F R_{N e}=1\right)$ or pure helium $\left(F R_{N e}=0\right)$. 


\subsubsection{Fuel Heat Rate}

The fuel heat rates are taken from the as-run physics calculation (Sterbentz J.W., 2011) (Sterbentz, 2011b). Figure 15 shows the smoothed plots of fuel heat rates averaged for each capsule over the time of irradiation. The fuel compacts reach their peak heat rates first for the center capsules $(2,3$, and 4$)$ about $40 \%$ of the way through the irradiation (250 EFPDs). This is because of higher neutron flux at the ATR core center leading to earlier depletion of boron carbide in these capsules. The biggest source of fuel heat rate uncertainty comes from the ATR lobe power calculation and is estimated at $\sim 10 \%$. However, good agreement between burnup calculated by the physics depletion model and PIE measurements, where the difference is less than $10 \%$ for the worst compact, indicates that the instantaneous (daily) fuel fission power uncertainty could be small. The AGR modelers and experts decided that the random portion of the fuel heat rate is $2.5 \%$ for all six AGR-1 capsules and there is a bias of $+10 \%$ of fuel heat rates for Capsule 6. The lack of detail in the physics model about the structure (absorbing of neck shims and regulator rods) above the core is believed to be the cause of the large bias in fuel heat rate for Capsule 6 . This primarily affects just Capsule 6 (top capsule). This fuel power bias in Capsule 6 is also evident in the fact that the calculated temperatures at TC locations are consistently higher than the readings of all five TCs in this capsule.

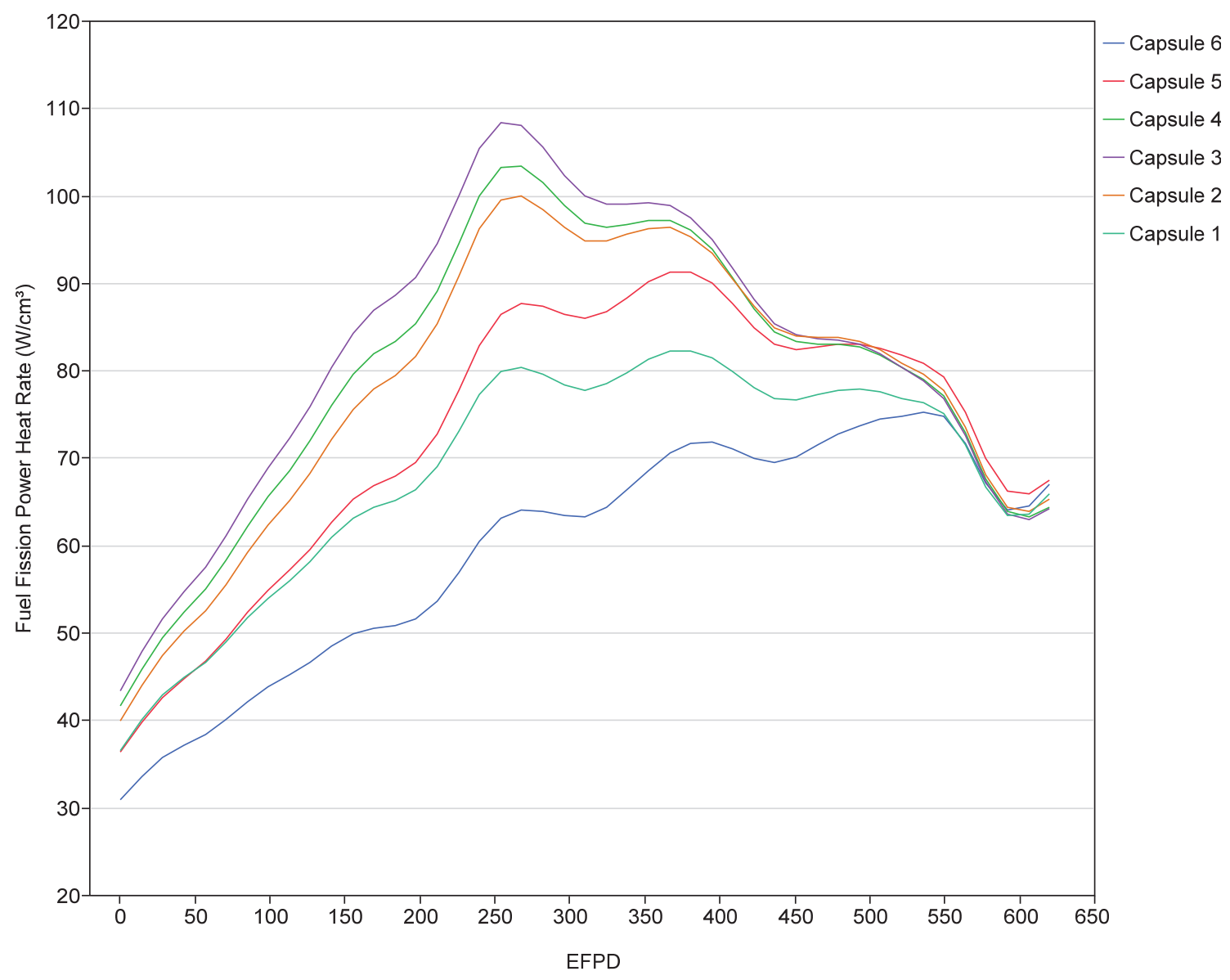

Figure 15. Smooth plots of daily capsule average volumetric heat rates in compacts versus EFPD. 


\subsubsection{Graphite Thermal Conductivities}

Unirradiated graphite thermal conductivity data for the holders were provided by GrafTech as a function of temperature and the weight percent boron carbide present in the material (Snead \& Burchell, 1995). The effect of irradiation on the thermal conductivity of the graphite was accounted for in this analysis using the correlation

$$
\begin{aligned}
& \frac{k_{i r r}}{k_{0}}=\left(0.25-0.00017 * T_{i r r}\right) * A^{*} \log (d p a)+0.000683 * T_{i r r} \\
& A=-1.0
\end{aligned}
$$

where $k_{i r r}$ and $k_{0}$ are thermal conductivities of irradiated and unirradiated graphite, respectively, $T_{i r r}$ is the irradiation temperature $\left({ }^{\circ} \mathrm{C}\right)$, and $d p a$ is displacements per atom. The multiplier used to convert fast fluence $(>0.18 \mathrm{MeV})$ to dpa is $8.23 \times 10^{-26} \mathrm{dpa} /\left(\mathrm{n} / \mathrm{m}^{2}\right)$. Figure 16 shows a three-dimensional (3-D) plot of this ratio $\left(k_{i r r} / k_{o}\right)$ varying with dpa and temperature. The ratio of irradiated to unirradiated thermal conductivity increases with higher temperatures and decreases with higher dpa. These correlations are obtained based on different graphite properties than the graphite employed in the AGR-1 test train. The fact that the thermal conductivity for the actual AGR-1 graphite holder has to be extrapolated from given correlations also leads to higher parameter uncertainty. According to the expert assessment, the existence of one data point for validation of the correlation helps to reduce the graphite thermal conductivity uncertainty from an original value of 20 to $15 \%$ for the entire AGR-1 irradiation as shown in Table 1 .

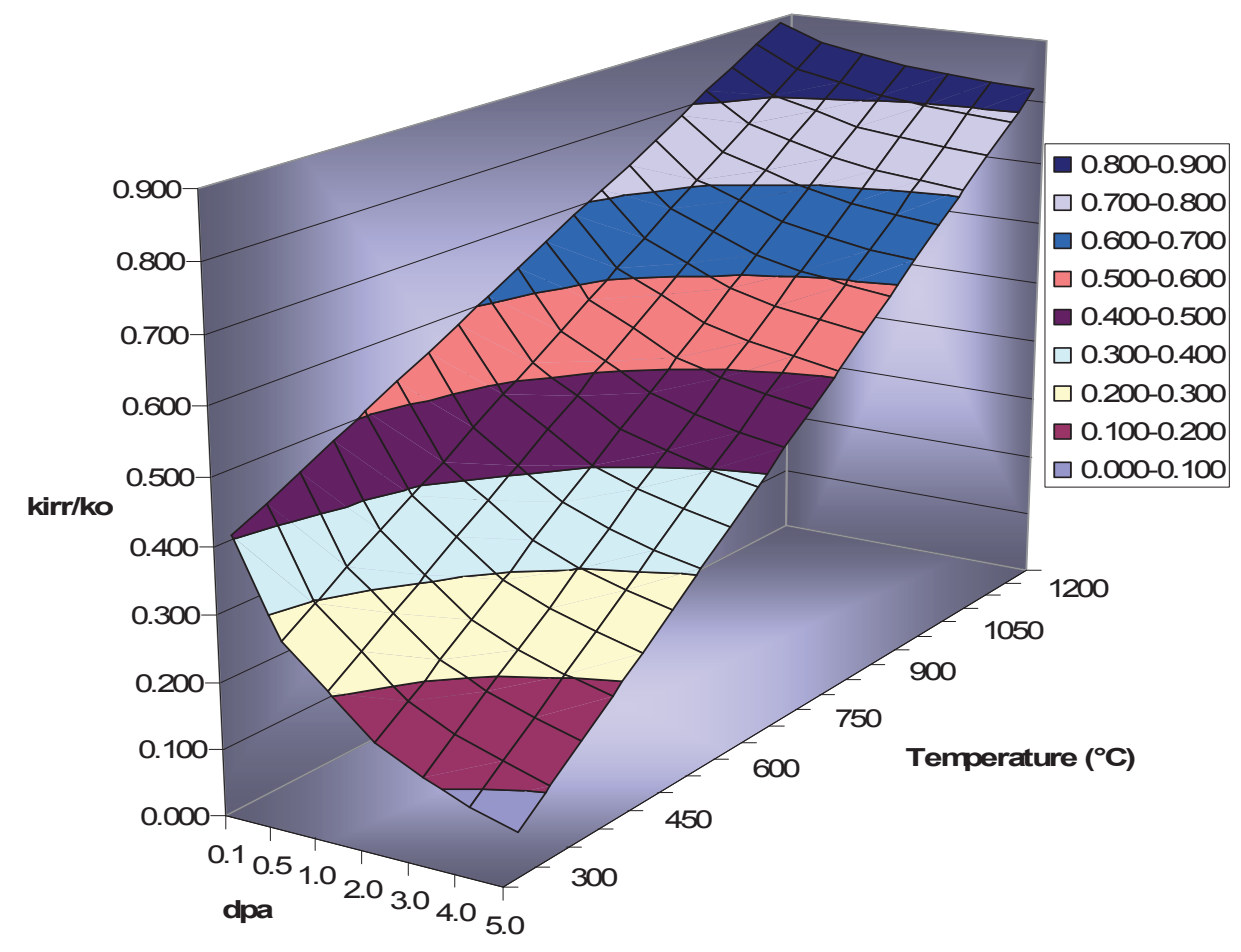

Figure 16. Ratio of irradiated over unirradiated graphite thermal conductivity $\left(\mathbf{k}_{\text {irr }} / \mathbf{k}_{\mathrm{o}}\right)$ varying with temperature and dpa. 


\subsubsection{Fuel Compact Thermal Conductivities}

The fuel compact thermal conductivity was taken from correlations of conductivity with temperature, temperature of heat treatment, neutron fluence, and tristructural-isotropic (TRISO)-coated particle packing fraction (Gontard \& Nabielek, 1990). These correlations were further adjusted to account for differences in fuel compact density. The given correlations were developed for a fuel compact matrix density of $1.75 \mathrm{~g} / \mathrm{cm}^{3}$, whereas the compact matrix used in AGR-1 had a density of approximately 1.3 $\mathrm{g} / \mathrm{cm}^{3}$. Thus the thermal conductivities for AGR-1 compacts were scaled according to the ratio of densities (0.74) in order to correct for this difference. Figure 17 shows a 3-D plot of the fuel compact thermal conductivity varying with fluence and temperature. The lack of experimental data for actual AGR-1 fuel compacts leads to high uncertainty in AGR-1 fuel conductivity, which is estimated to be $20 \%$ for the entire AGR-1 irradiation (Table 1).

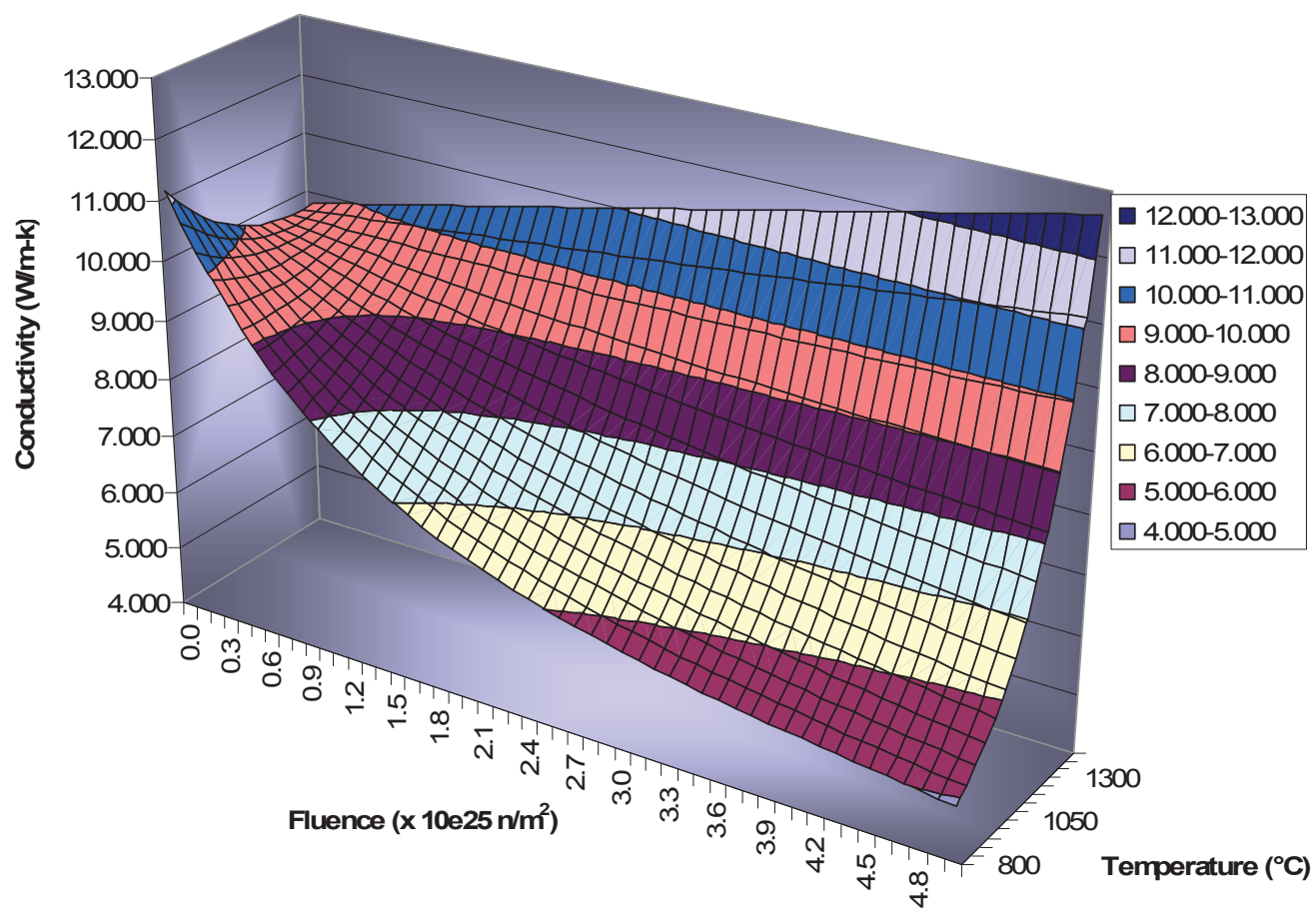

Figure 17. Fuel compact thermal conductivity varying with fluence and temperature.

\subsection{Input Parameter Sensitivity Estimation}

The governing equations for steady-state conduction and radiation heat transfer models (Eq. 1 and 2) used for AGR-1 capsule temperature calculation show complex and nonlinear relationships between output temperature and input parameter over the wide variation of the experiment conditions. This makes it impossible to derive a unique analytical formula to calculate output uncertainty from input variations over the whole AGR-1 experimental condition domain. Additionally, the standard Monte Carlo technique is impractical because of the necessity of requiring hundreds of thousands of simulations to estimate the overall output temperature uncertainty with satisfactory accuracy. The AGR-1 thermal model sensitivity analysis results given in Section 2.3 represent parameter sensitivities within only a small experimental condition domain of $\pm 10 \%$ of the nominal values used in the analysis. Also, the existing sensitivity analysis in (Hawkes G.L., 2011) does not include the investigation of interactive effects of input variables. 
In order to overcome the nonlinearity of the temperature function (e.g., Eq. 1), the AGR-1 thermal condition domain is divided into multiple smaller ranges, within which the output temperature can be estimated as a linear combination of selected input variables. Subsequently, the temperature uncertainty can be calculated from given input uncertainty using standard error propagation of the linear combination (Ostle \& Mensing, 1975).

Table 3 lists four sets of data representing three different thermal condition scenarios in Capsule 4, and one thermal condition scenario in Capsule 6. Capsule 4 has a relatively small initial gas gap and is located in the center of the ATR core where higher exposure of fast neutron fluence leads to a large gas gap reduction by the end of irradiation. Contrarily, Capsule 6 has the biggest initial gas gap and is located at the top of the AGR-1 test train where less exposure to the ATR fast fluence leads to a small gas gap increase. These two capsules are therefore selected to study the effect of location relative to the ATR core on parameter sensitivities.

Table 3. Four selected AGR-1 thermal condition scenarios.

\begin{tabular}{|l|c|c|c|c|c|c|c|c|c|}
\hline Scenario & $\begin{array}{c}\text { Cycle - } \\
\text { EPFD }\end{array}$ & $\begin{array}{c}\text { Gas gap } \\
\text { distance } \\
\text { (in) }\end{array}$ & $\begin{array}{c}\text { Fuel heat } \\
\text { rate } \\
\left(\mathrm{w} / \mathrm{cm}^{3}\right)\end{array}$ & $\begin{array}{c}\text { Fast } \\
\text { fluence } \\
\left(\mathrm{n} / \mathrm{m}^{2} 10^{25}\right)\end{array}$ & $\begin{array}{c}\text { Neon } \\
\text { fraction }\end{array}$ & $\begin{array}{c}\text { Aver. } \\
\text { fuel } \\
\left({ }^{\circ} \mathrm{C}\right)\end{array}$ & $\begin{array}{c}\text { Peak } \\
\text { fuel } \\
\left({ }^{\circ} \mathrm{C}\right)\end{array}$ & $\begin{array}{c}\mathrm{TC} 1 \\
\left({ }^{\circ} \mathrm{C}\right)\end{array}$ & $\begin{array}{c}\mathrm{TC} 2 \\
\left({ }^{\circ} \mathrm{C}\right)\end{array}$ \\
\hline Capsule 4 \\
\hline 1 & $139 \mathrm{~A}-58$ & 0.009532 & 57.15 & 0.3608 & 0.96 & 1038 & 1160 & 808 & 1054 \\
\hline 2 & $142 \mathrm{~A}-303$ & 0.007602 & 111.56 & 1.8658 & 0.49 & 1073 & 1234 & 709 & 1168 \\
\hline 3 & $145 \mathrm{~A}-601$ & 0.005041 & 72.39 & 3.8581 & 1 & 1169 & 1280 & 699 & 1111 \\
\hline Capsule 6 \\
\hline 4
\end{tabular}

\subsubsection{Statistical Experimental Design for Simulations}

In order to be computationally efficient, the statistical experimental design was used to develop the set of simulation runs necessary to estimate all main effects and pairwise interactions of the five important input variables (Ostle \& Mensing, 1975). Table 4 lists the required 51 runs of the ABAQUS code to be made for each thermal condition scenario in Table 3. Note that the number of runs needed for Scenario 3 is reduced from 51 to 42 runs because its nominal neon fraction of 1 will lead to the "high" value of neon fraction being greater than 1 , which is not physically possible. 
Table 4. Experimental design matrix for AGR-1 thermal model sensitivity analysis. ${ }^{a}$

\begin{tabular}{|c|c|c|c|c|c|c|}
\hline Run & Heat Rate & Gas Gap & Ne Fraction ${ }^{\mathrm{b}}$ & Graphite Conductivity & $\begin{array}{c}\text { Fuel compact } \\
\text { conductivity }\end{array}$ & Purpose \\
\hline 0 & Nominal & Nominal & Nominal & Nominal & Nominal & Base line \\
\hline 1 & Low & Nominal & Nominal & Nominal & Nominal & \multirow{2}{*}{ Estimate effect of heat rate } \\
\hline 2 & High & Nominal & Nominal & Nominal & Nominal & \\
\hline 3 & Nominal & Low & Nominal & Nominal & Nominal & \multirow{2}{*}{ Estimate effect of gas gap } \\
\hline 4 & Nominal & High & Nominal & Nominal & Nominal & \\
\hline 5 & Nominal & Nominal & Low & Nominal & Nominal & \multirow{2}{*}{ Estimate effect of $\mathrm{Ne}$ fraction } \\
\hline 6 & Nominal & Nominal & High & Nominal & Nominal & \\
\hline 7 & Nominal & Nominal & Nominal & Low & Nominal & \multirow{2}{*}{ Estimate effect of graphite conductivity } \\
\hline 8 & Nominal & Nominal & Nominal & High & Nominal & \\
\hline 9 & Nominal & Nominal & Nominal & Nominal & Low & \multirow{2}{*}{$\begin{array}{l}\text { Estimate effect of fuel compact } \\
\text { conductivity }\end{array}$} \\
\hline 10 & Nominal & Nominal & Nominal & Nominal & High & \\
\hline 11 & Low & Low & Nominal & Nominal & Nominal & \multirow{4}{*}{$\begin{array}{l}\text { Estimate interactive effect of heat rate } \\
\text { by gas gap }\end{array}$} \\
\hline 12 & Low & High & Nominal & Nominal & Nominal & \\
\hline 13 & High & Low & Nominal & Nominal & Nominal & \\
\hline 14 & High & High & Nominal & Nominal & Nominal & \\
\hline 15 & Low & Nominal & Low & Nominal & Nominal & \multirow{4}{*}{$\begin{array}{l}\text { Estimate interactive effect of heat rate } \\
\text { by Ne fraction }\end{array}$} \\
\hline 16 & Low & Nominal & High & Nominal & Nominal & \\
\hline 17 & High & Nominal & Low & Nominal & Nominal & \\
\hline 18 & High & Nominal & High & Nominal & Nominal & \\
\hline 19 & Nominal & Low & Low & Nominal & Nominal & \multirow{4}{*}{$\begin{array}{l}\text { Estimate interactive effect of gas gap } \\
\text { by Ne fraction }\end{array}$} \\
\hline 20 & Nominal & Low & High & Nominal & Nominal & \\
\hline 21 & Nominal & High & Low & Nominal & Nominal & \\
\hline 22 & Nominal & High & High & Nominal & Nominal & \\
\hline 23 & Low & Nominal & Nominal & Low & Nominal & \multirow{4}{*}{$\begin{array}{l}\text { Estimate interactive effect of heat rate } \\
\text { by graphite conductivity }\end{array}$} \\
\hline 24 & Low & Nominal & Nominal & High & Nominal & \\
\hline 25 & High & Nominal & Nominal & Low & Nominal & \\
\hline 26 & High & Nominal & Nominal & High & Nominal & \\
\hline 27 & Nominal & Nominal & Low & Low & Nominal & \multirow{4}{*}{$\begin{array}{l}\text { Estimate interactive effect of graphite } \\
\text { conductivity by Ne fraction }\end{array}$} \\
\hline 28 & Nominal & Nominal & High & Low & Nominal & \\
\hline 29 & Nominal & Nominal & Low & High & Nominal & \\
\hline 30 & Nominal & Nominal & High & High & Nominal & \\
\hline 31 & Nominal & Low & Nominal & Low & Nominal & \multirow{4}{*}{$\begin{array}{l}\text { Estimate interactive effect of gas gap } \\
\text { by graphite conductivity }\end{array}$} \\
\hline 32 & Nominal & Low & Nominal & High & Nominal & \\
\hline 33 & Nominal & High & Nominal & Low & Nominal & \\
\hline 34 & Nominal & High & Nominal & High & Nominal & \\
\hline 35 & Low & Nominal & Nominal & Nominal & Low & \multirow{4}{*}{$\begin{array}{l}\text { Estimate interactive effect of heat rate } \\
\text { by fuel conductivity }\end{array}$} \\
\hline 36 & Low & Nominal & Nominal & Nominal & High & \\
\hline 37 & High & Nominal & Nominal & Nominal & Low & \\
\hline 38 & High & Nominal & Nominal & Nominal & High & \\
\hline 39 & Nominal & Low & Nominal & Nominal & Low & \multirow{4}{*}{$\begin{array}{l}\text { Estimate interactive effect of heat rate } \\
\text { by fuel conductivity }\end{array}$} \\
\hline 40 & Nominal & Low & Nominal & Nominal & High & \\
\hline 41 & Nominal & High & Nominal & Nominal & Low & \\
\hline 42 & Nominal & High & Nominal & Nominal & High & \\
\hline 43 & Nominal & Nominal & Low & Nominal & Low & \multirow{4}{*}{$\begin{array}{l}\text { Estimate interactive effect of heat rate } \\
\text { by fuel conductivity }\end{array}$} \\
\hline 44 & Nominal & Nominal & Low & Nominal & High & \\
\hline 45 & Nominal & Nominal & High & Nominal & Low & \\
\hline 46 & Nominal & Nominal & High & Nominal & High & \\
\hline 47 & Nominal & Nominal & Nominal & Low & Low & \multirow{4}{*}{$\begin{array}{l}\text { Estimate interactive effect of heat rate } \\
\text { by fuel conductivity }\end{array}$} \\
\hline 48 & Nominal & Nominal & Nominal & Low & High & \\
\hline 49 & Nominal & Nominal & Nominal & High & Low & \\
\hline 50 & Nominal & Nominal & Nominal & High & High & \\
\hline
\end{tabular}




\subsubsection{Statistical Analysis of ABAQUS Runs}

From the ABAQUS output, multiple daily temperatures are obtained such as capsule average, capsule peak temperature, and temperatures at TC locations. For sensitivity analysis of the input parameters, the JMP module (JMP 8.0.2) of SAS (SAS 2009) is used to build a response surface for each of the temperature measures to determine which input terms have significant impacts. The significant coefficients are treated as sensitivities that estimate the rate of change of the temperature with regard to the input and are used to propagate the parameter uncertainty to the output temperatures.

Five parameters are included in this sensitivity analysis, namely the fuel heat rate (HR), the size of the gas gap (GG) between the capsule graphite holder and the stainless steel test train wall, the neon fraction $(\mathrm{NeF})$ in the control gas, graphite conductivity (GC), and fuel compact conductivity (FC). Since the experimental design provides estimation of main effects and pairwise interactions among input variables, the following response surface model containing 20 terms is constructed and studied in the JMP platform for each of five temperature response measures:

$$
\begin{aligned}
f_{T}= & a_{0}+a_{1} H R+a_{2} G G+a_{3} N e f+a_{4} G C+a_{5} F C+a_{6} H R^{2}+a_{7} G G^{2}+a_{8} N e f^{2}+a_{9} G C^{2} \\
& +a_{10} F C^{2}+a_{11} H R \cdot G G+a_{12} H R \cdot N e f+a_{13} H R \cdot G C+a_{14} H R \cdot F C+a_{15} G G \cdot N e f \\
& +a_{16} G G \cdot G C+a_{17} G G \cdot F C+a_{18} N e f \cdot G C+a_{19} N e f \cdot F C+a_{20} G C \cdot F C
\end{aligned}
$$

Because not all nominal values are known, both input variables and output responses are transformed to a relative value ( 0.9 is $10 \%$ less, 1.0 is nominal, and 1.1 is $10 \%$ more) by dividing by the nominal values prior to response surface model fitting.

Regression analysis results of the volume average (VA) fuel temperature (FT) in Capsule 4 are discussed in detail here and complete results are summarized at the end of the section. Figure 18 presents the actual versus predicted plots for VA FT and shows an excellent fit by the model. The analysis of variance results indicate that all coefficients in the model are significantly different from zero, but the five main effects dominate and $\mathrm{Ne}$ fraction squared is most significant of the second order terms. 


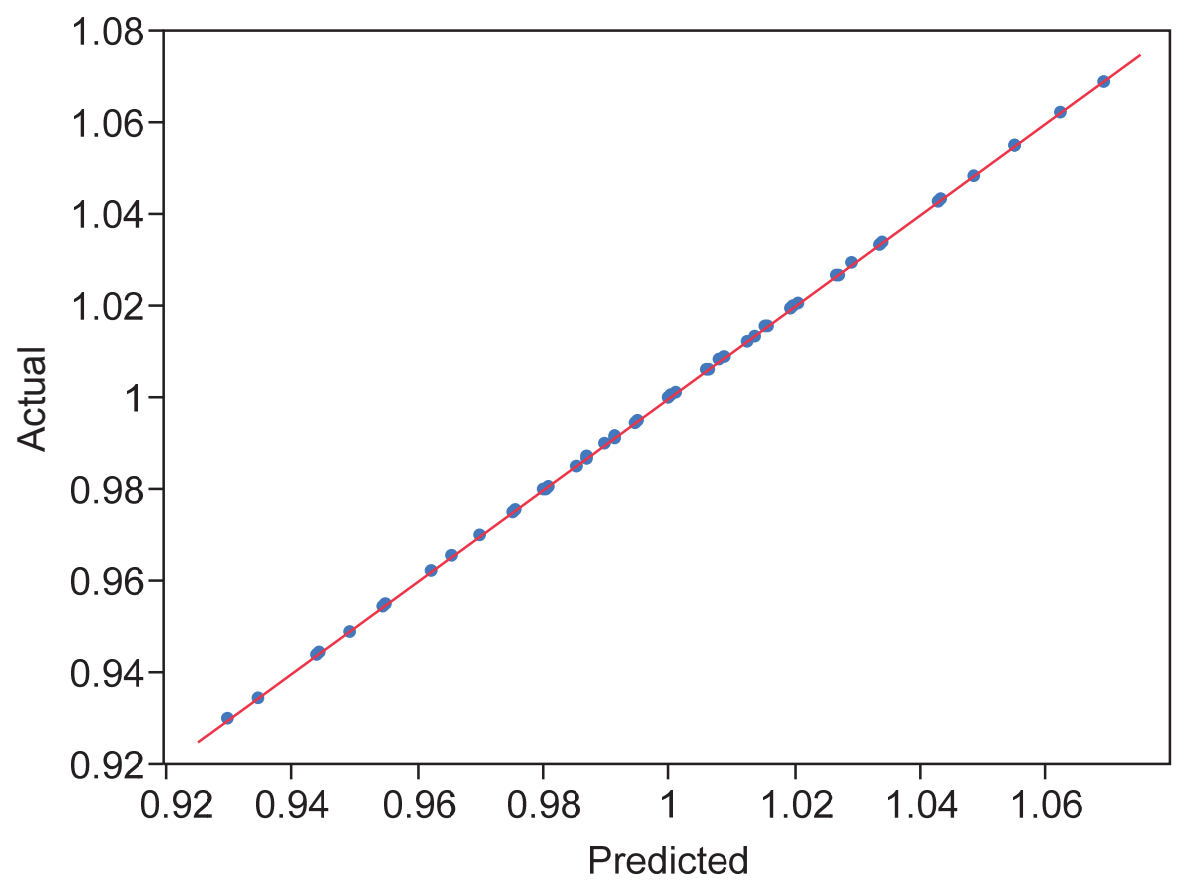

Figure 18. Actual by predicted plot for VA FT for Capsule 4

Figure 19 shows the leverage plots for the five main effects (variables) and neon fraction squared for VA FT. The significant slopes of fuel heat rate, gas gap, and neon fraction indicate that they have the most influence on capsule temperature variations. Even though the estimate of the neon fraction squared term is significantly different from zero, its contribution to fuel temperature variation is small, as shown by the almost horizontal line in the bottom right leverage plot. All other second-order terms in the regression model (Eq. 9) have even smaller contributions to the capsule temperature variations.

The prediction profilers in the JMP "fit model" platform display profile traces for each independent variable and are shown in Figure 20 for fuel and TC temperatures. A profile trace is the predicted response as one variable is changed while the others are held constant at their current values. The absence of curvature in prediction profiles for all variables confirms the linear relationship between response measures and input variables within $10 \%$ of input variations. 

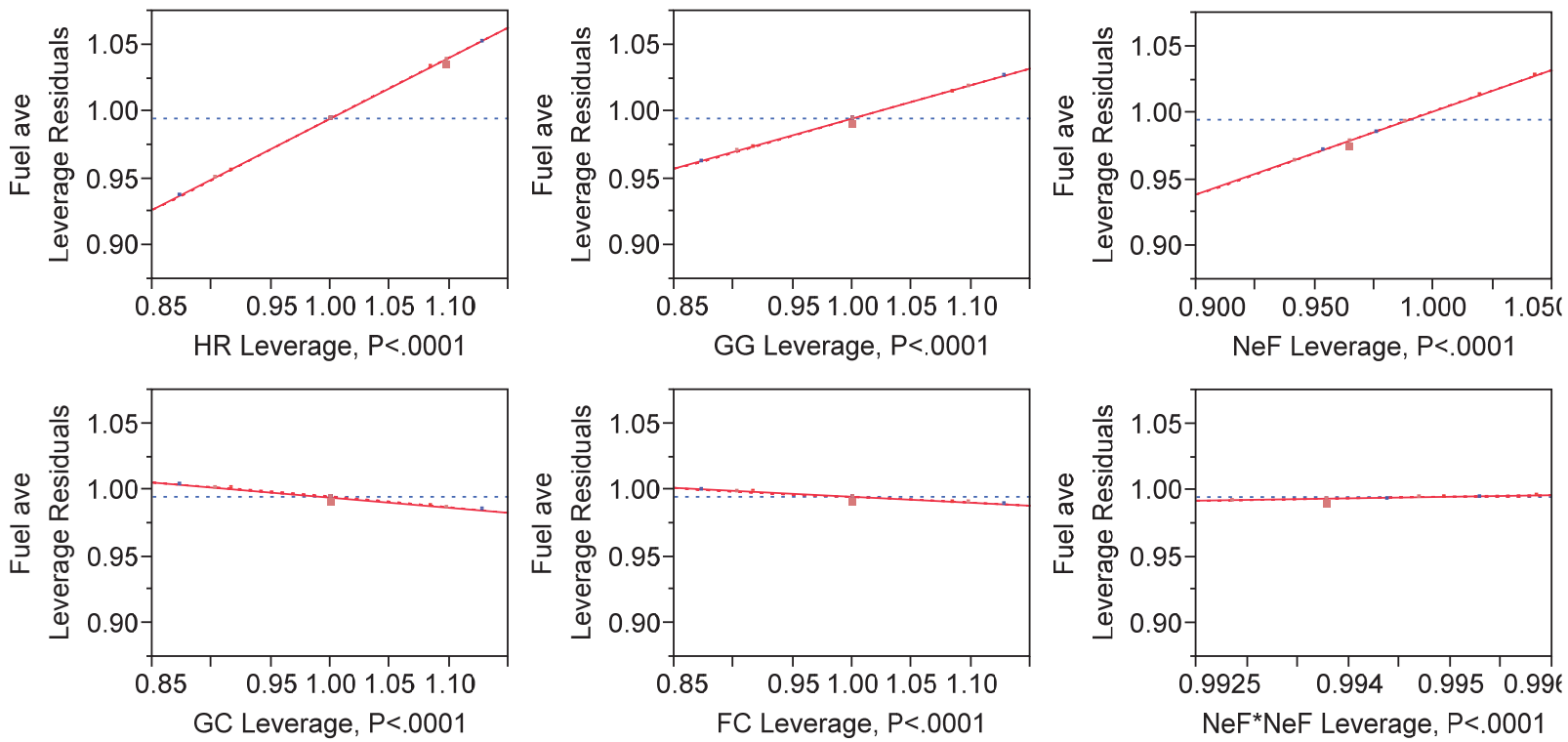

Figure 19. Leverage plots for main effects and the square term of Ne fraction for VA FT.

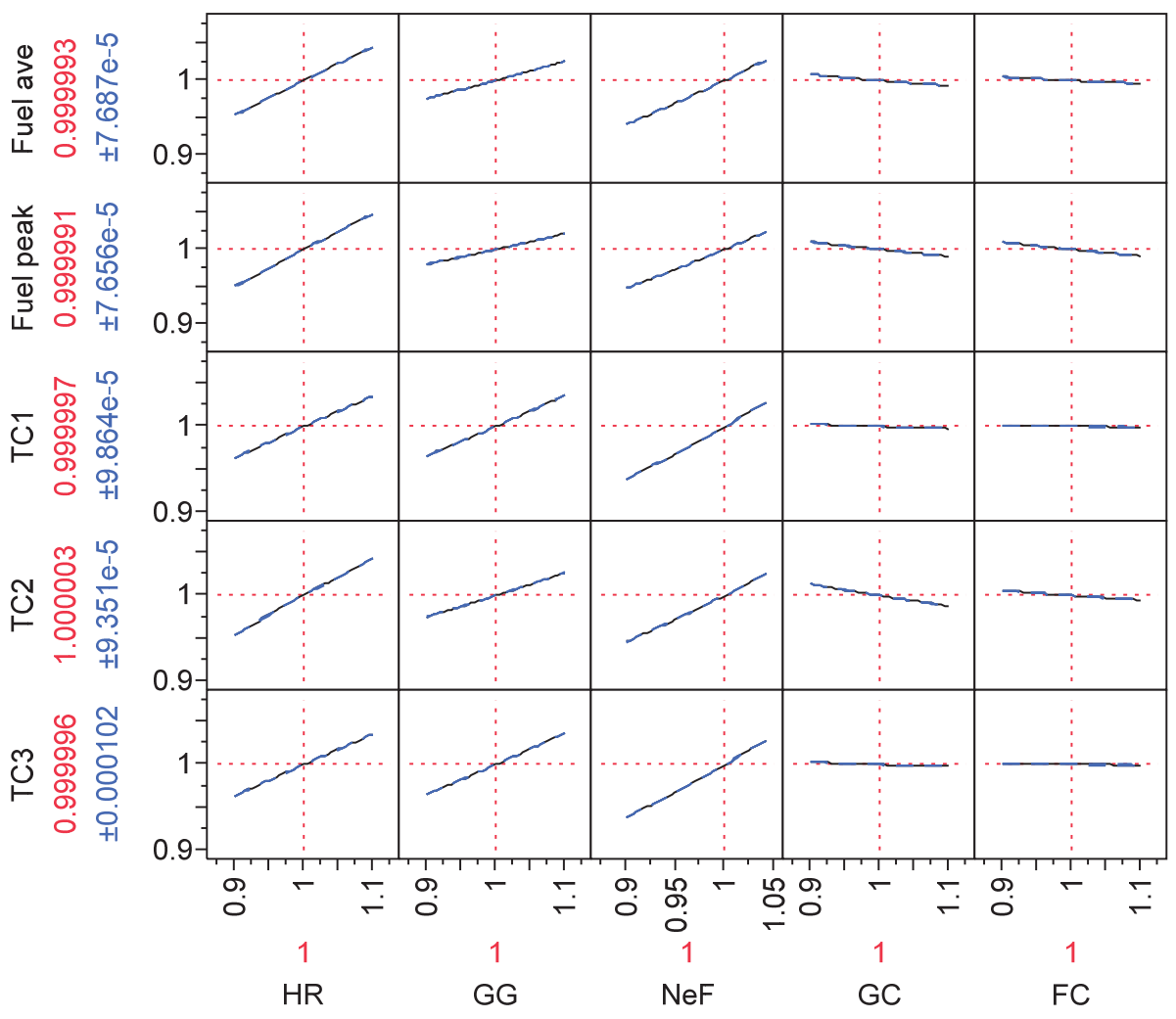

Figure 20. Prediction profiles of functions for fuel and TC temperatures. 
The results presented in Figure 18 to Figure 20 allow for exclusion of all second order terms (square terms of five variables and their pairwise interactions) in the regression model (Eq. 9) because of their relatively negligible contributions to temperature variation. Therefore, the regression model of Eq. 9 can be reduced to a linearized approximation of model temperature as

$$
f_{T}=a_{0}+a_{1} H R+a_{2} G G+a_{3} N e f+a_{4} G C+a_{5} F C
$$

The model coefficient estimates $\left(\mathrm{a}_{0}-\mathrm{a}_{5}\right)$ for VA FT, peak FT, and temperatures at three TC locations for the four scenarios (Table 3) are presented in Table 5. The bar plots in Figure 21 show the variation of parameter sensitivities over different thermal conditions of the three selected scenarios for Capsule 4. This reflects the nonlinear relationship between temperature and the thermal model inputs over the wide thermal condition range of the entire AGR-1 irradiation. Therefore, the model input sensitivities for other time steps will be estimated by interpolation from sensitivities of these four data sets depending on their actual thermal condition input parameters.

Table 5. Parameter estimates for VA FT, peak FT and TC temperatures.

\begin{tabular}{|c|c|c|c|c|c|c|c|}
\hline Response Variable & EFPD & $\begin{array}{c}\text { Intercept } \\
\left(\mathrm{a}_{0}\right)\end{array}$ & $\begin{array}{c}\text { Fuel Heat } \\
\text { Rate } \\
\left(a_{1}\right) \\
\end{array}$ & $\begin{array}{c}\text { Gas Gap } \\
\left(\mathrm{a}_{2}\right)\end{array}$ & $\begin{array}{c}\text { Neon } \\
\text { Fraction } \\
\left(a_{3}\right)\end{array}$ & $\begin{array}{c}\text { Graphite } \\
\text { Conduct } \\
\left(a_{4}\right)\end{array}$ & $\begin{array}{c}\text { Fuel } \\
\text { Conduct } \\
\left(\mathrm{a}_{5}\right)\end{array}$ \\
\hline \multicolumn{8}{|l|}{ Capsule 4} \\
\hline VA FT & 58 & -0.184 & 0.455 & 0.249 & 0.601 & -0.075 & -0.044 \\
\hline Peak FT & 58 & -0.025 & 0.484 & 0.204 & 0.525 & -0.095 & -0.092 \\
\hline $\mathrm{TC} 1$ & 58 & -0.313 & 0.361 & 0.356 & 0.627 & -0.027 & -0.003 \\
\hline TC2 (center) & 58 & -0.073 & 0.446 & 0.259 & 0.551 & -0.127 & -0.056 \\
\hline TC3 & 58 & -0.322 & 0.360 & 0.359 & 0.632 & -0.026 & -0.003 \\
\hline VA FT & 303 & 0.197 & 0.599 & 0.210 & 0.208 & -0.116 & -0.096 \\
\hline Peak FT & 303 & 0.345 & 0.592 & 0.174 & 0.171 & -0.129 & -0.151 \\
\hline $\mathrm{TC} 1$ & 303 & -0.135 & 0.567 & 0.404 & 0.251 & -0.068 & -0.017 \\
\hline TC2 (center) & 303 & 0.372 & 0.586 & 0.189 & 0.156 & -0.189 & -0.114 \\
\hline TC3 & 303 & -0.139 & 0.566 & 0.407 & 0.253 & -0.068 & -0.017 \\
\hline VA FT & 601 & -0.228 & 0.552 & 0.159 & 0.661 & -0.093 & -0.050 \\
\hline Peak FT & 601 & -0.063 & 0.539 & 0.138 & 0.574 & -0.099 & -0.088 \\
\hline $\mathrm{TC} 1$ & 601 & -0.523 & 0.512 & 0.385 & 0.704 & -0.066 & -0.011 \\
\hline TC2 (center) & 601 & 0.139 & 0.517 & 0.171 & 0.437 & -0.214 & -0.050 \\
\hline TC3 & 601 & -0.529 & 0.513 & 0.386 & 0.707 & -0.066 & -0.010 \\
\hline \multicolumn{8}{|l|}{ Capsule 6} \\
\hline VA FT & 245 & 0.2747 & 0.4969 & 0.1995 & 0.1415 & -0.0583 & -0.0544 \\
\hline Peak FT & 245 & 0.3746 & 0.5160 & 0.1670 & 0.1224 & -0.0769 & -0.1031 \\
\hline TC1 (center) & 245 & 0.3326 & 0.5001 & 0.1999 & 0.1294 & -0.1010 & -0.0610 \\
\hline TC2 & 245 & 0.1679 & 0.4459 & 0.2613 & 0.1528 & -0.0219 & -0.0061 \\
\hline TC3 & 245 & 0.1480 & 0.4385 & 0.2708 & 0.1564 & -0.0135 & -0.00048 \\
\hline
\end{tabular}




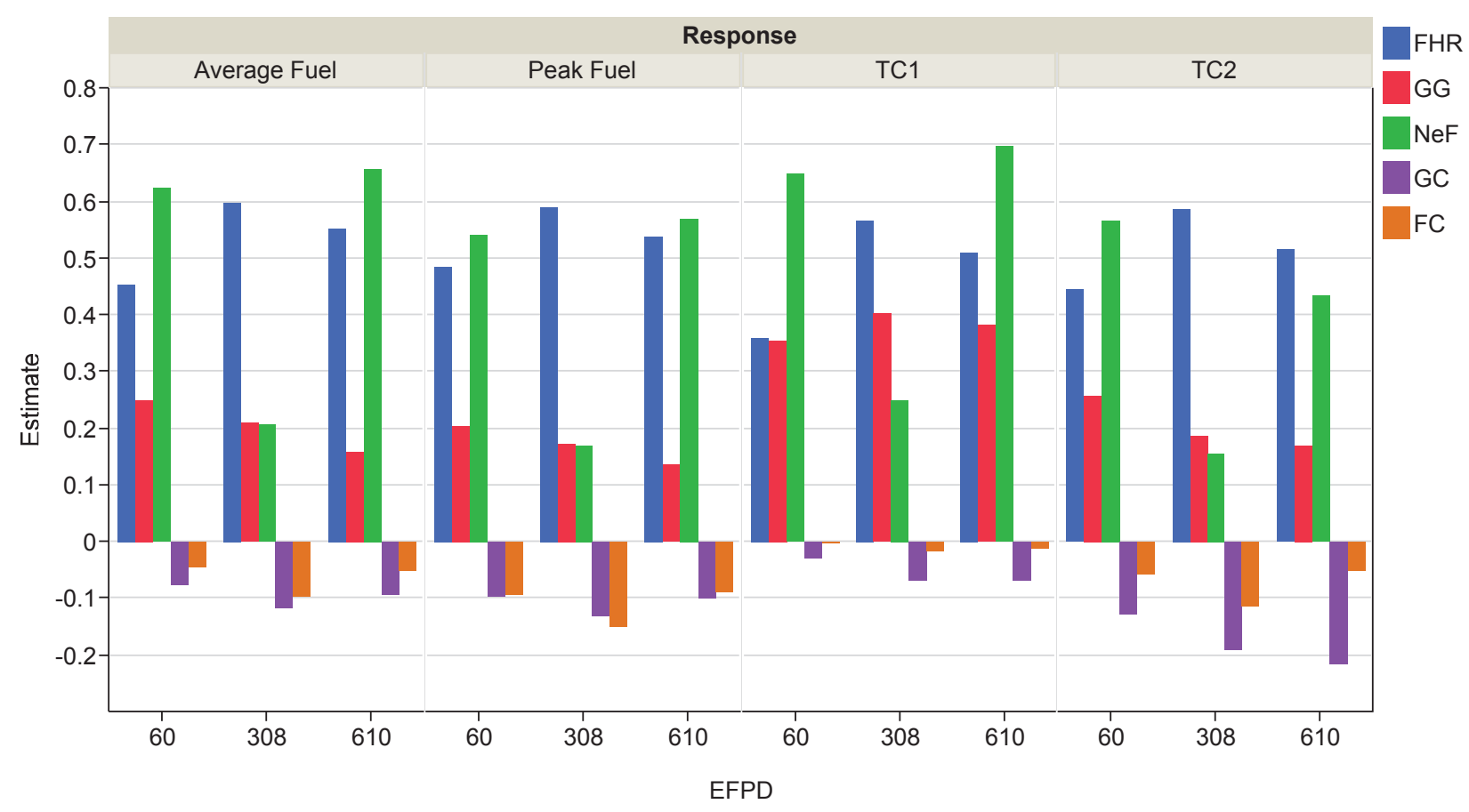

Figure 21. Parameter estimate plots for fuel and TC temperatures in Capsule 4.

\subsubsection{Interpolation of Sensitivity Coefficients for Entire AGR-1 Irradiation}

In this section, the daily sensitivity coefficients for the entire AGR-1 irradiation are estimated from three sensitivity data points in Table 5 by interpolation. Table 6 presents the fuel heat rate values together with the fuel heat rate sensitivity coefficients for peripheral TC1, center TC2, VA FT, and peak FT for the three scenarios for Capsule 4 and the scenario for Capsule 6. The input sensitivity coefficient for TC 1 in Capsule 4 and TC2 in Capsule 6 will apply to all peripheral TCs in other capsules and the sensitivity for TC2 in Capsule 4 and TC1 in Capsule 6 will apply to the center TCs.

Table 6. Sensitivity coefficients of fuel heat rate for TCs, VA FT and peak FT.

\begin{tabular}{|rccccc|}
\hline & \multirow{2}{*}{$\begin{array}{c}\text { Heat Rate } \\
\text { Scenario }\end{array}$} & \multicolumn{4}{c|}{ Sensitivity Coefficients } \\
\cline { 3 - 6 } 1 ( $\left./ \mathrm{cm}^{3}\right)$ & $\mathrm{TC} 1$ & $\mathrm{TC} 2$ (center) & VA FT & Peak FT \\
2 (Capsule 4) & 57.15 & 0.361 & 0.446 & 0.455 & 0.484 \\
3 (Capsule 4) & 111.56 & 0.567 & 0.586 & 0.599 & 0.592 \\
4 (Capsule 6) & 72.39 & 0.512 & 0.517 & 0.552 & 0.539 \\
\hline
\end{tabular}

The fitting procedure of the EXCEL (Excel 2007 SP3) trend line feature is used to construct the polynomial function of input parameter (x) for each input sensitivity (y) based on data in Table 6: (1) fuel heat rate sensitivity as a function of fuel heat rate (Figure 22); (2) neon fraction sensitivity as a function of neon fraction (Figure 23); (3) control gas gap sensitivity as a function of the gap distance (Figure 24); (4) graphite conductivity sensitivity as a function of fast fluence (Figure 25); and (5) fuel compact conductivity sensitivity as a function of fast fluence (Figure 26). The functional form was selected such that: 
- When the extrapolation of the fitted function beyond the range of the three data points is anticipated, the restricted (e.g., no intercept) polynomial and power functions are used to avoid over-fit that may lead to unrealistic extrapolated sensitivity as follows: (a) for fuel heat rate sensitivity, the no-intercept polynomial (Figure 22) is used because it provides the best fit to the four data points and prevented prediction of negative sensitivity at extreme low heat rates during the power-up and power-down of ATR, and (b) for neon fraction, the no-intercept power function (Figure 23) provided the best fit to the three data points, preventing prediction of negative sensitivity coefficients for near zero neon fractions.

- When there is only interpolation needed for estimation of the sensitivity coefficient, the second order polynomial is justified because:

(a) for gas gap distance, the existence of data points for control gas gap sensitivity in Capsule 6, which has the biggest gap in the AGR-1 test train, allows interpolation of the gas gap sensitivity for other time steps and capsules, so the second order polynomial function (Figure 24) can be used to fit the gas gap sensitivity as a function of the gap distance, and

(b) for fuel compact and graphite holder thermal conductivities as functions of fluence, because the level of fluence in the center of Capsule 4 is highest in the AGR-1 test train, the graphite and fuel compact conductivities sensitivity estimations for other time steps and other capsules can be interpolated by second order polynomials.

- The conductivity functions for the graphite holders in Capsules 4 and 6 are in different forms because of different boron carbide concentrations (5.5\% for Capsule 6 and $7 \%$ for Capsule 4 (Demkowicz, Cole, Ploger, \& Winston, 2011)) leading to different sensitivities for these capsules. So the graphite conductivity sensitivity in Capsule 4 will be applied to Capsules 2, 3, 4, and 5 with 7\% boron carbide, and the graphite conductivity sensitivity in Capsule 6 will be applied to Capsule 6 (top) and Capsule 1 (bottom) with 5.5\% boron carbide.

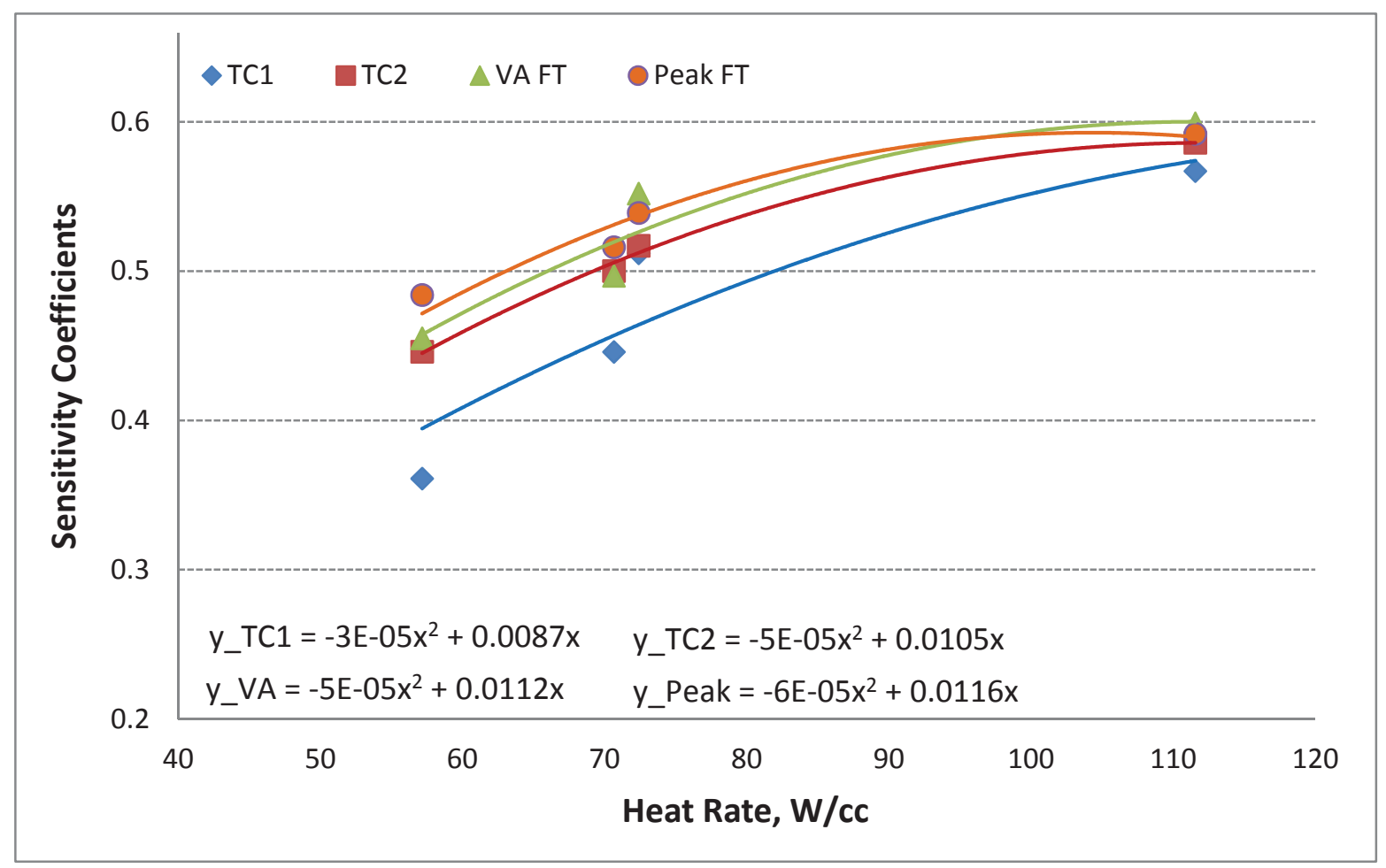

Figure 22. Heat rate sensitivity for TCs and fuel temperatures as function of heat rate. 


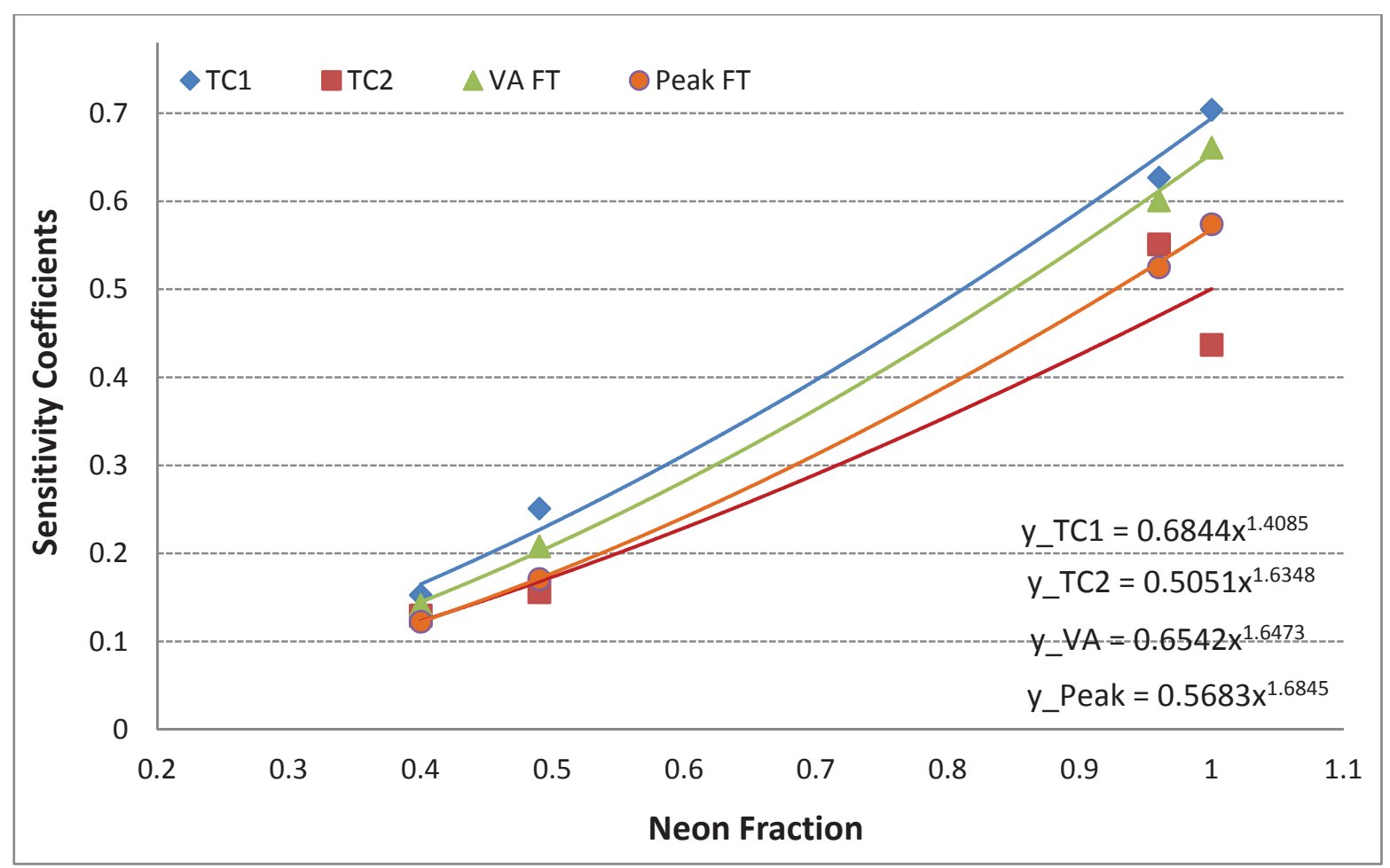

Figure 23. Neon fraction sensitivity for TCs and fuel temperatures as function of neon fraction.

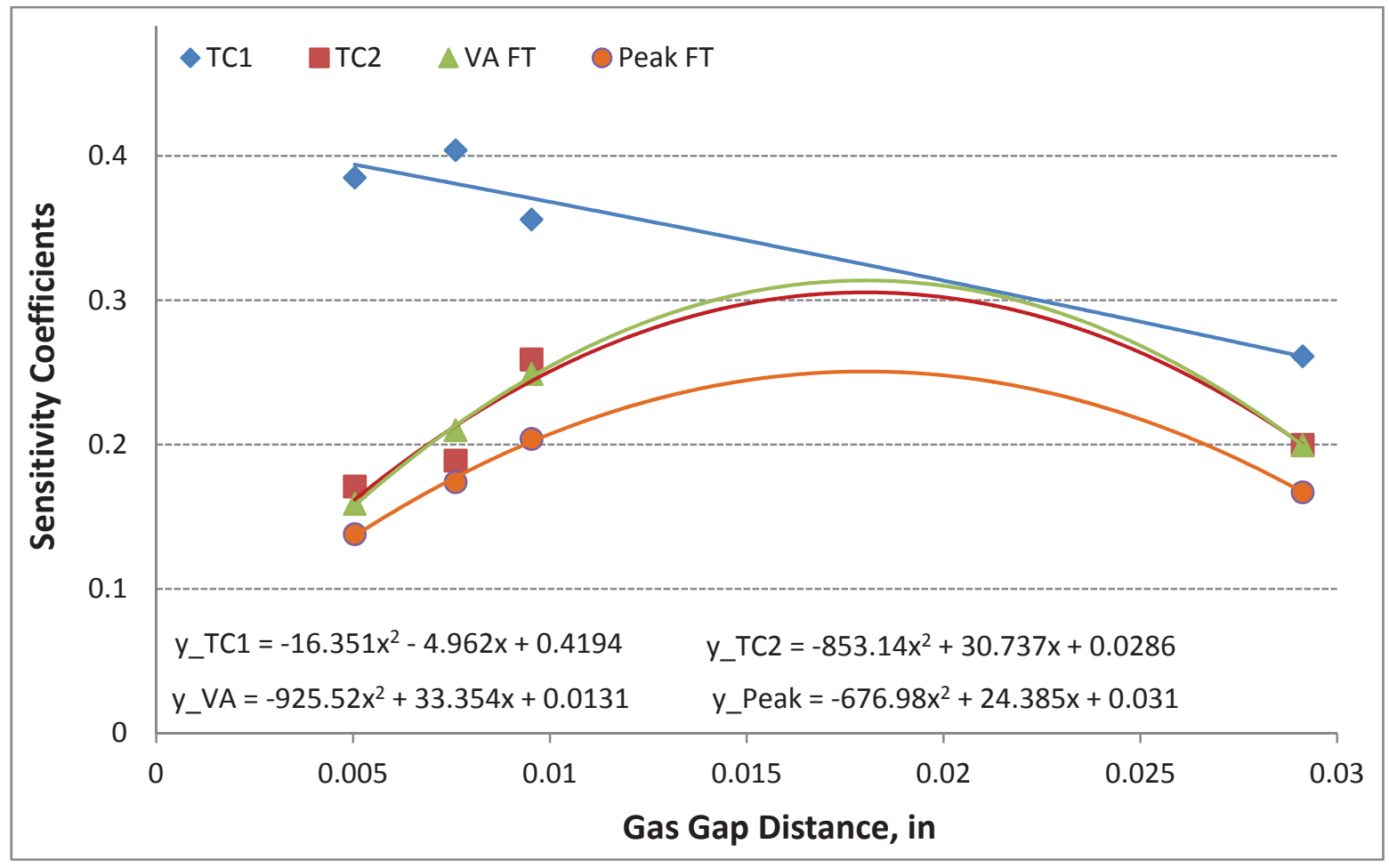

Figure 24. Gas gap sensitivity for TCs and fuel temperatures as function of gap distance. 


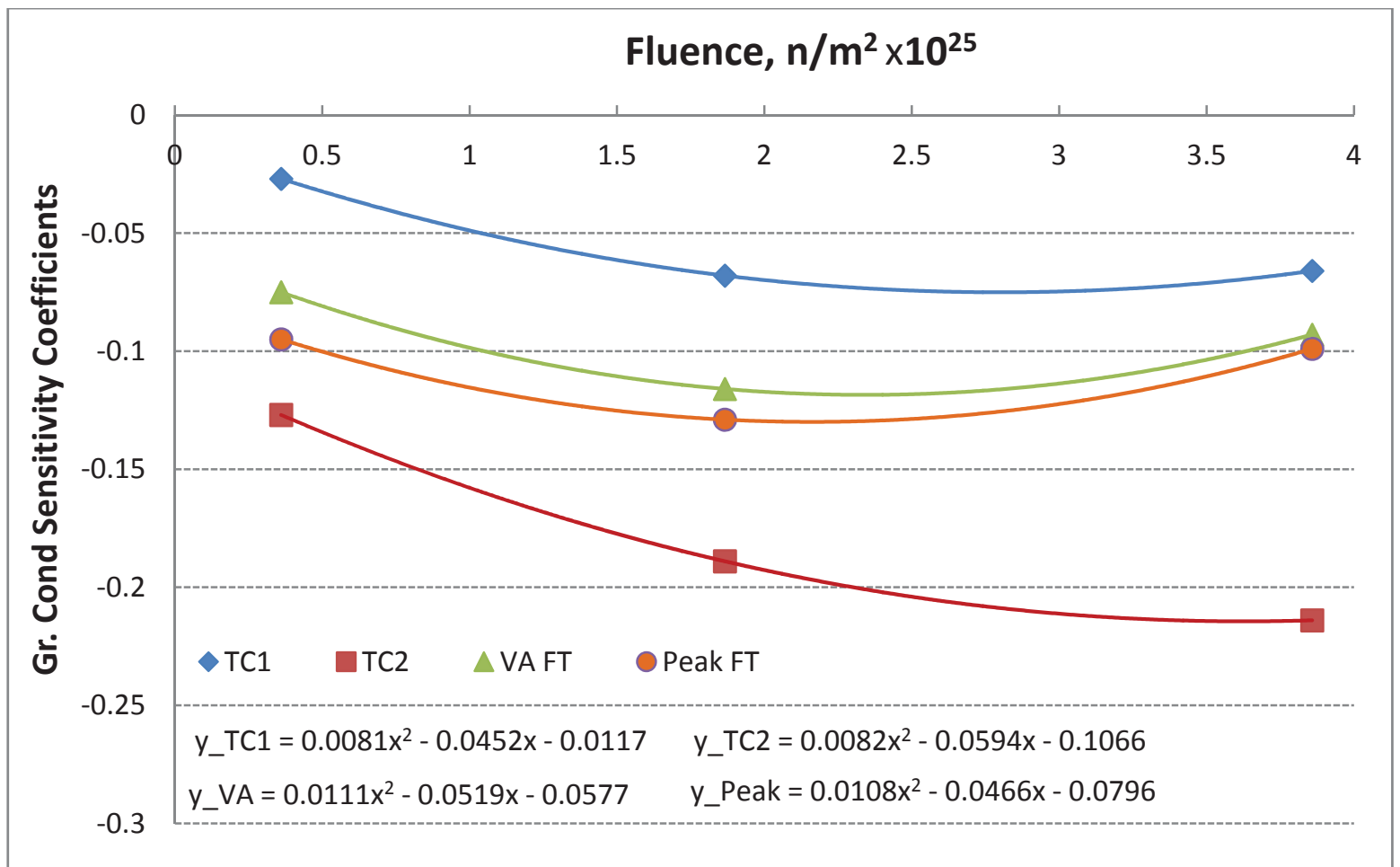

Figure 25. Graphite conductivity sensitivity for TCs and fuel temperatures as function of fluence.

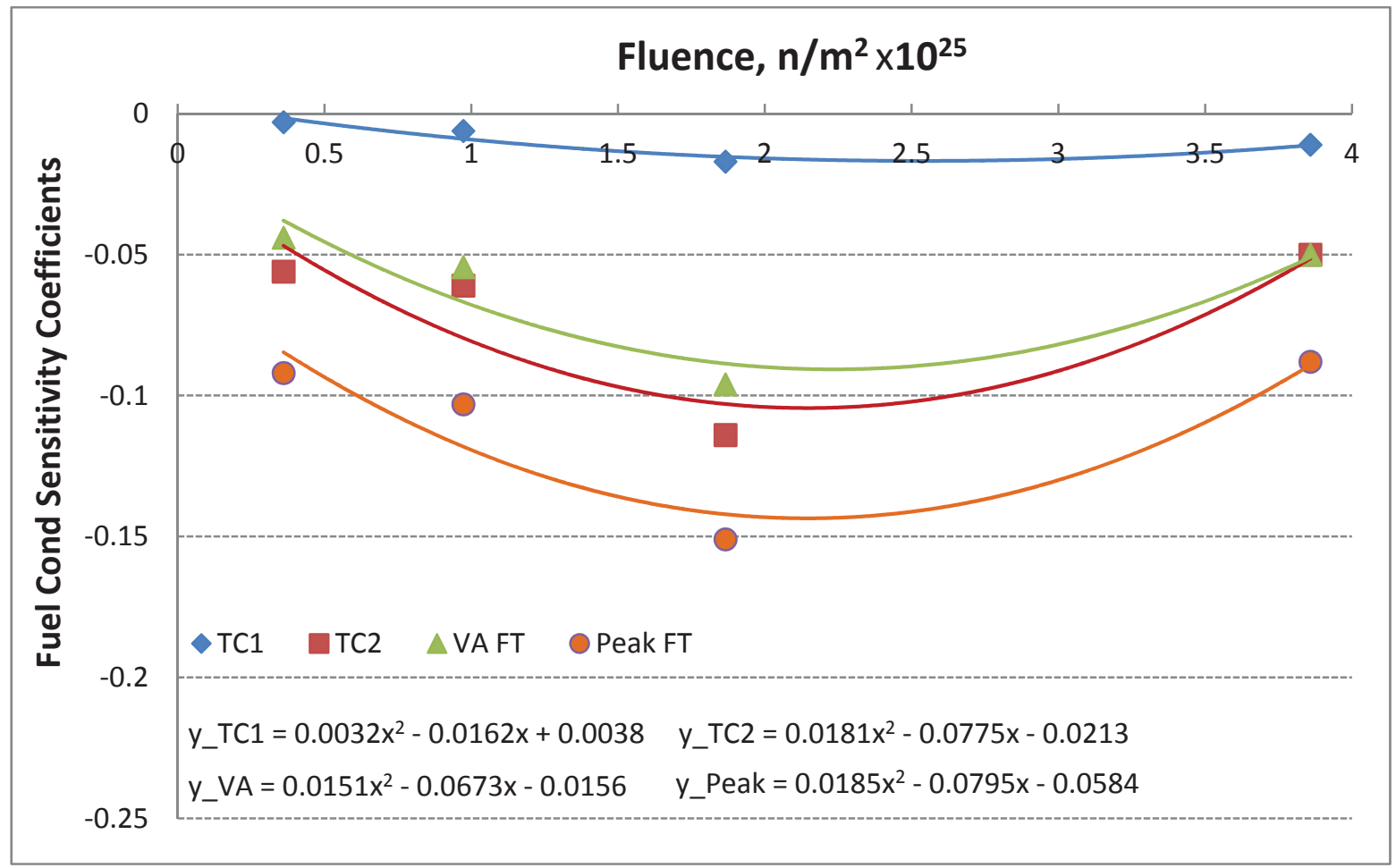

Figure 26. Fuel conductivity sensitivity for TCs and fuel temperatures as function of fluence. 


\subsection{Correlation Coefficients of Thermal Model Input Parameters}

\subsubsection{Uncorrelated Parameter Errors}

The correlation coefficient between two input parameters equals zero when their error sources are independent or uncorrelated. In other words, the variation of one parameter due to its random error does not affect the error of other parameter. For example, initial gas gap distance and neon fraction are both the result of measurements performed by different tools and procedures. The flow rate is measured by the flow meter and the control gas gap is measured by the ruler. Therefore, the error of the gas gap measurement does not affect the error in flow rate measurement and these errors are considered independent. As a result, the correlation coefficient of neon fraction and gas gap distance is zero. For the same reason, correlation coefficients between the two measured parameters, gas gap distance and neon fraction, and three calculated parameters, fuel heat rate, graphite, and fuel compact thermal conductivities, are zero.

\subsubsection{Correlated Parameter Errors}

This section estimates the correlation coefficients between error sources of three calculated parameters: fuel heat rate, graphite conductivity, and fuel compact conductivity. The error associations of these calculated parameters are caused by the fact that their calculation formulas contain common factors such as temperature and fast neutron fluence.

\subsubsection{Graphite and Fuel Compact Thermal Conductivities}

The graphite thermal conductivity and fuel compact thermal conductivity plots varying with temperature and fast fluence (expressed in $\mathrm{n} / \mathrm{cm}^{2}$ or equivalent $d p a$ ) are presented in Figure 16 and Figure 17, respectively. From available data provided by the look-up tables for fuel and graphite conductivities as functions of fast fluence and temperature (Hawkes G. , 2011b), the relationship of graphite and fuel compact thermal conductivities as functions of fast fluence (converted into $\mathrm{dpa}$ ) and temperature $(T)$ are constructed using the JMP software. The functional relationship given for graphite thermal conductivity is:

$$
\begin{aligned}
G C= & 3.36 \cdot 10^{-4}-3.85 \cdot 10^{-8} T+1.02 \cdot 10^{-8}(T-1449.5)(\log (d p a)+5.05) \\
& -4.499 \cdot 10^{-5} \log (d p a)-2.36 \cdot 10^{-6}(\log (d p a)+5.05)^{2}
\end{aligned}
$$

and for fuel compact conductivity is:

$$
\begin{aligned}
F C= & 3.72 \cdot 10^{-5}+4.07 \cdot 10^{-8} T-9.44 \cdot 10^{-6} d p a+3.74 \cdot 10^{-11}(T-2030)^{2} \\
& +2.31 \cdot 10^{-8}(T-2030)(d p a-1.82)+2.85 \cdot 10^{-6}(d p a-1.82)^{2}
\end{aligned}
$$

Using the JMP software, a total of 100,000 data points was collected from normal distributions of AGR-1 fuel temperature $[1500 \pm 200 \mathrm{~K}]$, graphite temperature $[1200 \pm 200 \mathrm{~K}]$, and $d p a\left[\mu_{\mathrm{dpa}} \pm 10 \%\right]$ for five levels of the mean $d p a, \mu_{\mathrm{dpa}}=0.5,1,2,3,4$ corresponding to fast neuron fluence. The multiplier used to convert fast fluence to $d p a$ is $0.823 \times 10^{25} \mathrm{dpa} /\left(\mathrm{n} / \mathrm{m}^{2}\right)$ (Hawkes G.L., 2011).

In order to compute the noise correlation coefficient between fuel and graphite conductivities, the fuel and graphite temperatures of one data point are sampled with the same noise components. The sampled $d p a$ and temperature values are inserted in (Eq. 11) and (Eq. 12) to compute the graphite thermal conductivity and the fuel compact thermal conductivity for each data point. The correlation coefficient between graphite and fuel compact thermal conductivities is estimated using the JMP "multivariate" function. Figure 27 shows the scatter plot matrix of fuel temperature, $d p a$, fuel and graphite thermal 
conductivities together with their distribution and correlation coefficients of each pair of variables for $d p a$ levels 0.5 and 2.0 .
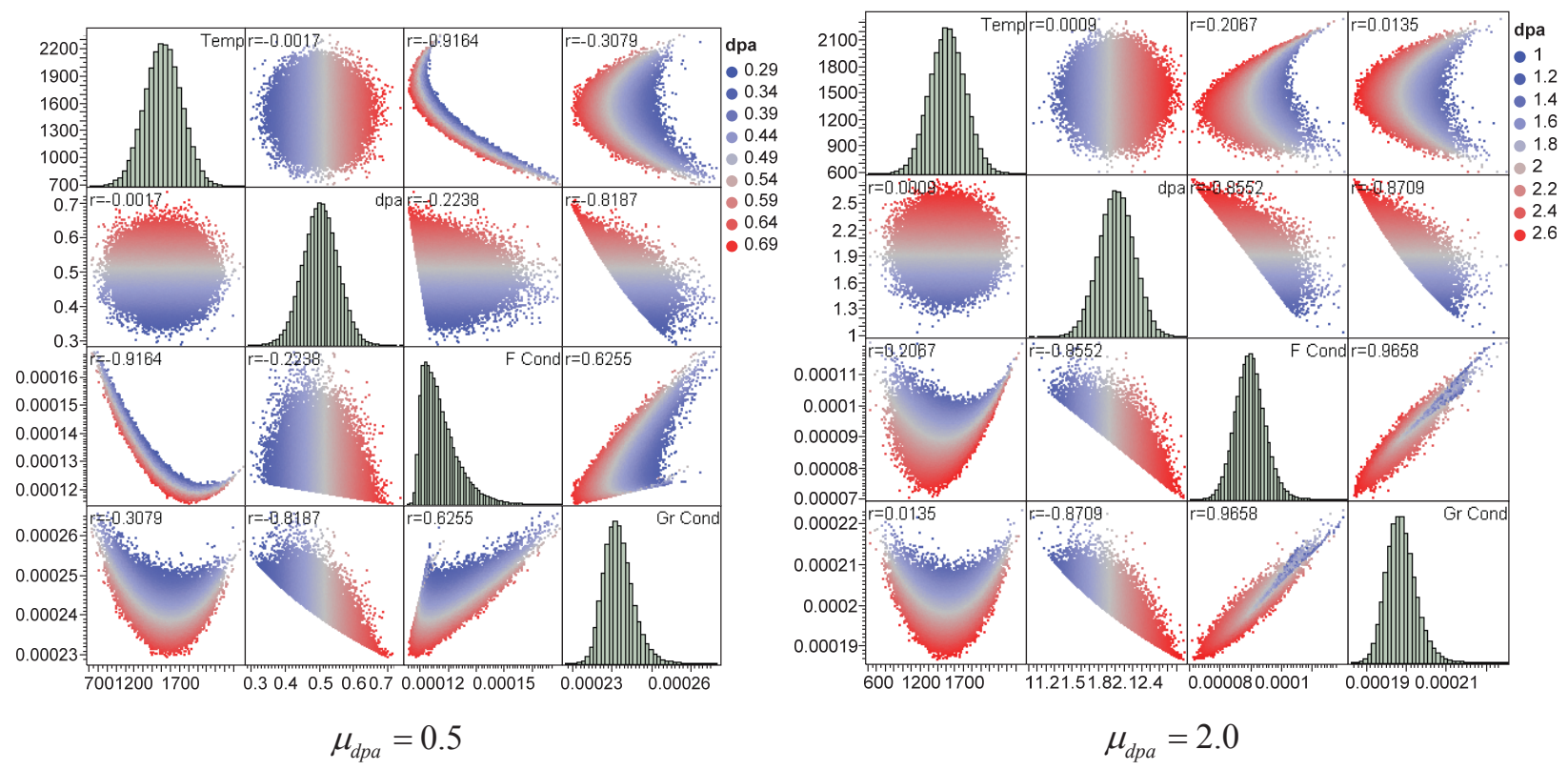

Figure 27. Scatter plot matrix of fuel temperature, dpa, fuel and graphite thermal conductivities.

The noise correlation coefficients between fuel and graphite thermal conductivities $\rho_{G C, F C}$ for five levels of fast neutron fluence (converted from $d p a$ ) are presented in Table 7 and Figure 28. The second order polynomial function of the fast fluence $(f)$ in $\mathrm{n} / \mathrm{m}^{2} \times 10^{25}$ was used to fit the correlation coefficients in Table 7 as:

$$
\rho_{G C, F C}=-0.0708 f^{2}+0.3585 f+0.4464
$$

Table 7. Correlation coefficients between graphite and fuel thermal conductivities.

\begin{tabular}{|l|c|c|c|c|c|}
\hline fluence $\left(\mathrm{n} / \mathrm{m}^{2} \times 10^{25}\right)$ & 0.6075 & 1.215 & 2.430 & 3.645 & 4.860 \\
\hline Correlation coefficient & 0.6255 & 0.7739 & 0.9658 & 0.7355 & 0.544 \\
\hline
\end{tabular}




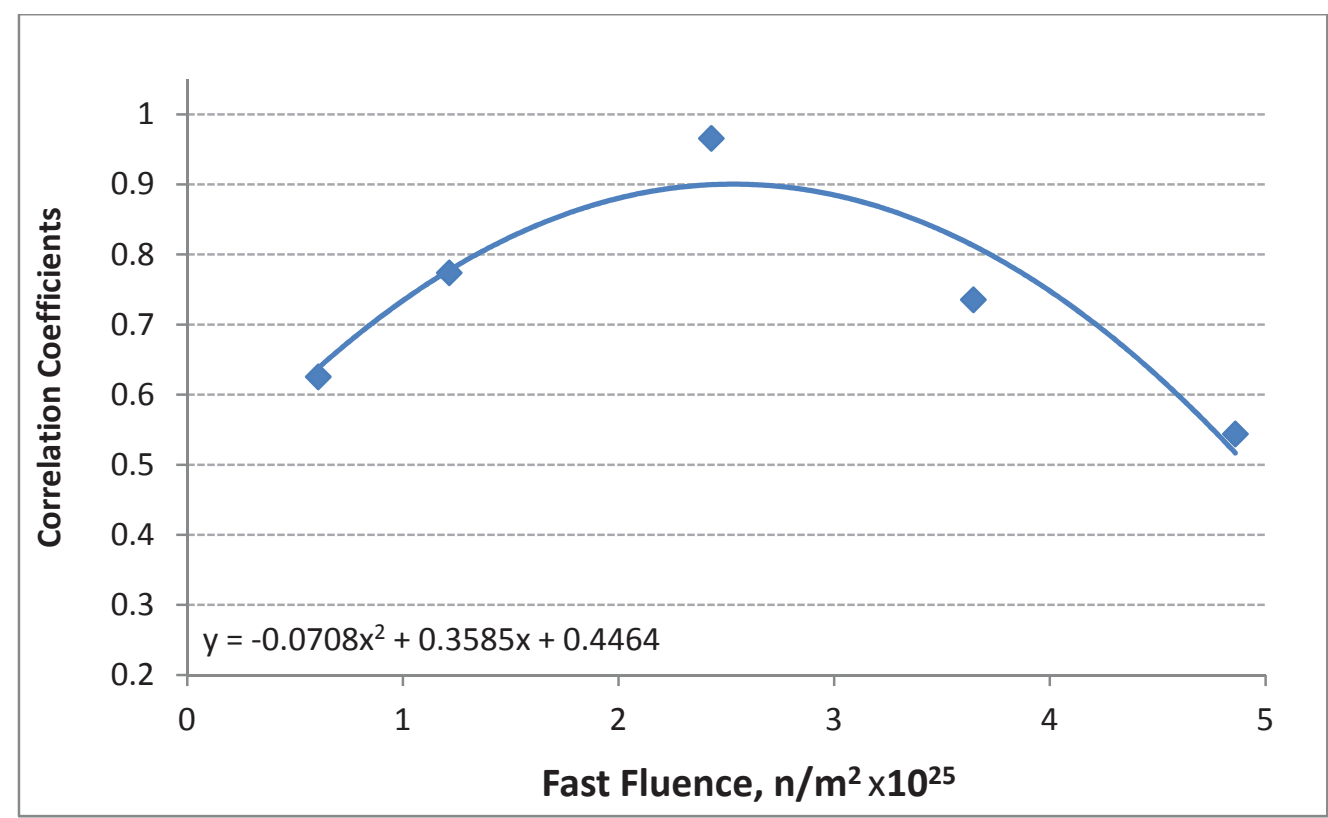

Figure 28. Correlation coefficients between graphite and fuel thermal conductivities varying with fast neutron fluence.

\subsubsection{Graphite/Fuel Compact Thermal Conductivity and Fuel Compact Heat Rate}

The value of fuel compact heat rate $(Q)$ depends on the daily neutron flux, fuel temperature, and material properties such as cross-section and fuel burnup. The graphite and fuel compact thermal conductivity, as mentioned earlier, is a function of temperature and neutron fast fluence. Based on the functional dependence of fuel compact and graphite thermal conductivity and fuel heat rate on neutron fast fluence and neutron flux respectively, it will be reasonable to assume that the correlation coefficient between their noises should be smaller than between graphite and fuel conductivities. Since the fuel compact and graphite thermal conductivities and fuel heat rate are not possible to simulate, their noise correlation coefficients will assume to be half of the correlation coefficient between fuel and graphite conductivities. 


\section{PROPAGATION OF PARAMETER UNCERTAINTIES AND SENSITIVITIES}

\subsection{Propagation Formula}

The variation of parameter estimates across three thermal condition scenarios in Capsule 4 presented in Figure 21 reflects the variation in parameter sensitivities over the entire input domain for AGR-1. This confirms the nonlinear nature of Capsule 4 thermal models as a function of the five input variables. However, within a small enough input range, e.g., from [nominal*(1-10\%)] to [nominal* $(1+10 \%)]$, the AGR-1 predicted temperature can be estimated by a linear combination of five input variables as presented in Eq. 10. Subsequently, the standard parameter error propagation of the linear summation (Ostle \& Mensing, 1975) can be used to calculate the overall output temperature uncertainty from the variance-covariance matrix of input variables for an input range close to the nominal values. The expression of output variance for each output, $T$, is given in Section 2.1 and reproduced here:

$$
\sigma_{T}^{2}=\sum_{i}^{n} a_{i}^{2} \sigma_{i}^{2}+\sum_{i}^{n} \sum_{j \neq i}^{n} \rho_{i j} a_{i}^{2} \sigma_{i}^{2} a_{j}^{2} \sigma_{j}^{2}
$$

where: $\sigma_{T}$ is the overall uncertainty in terms of standard deviation,

$a_{i}$ is the sensitivity coefficient for parameter $i$,

$\sigma_{i}$ is the uncertainty of input parameter $i$ in terms of standard deviation,

$\rho_{i j}$ is the correlation coefficient for input parameters $i$ and $j$.

The input parameter uncertainties are estimated in terms of relative standard deviations (\%) in Section 2.4 and the sensitivity coefficients are estimated in Section 2.5. Section 2.6 addresses the correlation coefficients of each variable pair. The correlation coefficient of a variable pair is a measure of the degree (or intensity) of association between two variables (Ostle \& Mensing, 1975).

\subsection{Uncertainty Propagation for Daily Average Temperatures}

For each capsule, the AGR-1 thermal model parameter uncertainty quantification for daily average temperatures (TCs, VA FT, or peak FT) is performed using the results presented in Section 2.

\subsubsection{Daily Input Parameter Uncertainties $\left(\sigma_{i}\right)$}

The daily input parameter uncertainties in terms of relative standard deviation for the five significant input variables are estimated as discussed in Section 2.4. The details of the daily uncertainty calculations are:

- The parameter uncertainties for fuel heat rate, graphite holder thermal conductivity and fuel compact thermal conductivity are assumed to be constant for all time steps and six capsules as presented in Table 1. The parameter uncertainties given are the random errors, except for an additional positive $10 \%$ bias in fuel heat rate for Capsule 6 . Therefore, their plots as a function of EFPDs are flat lines for all three parameters in all capsules, except fuel heat rate in Capsule 6.

- The daily parameter uncertainties for gas gap distance $\left(\sigma_{G G i}\right)$ are based on the error propagation of the gap distance formula of Eq. 5 as follows: 
$\sigma_{G G_{i}}=\sqrt{\left[\sigma_{G G_{S}}\left(1-\frac{\text { fluence }_{i}}{\text { fluence }_{A G R}}\right)\right]^{2}+\left[\sigma_{G G_{e}} \frac{\text { fluence }_{i}}{\text { fluence }_{A G R}}\right]^{2}}$

where $\sigma_{G G s}, \sigma_{G G e}$ are the gap distance uncertainty at the start and the end of the AGR-1 irradiation, respectively. The calculated daily gap uncertainties using Eq. 15 are based on following assumptions:

a. The uncertainties of the final gap distance are assumed to be $50 \%$ more than the uncertainties of the initial gap distance presented in Table 2 .

b. The daily gap uncertainty at time step $(i)$ is greater than or equal to the initial gap uncertainty.

Because of assumption (b), when $\sigma_{G G i}$, calculated by Eq. 15, is less than the initial gap uncertainty, it is set to $\sigma_{G G s}$, i.e., $\sigma_{G G i}=\sigma_{G G s}$. Therefore, the gap uncertainty plot is flat during the first half of the AGR-1.

- The daily parameter uncertainty for neon fraction $\left(\sigma_{\text {Nef }_{i}}\right)$ is calculated for each time step and each capsule by substituting the actual capsule neon fraction on that time step using Eq. 7 (representing a functional relationship between neon fraction uncertainties and neon fraction values). Since the capsule neon fractions vary with time in order to maintain the capsule specified fuel temperature, the daily uncertainties of neon fraction also vary with EFPDs.

\subsubsection{Daily Parameter Sensitivities $\left(a_{i}\right)$}

The daily parameter sensitivities, $a_{i}$, of five significant input variables at time step (i) are estimated using the functional relationships between sensitivity coefficients and corresponding input parameters established in Section 2.5 for each output temperature in each capsule. Since the inputs to the thermal models vary with time, the resulting daily parameter sensitivities also vary with time.

\subsubsection{Daily Correlation Coefficients $\left(\sigma_{F C, G C_{i}}\right)$}

The daily correlation coefficients between fuel compact and graphite thermal conductivities, $\rho_{F C, G C}$, at time step (i), are calculated using the function of fast neutron fluence given in Eq. 13 and shown in Figure 28. Since the neutron fluence varies with time, the daily correlation coefficients also vary with time.

\subsubsection{Overall Parameter Uncertainty $\left(\sigma_{T i}\right)$}

The overall uncertainty (in terms of standard deviation) of the thermal model output temperature at time step (i) is calculated using Eq. 14, which is based on propagation of Eq. 10:

$$
\sigma_{T_{i}}=\sqrt{\left(a_{H R_{i}} \sigma_{H R_{i}}\right)^{2}+\left(a_{G G_{i}} \sigma_{G G_{i}}\right)^{2}+\left(a_{N e f_{i}} \sigma_{N e f_{i}}\right)^{2}+\left(a_{G C_{i}} \sigma_{G C_{i}}\right)^{2}+\left(a_{F C_{i}} \sigma_{F C_{i}}\right)^{2}+2 \rho_{F C, G C_{i}} a_{G C_{i}} a_{F C_{i}} \sigma_{G C_{i}} \sigma_{F C_{i}}}
$$

Since the input sensitivity, correlation coefficient and uncertainty vary with time, the calculated temperature uncertainty also varies with time (EFPD). 


\subsection{Uncertainty Quantification for Time Average (TA) Temperatures}

The capsule TA VA FT and TA peak FT at day $(i)$ are calculated from daily average temperatures as

$$
T_{T A_{i}}=\frac{\sum_{k=1}^{i} T_{k} \cdot t_{k}}{\sum_{k=1}^{i} t_{k}}
$$

where $T_{k}$ and $t_{k}$ are daily average calculated temperature and time step at day $(k)$. Time step $t_{k}$ equals 1 for most time steps and $t_{k}<1$ for the startup or power-down days. The error standard deviation of the TA temperatures is calculated using the standard formula (Eq. 14) for error propagation of the linear combination of daily average temperatures. Assuming that the errors of daily averaged calculated temperatures are independent from each other, the error standard deviation of the TA FT (VA or peak) is calculated as

$$
\sigma_{T_{T_{i}}}=\sqrt{\frac{\sum_{k=1}^{i}\left(t_{k} \sigma_{T_{k}}\right)^{2}}{\sum_{k=1}^{i} t_{k}}}
$$




\section{RESULTS}

The weighted parameter variance $\left(a_{i} \sigma_{i}\right)^{2}$ is the square of the product of the sensitivity coefficient and the standard deviation for a parameter. The dominant parameter of the predicted temperature uncertainty is the parameter with the largest weighted parameter variance. It varies depending on input parameters (capsule thermal conditions) and output temperatures (TC, volume-average, and peak). The results given in the following sections identify the dominant parameter and quantify its influence on the overall uncertainty.

\subsection{Temperature Uncertainty Results for Capsule 6}

The overall model temperature standard deviation is estimated using the uncertainty quantification procedure described in the previous sections. The daily uncertainty (in terms of standard deviations at each time step) is estimated for six calculated temperatures: center TC1, peripheral TC2, daily average VA FT, daily average peak FT, TA VA FT, and TA peak FT.

Table 8 summarizes the results for Capsule 6. The dominant parameter is the input parameter with the largest influence on the overall uncertainty. The dominant sensitivity is the range of the sensitivity coefficients for the driver over all EFPDs. The dominant uncertainty is the range of the relative standard deviations of the dominant. The overall uncertainties are given both as a range over EFPDs and at the end of irradiation.

Table 8. Summary of temperature uncertainty results for Capsule 6

\begin{tabular}{|c|c|c|c|c|c|c|}
\hline & Center TC1 & $\begin{array}{l}\text { Peripheral } \\
\text { TC2 }\end{array}$ & VA FT & Peak FT & $\begin{array}{c}\text { TA VA } \\
\text { FT at end }\end{array}$ & $\begin{array}{l}\text { TA Peak } \\
\text { FT at end }\end{array}$ \\
\hline Dominant parameter & $\begin{array}{l}\text { Fuel heat } \\
\text { rate }\end{array}$ & $\begin{array}{l}\text { Fuel heat } \\
\text { rate }\end{array}$ & $\begin{array}{l}\text { Fuel heat } \\
\text { rate }\end{array}$ & $\begin{array}{l}\text { Fuel heat } \\
\text { rate }\end{array}$ & $\begin{array}{l}\text { Fuel heat } \\
\text { rate }\end{array}$ & $\begin{array}{l}\text { Fuel } \\
\text { heat rate }\end{array}$ \\
\hline Don & $0.30-0.56$ & $0.28-0.5$ & $0.32-0.60$ & $0.33-0.56$ & & \\
\hline Dominant uncertainty, $\%$ & 10.3 & 10.3 & 10.3 & 10.3 & & \\
\hline Overall uncertainty, $\%$ & $3.5-6.1$ & $3.0-5.7$ & $3.5-5.75$ & $3.5-5.5$ & 5.0 & 5.0 \\
\hline Overall uncertainty, ${ }^{\circ} \mathrm{C}$ & $20-78$ & $17-55$ & $20-74$ & $25-79$ & 55 & 60 \\
\hline
\end{tabular}

Since Capsule 6 is located at the top of the AGR-1 test train where the incident neutron flux from the ATR core on its fuel compacts is lowest, this capsule has the largest control gap distance and low boron carbide concentration (5\%) in the graphite holder. Properties specific to Capsule 6 lead to the following for temperature uncertainty quantification of this capsule:

1. The daily input standard deviations for five significant input parameters for Capsule 6 are presented in Figure 29. For a few time steps, usually at the beginning and the end of each ATR cycle, the neon fractions were near zero. Therefore the relative neon fraction uncertainties at these time steps are unusually large and out of the plotting limits as shown by the green line in Figure 29.

Since the fuel fission heat rate uncertainty in Capsule 6 includes a positive $10 \%$ bias, the combined standard deviation of fuel heat rate in Capsule 6 calculated by Eq. 19 will be $10.3 \%$ instead of the random error of $2.5 \%$ in the other five capsules.

$$
\sigma_{H R}=\sqrt{\sigma_{\text {bias }}^{2}+\sigma_{\text {random }}^{2}}=\sqrt{10^{2}+2.5^{2}}=10.3 \%
$$

The standard deviation of control gas gap distance (blue line in Figure 29) is smallest among the six capsules (3.5\% for initial gap distance) because Capsule 6 has the biggest gas gap. 
2. The daily input sensitivities for VA FT, peak FT, and temperatures at TC locations as function of EFPDs are plotted in Figure 30.

Figure 31 plots together the sensitivity of graphite thermal conductivity estimated using the data from ABAQUS runs for Scenario 4 in Capsule 6 (empty symbols) and the graphite conductivity sensitivity calculated using the ABAQUS runs data for Scenarios 1 to 3 for Capsule 4 (solid symbols).

According to this figure, the sensitivities for Capsule 6 temperatures are clearly lower than for Capsule 4 temperatures. This difference of sensitivities in the two capsules was caused by different boron carbide concentrations in Capsule 4 (7\%) and Capsule $6(5.5 \%)$. As a result, the sensitivity of graphite conductivity in Capsule 6 is reduced. The reduction in coefficients is equal to the ratios between the sensitivity of graphite conductivities for Capsule 6 and graphite conductivity sensitivities estimated using functions established from the three scenarios for Capsule 4 at the same level of fast fluence $\left(\sim 1 \times 1025 \mathrm{n} / \mathrm{m}^{2}\right)$. The Capsule 6 coefficients are 0.456 for peripheral TC, 0.645 for center $\mathrm{TC}, 0.597$ for volume averaged fuel temperature, and 0.670 for peak fuel temperature.

3. The daily relative and absolute standard deviations of TC and fuel compact temperature model uncertainty are presented in Figure 32 and Figure 33. The lower uncertainty standard deviations at the beginning and the end of each ATR cycle are because of lower input values leading to lower parameter sensitivities for these time steps.

The dominant factor for model temperature uncertainties in Capsule 6 is the $10 \%$ bias error in the fuel heat rate (highest sensitivity coefficient and high input uncertainty), which contributed up to 6\% (or $75^{\circ} \mathrm{C}$ ) of peak fuel temperature and central $\mathrm{TC} 1$ relative standard deviations.

4. The daily temperature and one standard deviation of the predicted temperatures in Capsule 6 are plotted for central TC1 and peripheral TC2 in Figure 34, daily average VA FT and daily peak FT in Figure 35, and TA VA FT and TA peak FT in Figure 36. The model temperatures at the center capsule location (TC1) have the highest uncertainty of $6.1 \%$.

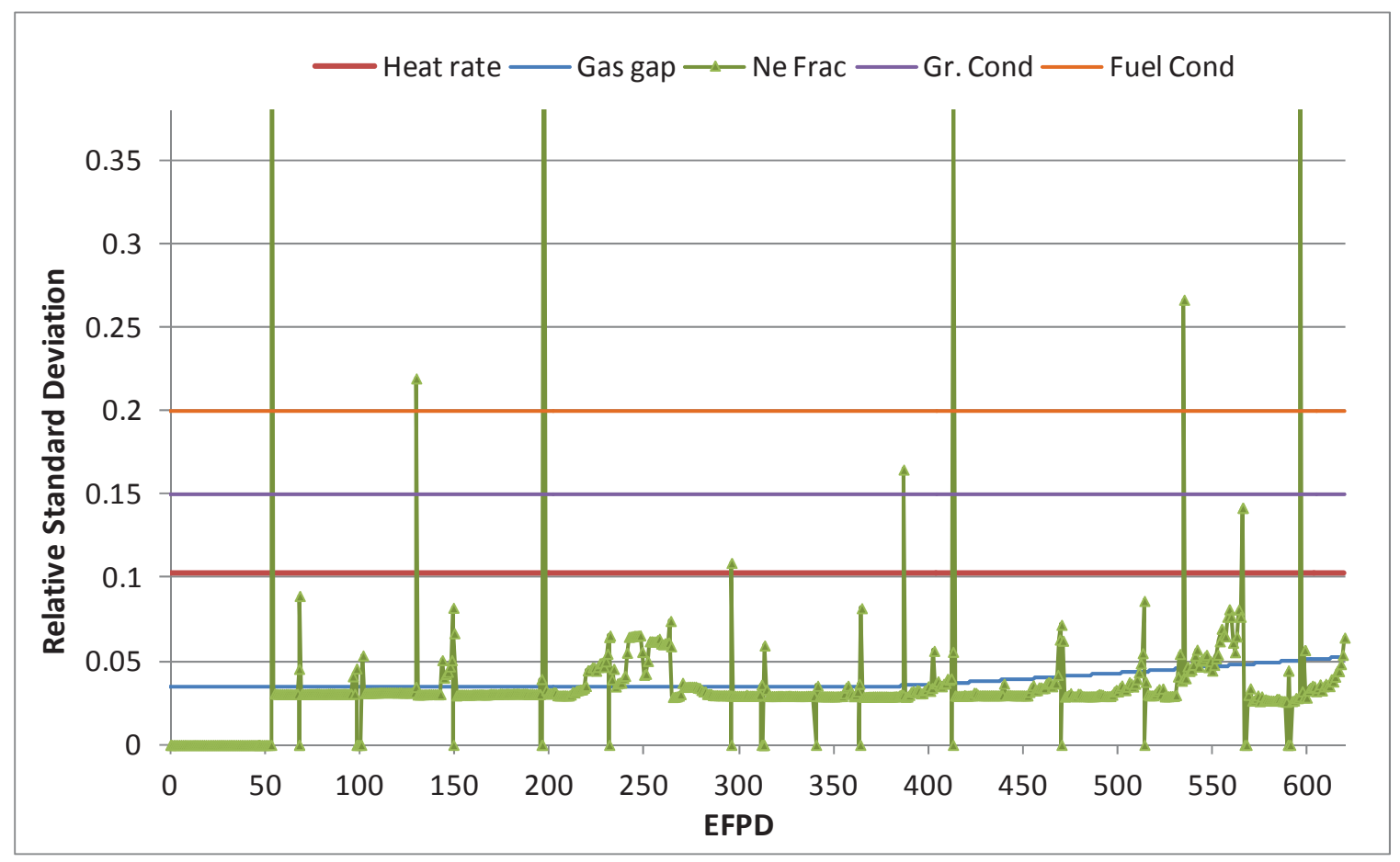

Figure 29. Daily input relative standard deviations for Capsule 6. 

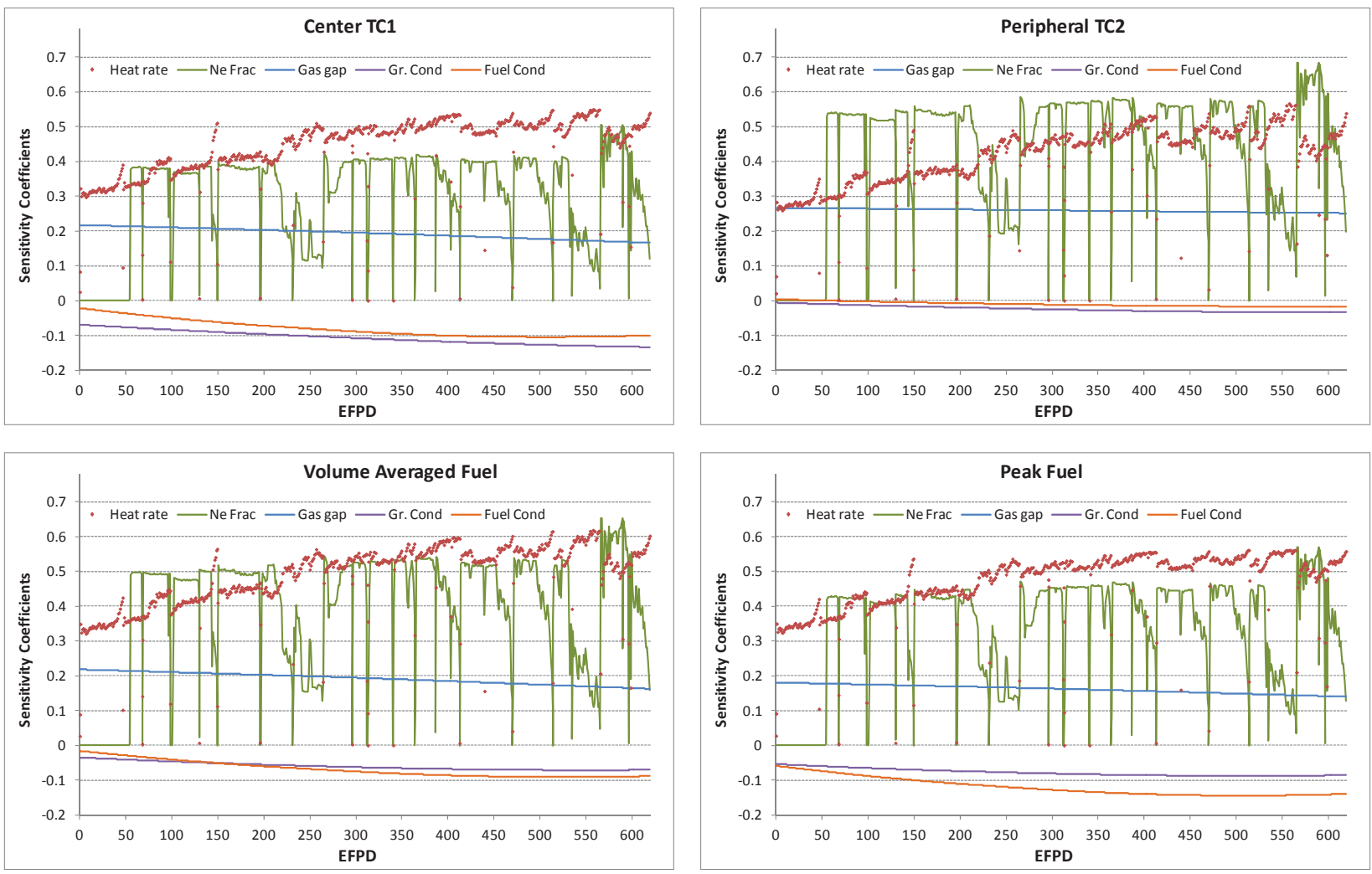

Figure 30. Daily input parameter sensitivities for temperatures in Capsule 6.

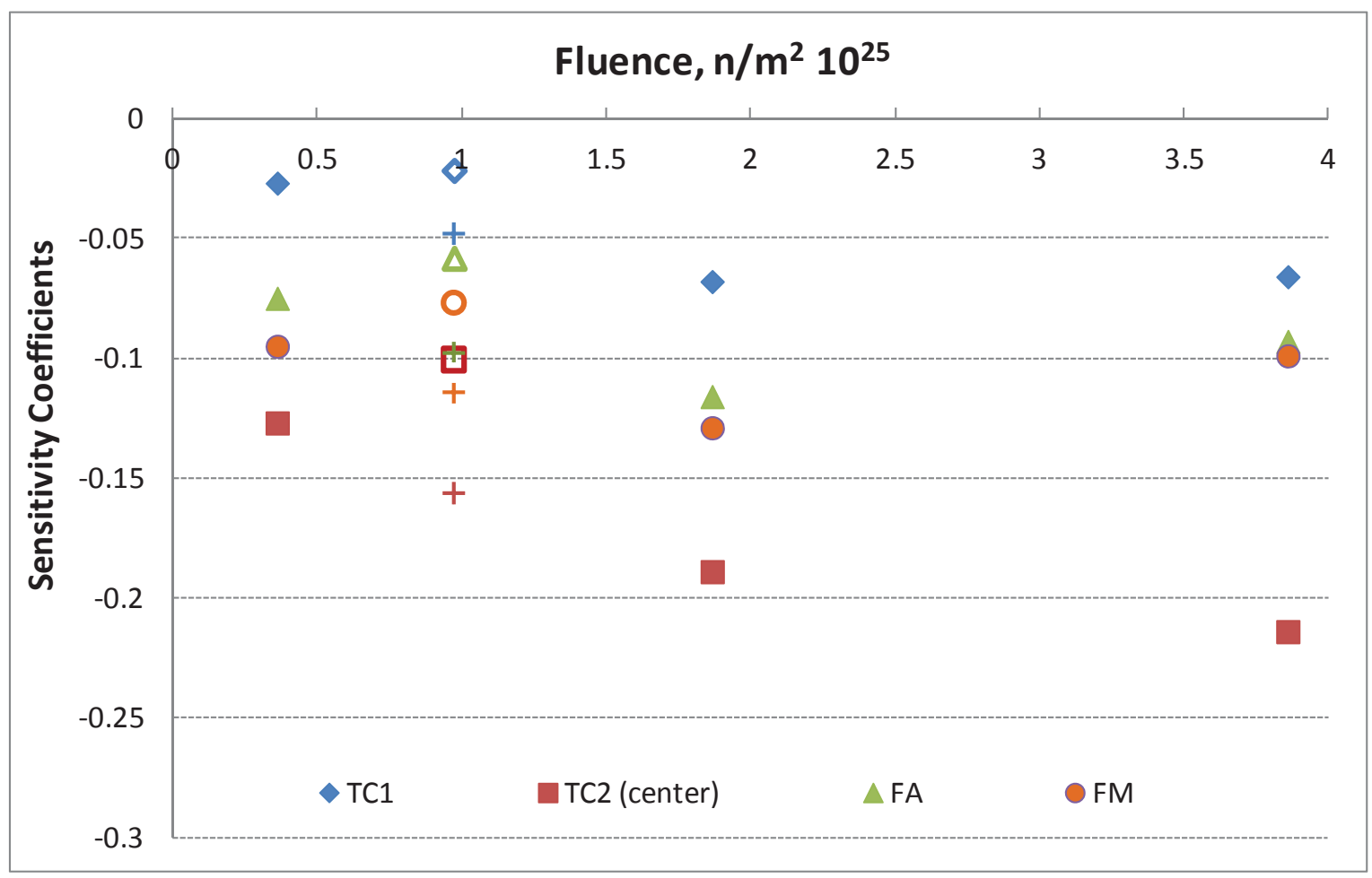

Figure 31. Model sensitivities of graphite conductivity: Capsule 6 actual data (empty symbols) versus predicted (cross symbol) based on Capsule 4 actual data (solid symbols). 


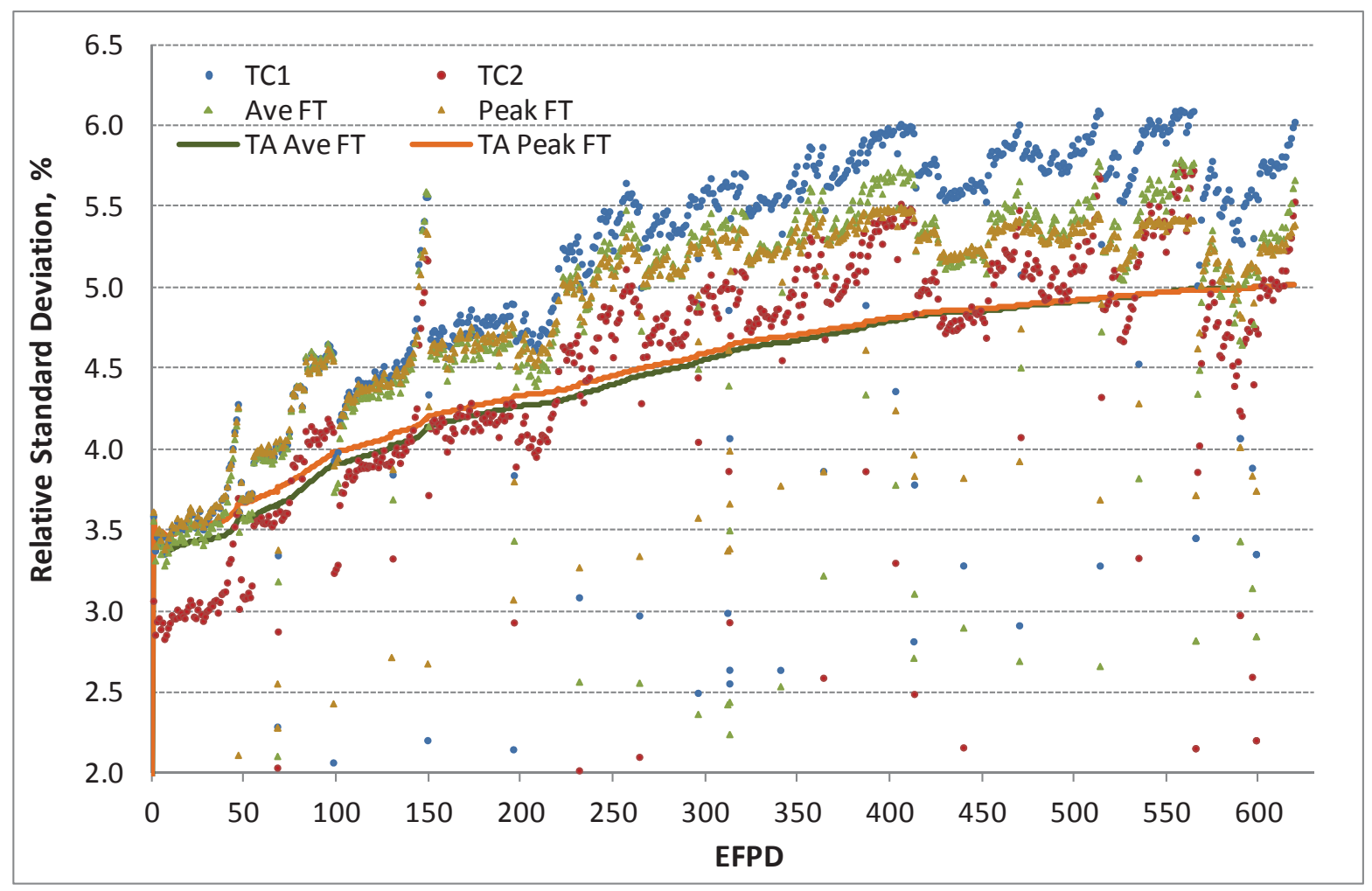

Figure 32. Daily relative standard deviations of predicted TC and fuel temperatures in Capsule 6.

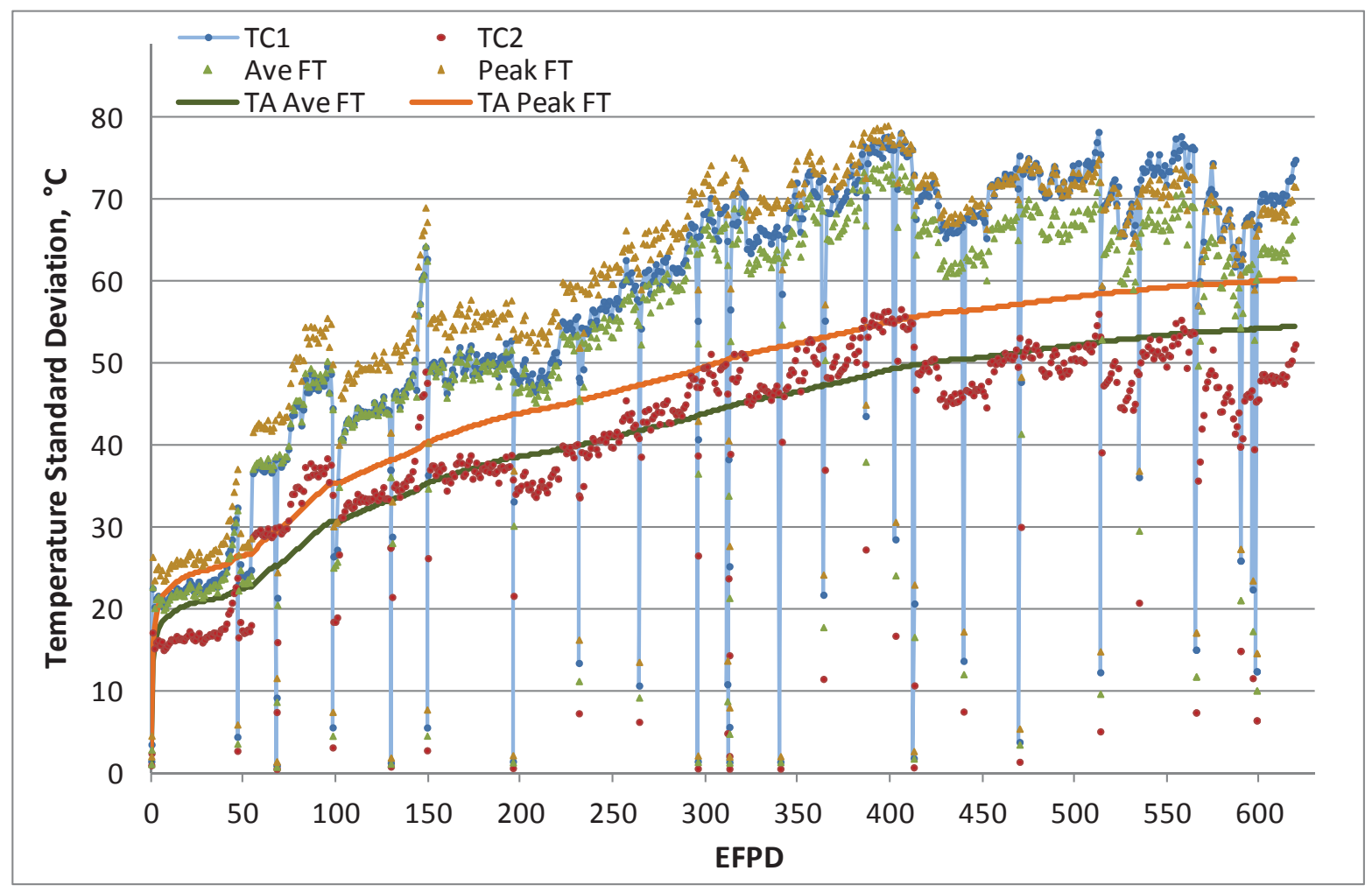

Figure 33. Daily standard deviations of predicted TC and fuel temperatures in Capsule 6. 


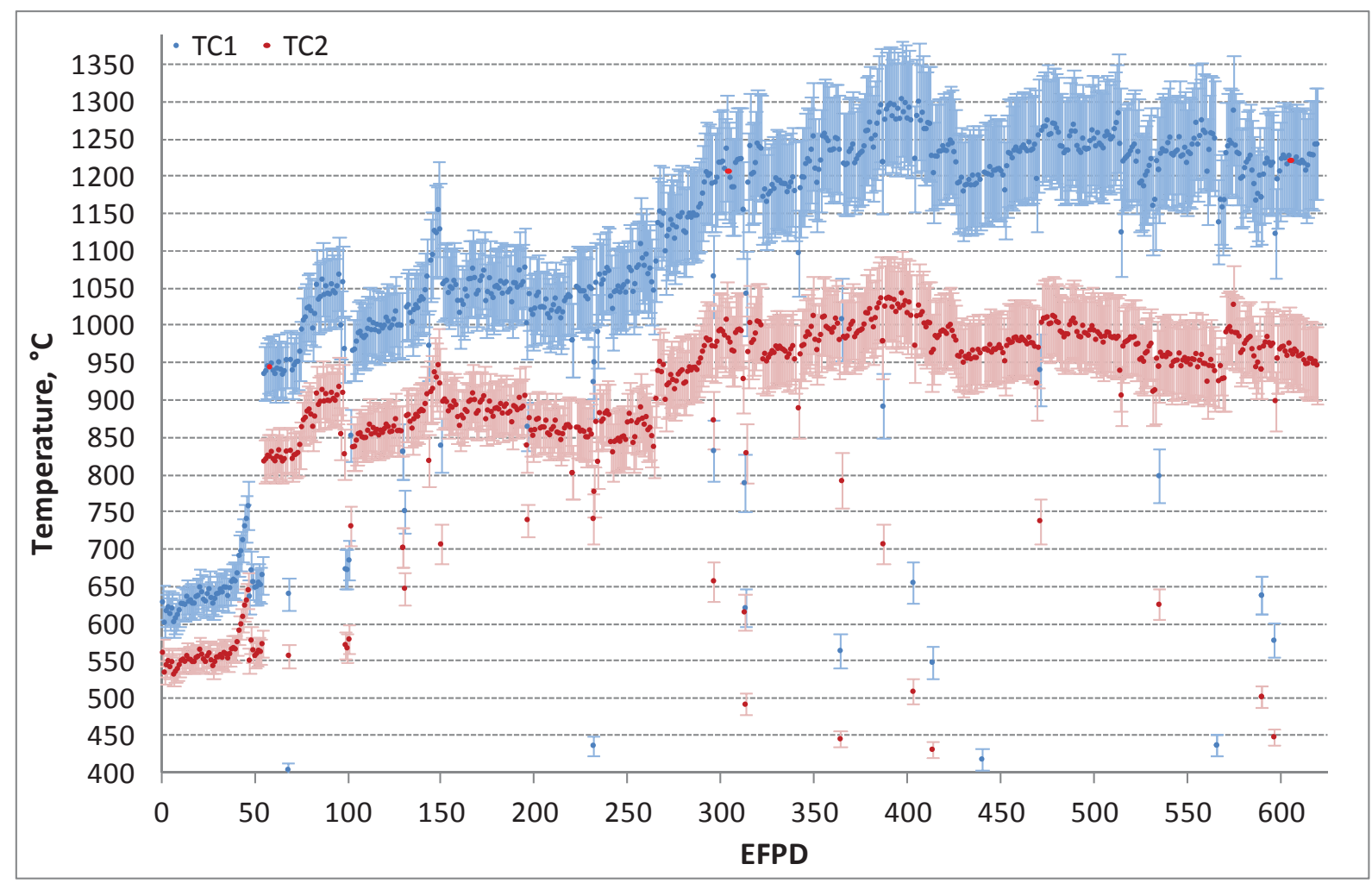

Figure 34. Model temperature and standard deviation of TCs in Capsule 6.

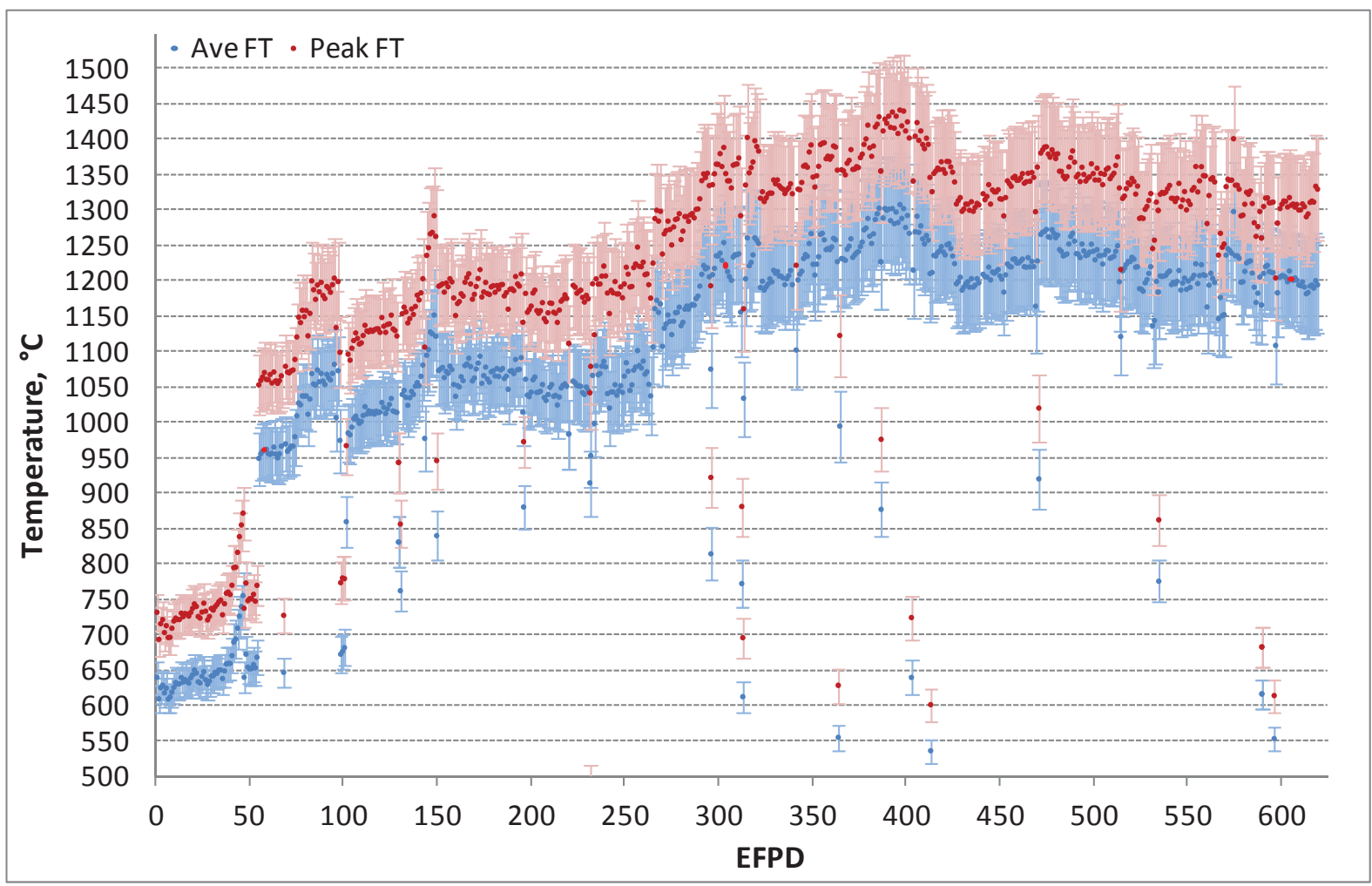

Figure 35. Model temperature and standard deviation of daily averaged fuel temperatures in Capsule 6. 


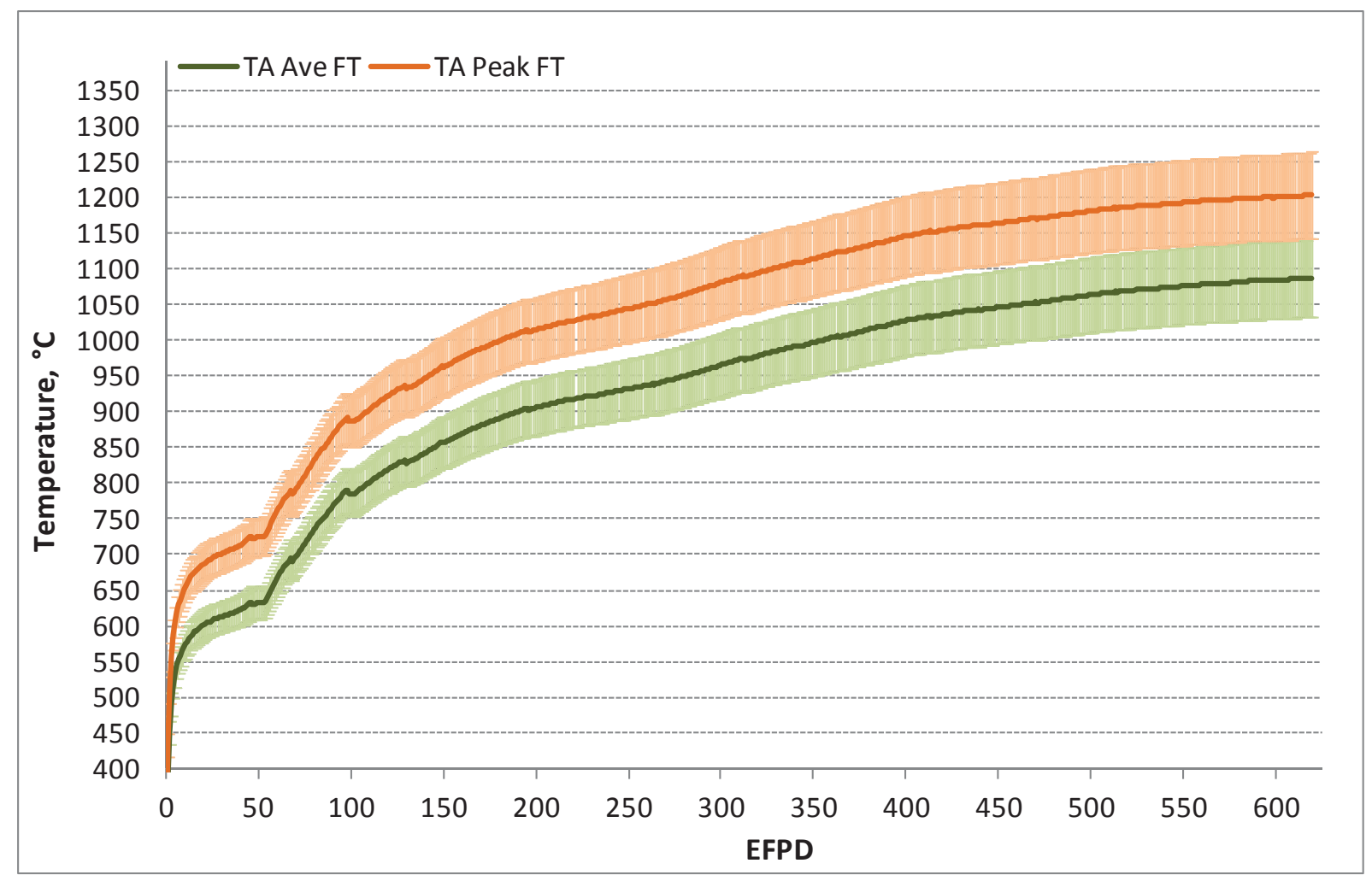

Figure 36. Model temperature and standard deviation of time-average fuel temperatures in Capsule 6.

\subsection{Temperature Uncertainty Results for Capsule 5}

Capsule 5 daily uncertainties (in terms of standard deviations at each time step) are estimated for six calculated temperatures: peripheral TC1, peripheral TC3, daily average VA FT, daily average peak FT, TA VA FT, and TA peak FT. Table 9 summarizes the results for Capsule 5.

Table 9. Summary of temperature uncertainty results for Capsule 5

\begin{tabular}{|l|c|c|c|c|c|}
\hline & $\begin{array}{c}\text { Peripheral } \\
\text { TC1/TC3 }\end{array}$ & VA FT & Peak FT & $\begin{array}{c}\text { TA VA FT } \\
\text { at end }\end{array}$ & $\begin{array}{c}\text { TA Peak FT } \\
\text { at end }\end{array}$ \\
\hline Dominant parameter & Gas gap & Gas gap & $\begin{array}{c}\text { Fuel } \\
\text { conductivity }\end{array}$ & Gas gap & $\begin{array}{c}\text { Fuel } \\
\text { conductivity }\end{array}$ \\
\hline Dominant sensitivity & $0.34-0.38$ & $0.34-0.22$ & $-0.07--0.14$ & & \\
Dominant uncertainty, \% & $7.1-11.0$ & $7.1-11.0$ & 20.0 & & \\
Overall uncertainty, \% & $2.6-4.5$ & $2.7-4.0$ & $2.75-4.7$ & 3.7 & 4.3 \\
Overall uncertainty, ${ }^{\circ} \mathrm{C}$ & $12-35$ & $16-52$ & $20-63$ & 38 & 50 \\
\hline
\end{tabular}

Uncertainty results are displayed as follows:

1. The daily standard deviations for five significant input parameters are presented in Figure 37.

2. The daily input sensitivities of all AGR-1 thermal model time steps for temperatures in Capsule 5 are presented in Figure 38.

3. The daily relative and absolute standard deviations of TC and fuel compact temperature model uncertainty are presented in Figure 39 and Figure 40. 
4. The daily temperature and one standard deviation of TC and fuel compact temperatures in Capsule 5 are presented in Figure 41, Figure 42, and Figure 43.

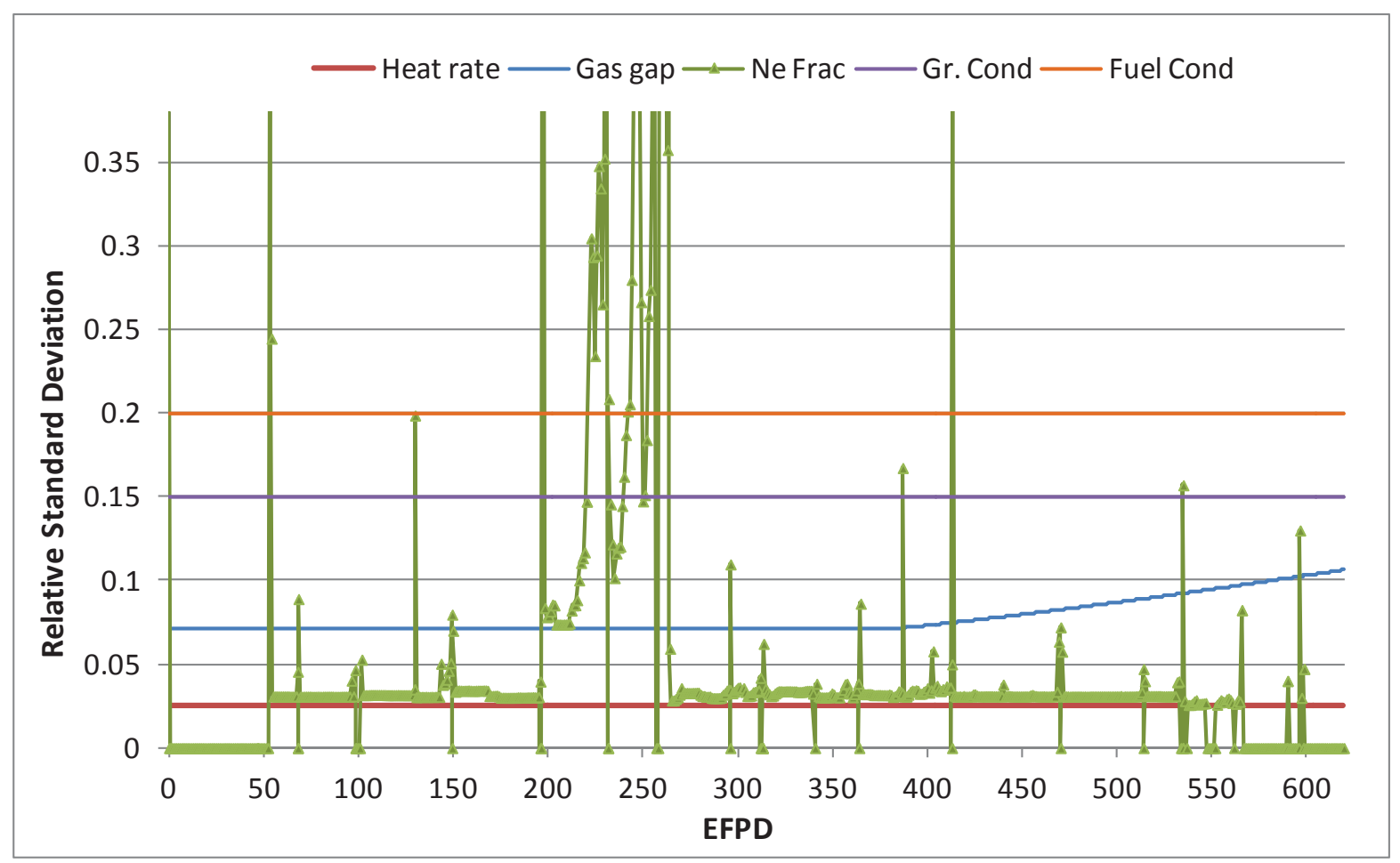

Figure 37. Daily input relative standard deviations for Capsule 5. 

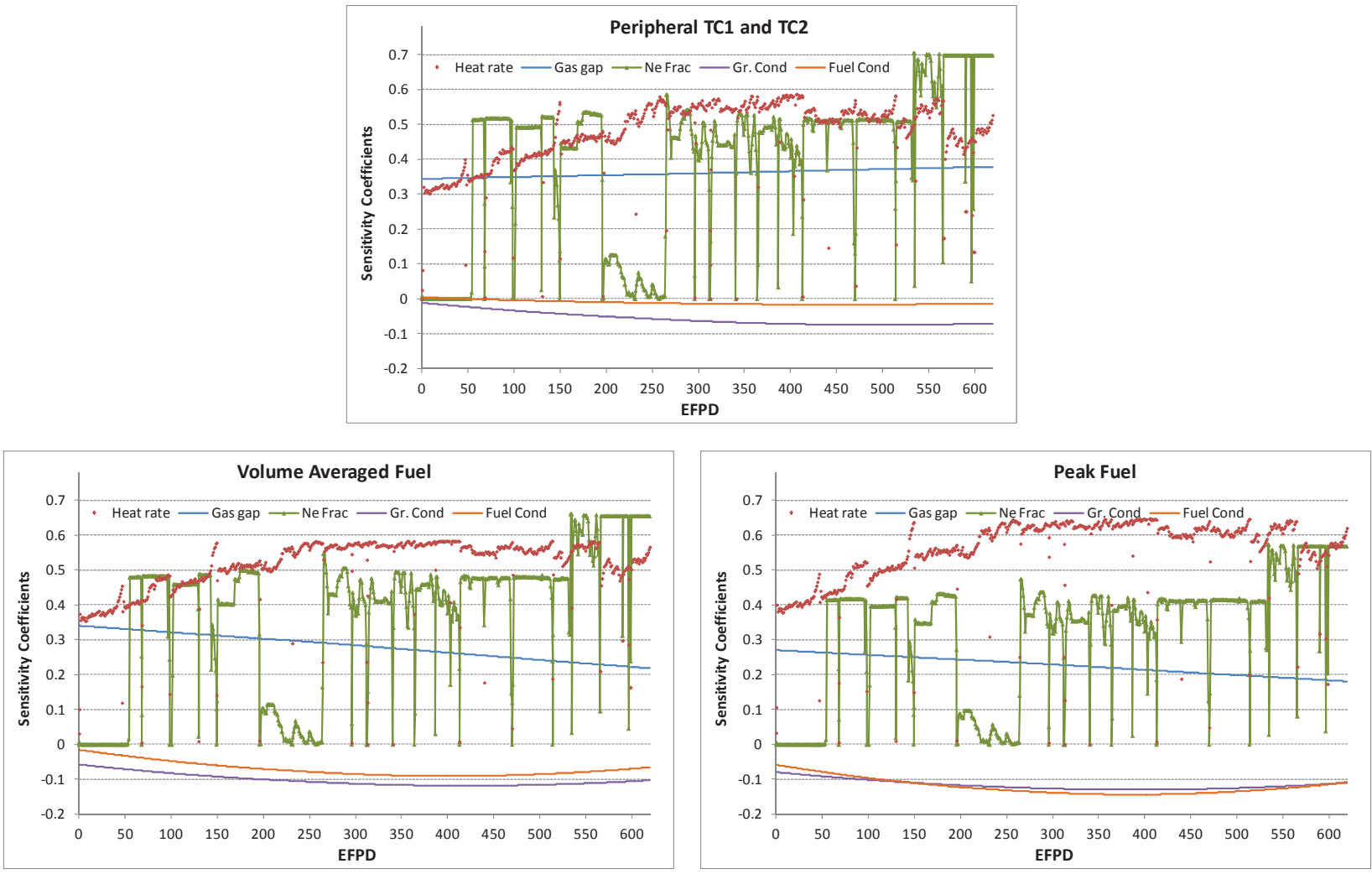

Figure 38. Daily input parameter sensitivities for temperatures in Capsule 5.

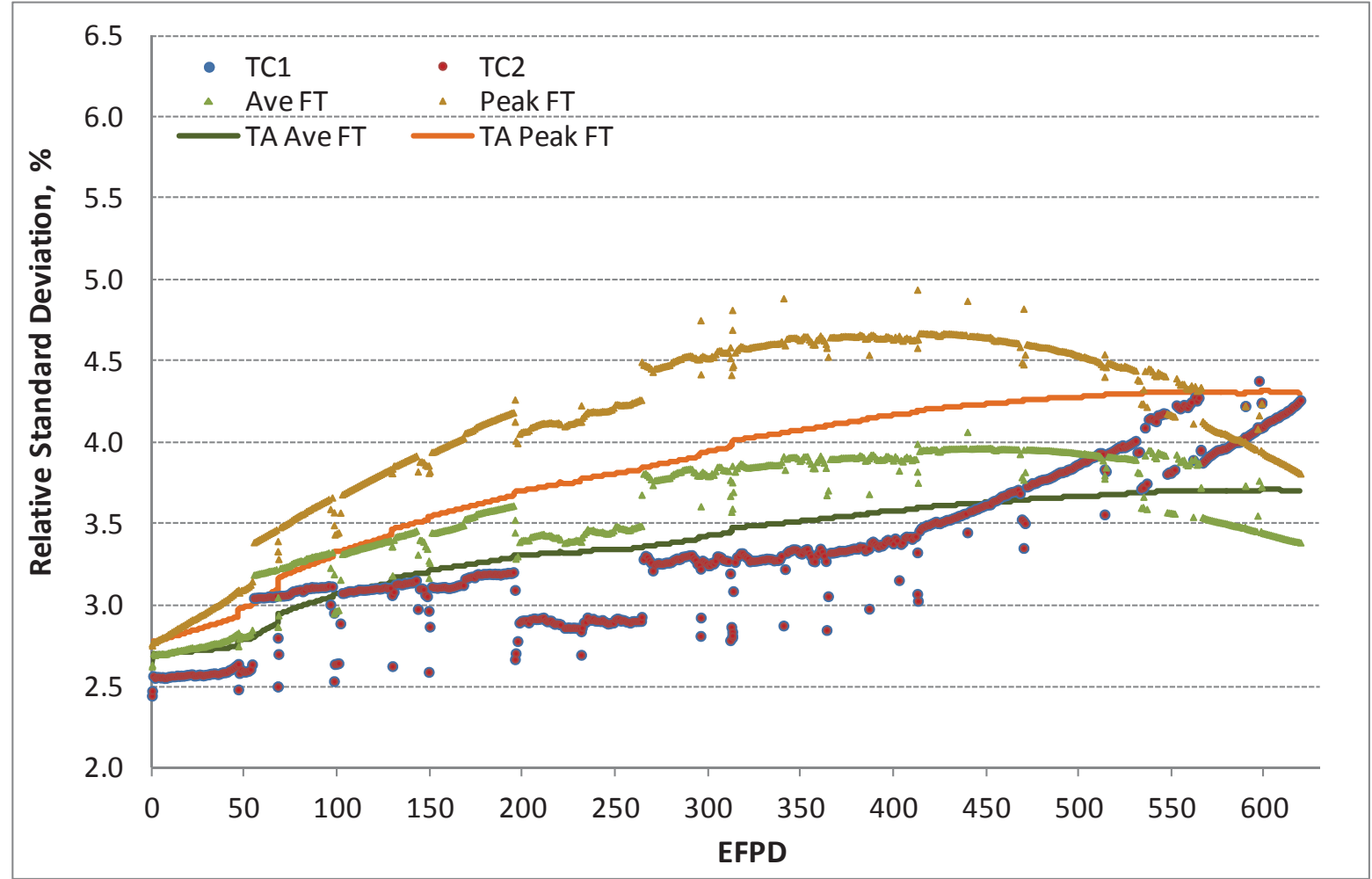

Figure 39. Daily relative standard deviations of predicted TC and fuel temperatures in Capsule 5. 


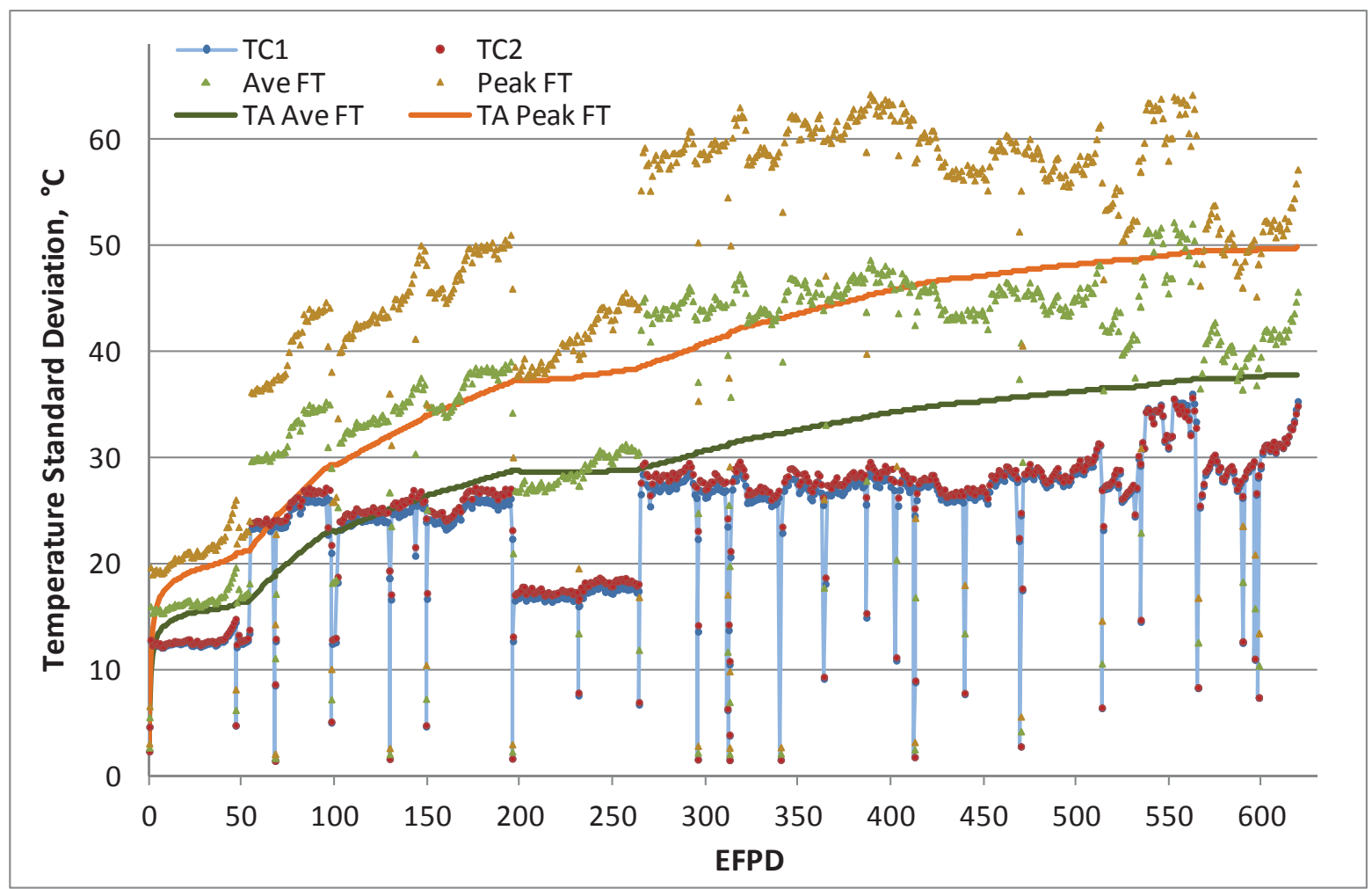

Figure 40. Daily standard deviations of predicted TC and fuel temperatures in Capsule 5.

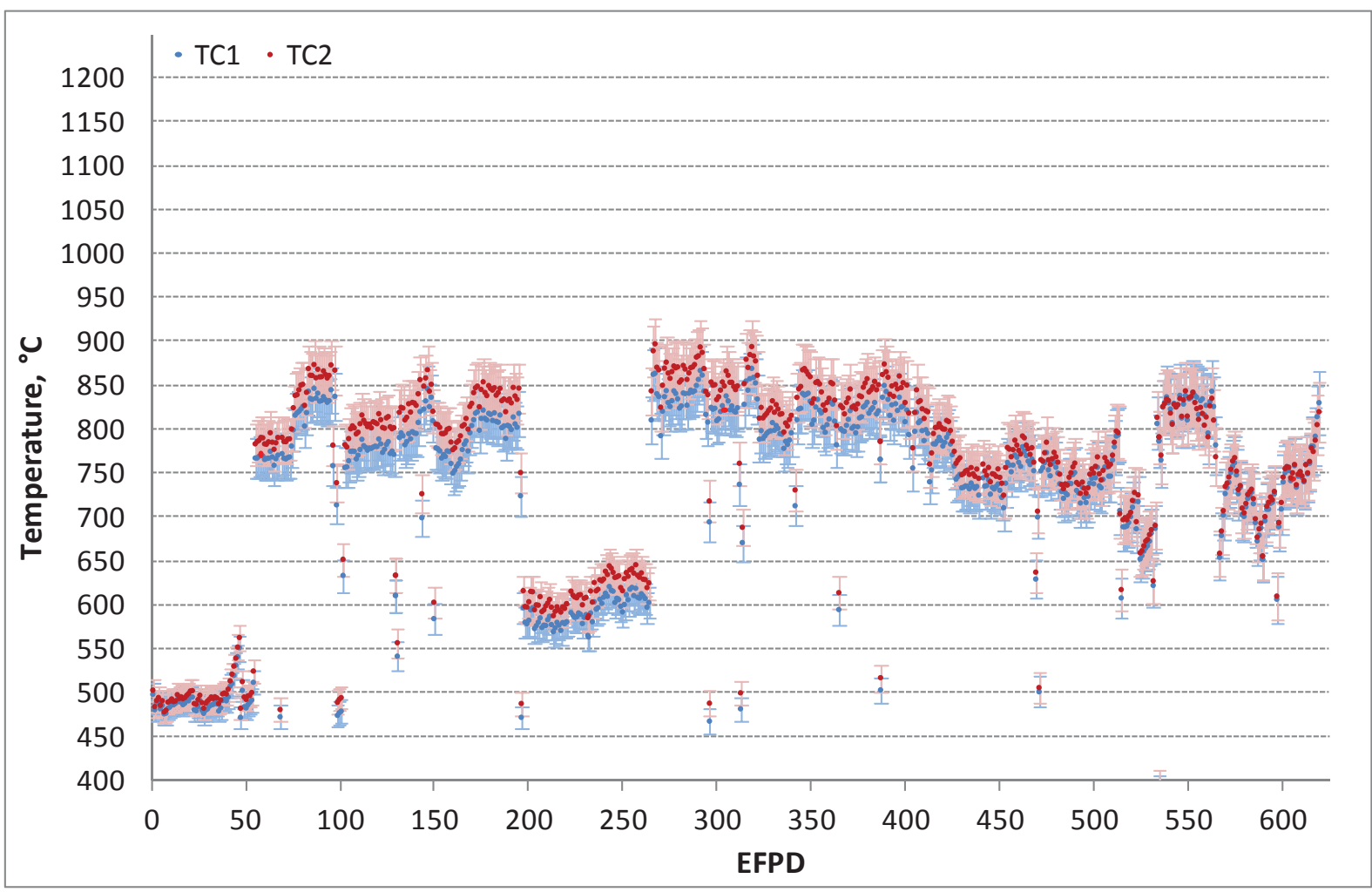

Figure 41. Model temperature and standard deviation of TCs in Capsule 5. 


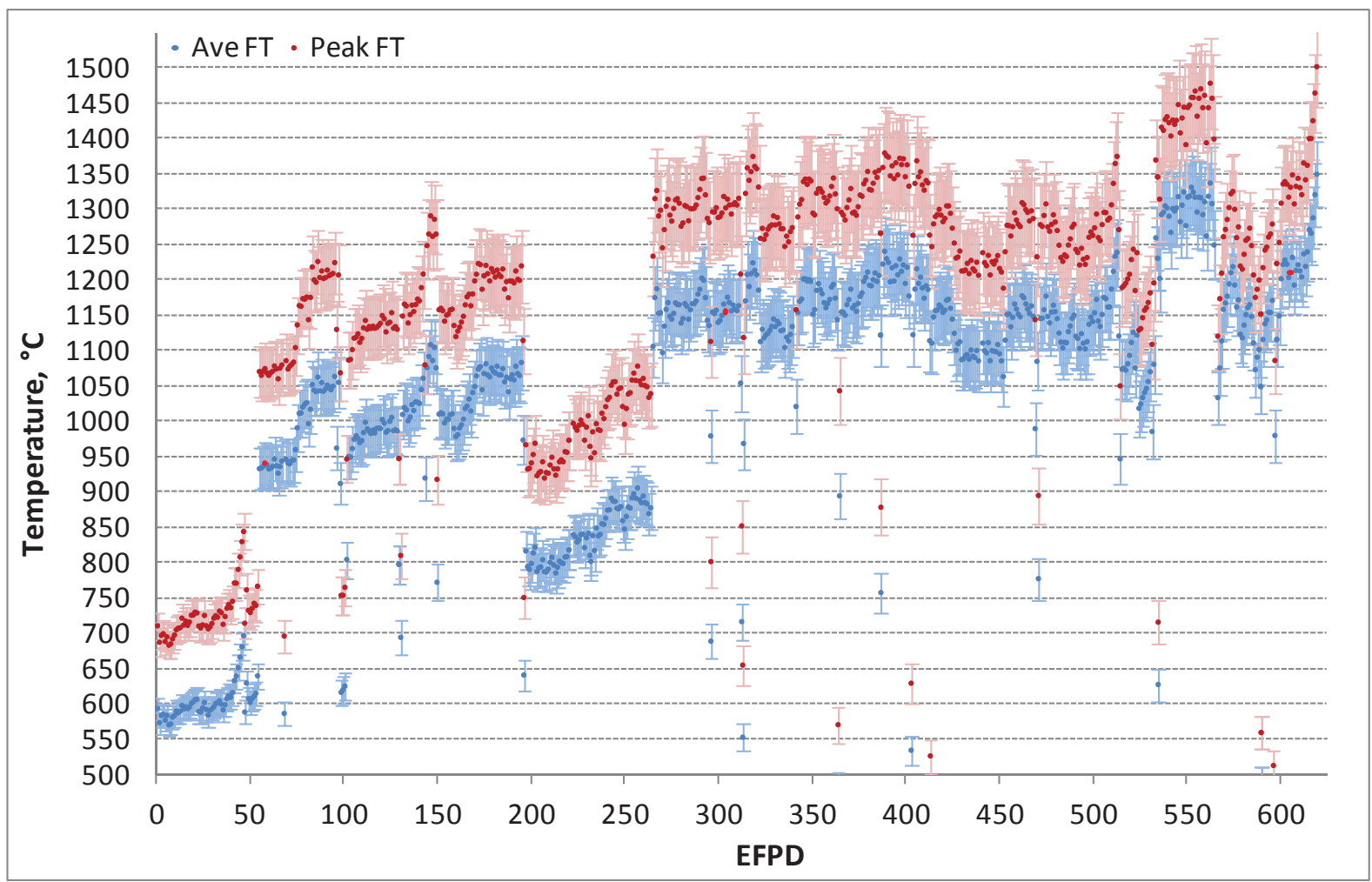

Figure 42. Model temperature and standard deviation of daily fuel temperatures in Capsule 5.

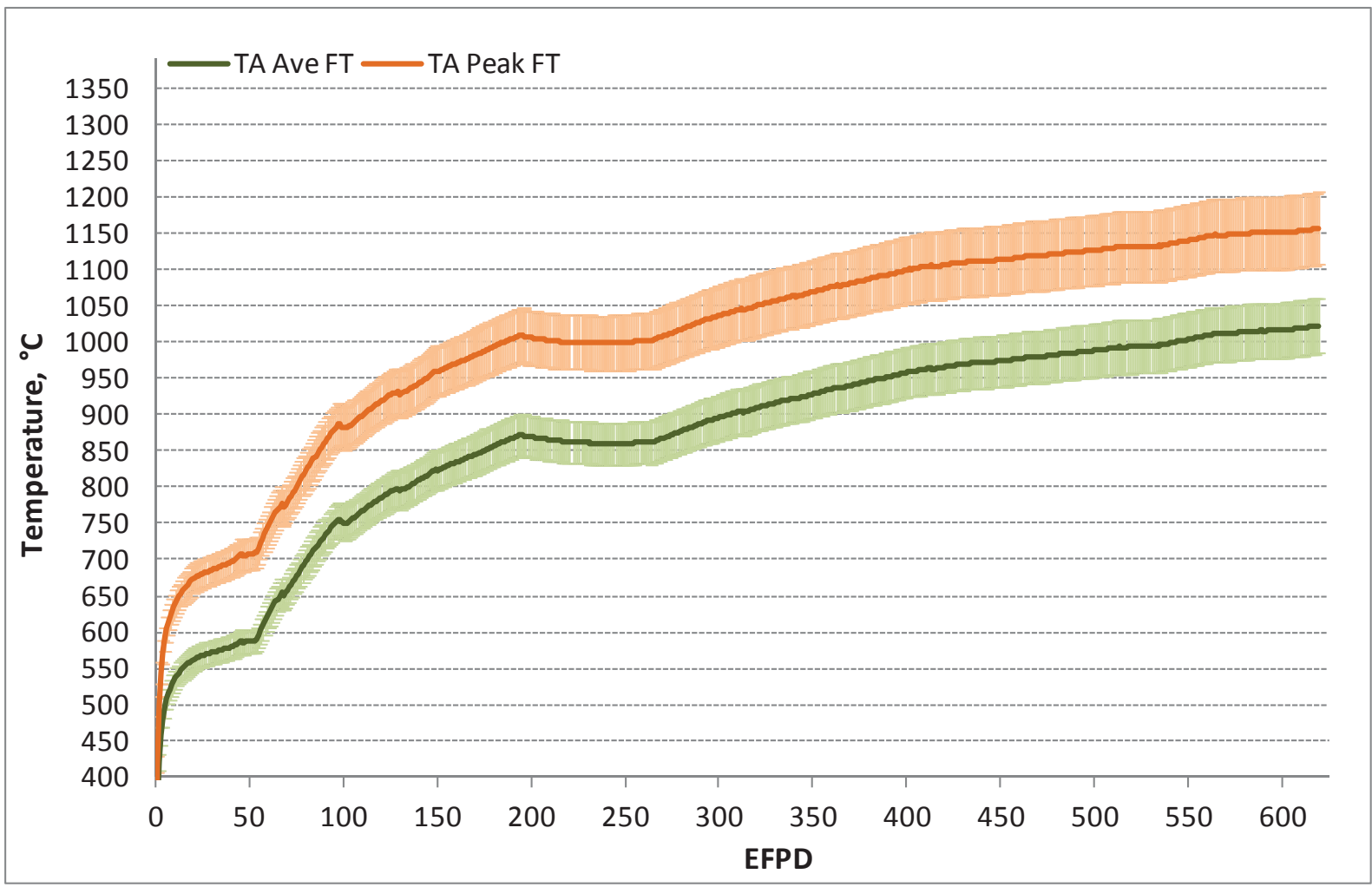

Figure 43. Model temperature and standard deviation of time-average fuel temperatures in Capsule 5. 


\subsection{Temperature Uncertainty Results for Capsule 4}

Capsule 4 daily uncertainties (in terms of standard deviations at each time step) are estimated for six calculated temperatures: peripheral TC1, peripheral TC3, daily average VA FT, daily average peak FT, TA VA FT, and TA peak FT. Table 10 summarizes the results for Capsule 4.

Table 10. Summary of temperature uncertainty results for Capsule 4

\begin{tabular}{|lcccc|c|c|c|}
\hline & Center TC2 & $\begin{array}{c}\text { Peripheral } \\
\text { TC1 }\end{array}$ & VA FT & Peak FT & $\begin{array}{c}\text { TA VA } \\
\text { FT at end }\end{array}$ & $\begin{array}{c}\text { TA Peak } \\
\text { FT at end }\end{array}$ \\
\hline Dominant parameter & $\begin{array}{l}\text { Graphite } \\
\text { conductivity }\end{array}$ & Gas gap & Gas gap & $\begin{array}{l}\text { Fuel } \\
\text { conductivity }\end{array}$ & Gas gap & $\begin{array}{l}\text { Fuel } \\
\text { conductivity }\end{array}$ \\
\hline Dominant sensitivity & $-0.10--0.21$ & $0.38-0.40$ & $0.25-0.16$ & $-0.08--0.15$ & & \\
Dominant uncertainty, \% & 15 & $9.7-14.5$ & $9.7-14.5$ & 20 & & 4.3 \\
Overall uncertainty, \% & $3.1-5.1$ & $3.7-5.9$ & $2.8-4.0$ & $2.8-4.7$ & 3.7 & 52 \\
Overall uncertainty, ${ }^{\circ} \mathrm{C}$ & $18-63$ & $17-45$ & $17-50$ & $20-65$ & 40 & 52 \\
\hline
\end{tabular}

Since all functional relationships are constructed using data in Capsule 4, the uncertainty quantification procedure is applied without any capsule specific corrections. Results are displayed as follows:

1. The daily standard deviations of five significant input parameters in Capsule 4 are presented in Figure 44.

2. The daily input sensitivities of all AGR-1 thermal model time steps for temperatures in Capsule 4 are presented in Figure 45.

3. The daily relative and absolute standard deviations of TC and fuel compact temperature model uncertainties are presented in Figure 46 and Figure 47.

4. The daily temperature and one standard deviation of TC and fuel compact temperatures in Capsule 4 are presented in Figure 48, Figure 49, and Figure 50. 


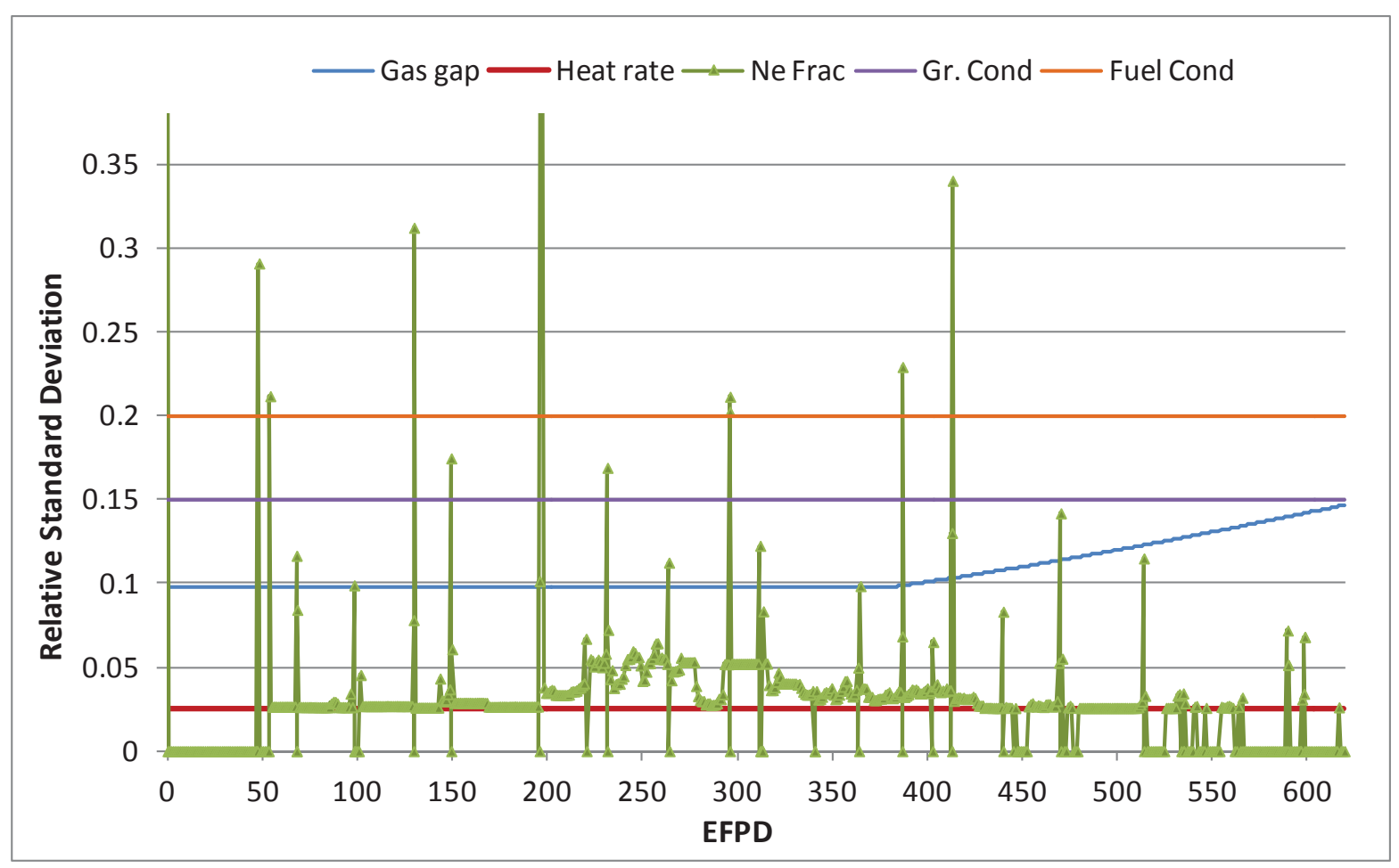

Figure 44. Daily input relative standard deviations for Capsule 4.
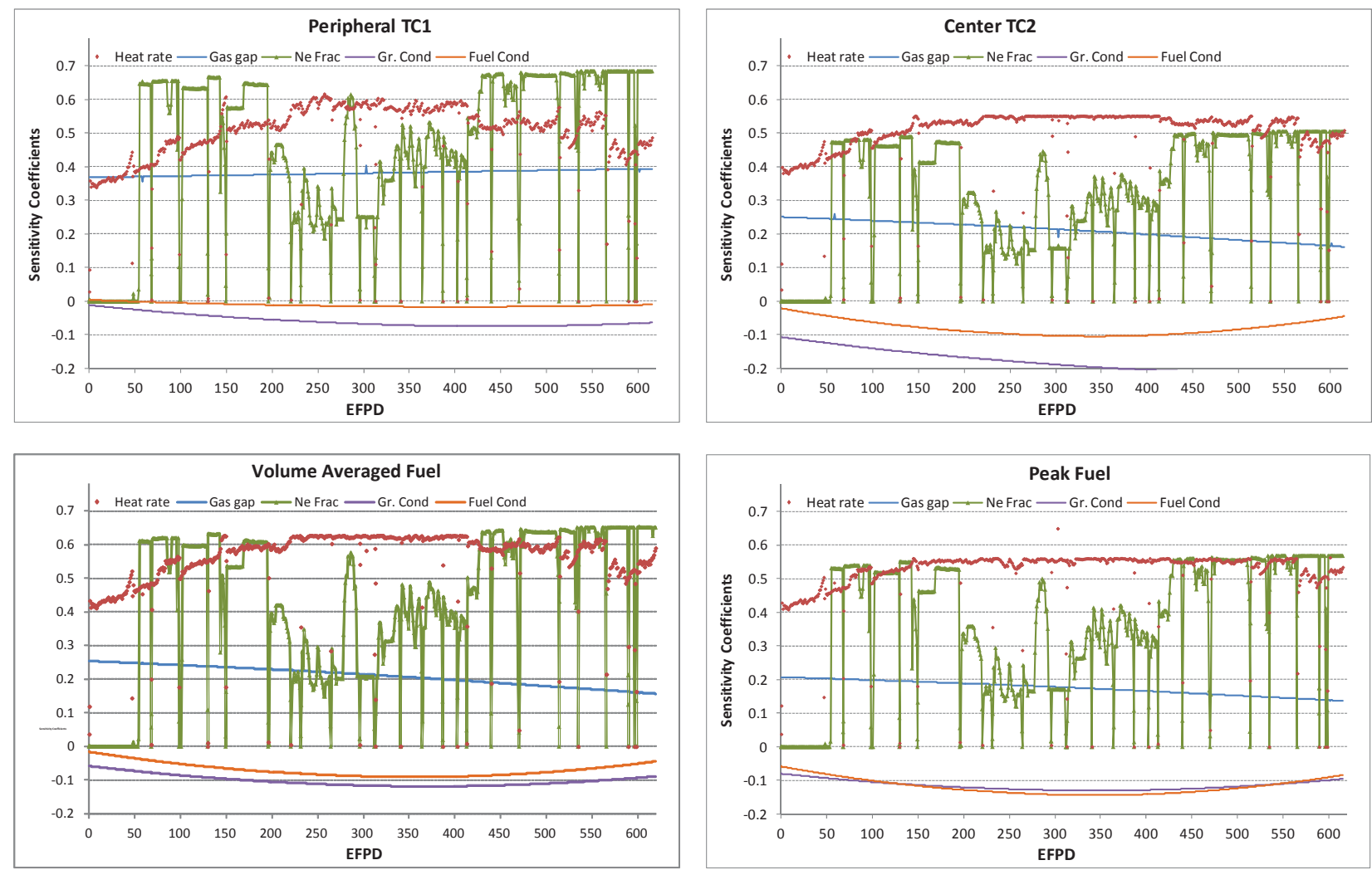

Figure 45. Daily input parameter sensitivities for temperatures in Capsule 4. 


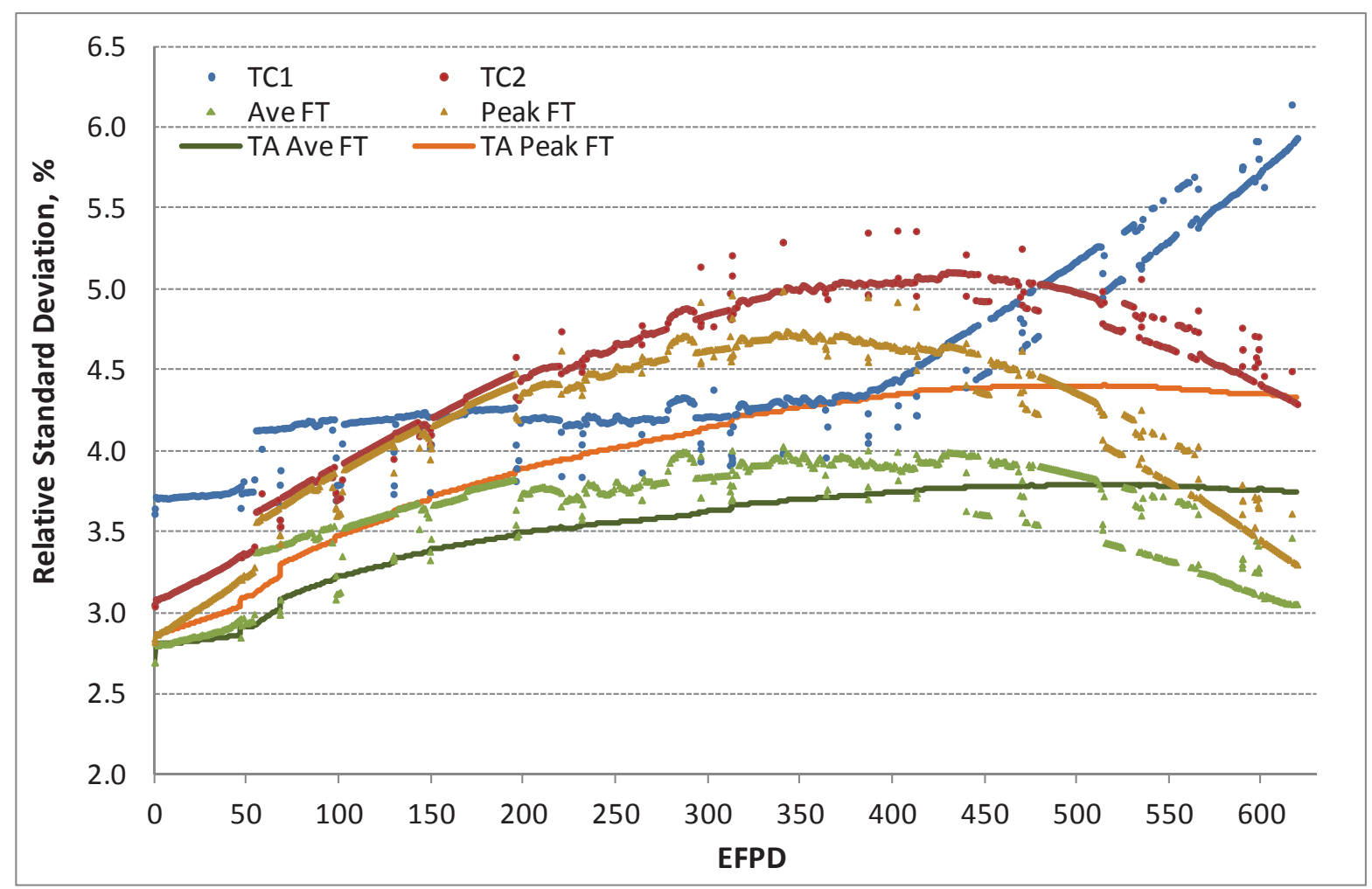

Figure 46. Capsule 4 calculated TC and fuel temperature (FT) relative standard deviations.

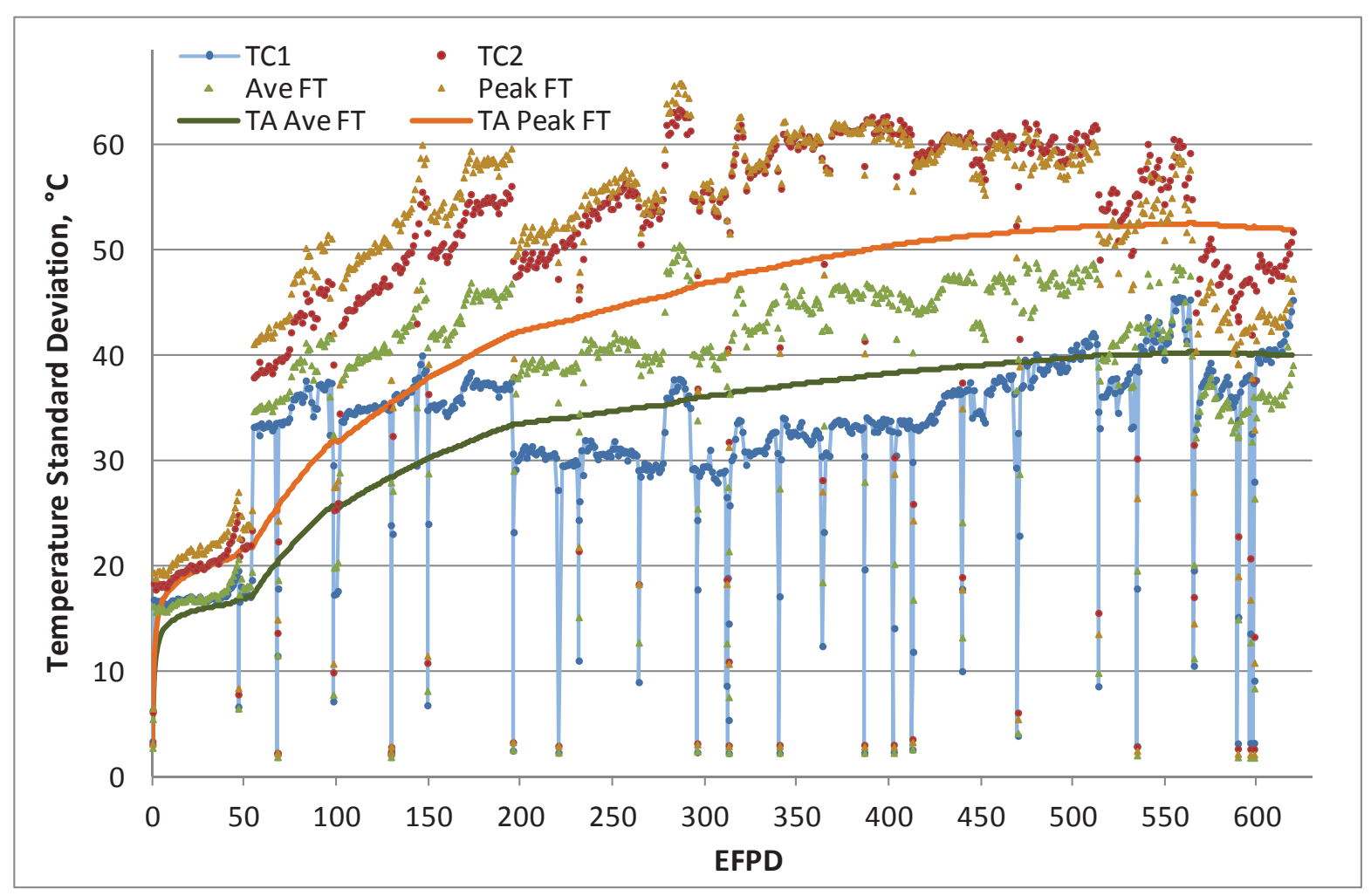

Figure 47. Capsule 4 calculated TC and fuel temperature (FT) standard deviations. 


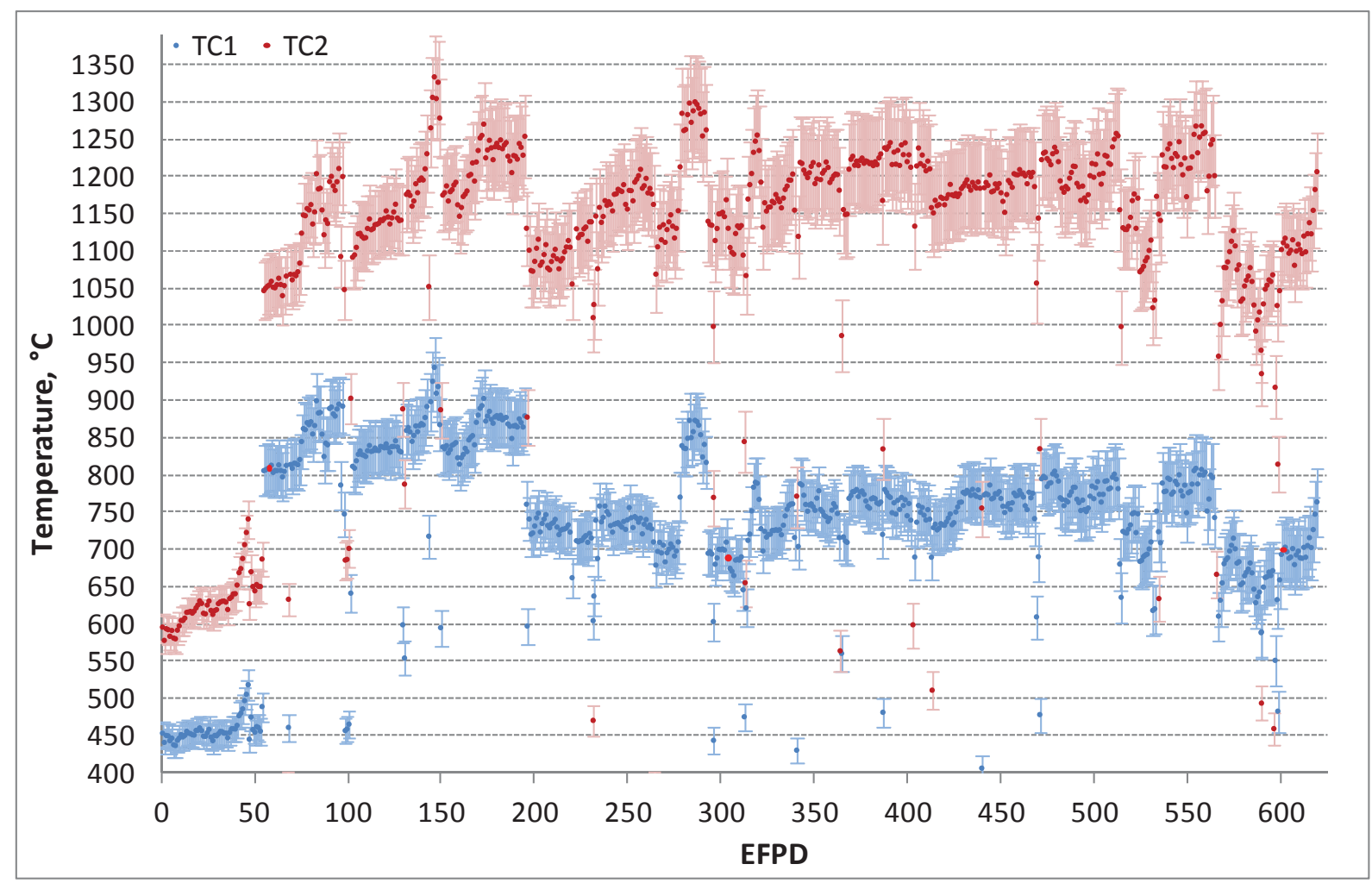

Figure 48. Model temperature and standard deviation of TCs in Capsule 4.

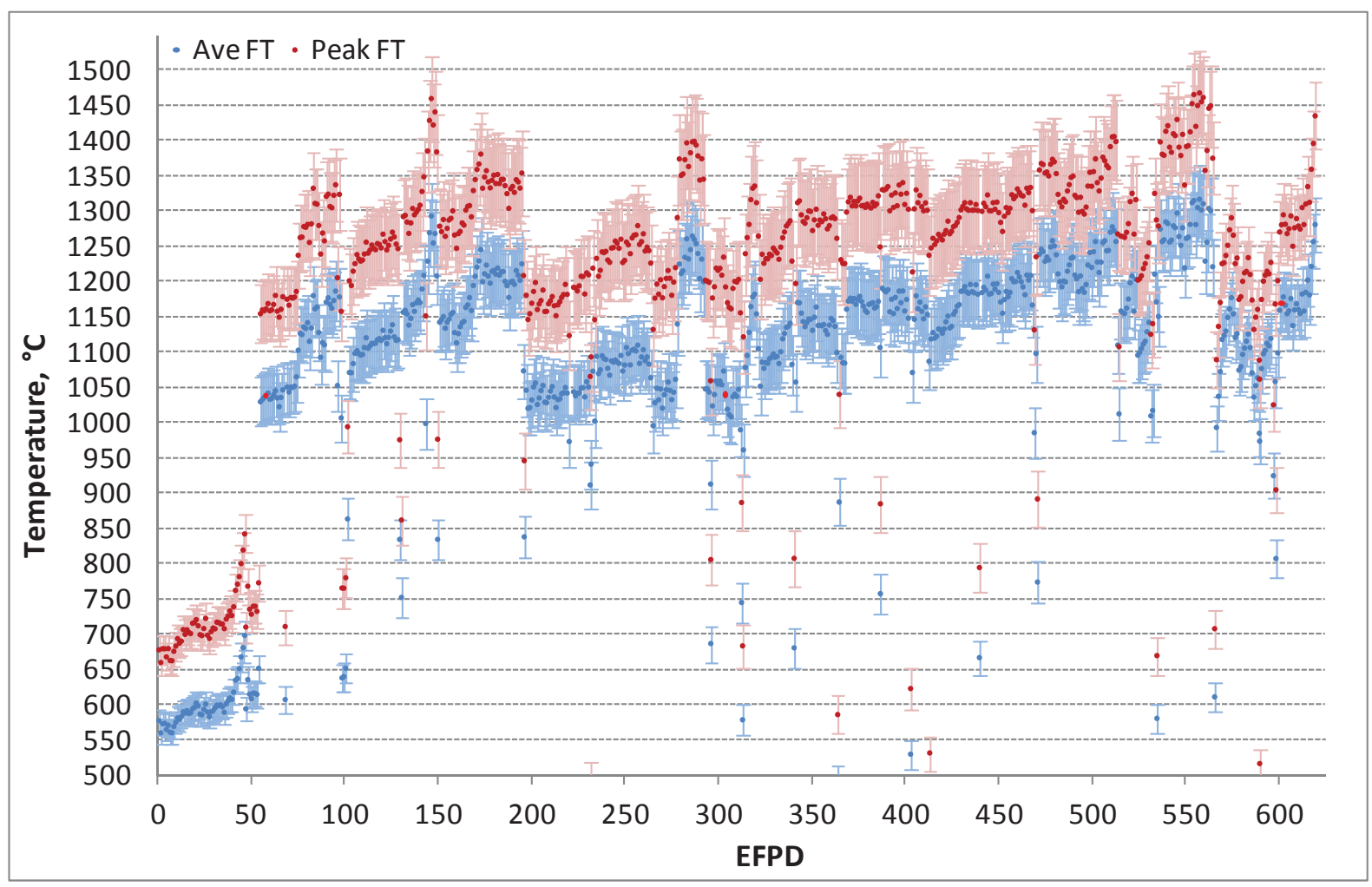

Figure 49. Model temperature and standard deviation of fuel compacts in Capsule 4. 


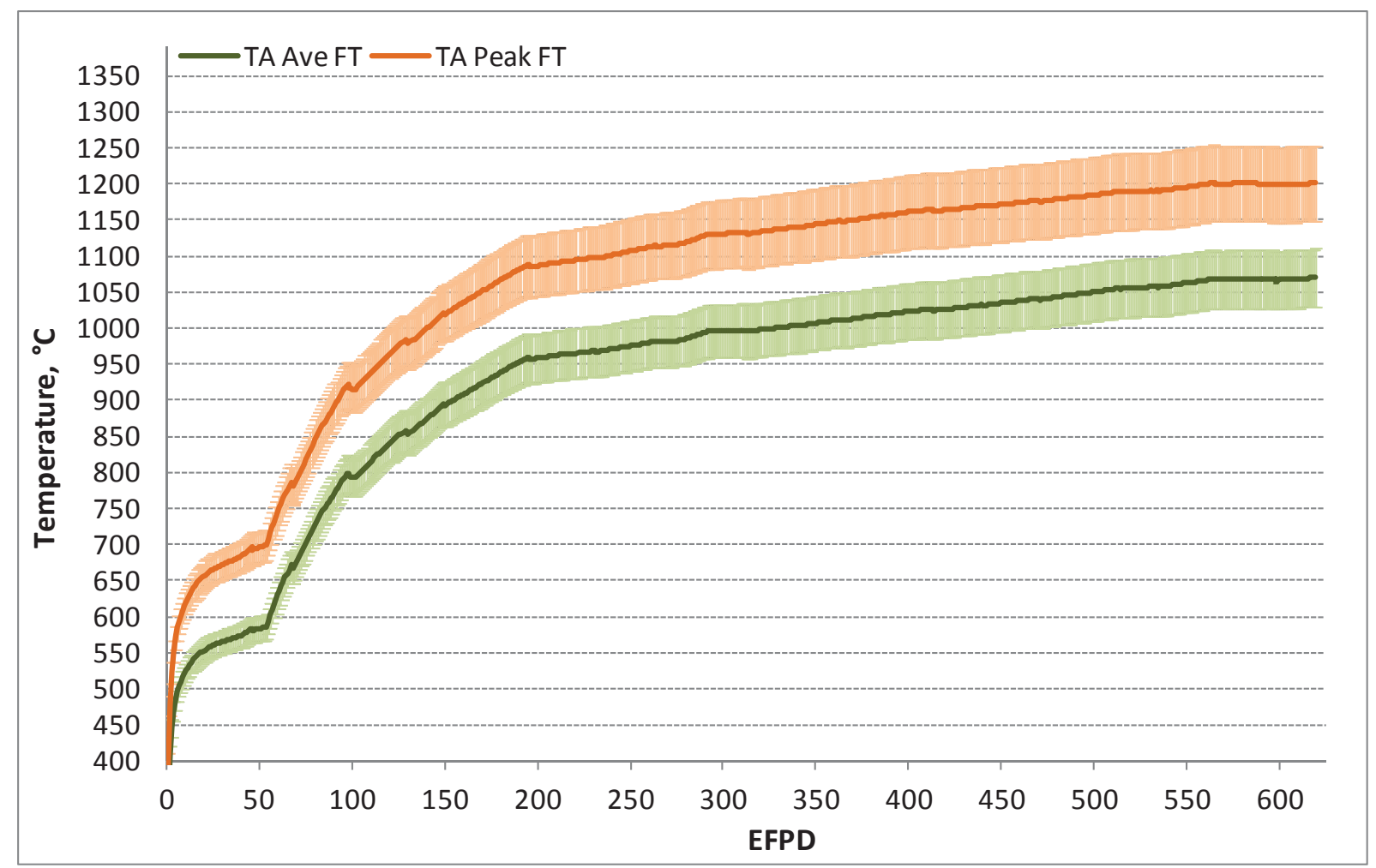

Figure 50. Model temperature and standard deviation of time-average fuel temperatures in Capsule 4.

\subsection{Temperature Uncertainty Results for Capsule 3}

Capsule 3 daily uncertainties (in terms of standard deviations at each time step) are estimated for six calculated temperatures: peripheral TC1, peripheral TC3, daily average VA FT, daily average peak FT, TA VA FT, and TA peak FT. Table 11 summarizes the results for Capsule 3.

Table 11. Summary of temperature uncertainty results for Capsule 3

\begin{tabular}{|l|c|c|c|c|c|}
\hline & $\begin{array}{c}\text { Peripheral } \\
\text { TC1/TC2 }\end{array}$ & VA FT & Peak FT & $\begin{array}{c}\text { TA VA FT } \\
\text { at end }\end{array}$ & $\begin{array}{c}\text { TA Peak FT at } \\
\text { end }\end{array}$ \\
\hline Dominant parameter & Gas gap & Gas gap & Fuel conductivity & Gas gap & Fuel heat rate \\
\hline Dominant sensitivity & $0.38-0.40$ & $0.24-0.15$ & $-0.08--0.16$ & & \\
Dominant uncertainty, $\%$ & $10.7-16.0$ & $10.7-16.0$ & 20 & & \\
Overall uncertainty, $\%$ & $4.1-6.5$ & $2.9-4.0$ & $2.9-4.7$ & 3.8 & 4.3 \\
Overall uncertainty, ${ }^{\circ} \mathrm{C}$ & $17-48$ & $17-48$ & $20-60$ & 39 & 50 \\
\hline
\end{tabular}

Since irradiation thermal conditions in Capsule 3 are similar to Capsule 4, the uncertainty quantification procedure is applied for Capsule 3 without any specific corrections. Results are displayed as follows:

1. The daily standard deviations of five significant input parameters for Capsule 3 are presented in Figure 51.

2. The daily input sensitivities of all AGR-1 time steps for temperatures in Capsule 3 are plotted in Figure 52. 
3. The daily relative and absolute standard deviations of TC and fuel compact temperature model uncertainty are presented in Figure 53 and Figure 54. The model temperature uncertainty for peripheral TC1 and TC2 in Capsule 3 (Figure 53) reached $6.5 \%$ by the end of the AGR-1 test because of high gap distance standard deviation $(16 \%)$ and high gap distance sensitivity $(\sim 0.4)$.

4. The daily temperature and one standard deviation of the predicted temperatures in Capsule 3 are plotted for: central TC1 and peripheral TC2 in Figure 55, daily average VA FT and daily peak FT in Figure 56, and TA VA FT and TA peak FT in Figure 57.

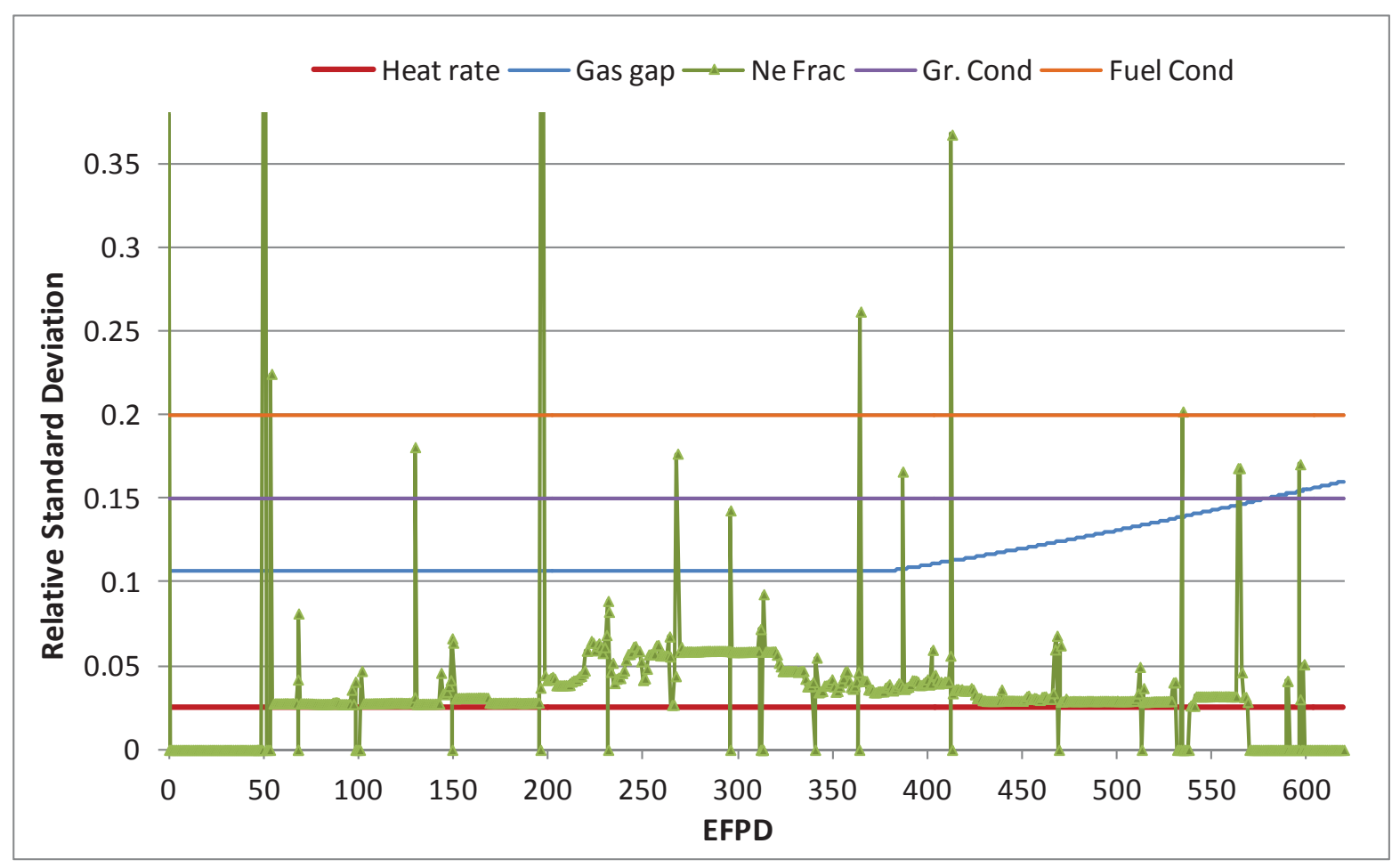

Figure 51. Daily input relative standard deviations for Capsule 3. 

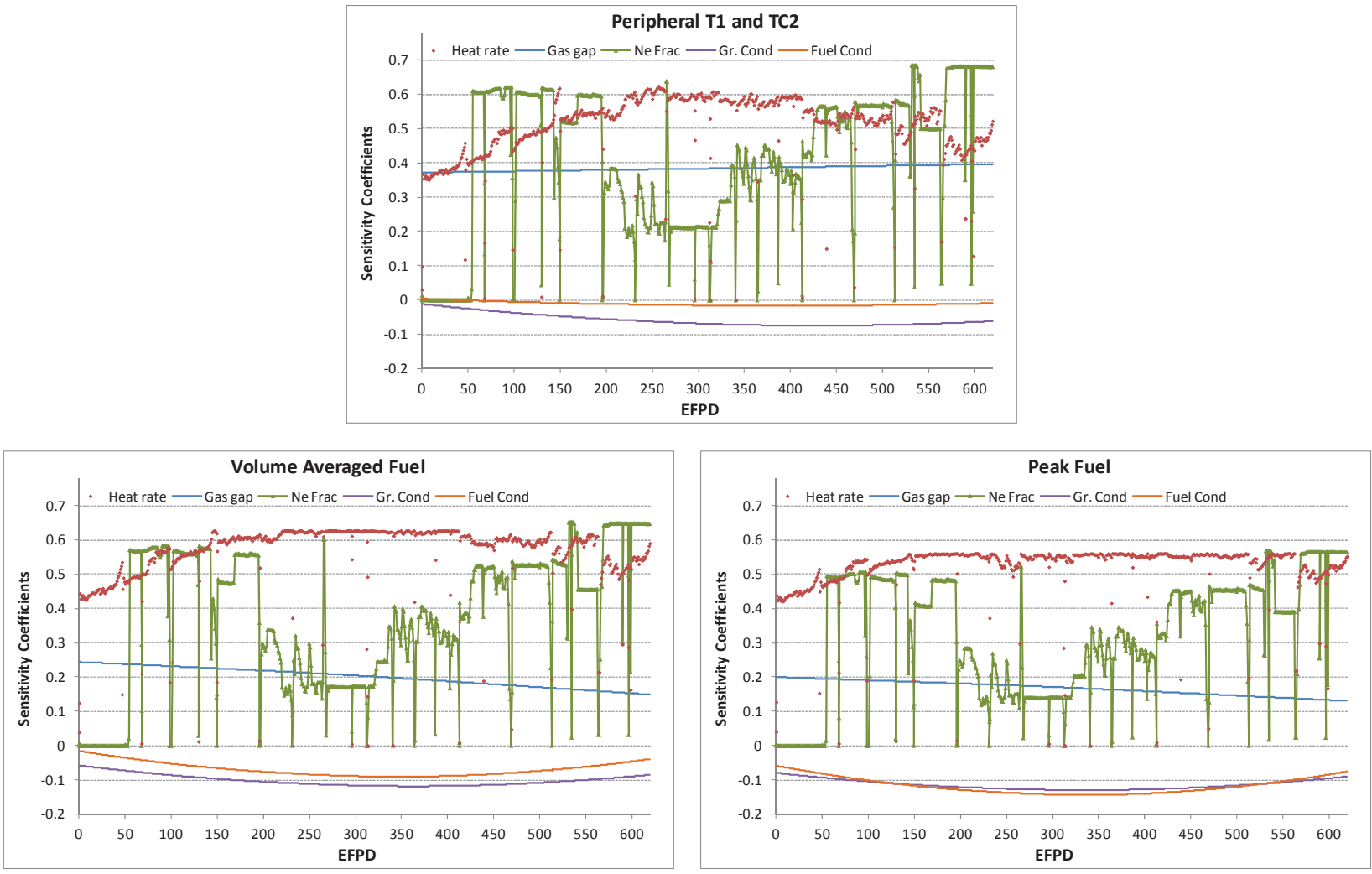

Figure 52. Daily input parameter sensitivities for temperatures in Capsule 3.

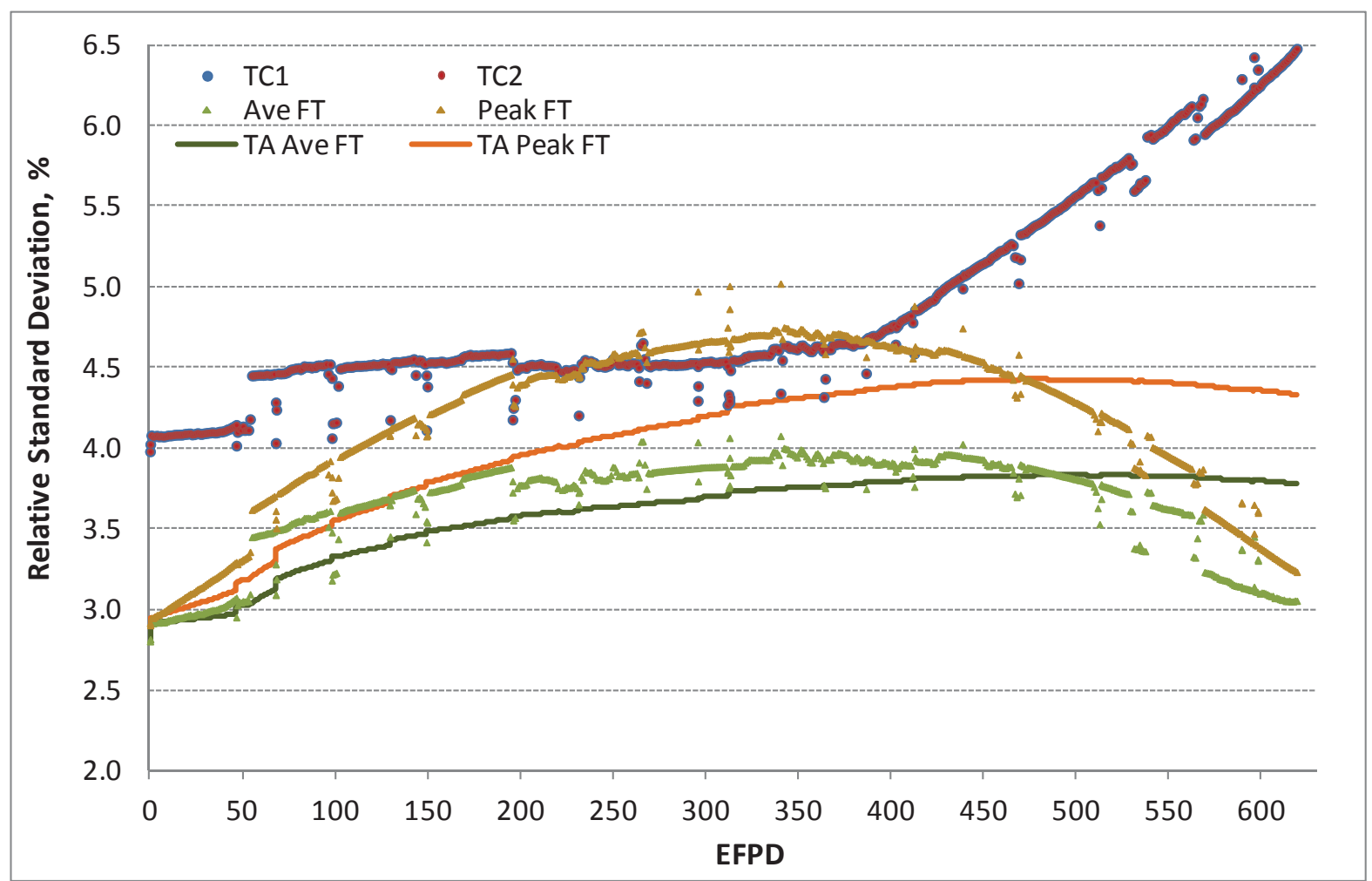

Figure 53. Daily relative standard deviations of predicted TC and fuel temperatures in Capsule 3. 


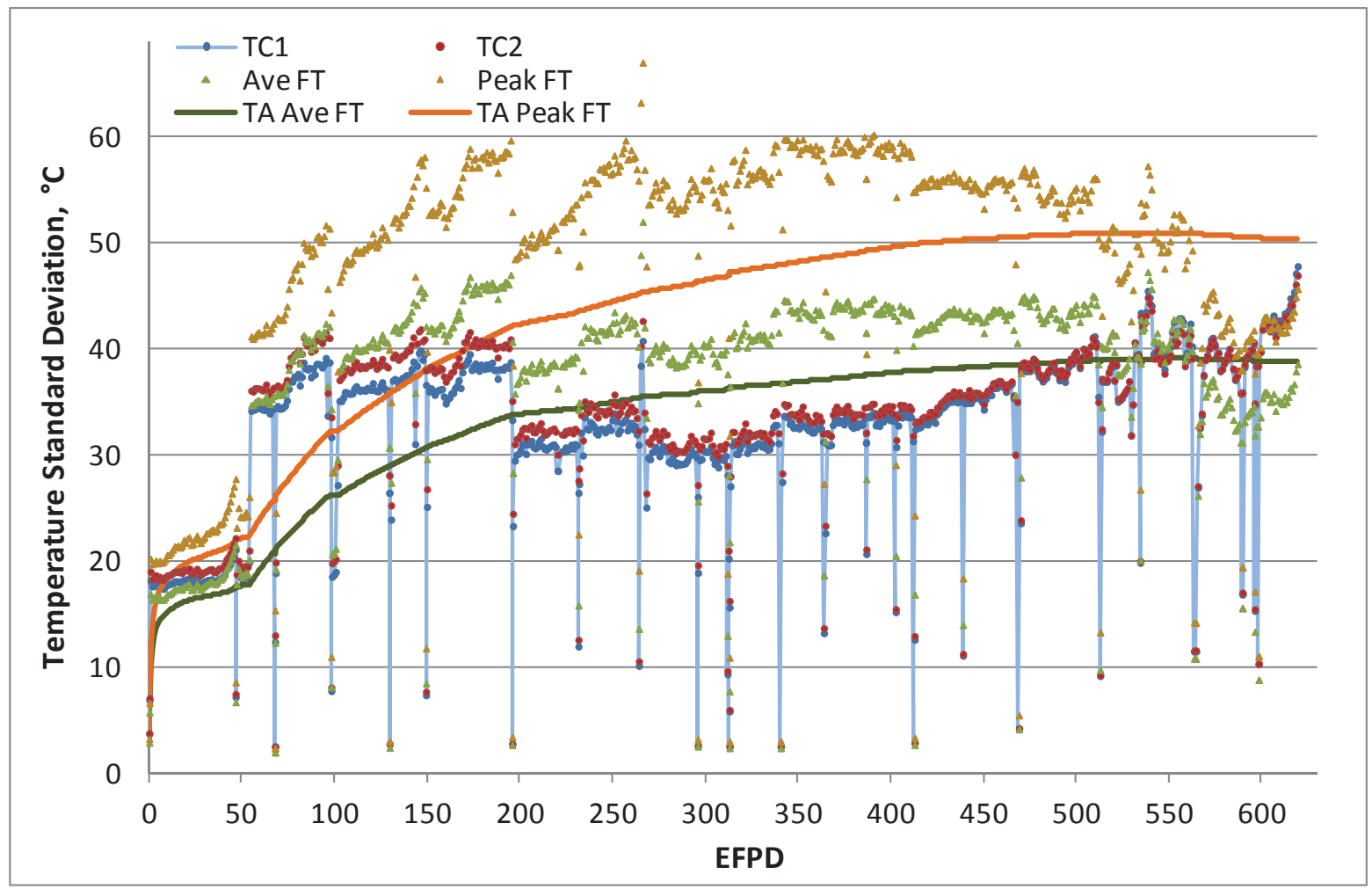

Figure 54. Daily standard deviations of predicted TC and fuel temperatures in Capsule 3.

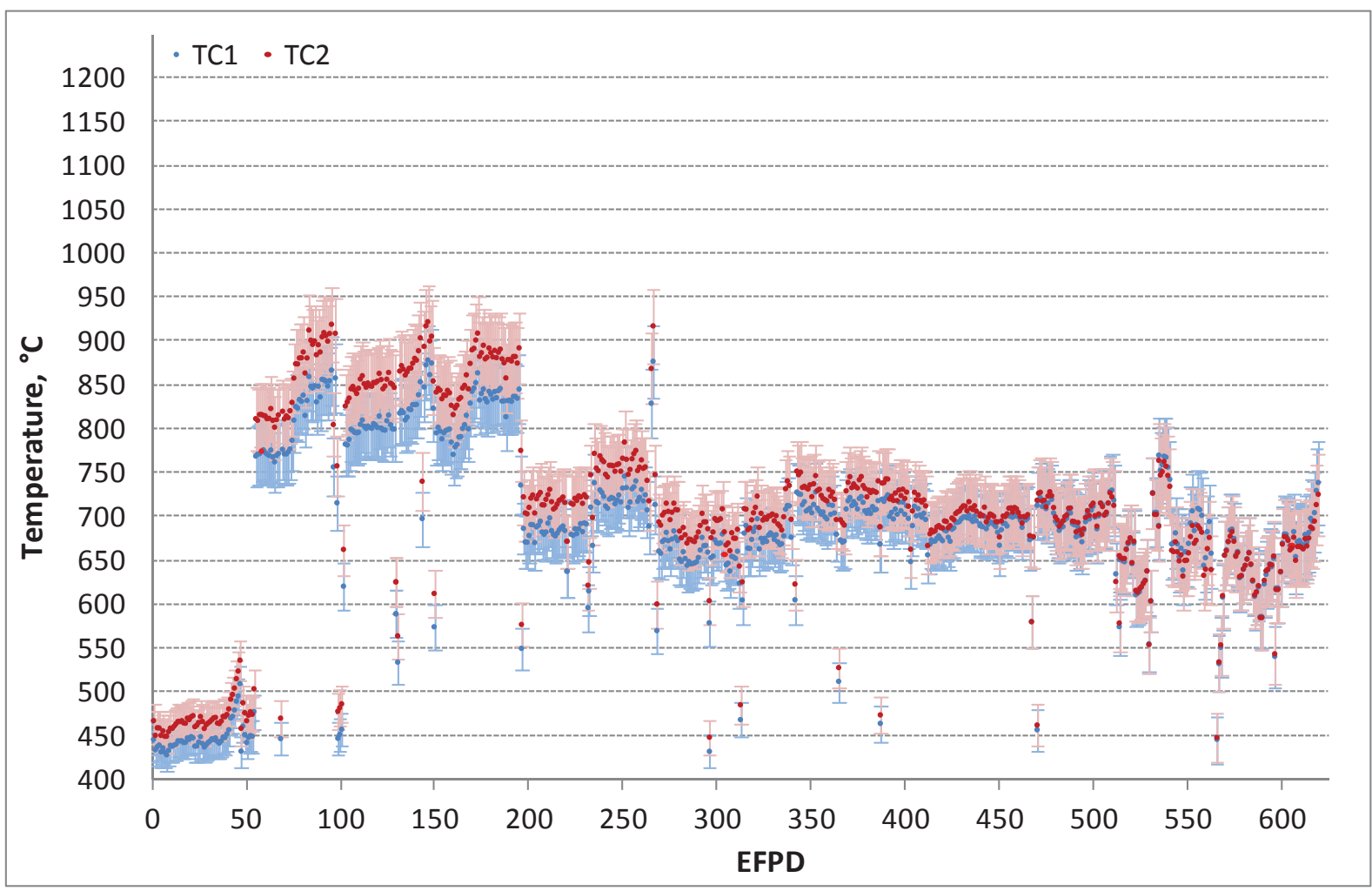

Figure 55. Model temperature and standard deviation of TCs in Capsule 3. 


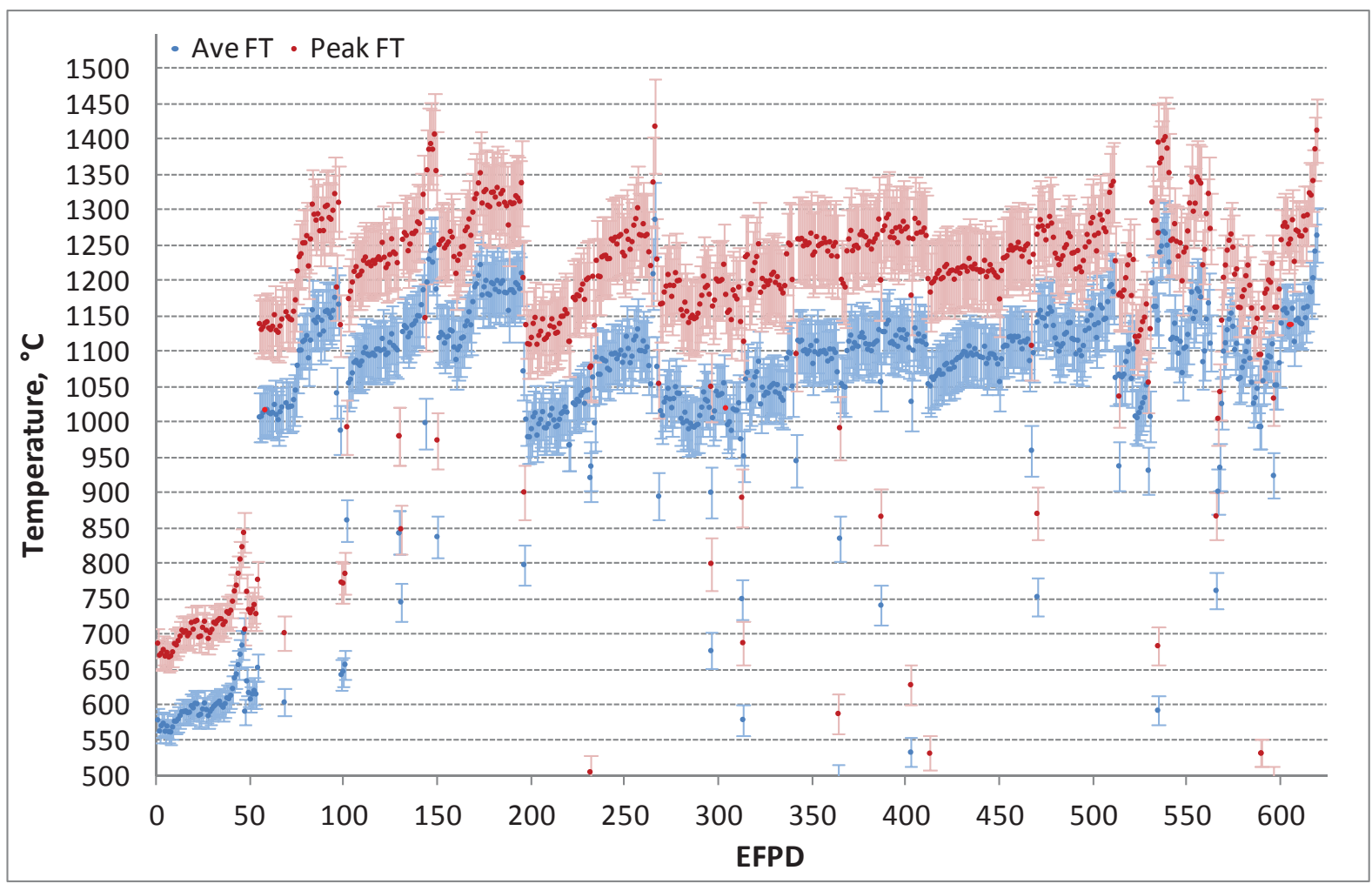

Figure 56. Model temperature and standard deviation of daily fuel temperatures in Capsule 3.

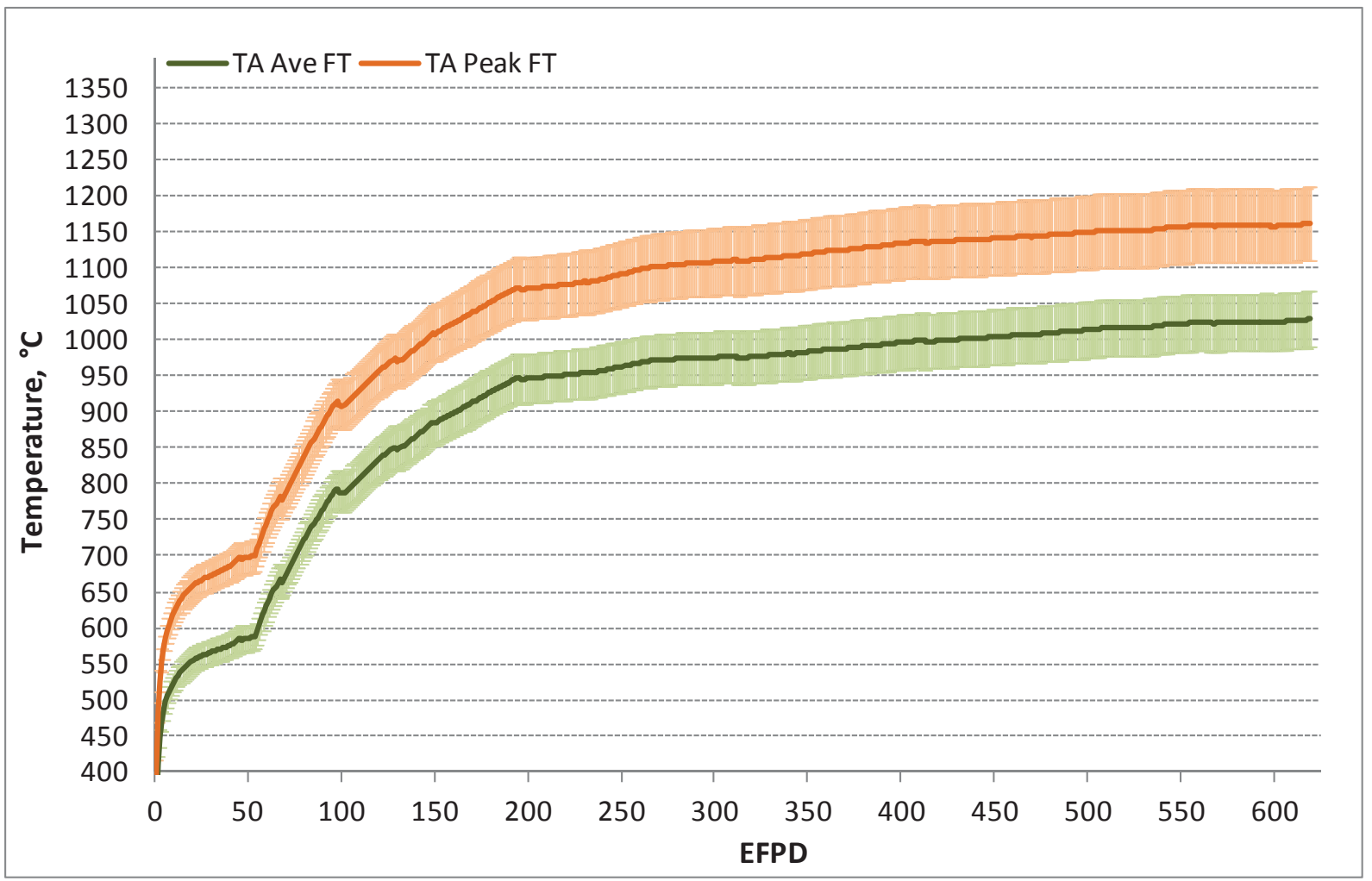

Figure 57. Model temperature and standard deviation of time-average fuel temperatures in Capsule 3. 


\subsection{Temperature Uncertainty Results for Capsule 2}

Capsule 2 daily uncertainties (in terms of standard deviations at each time step) are estimated for six calculated temperatures: peripheral TC1, peripheral TC3, daily average VA FT, daily average peak FT, TA VA FT, and TA peak FT. Table 12 summarizes the results for Capsule 2.

Table 12. Summary of temperature uncertainty results for Capsule 2

\begin{tabular}{|c|c|c|c|c|c|}
\hline & $\begin{array}{l}\text { Peripheral } \\
\text { TC1/TC2 }\end{array}$ & VA FT & Peak FT & $\begin{array}{l}\text { TA VA FT } \\
\text { at end }\end{array}$ & $\begin{array}{l}\text { TA Peak FT } \\
\text { at end }\end{array}$ \\
\hline Dominant parameter & Gas gap & Gas gap & Fuel conductivity & Gas gap & Fuel heat rate \\
\hline Dominant sensitivity & $0.38-0.39$ & $0.28-0.20$ & $-0.08--0.15$ & & \\
\hline $\begin{array}{l}\text { Dominant uncertainty, } \\
\%\end{array}$ & $9.1-13.6$ & $9.1-13.6$ & 20 & & \\
\hline Overall uncertainty, \% & $3.4-5.4$ & $2.7-4.1$ & $2.8-4.7$ & 3.8 & 4.4 \\
\hline Overall uncertainty, ${ }^{\circ} \mathrm{C}$ & $15-38$ & $16-50$ & $19-60$ & 38 & 50 \\
\hline
\end{tabular}

Since TC1 and TC2 in Capsule 2 are both located in peripheral locations of the graphite holder, their daily relative temperature standard deviations are the same as shown in Figure 60. Capsule 2 model temperature uncertainty results are presented as follows:

1. The daily standard deviations of five significant input parameters for Capsule 2 are presented in Figure 58.

2. The daily input sensitivities of all AGR-1 time steps for temperatures in Capsule 2 are plotted in Figure 59.

3. The daily relative and absolute standard deviations of TC and fuel compact temperature model uncertainty are presented in Figure 60 and Figure 61.

4. The daily temperature and one standard deviation of the predicted temperatures in Capsule 2 are plotted for: central TC1 and peripheral TC2 in Figure 62, daily average VA FT and daily average peak FT in Figure 63, and TA VA and TA peak FT in Figure 64. 


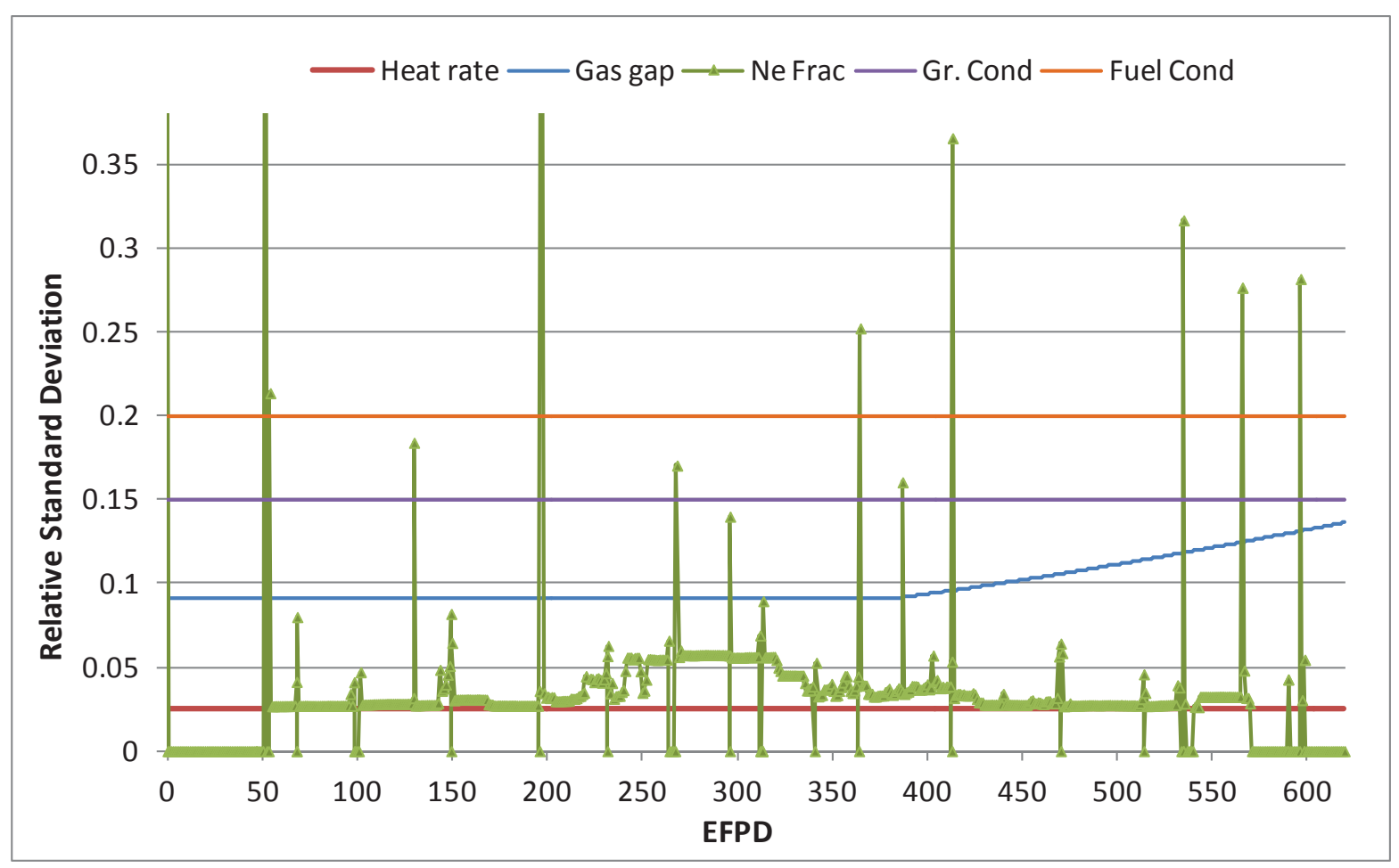

Figure 58. Daily input relative standard deviations for Capsule 2.
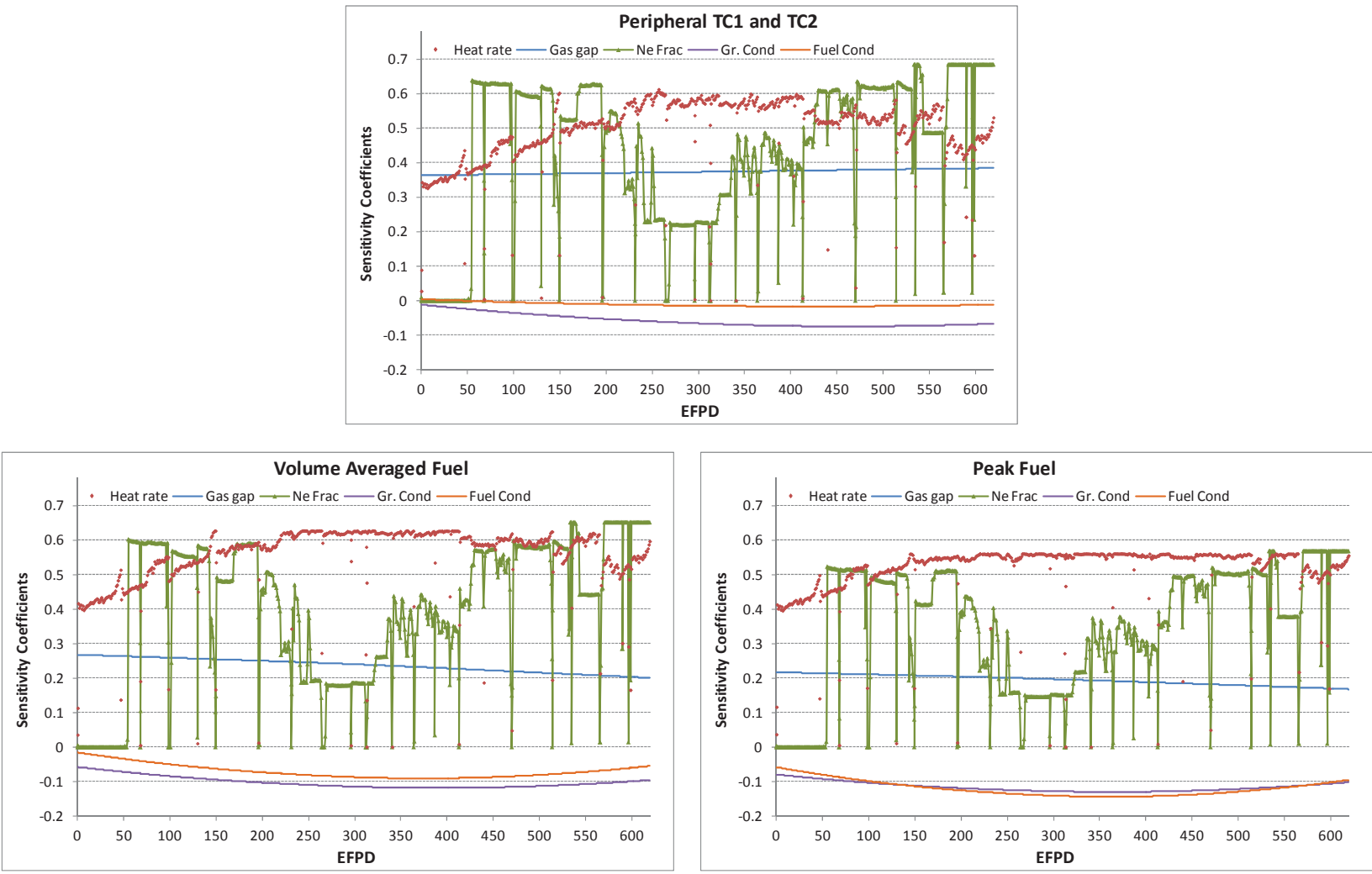

Figure 59. Daily input parameter sensitivities for temperatures in Capsule 2. 


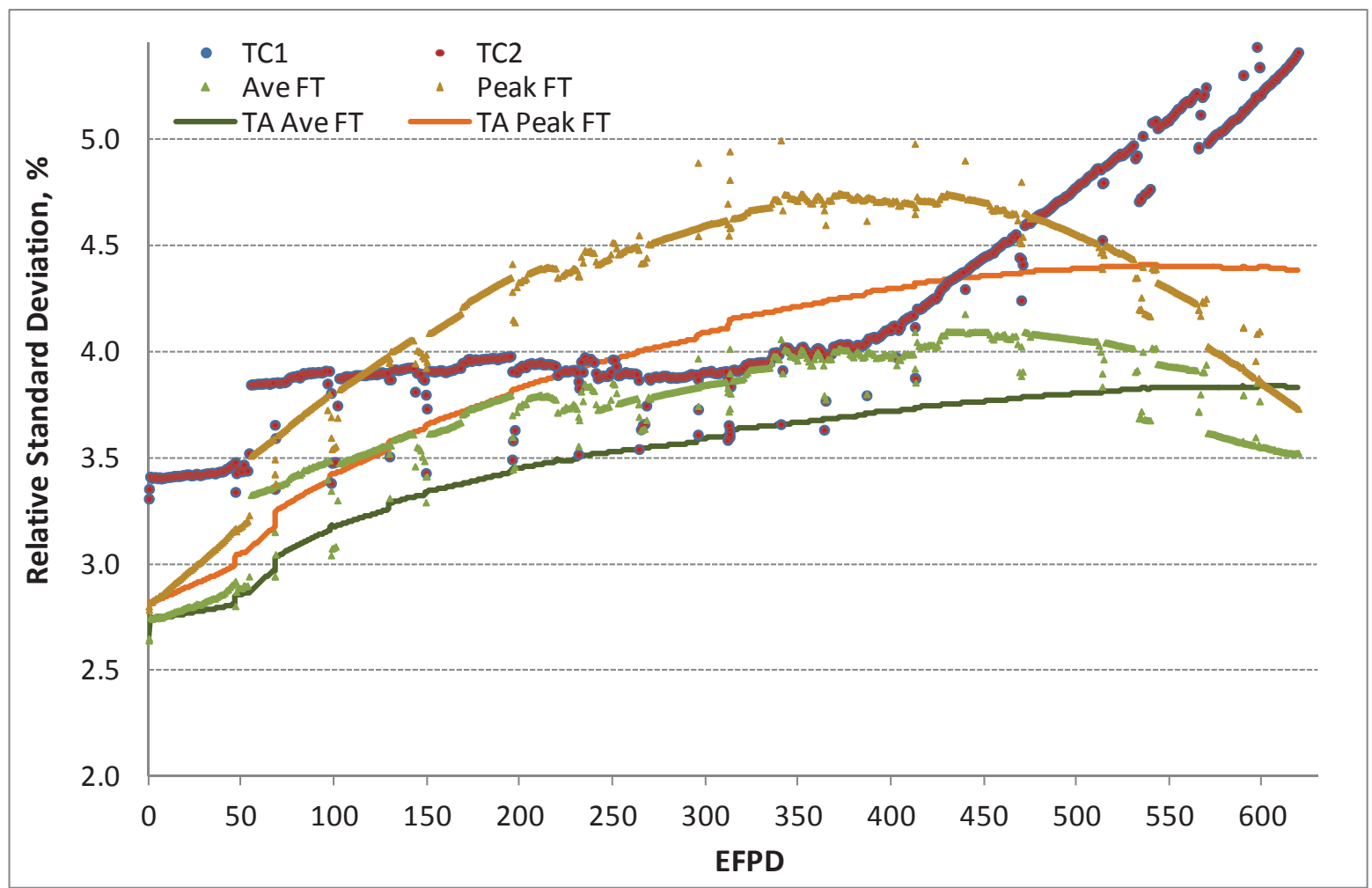

Figure 60. Daily relative standard deviations of predicted TC and fuel temperatures in Capsule 2.

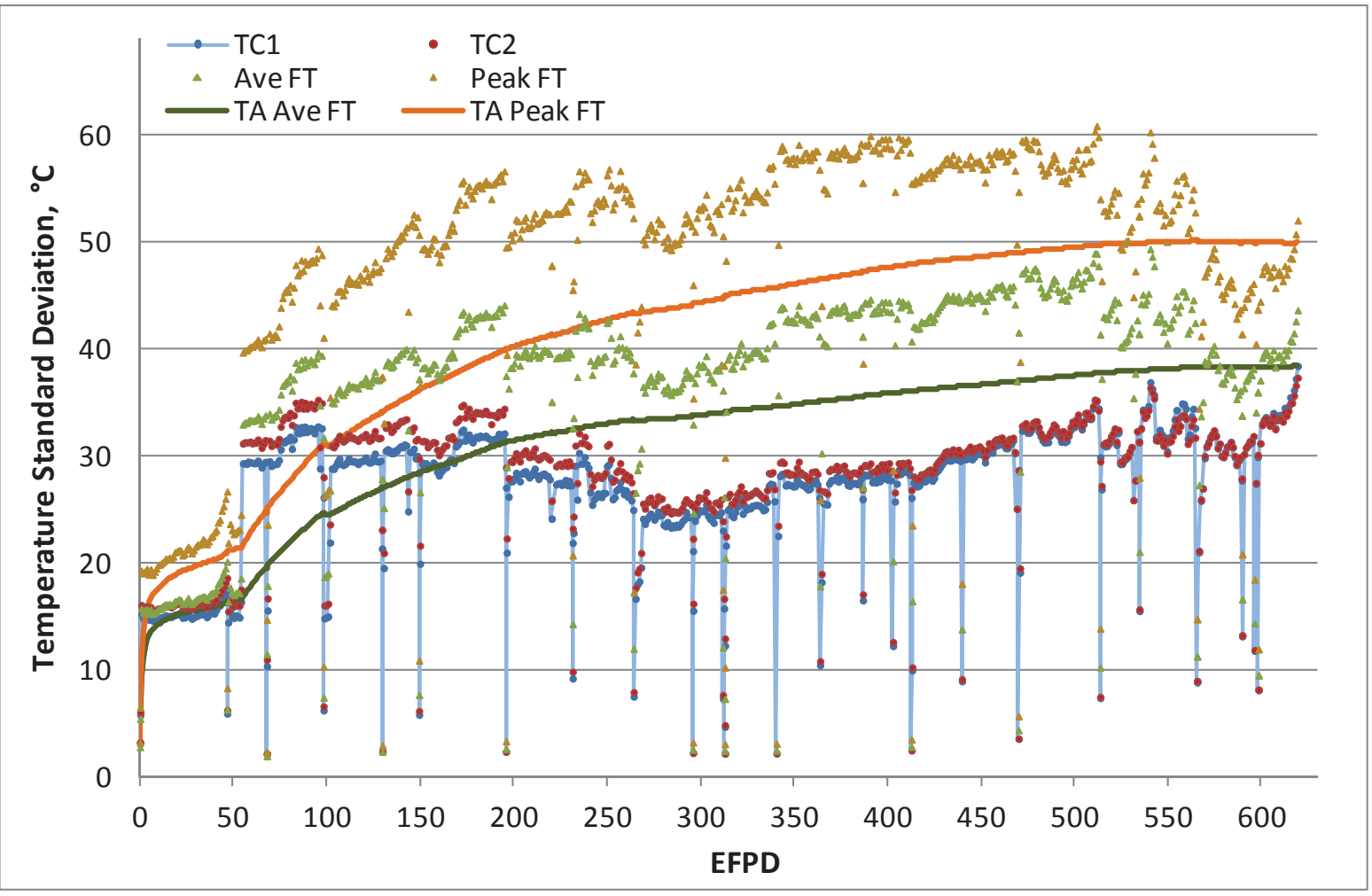

Figure 61. Daily standard deviations of predicted TC and fuel temperatures in Capsule 2. 


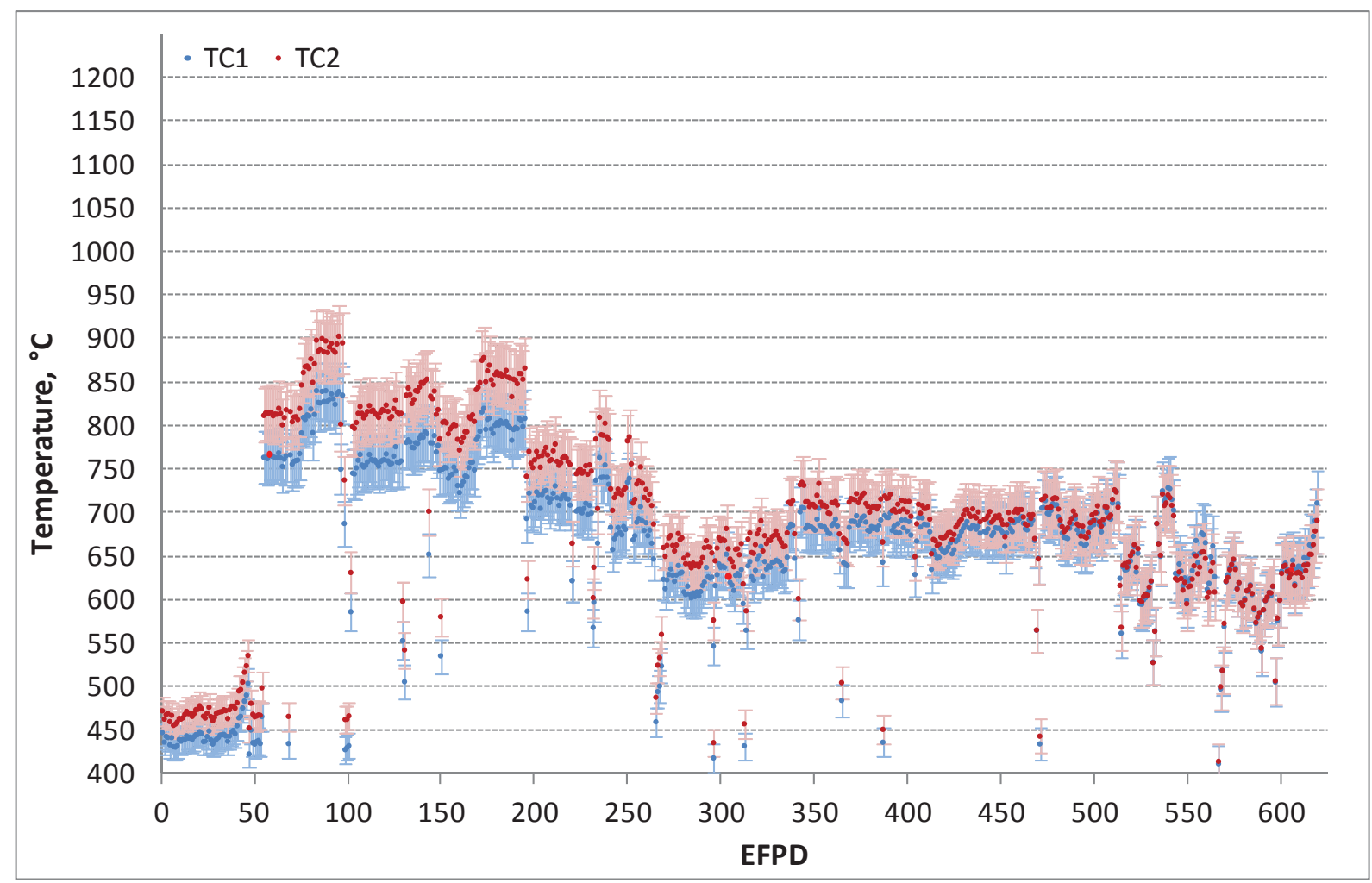

Figure 62. Model temperature and standard deviation of TCs in Capsule 2.

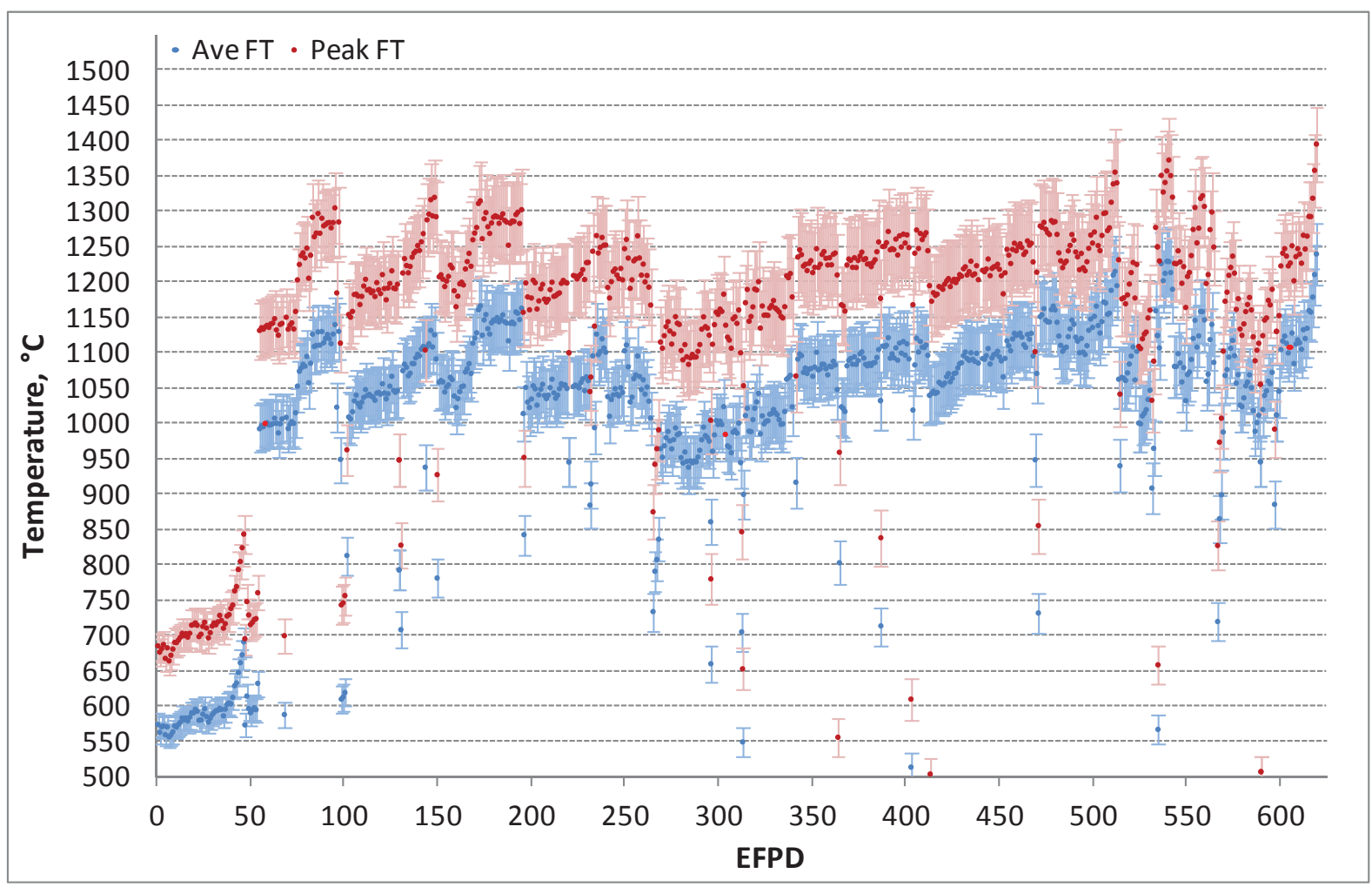

Figure 63. Model temperature and standard deviation of daily fuel temperatures in Capsule 2. 


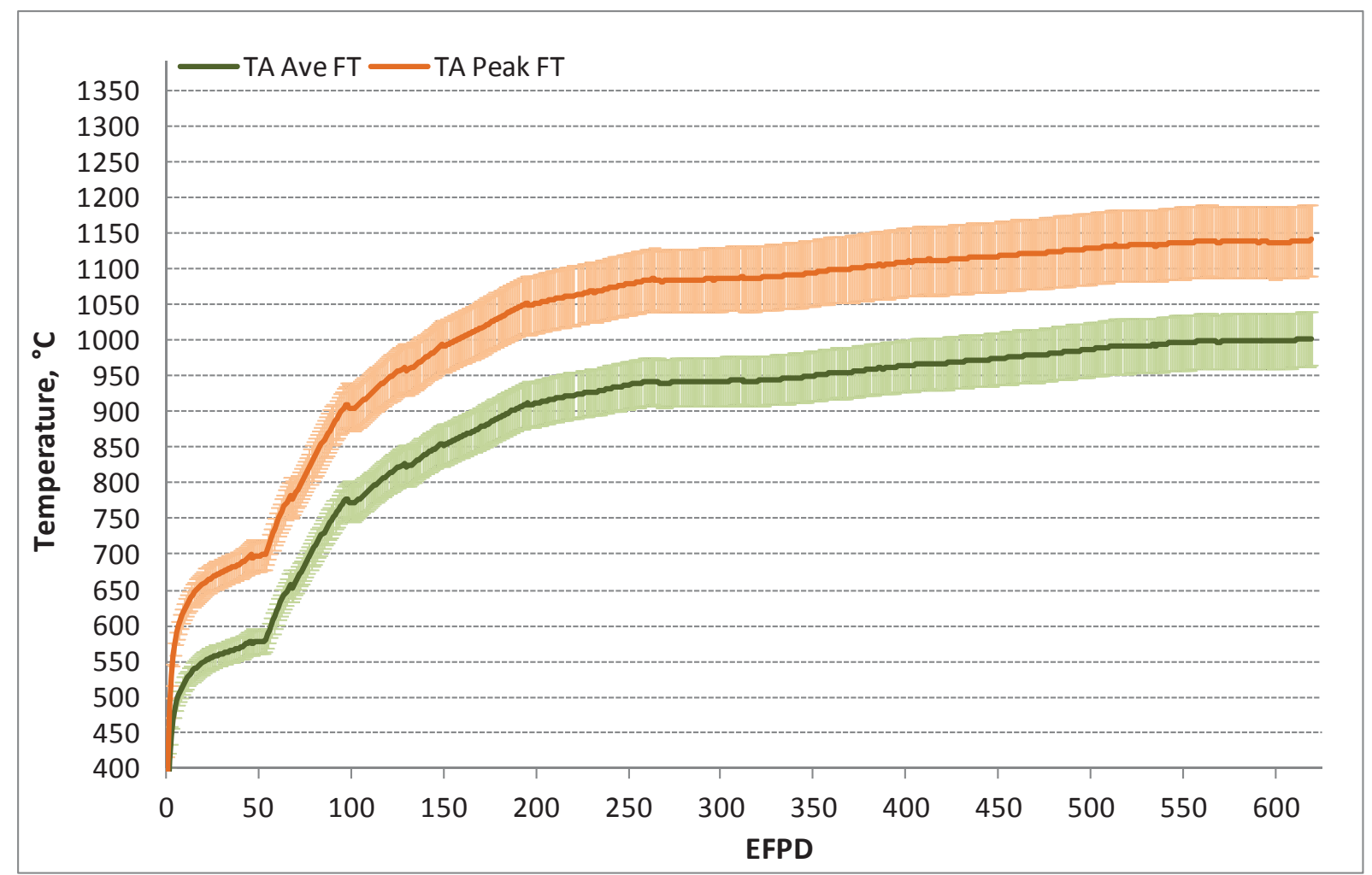

Figure 64. Model temperature and standard deviation of time-average fuel temperatures in Capsule 2.

\subsection{Temperature Uncertainty Results for Capsule 1}

Capsule 1 daily uncertainties (in terms of standard deviations at each time step) are estimated for six calculated temperatures: peripheral TC1, peripheral TC3, daily average VA FT, daily average peak FT, TA VA FT, and TA peak FT. Table 13 summarizes the results for Capsule 1.

Table 13. Summary of temperature uncertainty results for Capsule 1

\begin{tabular}{|l|c|c|c|c|c|}
\hline & $\begin{array}{c}\text { Peripheral } \\
\text { TC1/TC2 }\end{array}$ & VA FT & Peak FT & $\begin{array}{c}\text { TA VA FT } \\
\text { at end }\end{array}$ & $\begin{array}{c}\text { TA Peak FT } \\
\text { at end }\end{array}$ \\
\hline Dominant parameter & Gas gap & Gas gap & Fuel conductivity & Gas gap & Fuel heat rate \\
\hline Dominant sensitivity & $0.32-0.30$ & $0.31-0.30$ & $-0.06--0.16$ & & \\
Dominant uncertainty, $\%$ & $5.5-8.3$ & $5.5-8.3$ & 20 & & \\
Overall uncertainty, $\%$ & $2.0-3.2$ & $2.0-3.7$ & $2.2-4.2$ & 3.2 & 3.8 \\
Overall uncertainty, ${ }^{\circ} \mathrm{C}$ & $10-31$ & $12-45$ & $15-59$ & 33 & 45 \\
\hline
\end{tabular}

Since both TC1 and TC2 in Capsule 1 are located in similar positions in periphery of graphite holder, the model temperatures and standards deviations of these two TCs are similar as shown by the overlapping of their plots in Figure 67, Figure 68, and Figure 69. Capsule 1 model temperature uncertainty results are presented as follows:

1. The daily standard deviations of five significant input parameters for Capsule 1 are presented in Figure 65. Since Capsule 1 had the second largest gas gap distance among the six AGR-1 capsules, the relative gap distance uncertainty for Capsule 1 is smaller than for the four middle capsules. 
2. The daily input sensitivities of all AGR-1 time steps for temperatures in Capsule 1 are plotted in Figure 66. The plots are different for daily peak fuel temperature and temperatures at TC locations. Because of lower boron carbide concentration of $5.5 \%$ in Capsule 1, the sensitivity of graphite thermal conductivity is estimated in a manner similar to that used for Capsule 6, which also has a boron carbide concentration of $5.5 \%$.

3. The daily relative and absolute standard deviations of TC and fuel compact temperature model uncertainty are presented in Figure 67 and Figure 68.

4. The daily temperature and one standard deviation of the predicted temperatures in Capsule 1 are plotted for: peripheral TC1 and TC2 in Figure 69, daily average VA FT and daily peak FT in Figure 70, and TA VA FT and TA peak FT in Figure 71.

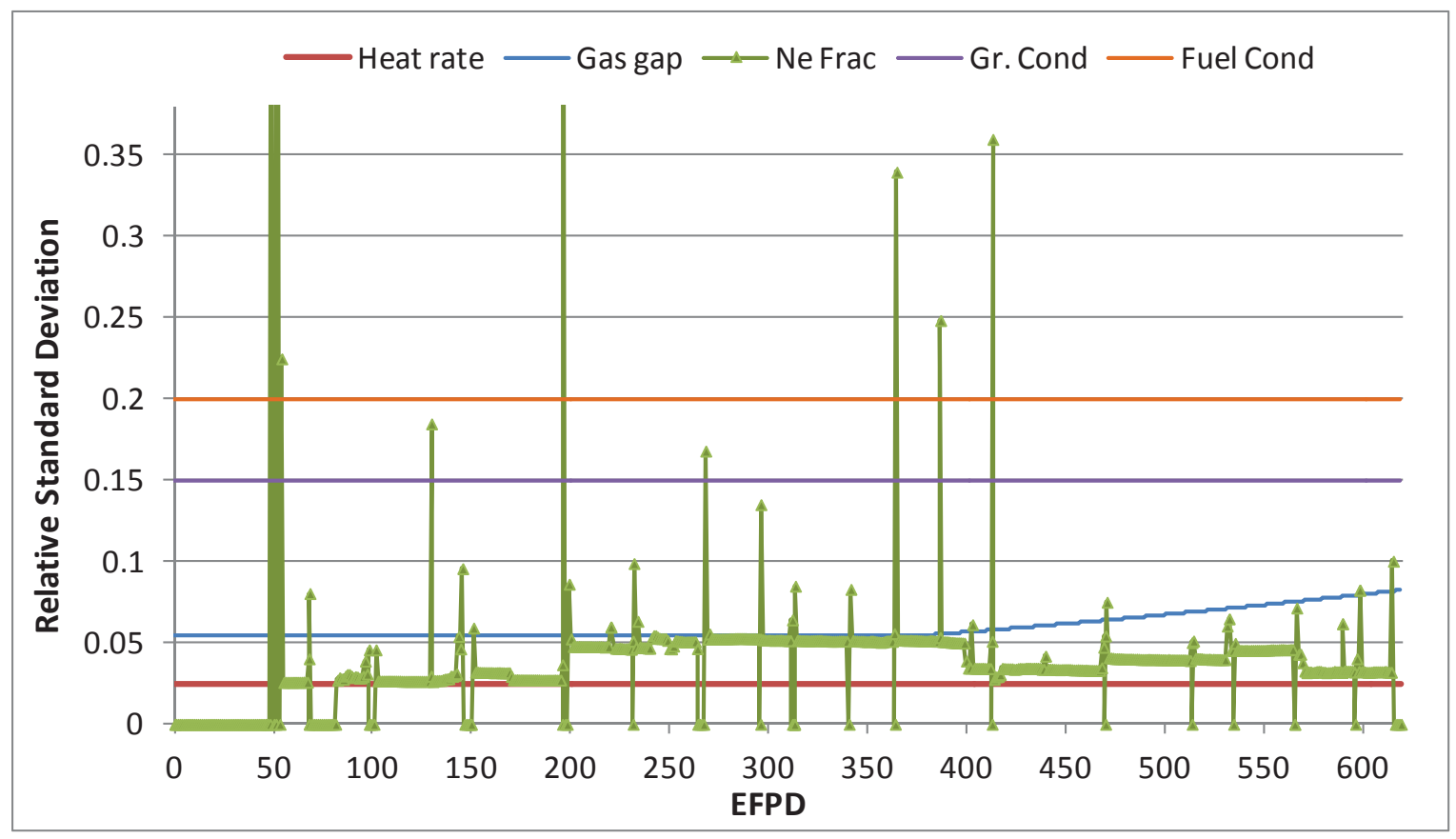

Figure 65. Daily input relative standard deviations for Capsule 1. 

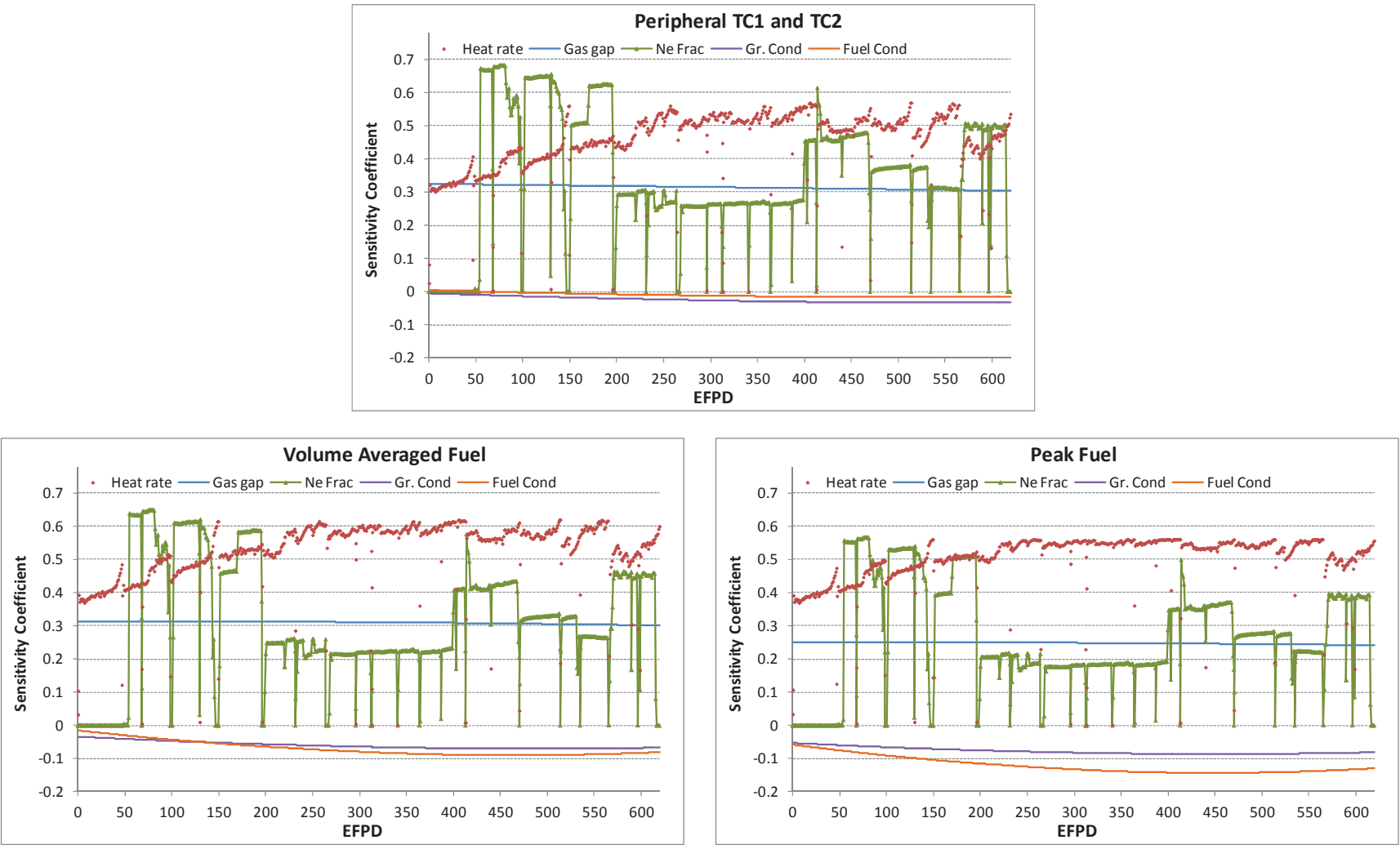

Figure 66. Daily input parameter sensitivities for temperatures in Capsule 1.

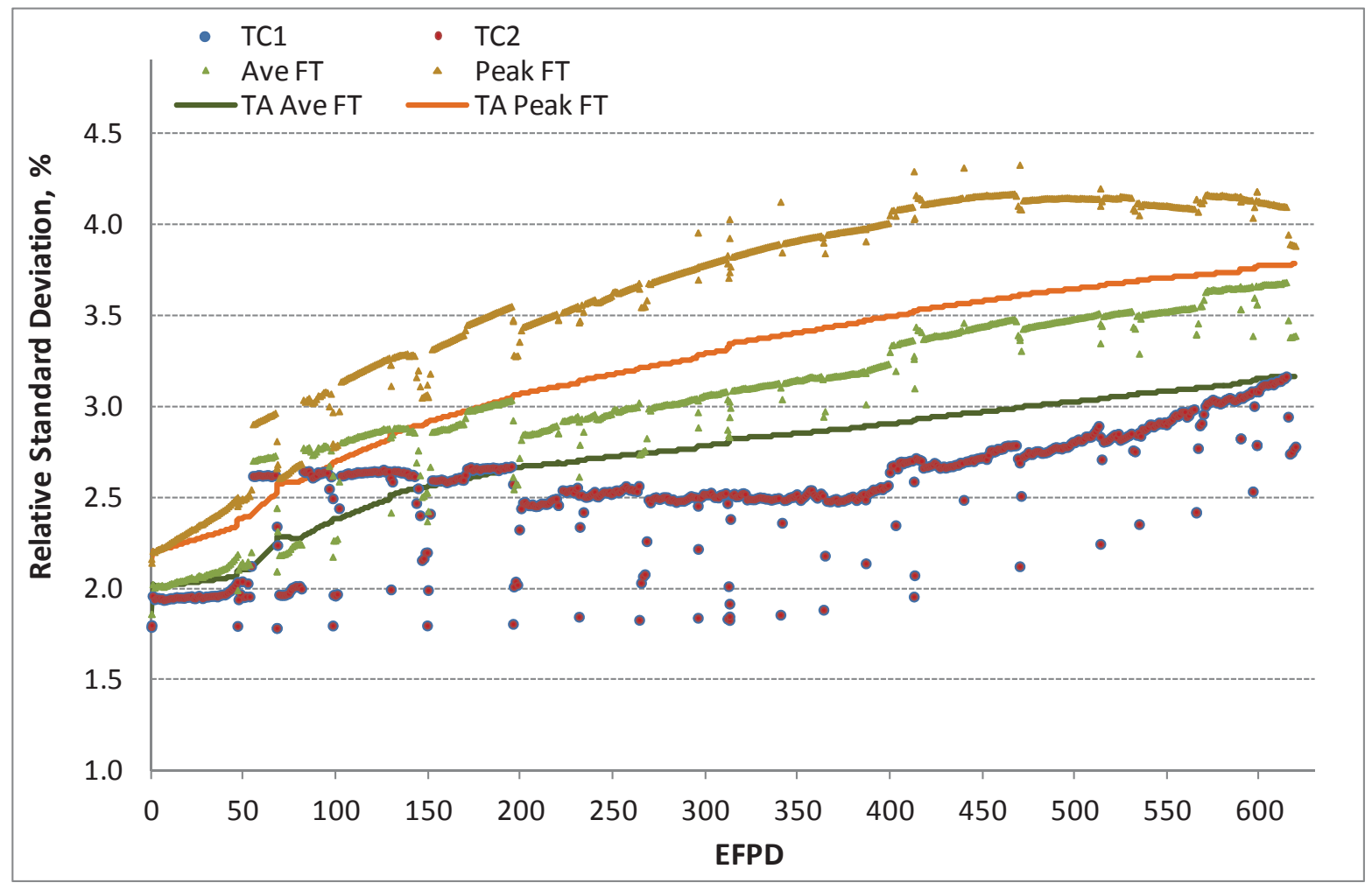

Figure 67. Daily relative standard deviations of predicted TC and fuel temperatures in Capsule 1. 


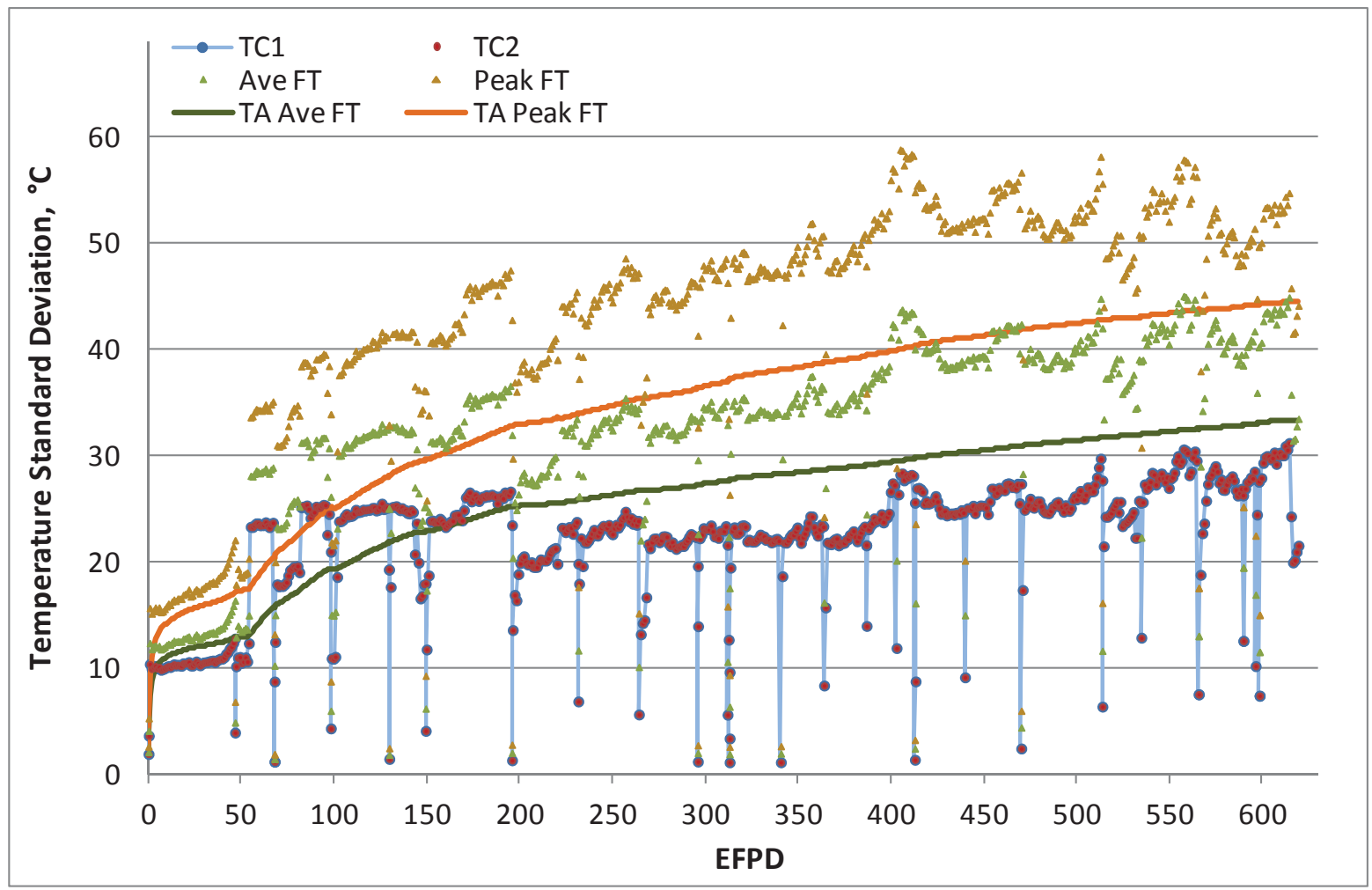

Figure 68. Daily standard deviations of predicted TC and fuel temperatures in Capsule 1.

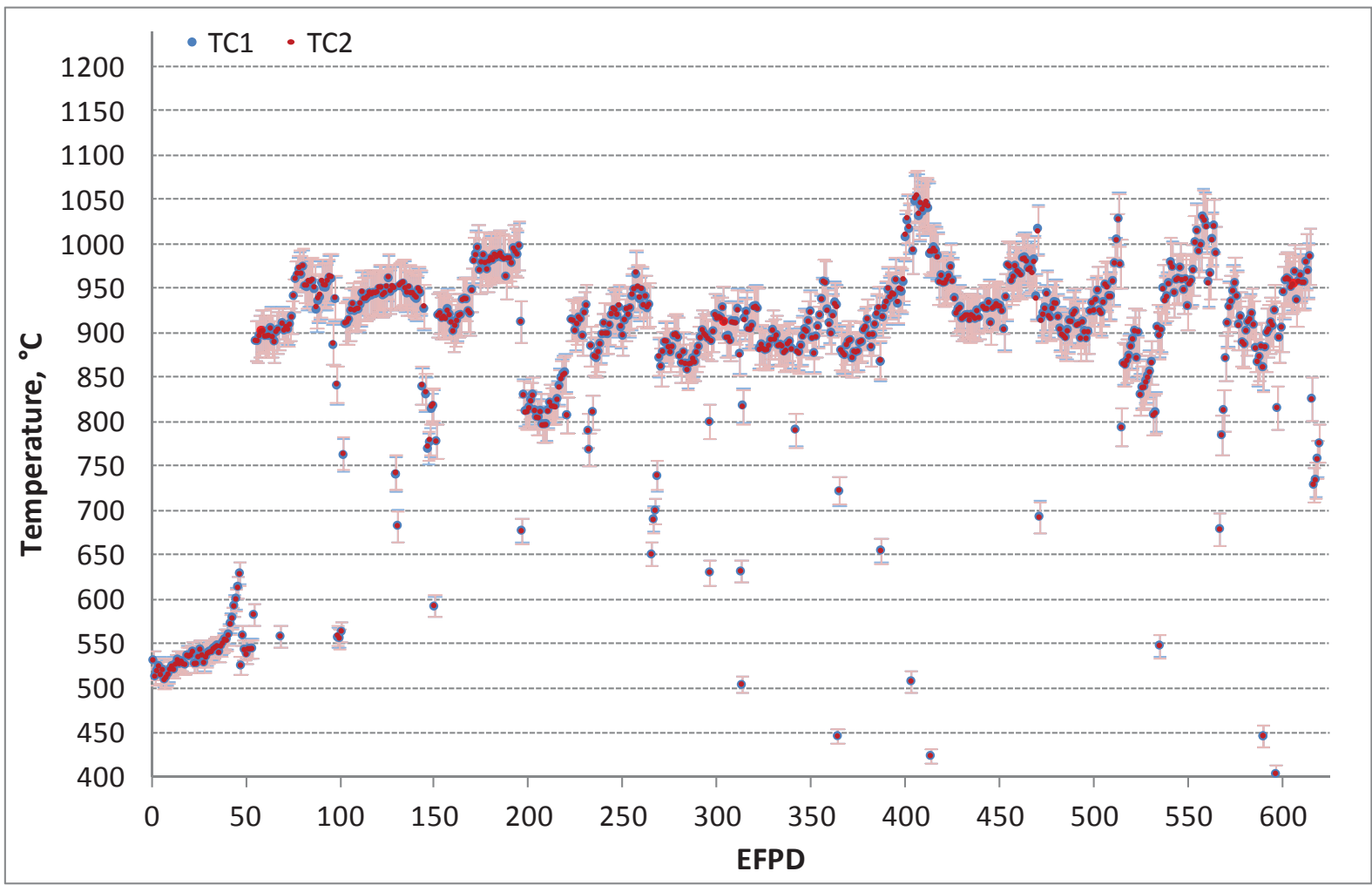

Figure 69. Model temperature and standard deviation of TCs in Capsule 1. 


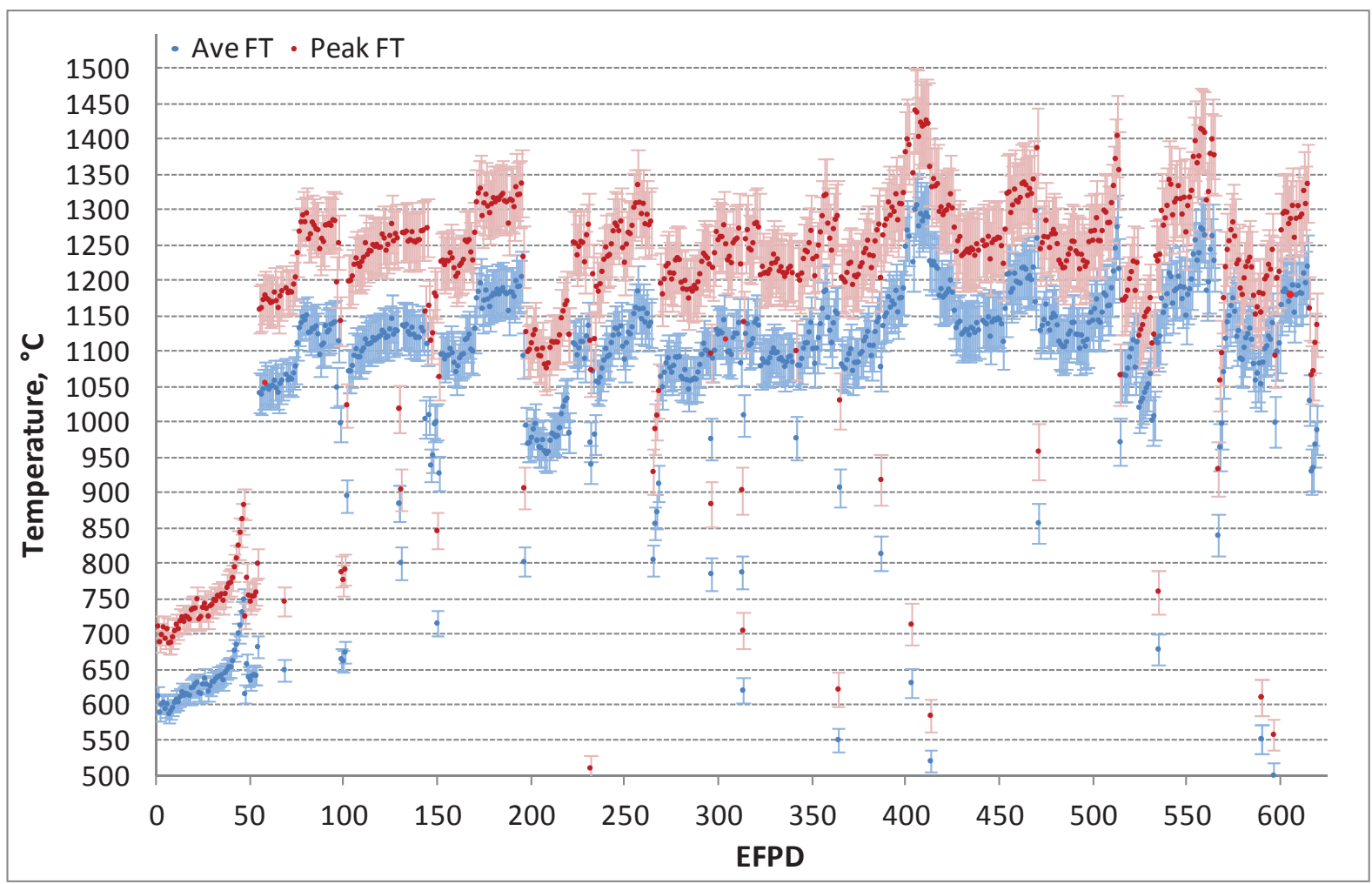

Figure 70. Model temperature and standard deviation of daily fuel temperatures in Capsule 1.

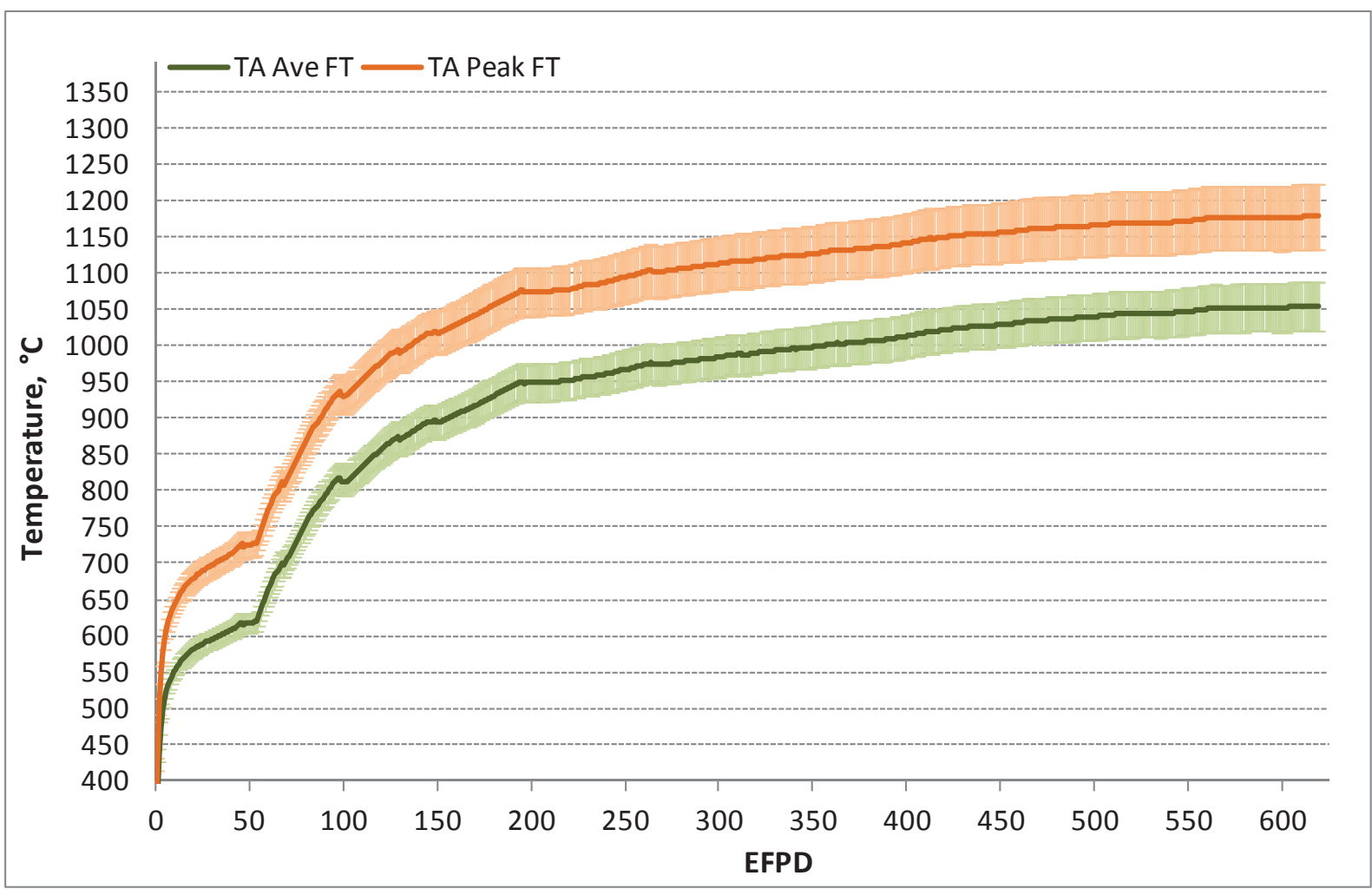

Figure 71. Model temperature and standard deviation of time-average fuel temperatures in Capsule 1. 


\section{CONCLUSIONS}

Knowledge of the thermal conditions and associated uncertainties of the nuclear fuel in a reactor test are central to the interpretation of the test results, and is necessary when using the test results for calibration and validation of nuclear fuel performance models and codes, ultimately in support of the design and licensing of the new nuclear fuel. The work documented in this report supports quantification of uncertainty in the computed thermal condition of nuclear fuels in the AGR-1 test, where it is not practical to obtain direct temperature measurements in the fuel compact domain.

The test was instrumented with TCs to provide temperature measurements in the graphite blocks surrounding the fuel compacts. In-depth analysis and qualification of the TC data were performed in previous work using statistical methods (Pham \& Einerson, 2010) (Pham \& Einerson, 2011). These analyses made use of the ABAQUS thermal simulation results. The AGR-1 TC data were also used to assess and calibrate thermal process models in the ABAQUS code (Hawkes G. , 2012). The calibrated code was then used to predict temperatures in the AGR-1 fuel compact.

This study identifies and analyzes ABAQUS model parameters of potential importance to the AGR-1 predicted fuel temperatures. Expert judgments are used as a basis to specify the uncertainty range for a set of select parameters, including those with high sensitivity and those with large uncertainty. Propagation of model parameter uncertainty is then used to quantify the overall uncertainty of AGR-1 calculated temperatures.

The parameter sensitivity analysis of the thermal model for AGR-1 capsules is performed to determine the sensitivity coefficients of the most influential variables. Capsule 4 data is used for the sensitivity analysis and is assumed to be representative of the other capsules. A sensitivity coefficient describes how the model predicted temperature would be influenced by changes in an input parameter. The overall uncertainty of the model output increases as the absolute sensitivity coefficient of an input parameter increases. The following conclusions are drawn about the parameter sensitivity coefficients for AGR-1 temperature predictions:

- The sensitivity coefficients of fuel fission heat rate and neon fraction are highest (up to 0.7) for all predicted temperatures of interest (volume-average fuel, peak fuel, and TC) for all six capsules.

- The sensitivity coefficients of control gas gap ranged from 0.2 to 0.4 for AGR-1 calculated temperatures. These sensitivities reached their highest values (up to 0.4) for temperatures of the peripheral TCs, especially for the middle capsules (Capsules 3 and 4) which have the smallest gas gaps. The gap sensitivity is much lower $(\sim 0.2)$ for fuel temperatures (volume-average and peak) and center TCs.

- The absolute values of the sensitivity coefficients of fuel compact thermal conductivity and graphite holder thermal conductivity ranged from 0.003 to 0.2 . Even though these sensitivity coefficients are lowest among the five inputs parameters, their high uncertainties $(15 \%$ for graphite holder conductivity and $20 \%$ for fuel compact conductivity) make them significant contributors to the overall uncertainty of the predicted temperatures, especially for target fuel temperatures. The conductivity sensitivity coefficients vary for different temperatures as follows: (1) They are near zero for peripheral TC temperatures (or the peripheral TC temperatures are insensitive to variations of fuel conductivity and graphite holder conductivity); and (2) they are much higher for fuel and central TC temperatures. The sensitivity coefficient of fuel conductivity reached the highest absolute value of 0.2 for peak fuel temperature at the middle of the AGR-1 irradiation.

The overall uncertainty of calculated temperatures, in terms of one standard deviation, is obtained through propagation of model parameter uncertainty as the square root of the summation of the parameter 
variances weighted by the squares of their sensitivity coefficients. Thus, the effect of a parameter uncertainty on the model prediction variation is a product of input uncertainty and the sensitivity coefficient. The most significant factors contributing to overall uncertainty of the AGR-1 temperature predictions are:

- For the top capsule, Capsule 6 , the positive $10 \%$ bias in the fuel fission heat rate drives the overall uncertainties (up to $90 \%$ of overall standard deviation) of all predicted temperatures (TC, volumeaverage, and peak).

- For the other capsules, the most influential factor depends on the temperature parameter:

- For volume-average fuel temperature and peripheral TC temperature, the control gas gap is the most influential factor on overall uncertainty, especially at the beginning (due to higher gap sensitivity) and the end (due to higher gap uncertainty) of the AGR-1 test. The gas gap uncertainty influences the overall uncertainty of the peripheral TC temperature more than the volume-average fuel temperature.

- For center TC temperature, the graphite holder thermal conductivity, which has the second largest uncertainty of $15 \%$ and sensitivity coefficients as much as -0.2 , is the most influential factor for most of the time.

- For peak fuel temperature, the most influential factor is the fuel compact thermal conductivity, which has the largest uncertainty $(20 \%)$ and a large sensitivity coefficient (up to $-0.15)$, followed by the gas gap and then the graphite holder thermal conductivity. The influence of fuel compact thermal conductivity was greatest during the middle of the AGR-1 irradiation.

The overall uncertainty in the calculated temperatures for AGR-1 ranged from $2.0 \%$ to $6.5 \%$, depending on irradiation time (thermal conditions), capsule, and the temperature quantity being predicted (peak, volume-average, or TC). Result highlights are:

- For temperatures at TCs, the overall uncertainty ranged from $2 \%$ to $6.5 \%$. The highest relative uncertainty at the end of AGR-1 is $6.5 \%\left(\sim 48^{\circ} \mathrm{C}\right)$ for the peripheral TC in Capsule 3 and $6 \%$ $\left(\sim 45^{\circ} \mathrm{C}\right)$ for the peripheral TCs in Capsules 4 and 6 . This uncertainty of predicted temperatures at the peripheral TCs is caused mainly by the increasing uncertainty of the control gas gap distance, especially for the middle capsules at the end of irradiation. The increase of gap uncertainty has more effect on the temperature uncertainty of peripheral TCs than on the uncertainty of the center TC.

- For fuel temperatures, the volume-average temperature uncertainties are lower than peak temperature uncertainties: (i) the relative uncertainty ranged from 3\% to $4 \%$ for volume average temperatures; and (ii) ranged from $3 \%$ to $5 \%$ for peak temperatures (up to $\sim 65{ }^{\circ} \mathrm{C}$ ). The fuel temperature uncertainty reaches its highest value at the time when the sensitivity coefficients of fuel and graphite thermal conductivity are highest leading to large variation of calculated fuel temperatures.

- The time-average volume-average fuel temperature uncertainty reaches $3.7 \%\left(\sim 40{ }^{\circ} \mathrm{C}\right)$ and the time-average peak fuel temperature uncertainty reaches $4.4 \%\left(\sim 52{ }^{\circ} \mathrm{C}\right)$ after two thirds of the irradiation.

The sensitivity analysis performed in this work went beyond the traditional local sensitivity. Using experimental design, analysis of pairwise interactions of model parameters was performed to establish sufficiency of the first-order (linear) expansion terms in constructing the response surface. To achieve completeness, uncertainty propagation made use of pairwise noise correlations of model parameters. Further, using an interpolation scheme over the input parameter domain, the analysis obtains time- 
dependent sensitivity over the test campaign's duration. This allows computation of uncertainty for the predicted peak fuel temperatures and the predicted graphite temperatures at TC locations over the whole AGR-1 irradiation period.

In addition to model-parameter uncertainties analyzed in this study, other epistemic uncertainties exist. In this case, these uncertainties can be categorized into three groups. The first group belongs to biases and errors in expert assessment of the range of uncertainty associated with input parameters. This includes the parameter range and probability density function (pdf) of the parameter distribution. The second group includes modeling assumptions used to build the ABAQUS model for the AGR-1 test. The third group is associated with numerical treatment (e.g., resulting in discretization errors) needed to implement and operate the ABAQUS simulations. Although the effect of the first and second groups is generally very hard to evaluate, it is important to systematically delineate them, so not to over-state the confidence in predicted values (underestimating their uncertainties) stemming from a model-parameter uncertainty analysis alone.

For the case at hand, the second group (e.g., model-form uncertainties) is judged to be dominant. Specifically, the sensitivity of the thermal simulation results to the gap distance suggests that modeling of phenomena that govern gap thermal resistance is expected to matter the most. It is recommended that future work assess the effect of material swelling/shrinkage and thermo-mechanical deformation (e.g., causing asymmetrical changes of gap distance) and surface emissivity. 


\section{REFERENCES}

Abbott, M., Hull, L., Pham, B., and Plummer, M. (2010). AGR-1 Data Qualification Report. Idaho National Laboratory: INL/EXT-10-17943.

Demkowicz, P., Cole, L., Ploger, S., and Winston, P. (2011). AGR-1 Irradaiated Test Train Preliminary Inspection and Disassembly First Look. INL/EXT-10-20722.

Gontard, R., \& Nabielek, H. (1990). Performance Evaluation of Modern HTR TRISO Fuels. Forschungszentrum Julich GmbH. HTA-IB-05/90.

Hawkes G.L., e. a. (2011). Sensitivity Evaluation of the Daily Thermal Predictions of the AGR-1 Experiment in the Advanced Test Reactor. Nice, France: ICAPP 2011 Conference.

Hawkes, G. (2012). AGR-1Daily As-run Thermal Analyses. Idaho National Laboratory: ECAR968, Rev. 3.

Hawkes, G. (2011b). Uncertainty Analysis ABAQUS Runs Email.

Maki, J. (2009). AGR-1 Irradiation Experiment Test Plan. Idaho National Laboratoty: INL/EXT05-00593, Rev. 3.

Ostle, B., and Mensing, R. (1975). Statistics in Research (3rd Edition ed.). Ames, Iowa: Iowa State University Press.

Petti, D. (2010). Technical Program Plan for the Next Generation Nuclear Plant/Advanced Gas Reactor Fuel Development and Qualification Program. Idaho National Laboratory: PNL-3636, Rev. 2.

Petti, D., Maki, J., Ambrosek, R., Hawkes, G., Einerson, J., et al. (2011). NGNP Program Metting for AGR-1 Thermal Model Input Uncertainty Estimation. INL.

Pham, B., and Einerson, J. (2011). Simulation-Aided Qualification of Thermocouple Data for AGR Experiments. Trans. Am. Nucl. Soc., 104. Hollywood, FL.

Pham, B., and Einerson, J. (2010). Statistical Analysis Support for Nuclear Fuel Performance Experimental Data Qualification. San Diego: ANS Conference Proceeding.

Pham, B., Hawkes, G., and Einerson, J. (2012). Improving Thermal Model Prediction through Statistical Analysis of Irradiation and Post-Irradiation Data from AGR Experiments. HTR 20123-010. Tokyo, Japan: October 28 - November 1, 2012.

SAS. (2009). JMP 8 Statistics and Graphics Guide. Cary, NC: SAS Institute Inc., Second Edition.

Snead, L., and Burchell, T. (1995). Reduction in Thermal Conductivity Due to Neutron Irradiation. 22nd Biennial Conference on Carbon, (pp. 774-775).

Sterbentz J.W., e. a. (2011). Monte Carlo Depletion Calculation for the AGR-1 TRISO Particle Irradiation Test. San Diego: ANS Annual Conference.

Sterbentz, J. W. (2011b). JMOCUP As-run Daily Physics Depletion Calculations for the AGR-1 Test in the ATR B-10 Position. Idaho National Laboratory: ECAR-958, Rev. 1. 